\title{
Exploiting Task-Doc ument Relations in Support of Information Retrieval in the Workplace
}

\author{
Luanne Silvia Freund \\ A thesis submitted in conformity with the requirements \\ for the degree of Doctor of Philosophy \\ Faculty of Information Studies \\ University of Toronto
}

(C) Copyright by Luanne Silvia Freund 2008 


\title{
Exploiting Task-Document Relations in Support of Information Retrieval in the Workplace
}

\author{
Luanne Silvia Freund \\ Doctor of Philosophy \\ Graduate Faculty of Information Studies \\ University of Toronto
}

2008

\begin{abstract}
Increasingly, workplace information seeking takes place in digital information environments and is reliant upon search systems. Existing systems are designed to retrieve information that is relevant to the query, but are not capable of identifying information that is well-suited to the context and situation of a search. This is a problem for professionals who often are searching for a small amount of useful information that can be applied to a problem or task, and have limited time to browse through large sets of results. This inability of search systems to discriminate between relevant and useful documents is one of the core problems in information retrieval.
\end{abstract}

In this dissertation, I address this problem by studying the role that contextual factors play in determining how a group of professionals searches for and selects information. The central question concerns the nature of the relationships between these contextual factors, specifically between the genres in the document collection and the tasks of the searcher, with an aim to exploit such relationships to improve workplace information retrieval. Research was conducted through multiple studies in three phases, moving from an exploratory study of workplace information behaviour to a controlled experimental user study.

Findings confirm that workplace context shapes search behaviour. This relationship is modeled as a set of key contextual factors and sets of context-dependent access constraints, preferred document characteristics, and search strategies. Among the contextual factors identified, work 
tasks and information tasks were found to be significantly associated with document genres. This task-genre relationship was modeled as a matrix of associations between domain-specific task and genre taxonomies and successfully implemented as a filtering component in a workplace search system. This is the first major study of the relationship between task and genre in information seeking and of its application to information retrieval systems. 


\section{Acknowledgements}

This document has been a long time coming and many people have helped me along the path to its completion. First among these are my wonderful children, Alon, Jonathan, Gabriel and Ethan, who, despite the injustice of having to grow up with a mother who never seemed to be able to finish her homework, have consistently cheered me on and always provided hugs when needed. A great debt of thanks is also owed to my partner of many years, Amir, who encouraged me to take on this project, and who has supported our family through my many years as a student.

I have been extremely fortunate to work with Elaine Toms throughout my doctoral studies. Elaine is a fine supervisor and mentor and a good friend, who guided me through this research, introduced me to academia, and with whom I have travelled to many beautiful places, shared many glasses of wine, and had a great many heated and enjoyable discussions about research in particular and life in general. What an adventure! It has been a privilege to work with the other members of my committee as well. Brian Cantwell Smith and Chun Wei Choo provided wise counsel when needed and Mark Chignell got the whole project underway and helped to guide it right through to the finish line.

This work is the culmination of a research project funded over a number of years by the IBM Centre for Advanced Studies (CAS) in the Toronto Software Lab. This was a wonderful opportunity for many reasons, not least of which was the chance to work with great people. Kelly Lyons, Paul Smith, Gordon Lee, Elena Litani and other members of the CAS staff helped to support the research and provided valuable input at different stages; IBMers David Modjeska, Jack Boyce, Dory Mazina, Mike Capern, Bruce Buscher and many others helped to shape and promote the project; and Julie Waterhouse was a daily tonic of organization, optimism, friendship and good sense over several years. While at CAS, I had the opportunity to collaborate with other students and researchers. Chief among these is Charlie Clarke, from whom I learned a great deal 
about IR and who, together with Peter Yeung, made the implementation of the X-Site system possible.

Colleagues at the Faculty of Information Studies provided yet another network of support and guidance over the course of this work. I dearly wish that Ethel Auster was still with us to celebrate the completion of this dissertation, as she was a stalwart source of encouragement and advice in the early stages. Many others at FIS have helped and supported my work as well, among them Joan Cherry and Wendy Duff, and fellow students: Joan, Mary, Greta, Scott, Jean, Jane, Lisa, Herman, and Krista. New colleagues at UBC have continued to provide assistance when needed: Rick Kopak, a ready source of advice and encouragement, and Christina Nilsen, who helped to edit and format this document.

This is a long list and could certainly be much longer, so to all of those whom I mentioned and did not mention - thank you. And finally, to my wonderful mother, brothers and sisters, thanks for sticking with me along this long and winding path. 


\section{Table of Contents}

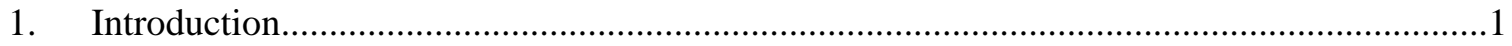

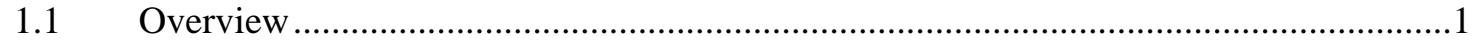

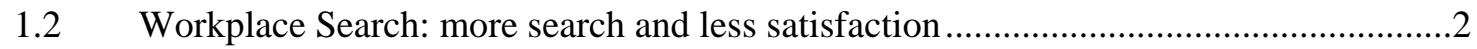

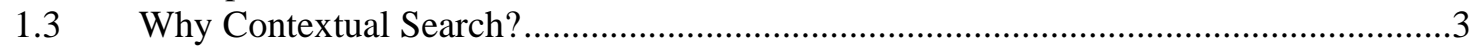

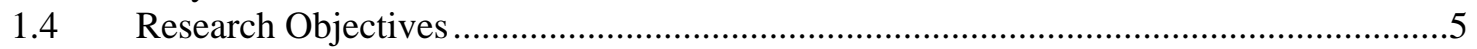

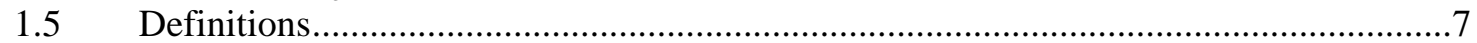

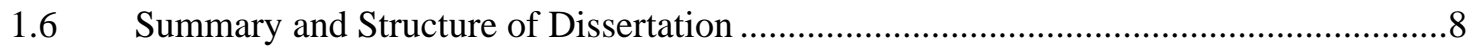

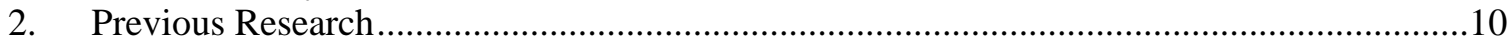

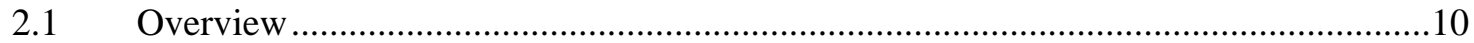

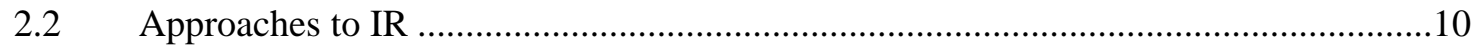

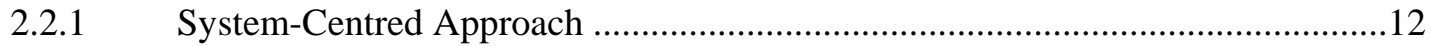

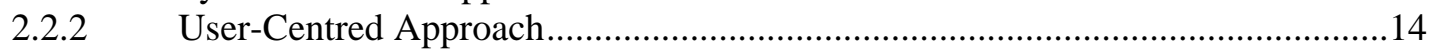

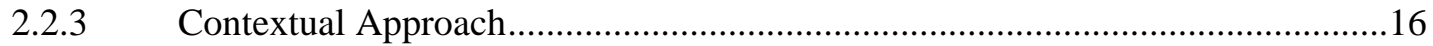

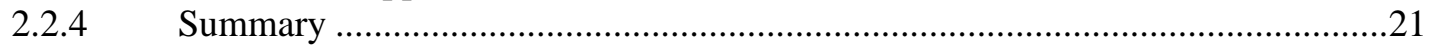

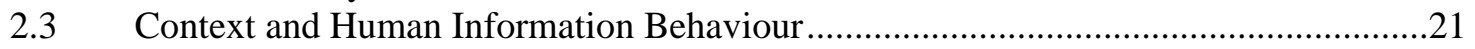

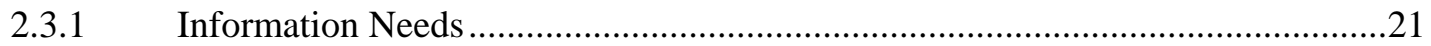

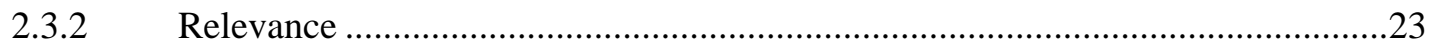

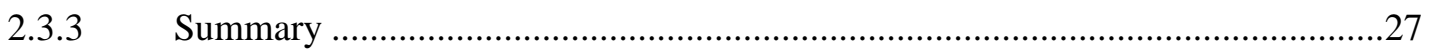

2.4 Workplace Information Seeking and Retrieval ......................................................28

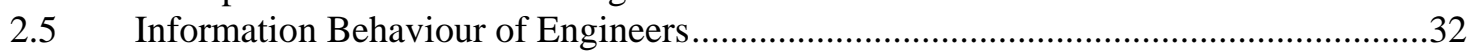

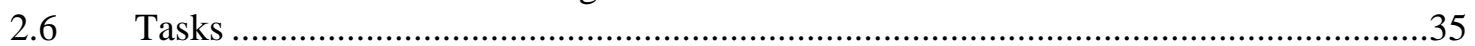

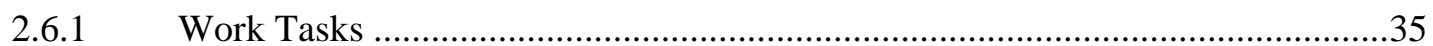

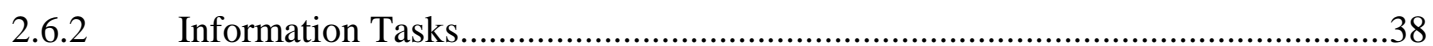

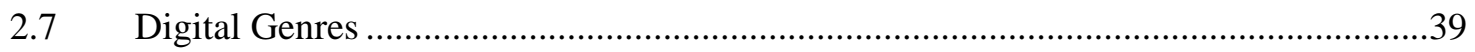

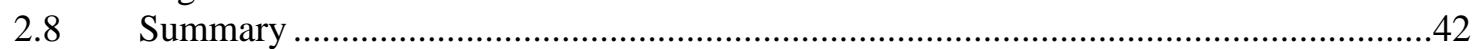

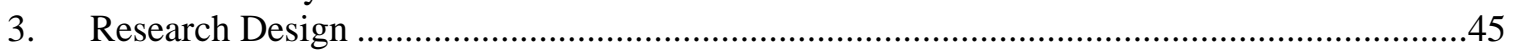

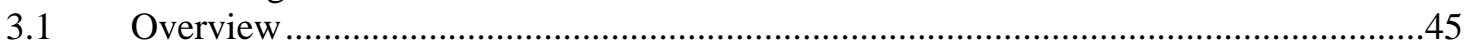

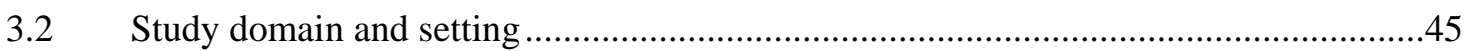

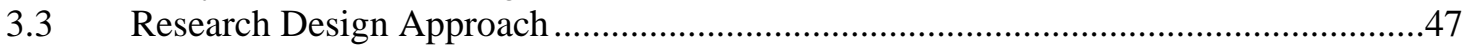

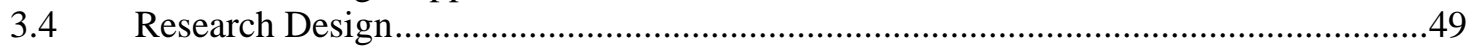

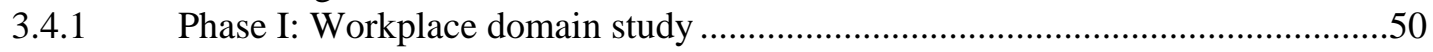

3.4.2 Exploration and Validation of Task-Genre Relationships...................................52

3.4.3 Implementation and Evaluation of Task-Genre Relationships ............................53

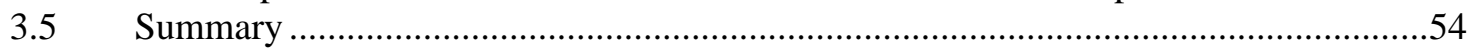

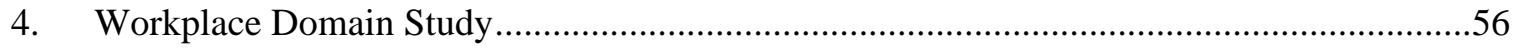

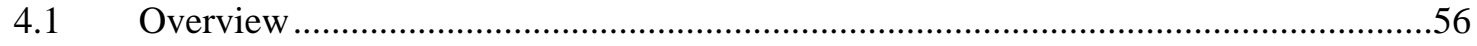

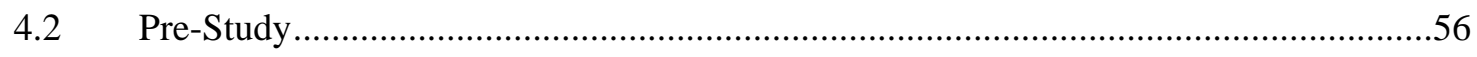

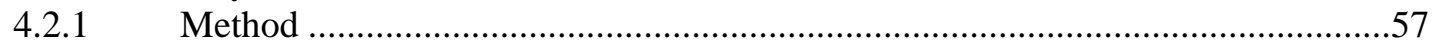

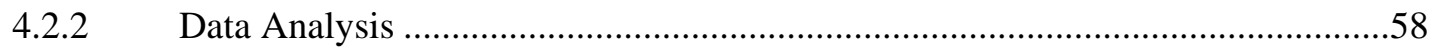

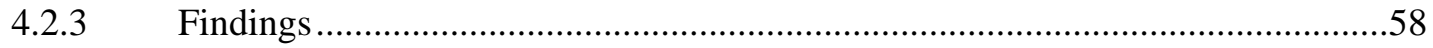

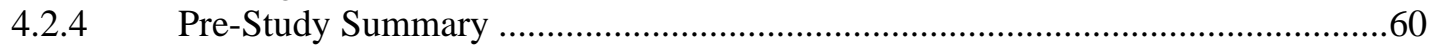

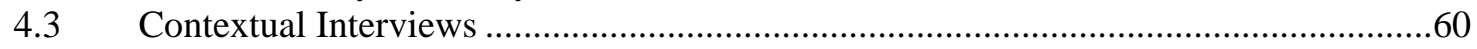

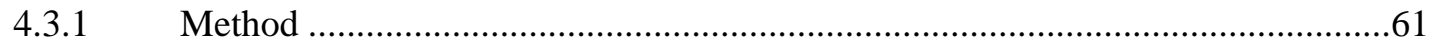

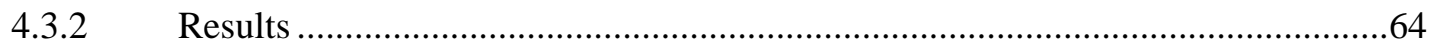

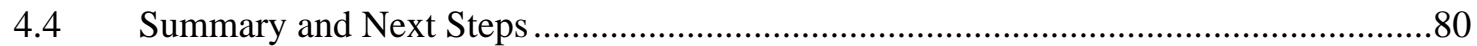

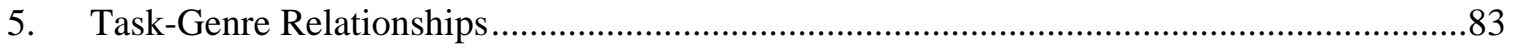

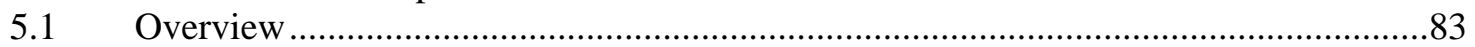

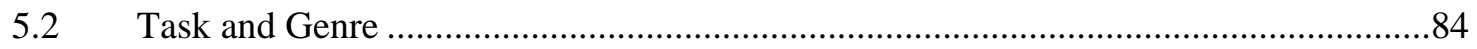




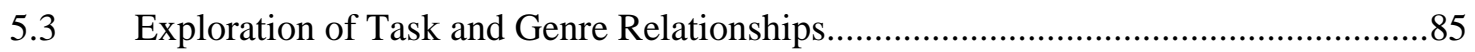

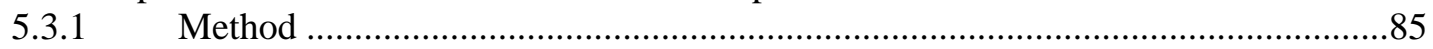

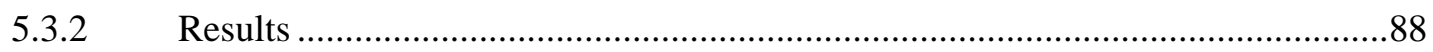

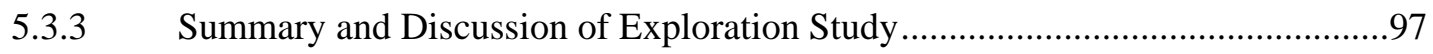

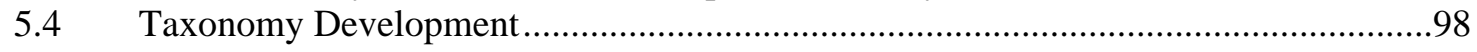

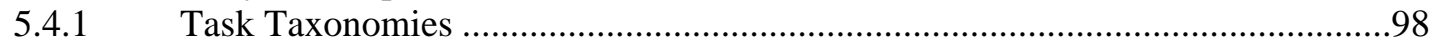

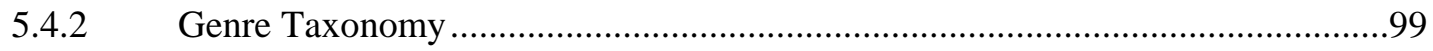

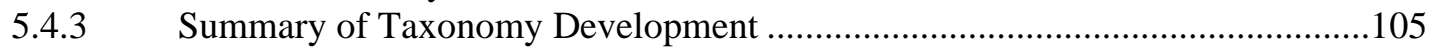

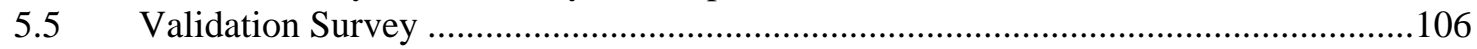

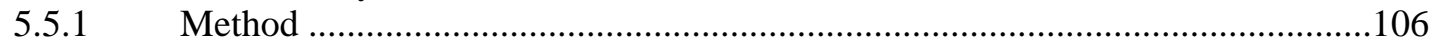

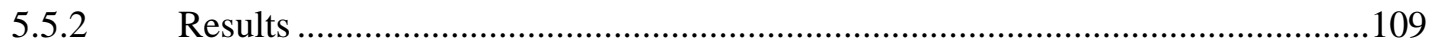

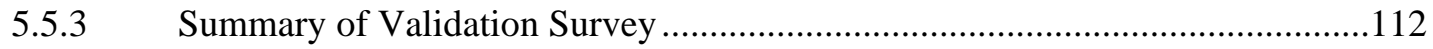

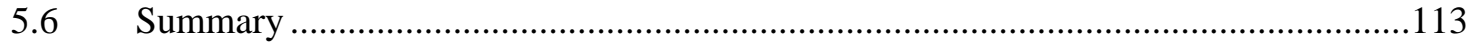

6. Implementing Task-Genre Relationships in Search ......................................................114

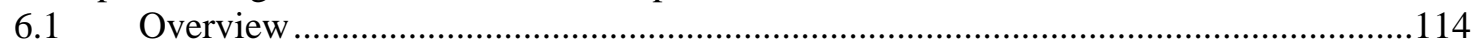

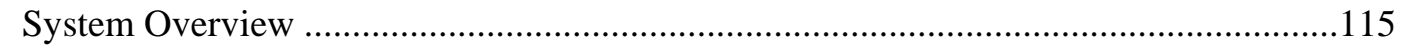

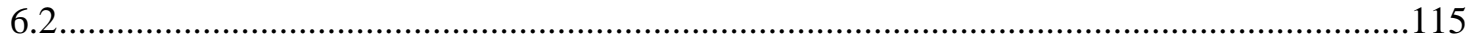

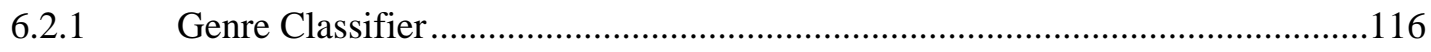

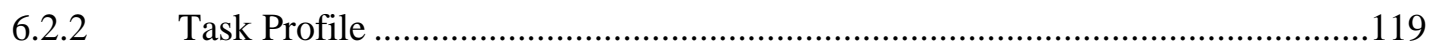

6.2.3 Task-Genre Associations Matrix (TGAM) ................................................120

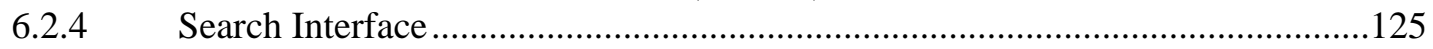

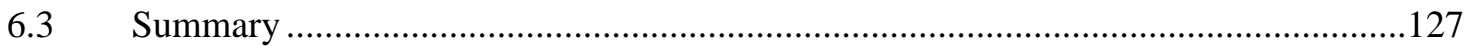

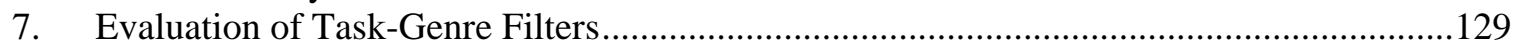

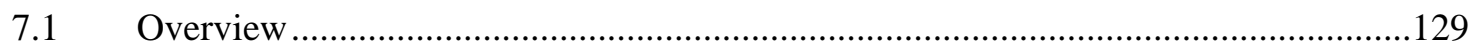

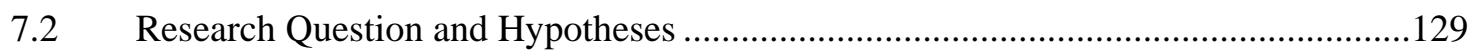

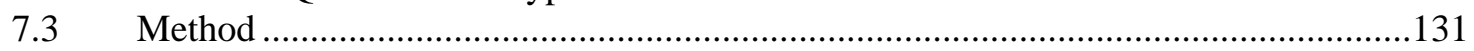

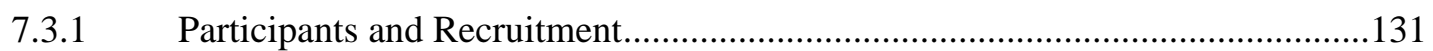

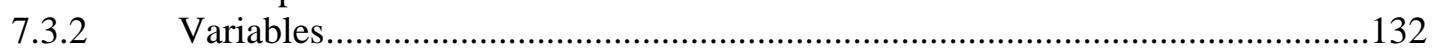

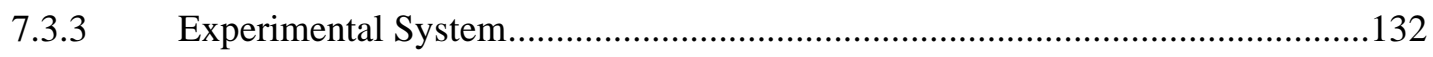

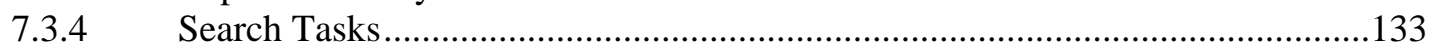

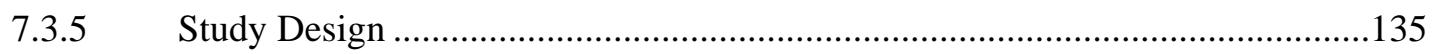

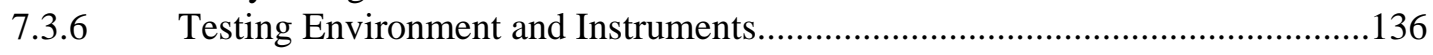

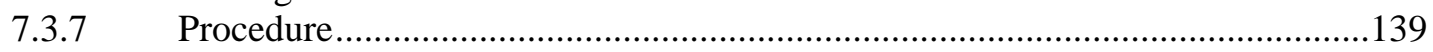

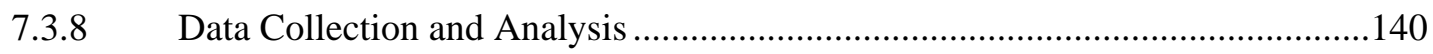

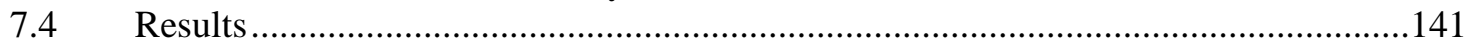

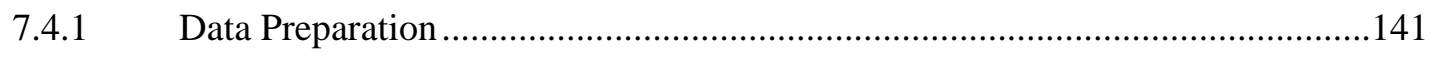

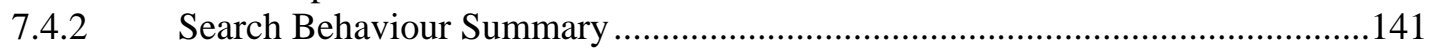

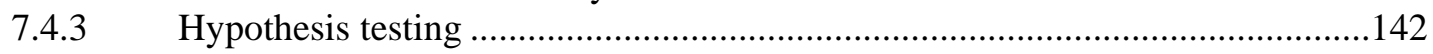

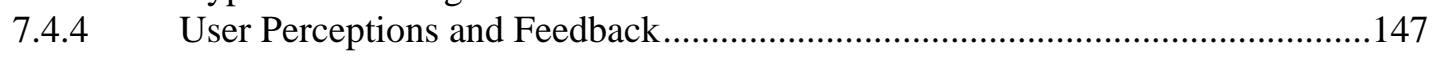

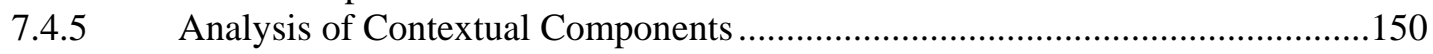

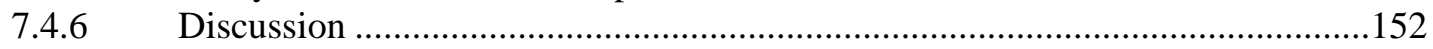

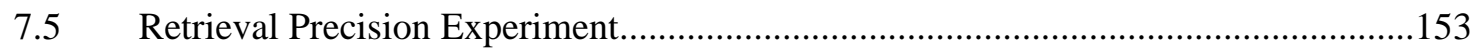

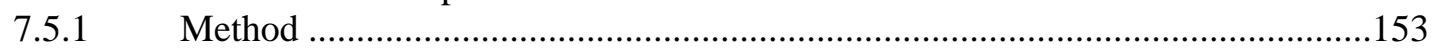

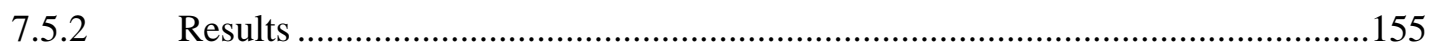

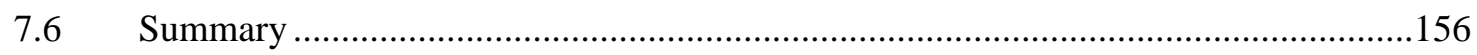

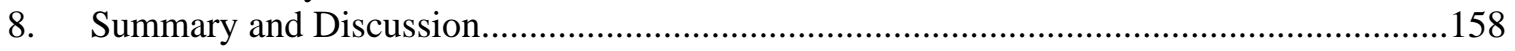

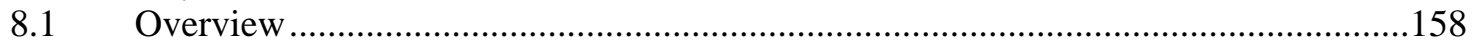

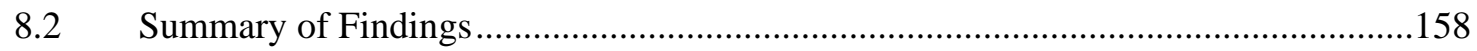

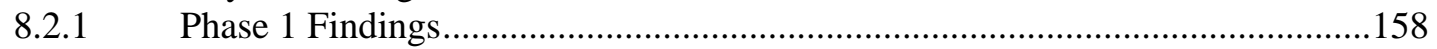

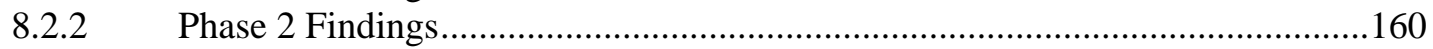

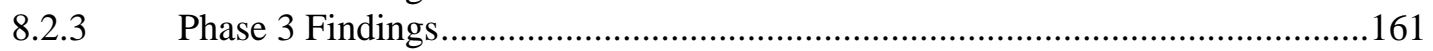




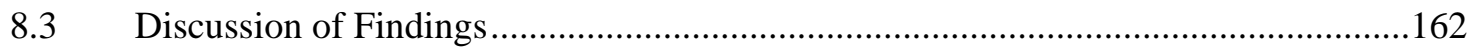

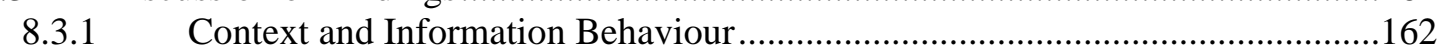

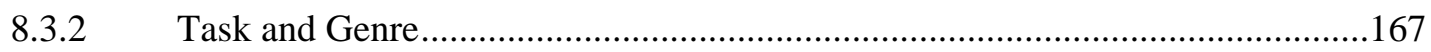

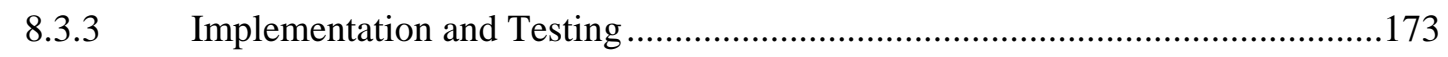

8.3.4 Software Engineers and Workplace Search .....................................................176

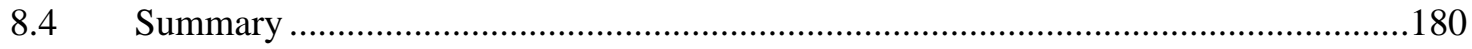

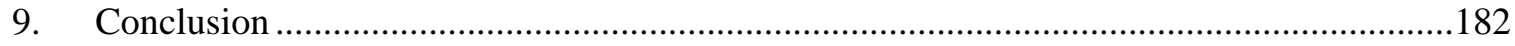

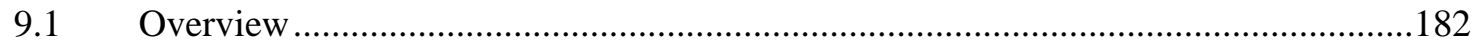

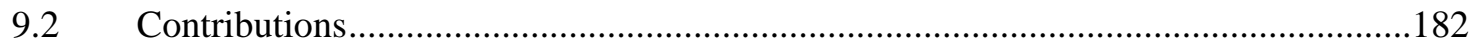

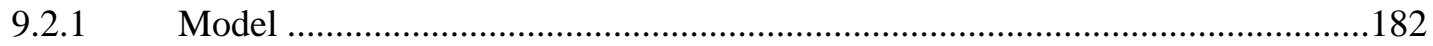

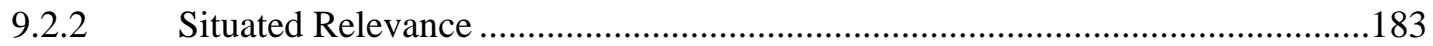

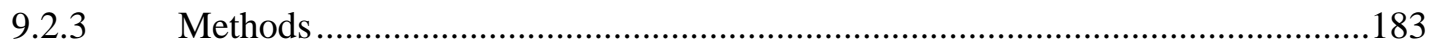

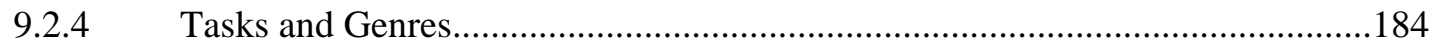

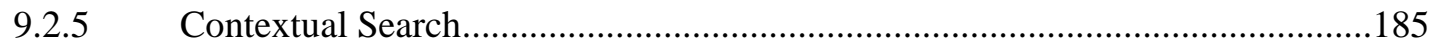

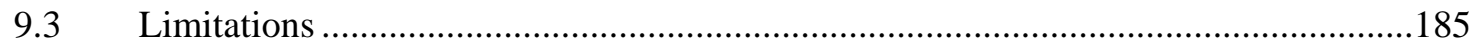

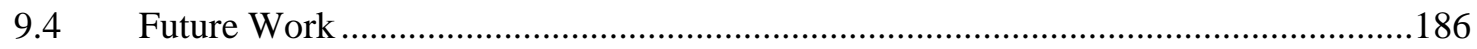

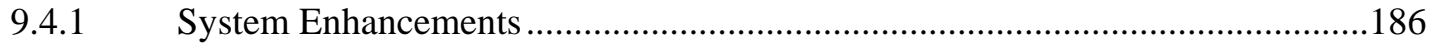

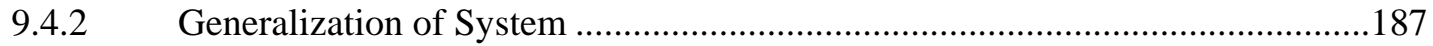

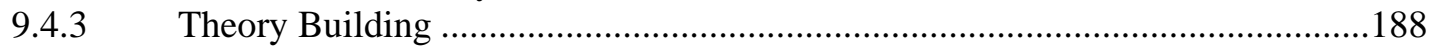

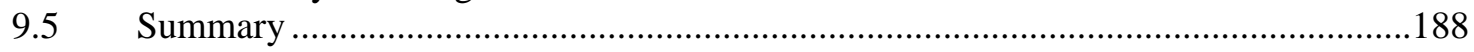

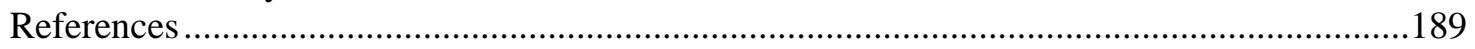




\section{List of Figures}

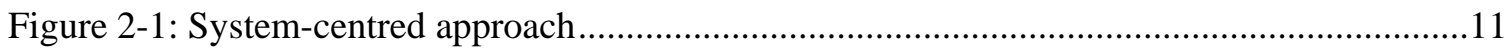

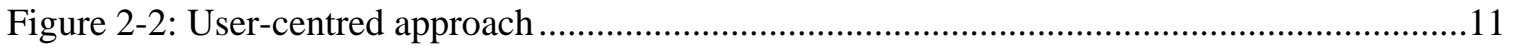

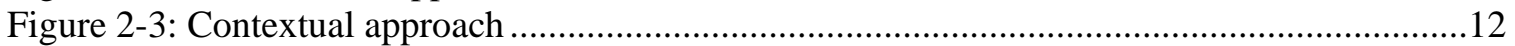

Figure 2-4: General Analytical Model of Information Seeking and Retrieval ..............................18

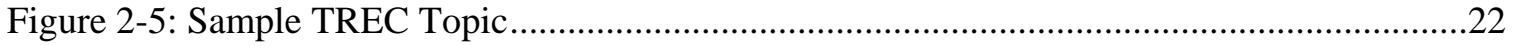

Figure 2-6: Model of the information seeking of professionals .....................................................

Figure 4-1: Model of Contextual Effects on Searching and Selecting Behaviour..........................76

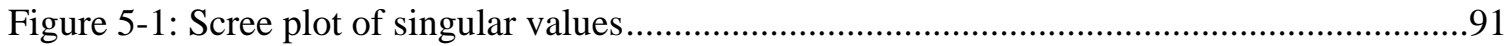

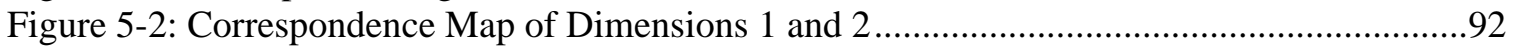

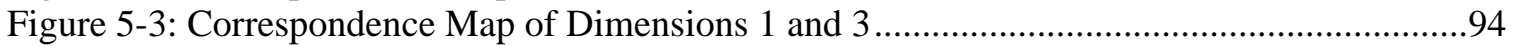

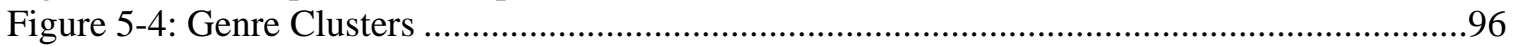

Figure 6-1: X-Site search system components........................................115

Figure 6-2: Distribution of genres for task profile: architecture and fact finding ........................124

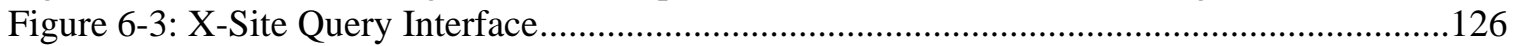

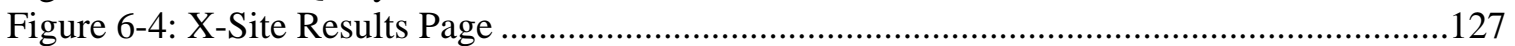

Figure 7-1: Number of relevant documents by search task and system ......................................143

Figure 7-2: Distribution of task completion scores by search task and system ...........................144

Figure 7-3: Distribution of mean rank of relevant documents per search task by system...........145

Figure 7-4: Distribution of usefulness scores of relevant documents by search task ...................145

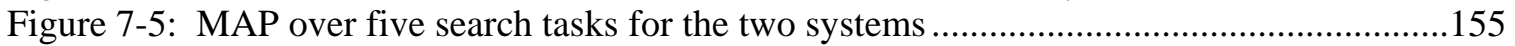

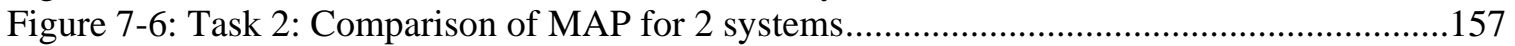

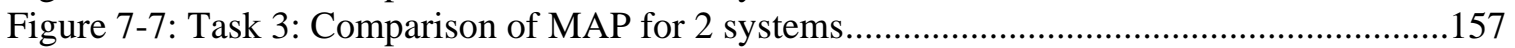

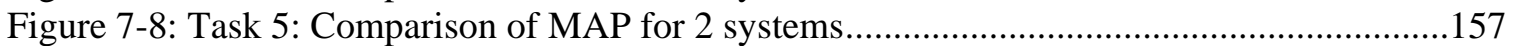

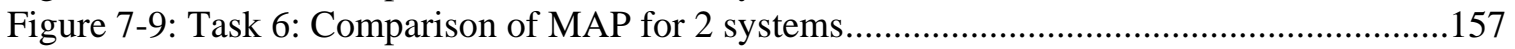

Figure 7-10: Task 7: Comparison of MAP for 2 systems..........................................................157

Figure 8-1: Model of Contextual Effects on Searching and Selecting Behaviour........................165

Figure 8-2: The task-genre relationship mapped onto the General Model of IS\&R ....................171 


\section{List of Tables}

Table 2-1: Summary of selected studies of relevance criteria and cues ....................................26

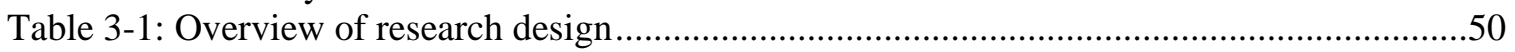

Table 4-1: Common work tasks identified by focus group* ........................................................59

Table 4-2: Key contextual factors influencing searching and selecting behaviour .......................67

Table 5-1: Task and Genre ICR Metadata Categories and Frequencies........................................86

Table 5-2 : Summary of higher and lower than expected co-occurrences of task and genre

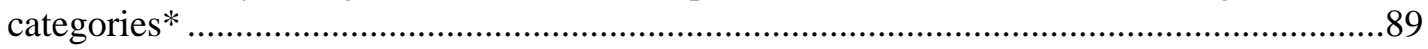

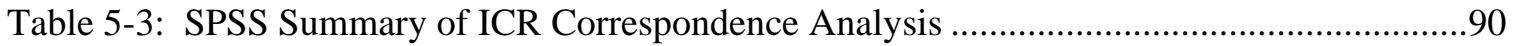

Table 5-4: Work Task and Information Task Taxonomies ....................................................99

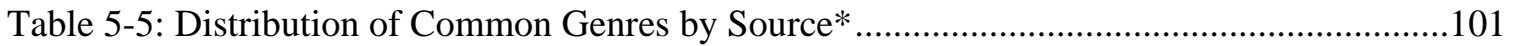

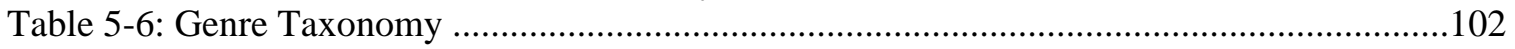

Table 5-7: Queries used to collect Document Sample.................................................................104

Table 5-8: Distribution of Results Classified by Genre Taxonomy ............................................105

Table 5-9: Mean scores and significance tests for characteristics across all tasks .......................109

Table 5-10: Mean scores and significance tests for genres by information tasks.........................111

Table 5-11: Mean Genre Scores by Task …….........................................................................112

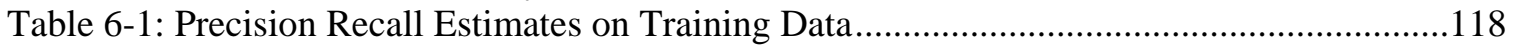

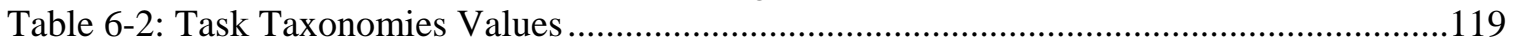

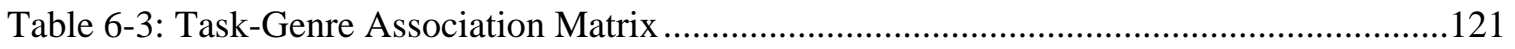

Table 6-4: Summary of Evidence of Associations for Information Tasks and Genres ................122

Table 6-5: Summary of Evidence of Associations for Work Tasks and Genres ..........................123

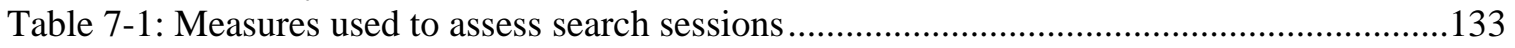

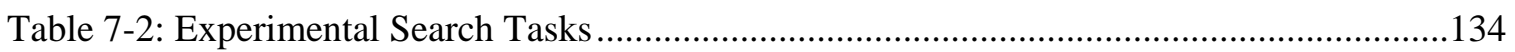

Table 7-3: Experimental Design—System and Search Task Assignments .................................135

Table 7-4: Results of Wilcoxon Signed Rank test for all dependent measures.............................142

Table 8-1: Context-Dependent Variables of Information Behaviour ...........................................159

\section{List of Appendices}

Appendix 1: Workplace Study Consent Form.........................................205

Appendix 2: Workplace Study Interview Script........................................206

Appendix 3: Cross Tabulation of Tasks and Genres......................................209

Appendix 4: Validation Survey E-Mail Advertisement.................................. 214

Appendix 5: Validation Survey Instruments.................................................................215

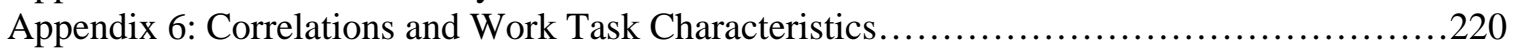

Appendix 7: Evaluation Study E-Mail Advertisement.................................... 221

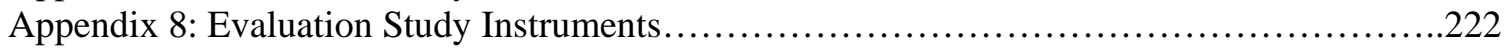

Appendix 9: Evaluation Study Log Sheet for Relevant Documents..........................226

Appendix 10: Evaluation Study Histograms of Dependent Variables..........................227 
We need to free ourselves from the assumption that we can describe information behavior by starting from the system, the service, the knowledge base, or the information carrier. To use these as definers of useful information is misleading: only the recipient, the user, can define information in his or her context. This is not to say that this interface between information system, information artifact and user is not complex and intellectually interesting, but the final determiner of information value is the user who sits in a particular context and develops criteria of information value from that context.

Robert Taylor, 1991

The real impact of information seeking and retrieval is its contribution to the work task processlike effort and time - and the quality of its outcome. Therefore, in the end, information seeking and retrieval should be designed and evaluated for their utility in the work task context.

Järvelin \& Ingwersen, 2004

...biologists know that you only get so far with studying creatures divorced from their ecologies. One might say now that we've learnt quite a lot about the retrieval system creature, and that we need some ecology study in order to design bigger and better bumble bees.

Karen Sparck-Jones, 2006 


\section{Introduction}

\subsection{Overview}

As the amount of digital information on the internet and in other repositories continues to grow exponentially, we find ourselves increasingly dependent upon systems to locate the information we use on an everyday basis. Search technology has become an essential tool of information access, and yet it is still based, at its core, on a sparse mathematical model of text matching rather than on richer models of how people actually interact with and use information in context. As a result, search systems are very effective at retrieving large sets of documents that are "about" a topic, but much human effort is required to sift through these sets to find the smaller number of documents that are useful for the task at hand. This gap between aboutness and usefulness is one of the core problems in information retrieval (IR) (Järvelin, 1986; Järvelin \& Ingwersen, 2004). It is particularly pertinent to workplace environments, where efficient retrieval of useful information is often mission-critical.

In this dissertation, the aboutness-usefulness problem in workplace IR is approached from a contextual perspective, by seeking to identify and exploit variables in the search interaction and environment that influence how people search and what they search for. As elements of the search context, these variables represent the interrelated conditions in which the core IR process the matching of documents to queries - occurs, and they play a role in determining the nature and effectiveness of the search interaction. Of these variables, this research looks specifically at task 
and genre: the work and concomitant information tasks that drive information searching, and the document genres that add shape and meaning to information shared in workplace communities. This introductory chapter frames the problem by describing some of the information access challenges in the workplace, outlines the justification for taking a contextual approach to IR in this domain, and introduces the research questions that guided this research.

\subsection{Workplace Search: more search and less satisfaction}

The information access challenge is particularly acute within organizations. Information is recognized as a valuable capital resource in our information economy, and many organizations have invested heavily in knowledge management practices and technologies that produce, capture and disseminate information. This has resulted in enormous and rapidly growing stores of digital information available for use by organizations. But, as Herbert Simon (1971) suggests, the abundance of information is a double-edged sword: "a wealth of information creates a poverty of attention, and a need to allocate that attention efficiently among the overabundance of information sources that might consume it” (pp. 40-41). As the amount of available information grows, it becomes increasingly difficult for people to find it, make sense of it, organize it, and filter it, which can lead to information overload, a serious issue for organizations worldwide (D. Allen \& Wilson, 2003; Waddington, 1996). The resulting paradox, is that the surfeit of information results in a paucity of useful information, because the latter becomes much more difficult to find (Edmunds \& Morris, 2000). Far from decreasing in recent years, information overload has been named "problem of the year” for 2008 by the business research firm Basex (Lohr, 2007).

Current workplace search systems are not effective tools in the face of these information access challenges. Knowledge workers, professionals whose work deals with the production, manipulation, use and sale of information (Drucker, 1959), spend a significant amount of their 
time (15-35\%) searching for information, but are successful only about 50\% of the time (Feldman, 2004). These are not typical Internet search engine users: knowledge workers tend to be highly motivated professionals with domain experience, and they are typically willing to invest significant amounts of time formulating search queries, using advanced system features, and evaluating sources (Choo, Detlor, \& Turnbull, 2000c). However, tools to support this type of focused, domain-specific and strategic searching are not available in most workplaces. According to Kirsh (2000), “more search and less satisfaction,” is the result of reliance upon search tools that do a poor job of gauging information quality and relevance: "no search engine seems to return hits with sufficient precision to save us from having to browse dozens of useless pages in our effort to berry pick the best items” (pp. 24-25). This situation results in a huge drain on resources within organizations, both in terms of time spent searching and the costs associated with duplicated work and errors when information is not found (Feldman, 2004).

\subsection{Why Contextual Search?}

Traditional IR systems make use of generic, statistical and text-based techniques to match documents with queries. This approach is inherently limited because it is not based on the way that people actually look for and use information in context, in the framework of real activities and settings. Keyword queries are taken to represent human information needs, despite the fact that we know that queries tend to be very brief (Spink, Jansen, Wolfram, \& Saracevic, 2002), inaccurate and/or incomplete representations of such needs (Ingwersen, 1992; R. S. Taylor, 1968). We also know that when people evaluate and select information resources, they judge relevance not only based on topical content, but also based on a range of other dimensions: social, situational, cognitive and affective (Borlund, 2003a; Saracevic, 1996; Schamber, Eisenberg, \& Nilan, 1990). For example, information about global warming may be judged "irrelevant” to a search on global warming if the information content is already known or 
incomprehensible to the searcher, if it is poorly formatted and difficult to assess, or if the style and underlying purpose of the text is inappropriate for the searcher's intended use of it.

The role that context plays in shaping how people search for, select and use information has been recognized and described at a high level in key models of information behaviour (Dervin, 1999; T.D. Wilson, 2000) and interactive IR (Järvelin \& Ingwersen, 2004; Saracevic, 1996). Taylor (1986) noted two decades ago that information needs consist of two distinct but inter-linked parts: the topic or subject matter of the need, and the situational factors that motivate and influence how information will be used. Furthermore, context seems to have an important influence over patterns of information behavior in the workplace. Different patterns of information behaviour have been identified for different types of work domains (Järvelin, 1986; R. S. Taylor, 1991), different types of tasks (Byström, 2002) and at different stages of task completion (Vakkari \& Hakala, 2001). What this suggests is the potential for IR systems to better accommodate the particular, situated information needs of searchers, by augmenting the matching algorithms of retrieval systems with relationships between contextual variables. However, despite these strong indications that information behaviour is context dependent little work has been done to isolate and validate relationships between specific contextual variables, and as a result this work has had surprisingly little impact on search system design (Järvelin \& Ingwersen, 2004).

Accounting for the role of context has been identified as one of the key challenges in IR research (Allan et al., 2003). A contextual approach to IR looks beyond the subject matter and textual content of queries and documents, to examine variables in the search environment that influence how people search as well as the nature of the information they seek, with the aim of improving the quality and precision of search results. More specifically, this means attaining "higher order relevance" by retrieving information that meets user needs more fully, by supporting problem solving and task completion (Järvelin \& Ingwersen, 2004). 


\subsection{Research Objectives}

On a practical level, this work was motivated by the need for more discriminating and effective workplace search tools. On a theoretical level, it was motivated by the incongruous gap between models of human information behaviour in which context plays a major role, and models of IR in which context is virtually ignored. This research set out to bridge that gap by identifying the contextual factors that influence search and making use of them to increase the realism of the IR model and to develop more effective search tools. Beginning with a detailed study of contextual factors in a workplace setting this research then focused on the relationship between a subset of the identified factors: the tasks undertaken by searchers and the genres of documents in the information environment. The task-genre relationship was explored, characterized and tested as a means of identifying pragmatic, use-centred associations between information objects and searchers. One method of implementing task-genre relationships was tested in a live workplace search system.

The study progressed from an exploratory initial phase through a series of separate studies to validate and test the relationship between task and genre. To follow are the broad research objectives that guided the project from the outset. More specific research questions were formulated for the various phases of the research and are described in the relevant chapters to follow.

1. To identify and model the role of context in shaping the searching and selecting behaviour of software engineers in a workplace environment.

Patterns of information behaviour exist within professional domains and work settings, as evidenced by the large number of studies that describe the information seeking behaviour of various groups as reviewed by Donald Case (Case, 2002, 2006). While studies of engineers as a professional community serve as a starting point for this research (Tenopir \& King, 2004), little 
has been written specifically about software engineers working within a completely digital information environment. Therefore, it was necessary to approach this objective from the bottomup to determine the contextual factors that are most influential. The outcome of this objective was a model of selected contextual factors and their effects upon searching and selecting behaviour.

\section{To identify, characterize and operationalize a subset of inter-related contextual factors that have a significant influence on searching and selecting behaviour.}

The general model of contextual factors and effects developed in the first phase of research identified many factors and types of effects, which could not be tested all together without confounding the results. Therefore, it was necessary to extract from those results a subset of factors with a strong effect on searching. The strongest candidate factors identified in the first phase of the research were work tasks, information tasks and genres. The second phase of research focused on testing and more-fully characterizing the nature of the relationships between tasks and genres, and operationalizing these variables as domain-specific taxonomies. This phase of the research produced evidence of a significant association between these factors, taxonomies of values for each, and evidence to suggest associations between specific task and genre values.

\section{To evaluate the use of task-genre relationships in a search system.}

The third phase of this research was aimed at determining a means of extending the traditional IR model to incorporate task-genre relationships, and testing an implementation of these relationships in a live search system. During this phase of the research, the relationships between tasks and genres were formalized into a set of contextual components that were incorporated into a retrieval system. Together the components functioned as a task-based genre filter on search results. The contribution to search effectiveness of this implementation of task-genre relationships was evaluated in a controlled user study conducted in the field. 


\subsection{Definitions}

For clarity, this section defines some of the terms frequently used in this dissertation.

Information Retrieval (IR) can be defined from a systems perspective as: "the techniques of storing and recovering and often disseminating recorded data especially through the use of a computerized system ${ }^{1 »}$. However, this research takes a combined human and systems approach, in which the terms $I R$ and search are used interchangeably to refer to the broader interaction process that takes place when a person makes use of an IR system to search for and retrieve information objects. When the focus is extended to include non-system based information seeking, the term Information Seeking and Retrieval (IS\&R) will be used. Workplace IR refers to the practices and technologies associated with on-the-job information seeking using computerized systems. The focus of workplace search is on organizational information spaces and the information needs and practices of employees in the course of their work.

Information Objects are physical or digital entities, which use textual, audio, pictorial or other forms of content to provide information to users. Throughout this dissertation, the term document is meant in the broader sense of information object. A related term, information source, refers to a container (repository, website, database) in which multiple information objects are stored and through with they are made accessible.

Context, defined broadly, refers to the "interrelated conditions in which something exists or occurs ${ }^{11,}$. In the case of search context, it can be defined as the interrelated conditions in which the core IR process, the matching of documents to queries, exists, and which play a role in determining the nature and effectiveness of the search interaction. Contextual factors are the isolated elements of context that function as independent variables, playing a role in shaping the search interaction. This research focuses primarily on three contextual factors: work tasks,

\footnotetext{
${ }^{1}$ Merriam-Webster Online, http://www.merriam-webster.com/dictionary. Accessed February 18, 2008.
} 
information tasks and genre. In general, tasks are activities undertaken to achieve goals. In the software engineering domain, work tasks are assigned professional activities, such as installation and migration, which fall into well-known categories based on common goals and methods. Information tasks, which are prompted by work tasks, are activities related to the acquisition and use of information that can be grouped according to associated cognitive goals, such as learning, deciding, problem solving, etc. Genre is a composite feature of information objects based on aspects of form, purpose and content. Following functional genre theory, genre types such as technical articles or whitepapers are "socially recognized types of communicative actions [...] that are habitually enacted by members of a community to realize particular purposes” (Yates, Orlikowski, \& Okamura, 1999).

\subsection{Summary and Structure of Dissertation}

This research was conducted in multiple phases, each one building on the findings of the previous phase. This research design is reflected in the dissertation structure.

Chapter Two situates the contextual approach with respect to more traditional approaches to IR and provides a review of related theoretical and empirical work.

Chapter Three provides a brief overview of the research design, study setting and population.

Chapter Four reports on a study of a software engineering workplace domain which identifies the contextual factors and their role in shaping information behaviour.

Chapter Five reports on a series of studies conducted to validate and explore the relationship between task and genre within the study setting.

Chapter Six provides an overview of the implementation of task-genre relationships in the X-Site search system. 
Chapter Seven reports on studies conducted to evaluate the contextual components implemented in X-Site.

Chapter Eight provides a summary and discussion of the main findings.

Chapter Nine identifies the contributions, discusses limitations and outlines future work. 


\section{Previous Research}

\subsection{Overview}

This chapter provides the background for this research by characterizing the contextual approach to IR, and situating it as an extension of the user-centred cognitive perspective, which stands in contrast to the system-centred research tradition in IR. Previous work in the areas of human information needs, relevance and information behaviour is presented as motivation for adopting a contextual approach to IR, and literature on topics relevant to this research is explored: workplace search, information behaviour of engineers, and task and genre as information seeking and retrieval (IS\&R) variables. The chapter begins with a discussion of different theoretical approaches to IR.

\subsection{Approaches to IR}

IR occurs when a person uses a system to find information. This human-system-information interaction can be modeled very simply as an input-output transaction. However, an almost infinite range of variables associated with the person, the system, the information and the interaction may influence IR activity over time, and IR research has been moving in the direction of increasingly contextualized models. Examples of this system-centered, user-centered and contextual approach to IR are illustrated by Figures 2-1 to 2-3 below. 
The system-centred approach (Figure 2-1) focuses on the system and the information, independent of the contexts in which they are created and used. In this model, the user is represented by a generic user surrogate who is positioned outside of the loop.

Figure 2-1: System-centred approach

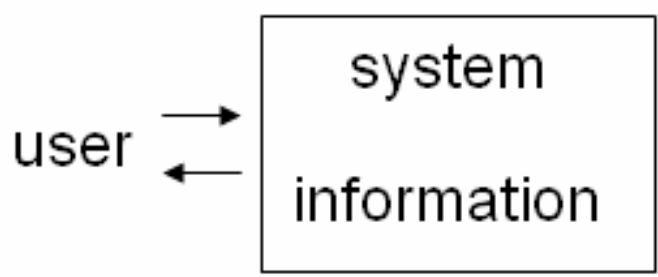

The user-centred approach (Figure 2-2) focuses on the user's interaction with a system, from within the context of the user's personal characteristics, his or her emotional state and and the socio-cultural context.

Figure 2-2: User-centred approach

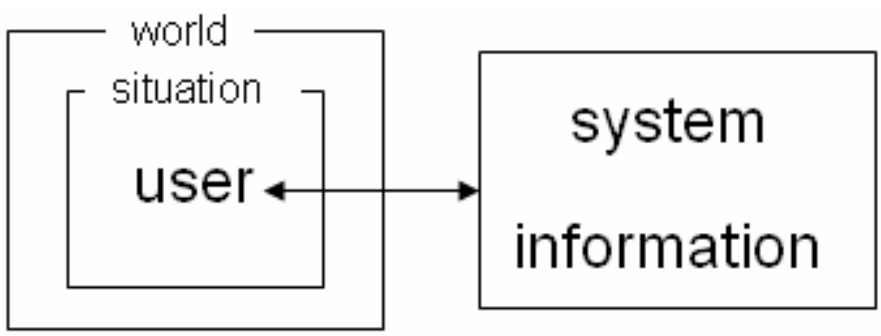

Finally, the contextual approach (figure 2-3) considers the interaction of all three components within an environment made up of static and dynamic contexts (the world and the situation) that influence the nature of the components and the nature and value of the interaction. 
Figure 2-3: Contextual approach

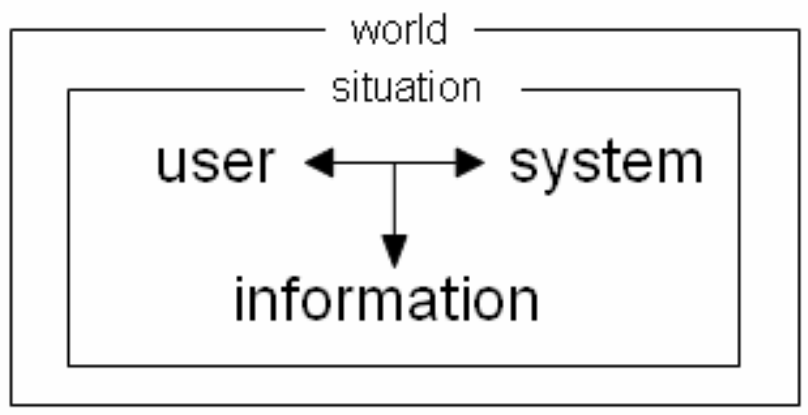

The following three sections will describe each of these approaches in more detail, in order to clarify what is meant by the contextual approach, justify its adoption, and situate it within the IR research tradition.

\subsubsection{System-Centred Approach}

Within IR research, the system-centered approach has a long tradition with a cohesive body of empirical research. This approach focuses on the development of theories, algorithms and techniques to improve retrieval performance, i.e., the capability of a system to retrieve information that is relevant to queries. The theoretical and empirical underpinnings of systemcentred IR have been described in numerous monographs and texts (See for example Baeza-Yates \& Ribeiro-Neto, 1999; Salton, 1989; van Rijsbergen, 1979). In this research paradigm, relevance is interpreted as topical congruence, and is operationalized in various retrieval models as some sort of relationship (similarity, proximity, connectivity, etc.) between queries and information objects (Sparck-Jones, 2003). Thus, a key focal point of this research has been to develop effective matching algorithms. Retrieval performance is typically measured using two metrics: precision (ratio of relevant documents retrieved to total retrieved) and recall (ratio of relevant documents retrieved to total relevant documents in database), with the goal being a system that retrieves: "all the relevant documents [and] at the same time retrieving as few of the non-relevant as possible” (van Rijsbergen, 1979, p.6). 
This approach to IR was developed in the early 1960s as a result of a series of experiments designed to develop and evaluate automatic indexing methods, lead by Cyril Cleverdon (1960; 1966). The Cranfield experiments established the tradition of evaluating retrieval systems and techniques using test collections consisting of sets of documents, sets of topics (queries) and sets of static human judgments on the relevance of documents for each topic. Cleverdon made a conscious choice to exclude contextual variables from these evaluations, which did not include real-life searchers, tasks or situations. He did this to increase his control over the environment in which the experiments were conducted and thus to render his testing methods more "scientific" (Ellis, 1992). The value of this "scientific" approach to IR is that it has succeeded in supporting systematic, incremental improvements to the design of retrieval technologies (Salton, 1996; Sparck-Jones, 2003). This evaluation framework continues to be used in the Text Retrieval Conferences (TREC), one of the main venues for system-centred IR research (Voorhees \& Harman, 2005) and in the Initiative for the Evaluation of XML Retrieval (INEX).

However, the weakness of this abstract and algorithmic approach to IR is that although findings can be considered valid within the framework of the model that drives the research (Kekäläinen \& Järvelin, 2002; Salton, 1992) they are limited with respect to meeting users’ needs in realworld applications. This is due to the fact that the system-centred approach rests on a number of highly questionable assumptions:

- A (single) query is an accurate representation of an individual's information need;

- A (single) query is an accurate representation of the same information need held by a number of people;

- $\quad$ Relevance is based only on the subject matter or topic, and is a static, binary concept that can be assessed objectively;

- An ideal IR system should retrieve everything that is relevant, and only that which is relevant;

- $\quad$ IR is essentially an input-output (transactional) problem. 
This approach has been challenged by a strong counter-swell of user-centred research critiquing the traditional IR model (See for example Belkin, Oddy, \& Brooks, 1982a; Ingwersen, 1992), suggesting that the model and evaluation framework needs to be extended to incorporate a more complex understanding of the IR process and the human needs that drive it (i.a. Borlund, 2003b; Järvelin \& Ingwersen, 2004; Kekäläinen \& Järvelin, 2002). More recently, researchers who have worked from within the system-centred paradigm have also begun to agree that moving IR research forward requires "facing up to context” (Allan et al., 2003; Jose \& van Rijsbergen, 2005; Sparck-Jones, 2006)

\subsubsection{User-Centred Approach}

During the 1980s, alternate "user-centred” perspectives began to emerge in response to the lack of realism in the core assumptions of the system-centered approach to IR and other information science disciplines. Although user-centred IR studies do not represent a cohesive body of research, they share a common focus on the human information seeker, his or her perception and understanding of the world, and the nature of his or her interaction with the system. A much cited review (Dervin \& Nilan, 1986) characterized this approach as a fundamental paradigm shift along multiple dimensions: from objective to subjective approaches; from passive to active users; from trans-situational to situational approaches, and from an emphasis on external behaviour to internal cognition. Robertson and Hancock-Beaulieu (1992) identified three user-centred IR “revolutions" taking place in the research arena, related to relevance, cognition and interactivity, which brought into question the validity of assuming that relevance could be judged based on a document-query match, and that IR systems could be evaluated without taking into account the increasingly interactive nature of searching.

The theoretical perspective most strongly associated with the user-centred approach to IR is the cognitive viewpoint. The cognitive perspective views human beings as agents in a dynamic process of interpreting the world through personal mental constructs, or cognitive frames, that 
reflect the worldview of each agent. It was first applied to information science in the early 1980s (Belkin, 1980; de Mey, 1980; Ingwersen, 1984; T. D. Wilson, 1984) and gained influence through later work in the field of interactive IR (Belkin, 1990; Ingwersen, 1992). From the cognitive perspective, an IR system can be seen as a locus of interaction for the cognitive structures of the agents involved; a type of communication system in which meaning is not inherent in the message, but is "synthesized by the receiver out of his own knowledge” (de Mey, 1980, p. 54). The cognitive approach forms the basis for Ingwersen's Model of Interactive IR (Ingwersen, 1992, 1996), the Anomalous State of Knowledge (ASK) model (Belkin et al., 1982a; Belkin, Oddy, \& Brooks, 1982b) , and Saracevic’s Stratified Model (Saracevic, 1996), all of which represent IR as a dialog, in which relevance, meaning and value are context-dependent, subjective and dynamic constructs.

The user-centred approach to IR also has been influenced by models of human information behaviour, which emphasize the broader context of the seeker. Allen’s (1997) “person in situation” model emphasises the importance of situations, social settings and groups on individual and group information needs. Wilson's $(1997,1999)$ model of information behaviour describes a wide range of physiological, affective and cognitive factors, as well as the roles people play in the world which affect information seeking behaviour as it unfolds. Dervin’s $(1997,1999)$ sensemaking framework similarly emphasizes the role of the seeker in the process of making sense of their world. Dervin's famous “gap” scenario, of a person standing at the edge of a gully trying to find a means of reaching the other side, serves as a powerful metaphor for user-centred information seeking. However, equally important to Dervin's framework is its emphasis on the situatedness of searchers: their positions in the flux of time and space, personal and emotive states and motivations for seeking information. These models suggest that a person's information behaviour is influenced by relatively static contextual factors such as their cognitive abilities and cultural environment, as well as by changing, situational factors which may differ from one 
information seeking incident to the next, such as the searcher's emotional state, the questions they need answered, or the activities in which they are engaged.

These "user in context" models of information behaviour inspired a large body of research focused on studying information behaviour among diverse populations (Case, 2002, 2006; Vakkari, Savolainen, \& Dervin, 1997) and the impact of individual differences on searching behaviour (B. Allen \& Kim, 2000; Kuhlthau, 1993; Savage-Knepshield \& Belkin, 1999; Wang, Hawk, \& Tenopir, 2000). However, the user-centred approach is not easily applied to the central problem of IR systems, i.e. the matching of documents to requests. Users, like systems, are only one component of the IR interaction, and the strong emphasis on individual cognitive and emotional differences between system users is difficult to accommodate in designing for large user communities.

Furthermore, the models produced by much of this research are primarily descriptive rather than analytical models; they describe the seekers, the information needs and some of the attributes of each in various contexts, but do not identify relationships among these components (Järvelin \& Wilson, 2003; J. D. Johnson, 2003; Talja, Keso, \& Pietilainen, 1999). The difficulty of deriving testable hypotheses from these models means that they have had little direct impact on the design of retrieval systems, despite the fact that they have had a major impact on our understanding of how people interact with IR systems.

\subsubsection{Contextual Approach}

In recent years, a contextual approach to IR has begun to emerge due to the recognition that although modeling the broader ecology of IR system use poses certain challenges, it also offers the potential of further improvements in retrieval accuracy (Allan et al., 2003; Sparck-Jones, 2006). The motivation for taking a contextual approach is pragmatic and system-oriented: "The underlying hypothesis (belief) is that by taking into account of context the next generation of 
retrieval engines dependent on models of context can be created, designed and developed delivering performance exceeding that of out-of-context engines” (Ingwersen, Järvelin, \& Belkin, 2005).

The contextual approach is an extension of the cognitive user-centred approach and it draws heavily upon user-centred models of information behaviour and information interaction developed in the mid 1990s. However, it is also influenced by more situated and pragmatic approaches, such as domain analysis (Hjorland \& Albrechtsen, 1995), cognitive work analysis (Fidel \& Pejtersen, 2004) and activity theory (Bodker, 1991), which focus on human tasks and activities as they occur within social and physical environments. Thus, while the contextual approach is not yet well-defined, a common thread of goals and issues can be identified:

- It aims to bridge the conceptual and methodological gaps between human information behaviour research and system-centred IR in order to apply more complex and realistic models to system design.

- It aims to extend existing IR models and methods of evaluation beyond the point of document retrieval, to include the use of information in real world settings for problem solving and task completion.

- It takes an analytic and pragmatic rather than holistic and descriptive approach to context.

- It emphasizes the temporal, process-oriented and interactive aspects of IR.

- It incorporates both cognitive and socio-cognitive perspectives on information behaviour.

- It recognizes that generic models of IR are inherently limited and that there is value in developing specialized IR tools or techniques for particular settings, populations or situations. 
The first challenge in delineating a contextual approach is to define the related concepts of context and situation. In the introduction, search context was defined as the interrelated conditions in which the core IR process exists, and which play a role in determining the nature and effectiveness of the search interaction. The central pre-occupation of the contextual approach to date has been to disentangle these "interrelated conditions" into a set of named elements or dimensions and to identify the variables that make up each element. In the most thorough discussion of a contextual approach to IR to date, Ingwersen and Järvelin (2005) propose a model (Figure 2-5) that extends Ingwersen's earlier work (1996). It defines five main elements that make up the IR interaction: cognitive actors; organizational, cultural and social context; the interface, information objects, information technology, and possible interactions between elements.

Figure 2-4: General Analytical Model of Information Seeking and Retrieval (Ingwersen \& Järvelin, 2005)

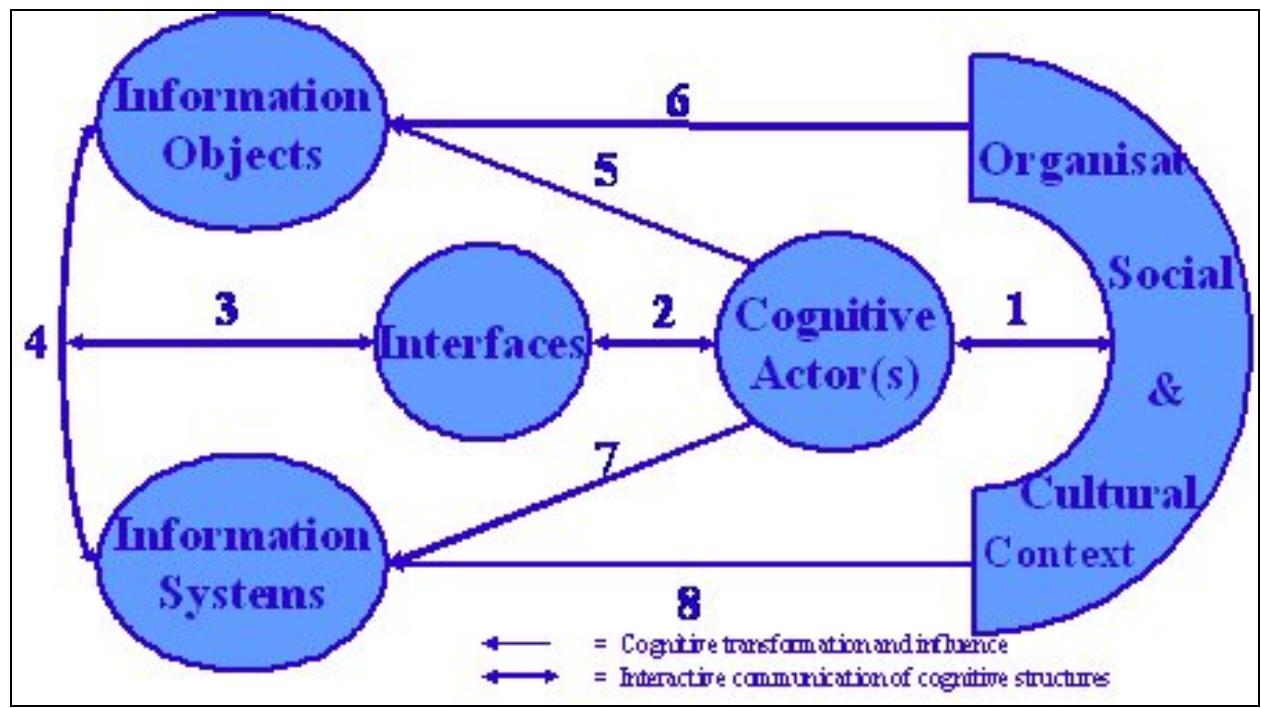

The authors suggest that each and all these elements contextualize each other (inter-object context). For example, the cognitive actors involved in a search session, searchers, authors, indexers, system designers, etc., provide context which influences the information objects, the technology, the social context, etc. Intra-object structures also influence the interaction from 
within each of the five elements, such as the level of domain knowledge of the searcher, and the usability of the interface. Three addition contextual elements are identified in the model, which are not shown in the diagram. These are: the features of the interaction between the elements; temporal features, and work task or interest features (Ingwersen, 2005). Alternate schemas to map contextual variables have been proposed (Toms, Freund, Bartlett, \& Szigeti, 2004), which differ to some extent about the boundaries of elements and their relative importance (Cool \& Spink, 2002). However, this model includes generally agreed upon core elements: users; systems; information objects; social/cultural/physical environments; tasks and goals; interactions; and time.

A related concern has been to refine this general model of IS\&R context to represent particular domains or user groups. For example, a proposed contextual model for the field of evidencebased medicine consists of the elements: work tasks, search tasks, problem structures, processes and the medical domain (Lin \& Demner-Fushman, 2006) . A mixed-method study of information practices in a large aerospace company identified: information flow, information types, users and tasks, tools and logistics, perceptions and actions as the key elements (Petrelli, Lanfranchi, Moore, Ciravegna, \& Cadnas, 2006). A study of doctoral student researchers found four significant contextual factors: task, type of problem, accessibility and research community (Chang \& Yee, 2001). While some of the elements overlap, notably tasks, the importance of different elements of context seems to vary across domains.

A concept closely related to context is situation. In a search situation, a particular person interacts with a particular information system for a particular purpose, under particular conditions at a given time and place (Cool, 1997, 2001). The concept of situation has been used in information science in various ways (Cool, 2001), including Allen’s “person in situation” model . A common approach is to consider the more dynamic elements of a search, the problem, the information need, the system, etc. as aspects of the "search situation"; whereas other more static elements 
such as the setting and the searcher's characteristics are treated as “context”. However, proponents of the contextual approach tend to view a situation as the set of values of all the contextual variables at play at a given point-in-time (Ingwersen \& Järvelin, 2005; O'Brien \& Toms, 2007) . Kelly (2006) proposes that the complexity of this type of situation can best be represented by a matrix structure, composed of all the variables affecting the search and their values. This view of situation is useful, in that it allows for the search interaction to be represented as a time-series of situations, throughout which some contextual elements change, while others do not. By studying this situational variation, contextual models can be developed both at the relatively static level of domains and user groups and at the more dynamic level of discrete tasks and interactions.

Alongside the ontological challenges of defining context, a more pragmatic concern has been to identify which variables have the strongest effect and can be captured and exploited in a system, i.e. those which give the most "bang for buck"(Hawking, 2005). Research findings on this question are growing, but results still tend to be scattered and difficult to synthesize due to differences between settings, definitions, and research goals. For example a range of studies on task context have shown that task variables (task types, time constraints, importance, stage of completion, complexity, etc.) influence various aspects of information behaviour (relevance criteria, usefulness judgements, selection of channels, number and type of sources consulted, cognitive activities, etc). (Algon, 1999; Byström, 2002; Byström \& Hansen, 2005; Cosijn, 2006; Kelly, Murdock, Yuan, Croft, \& Belkin, 2002; Vakkari, 2003). Some of these findings will be discussed in more detail in the section on tasks (2.6) below. While these results contribute to our understanding of contextual effects, there are still very few examples of these types of findings being incorporated into a retrieval model and tested in a system framework. Thus, the contextual approach, while promising, has not yet made a significant contribution to the improvement of search systems. 


\subsubsection{Summary}

The three approaches to IR described here represent a continuum from a highly-focused and decontextualized model of IR to a model which is broader along a number of dimensions. The contextual approach considers a larger number of variables, focuses on a broader spectrum of activities and examines them at a finer level of granularity. However, these three approaches to IR are not discrete, as the contextual approach represents an attempt to both integrate and extend the user-centred and system-centred approaches. Whether the exploitation of context will make a real contribution to IR systems remains to be seen. Most of the work to date has focused on understanding the nature and role of context and little has been done to exploit and evaluate its effect in a system setting.

\subsection{Context and Human Information Behaviour}

One of the key assumptions behind the contextual approach in general and this research in particular, is that context motivates and shapes human information behaviour and can therefore be used to interpret and/or predict behaviour. Research and practice in the field of information science supports this assumption with respect to two aspects of information behaviour: information needs and relevance assessments.

\subsubsection{Information Needs}

Information needs are often seen as the force behind information seeking (Case, 2002). In the system-centred paradigm, exemplified by the TREC evaluations, information needs are represented by detailed topic statements (Figure 2-5). However, user-centred studies of IS\&R indicate that using these detailed de-contextualized topic descriptions as surrogates for actual user needs is untenable. Studies of library users during reference transactions found that actual information needs are often the product of muddled and incomplete understandings of topics and tend to be poorly expressed by searchers (Ingwersen, 1992; R. S. Taylor, 1968). Belkin (1980) 
coined the expression Anomalous State of Knowledge (ASK) to describe the problem faced by most searchers who do not know what they are looking for and therefore are ill-equipped to describe it. Even when searchers are capable of describing their need in detail, Ingwersen noted the tendency to under-specify, which he called the "label effect" (Ingwersen, 1982). Furthermore, human information needs are experienced along a number of dimensions: cognitive, affective and situational (Choo, Detlor, \& Turnbull, 2000a) and thus cannot be reduced to a topic statement. As Allen (1996) noted, “information needs happen to individuals who are embedded in a range of social situations” (p. 88).

Figure 2-5: Sample TREC Topic

Title: Behavioural Genetics

Description: What is happening in the field of behavioural genetics, the study of the relative influence of genetic and environmental factors on an individual's behaviour or personality?

Narrative: Documents describing genetic or environmental factors relating to understanding and preventing substance abuse and addictions are relevant. Documents pertaining to attention deficit disorders tied in with genetics are also relevant, as are genetic disorders affecting hearing or muscles. The genome project is relevant when tied in with behaviour disorders (i.e., mood disorders, Alzheimer's disease). (topic 402, LA Times Collection)

An alternate approach to information need elicitation was proposed by Belkin, Oddy and Brooks (1982b). The approach was based on the idea that information needs arise in the framework of problematic situations, and that asking searchers to describe their situation, current state of knowledge, motivation and intended use of the information would elicit a different type of need representation. They designed an experimental system in which lengthy user statements describing information problems were used to construct word association networks to serve as structured search requests.

While rare in operational search systems, this approach to describing information needs in context is commonplace in the library world. In the reference interaction, for example, an information professional may attempt to clarify a request for information by prompting an information seeker for details about the context of his or her information need. Taylor (1968) suggested that five 
clarification "filters" be used to negotiate reference questions with the searcher: the topic, the searcher's objectives and motivation, personal characteristics of the enquirer, relationship of the inquiry to the file structure, and acceptable answers. Similarly, the goal of the neutral questioning approach to the reference interview is to elicit the searcher's motivation and intended use of the information (Dervin \& Dewdney, 1986). In a novel comparative study of intermediated and nonintermediated searching, Nordlie (1999) showed that knowing why information is needed gives human intermediated searching an advantage over end-user searching on Online Public Access catalogues.

Although attempts have been made to model expert retrieval systems on the intermediation process used by information professionals to clarify information needs, these have not met with great success (Belkin, Seeger, \& Wersig, 1983; Ingwersen, 1992, chapter 8) . Nevertheless, a strong body of evidence points to the importance of contextual factors in motivating and clarifying human information needs and in identifying sources capable of satisfying those needs.

\subsubsection{Relevance}

Relevance, the quality of one thing having a direct bearing on another, is a fundamental concept in IS\&R. As the flip side of information needs, relevance provides the basis for determining what information is likely to satisfy those needs, and thus what information is worthy of retrieval. Whereas system-centred IR predicts relevance based on objective measures of topical similarity, the user-centred approach considers relevance to be a function of the applicability of information to a user's need, problem, situation and context, based on the user's subjective judgment. This idea of two relevances: topical relevance, or aboutness, versus situational relevance, is the subject of a long-standing debate (Cooper, 1971; P. Wilson, 1973) and is an important motivating factor for this research. 
From a user-centred perspective, relevance is a dynamic, situated and multi-dimensional concept (Borlund, 2003a; Cosijn \& Ingwersen, 2000; Mizzaro, 1998; T. K. Park, 1994; Schamber, Eisenberg, \& Nilan, 1990). Saracevic $(1975,1996)$ defined five types of relevance that underscore the IR interaction: system (algorithmic) relevance based on the similarity of a query to a document; topical relevance (subject similarity); cognitive relevance (pertinence) based on the fit between a document and a searcher's state of knowledge at a given point in time; motivational (affective) relevance based on the fit between information and a user's intentions, goals and motives, and situational relevance (utility): the fit between the situation, problem space or task of the user and the information.

Subsequent studies (Borlund, 2003a; Cosijn \& Ingwersen, 2000; Mizzaro, 1998; Toms, O'Brien, Kopak, \& Freund, 2005) have proposed variations on this typology of relevance; however, they all include some form of situational or task-based relevance. Borlund (2003a) defined situational relevance as a "user-centred, empirically based and realistic as well as potentially dynamic type of relevance. Situational relevance expresses the relationship between the user's perception of usefulness of a retrieved information object, and a specific work task situation”. Mizzaro’s (1998) multi-dimensional model of relevance also emphasizes the role of tasks and context in IR, which the author notes have been understudied to date. Mizzaro goes so far as to suggest that, “one could build a system, that starting from some characteristics of the task, infers the desired characteristics of the information” (p. 13). This approach is followed in this research to test the relationship between tasks and document genres.

In addition to situational relevance, Cosijn and Ingwersen (2000) propose that relevance has a socio-cognitive dimension: "the relation between the situation, work task or problem at hand in a given socio-cultural context and the information objects, as perceived by one or several cognitive agents”. Socio-cognitive relevance expresses the influence that communities, cultures, and organizational frameworks play in determining information value. It is strongly associated with 
the idea pursued in this research, that IS\&R behaviours follow context-dependent patterns within defined professional domains and communities.

\section{i. Relevance Criteria}

Just as context can be represented as a set of interrelated factors, relevance can be represented as a set of criteria used by searchers to evaluate information objects. Numerous empirical studies of how people assess relevance have identified a large pool of criteria, many of which relate to nontopical characteristics and features of information objects, as well as to aspects of the searcher and the situation (Barry, 1994; S. Y. Park, 1993; Schamber, 1991; Tombros, Ruthven, \& Jose, 2005; Wang, 1994).

Table 2-1 shows a compilation of categories and criteria from selected studies, together with a brief summary of the research method and the study domain for each. Considerable overlaps in the criteria across studies suggest that strong general patterns exist across domains. A recent meta-ethnography of sixteen relevance studies was able to reduce 133 user criteria to 14 consolidated labels, including: recentness, accessibility, quality, novelty, affordability, intelligibility, and saturation (Bales \& Wang, 2005). However, there is considerable variation in the way these criteria are grouped and presented in the different studies. Park (1993) and Barry (1994) mix together document criteria (e.g. depth, scope, availability) and external factors (e.g. priorities, time constraints, stage of research, relationship with author) and Tombros, Ruthven and Jose (2005) mix together abstract document characteristics (quality, authority), with physical characteristics (number of links, amount of text).

A serious limitation of these studies is that they seldom identify dependencies among the criteria or condition under which certain criteria may become more or less important to users. In a comparison of results across different search situations, Barry \& Schamber (1998) concluded that "different criteria mentioned under contrasting conditions seem to provide evidence for the 
existence of a few criteria categories that do respond to situational factors...” (p. 234); however, they did not explore these context-dependent patterns further.

Table 2-1: Summary of selected studies of relevance criteria and cues

\begin{tabular}{|c|c|c|c|c|}
\hline Schamber 1991 & Park 1993 & Barry 1994 & Wang 1994 & $\begin{array}{l}\text { Tombros, Ruthven, } \\
\text { Jose, } 2005\end{array}$ \\
\hline \multicolumn{5}{|l|}{ Methods } \\
\hline $\begin{array}{l}\text { User Tasks, } \\
\text { Interviews, } \\
\text { content analysis }\end{array}$ & $\begin{array}{l}\text { User Tasks } \\
\text { Interviews, } \\
\text { content analysis }\end{array}$ & $\begin{array}{l}\text { User tasks, } \\
\text { Interviews and } \\
\text { content analysis }\end{array}$ & $\begin{array}{l}\text { User tasks; } \\
\text { think aloud, } \\
\text { content analysis }\end{array}$ & $\begin{array}{l}\text { Assigned task } \\
\text { situations, Think } \\
\text { aloud, content } \\
\text { analysis }\end{array}$ \\
\hline \multicolumn{5}{|l|}{ Domain } \\
\hline $\begin{array}{l}\text { Occupational - } \\
\text { weather data }\end{array}$ & $\begin{array}{l}\text { Academic } \\
\text { research }\end{array}$ & $\begin{array}{l}\text { Academic } \\
\text { Research }\end{array}$ & $\begin{array}{l}\text { Academic } \\
\text { Research }\end{array}$ & Web searching \\
\hline \multicolumn{5}{|c|}{ Summary of Criteria Identified } \\
\hline $\begin{array}{l}\text { Accuracy } \\
\text { currency } \\
\text { specificity } \\
\text { geographic area } \\
\text { reliability } \\
\text { accessibility } \\
\text { verifiability } \\
\text { clarity } \\
\text { dynamism } \\
\text { presentation } \\
\text { quality }\end{array}$ & $\begin{array}{l}\text { Internal Context } \\
\text { experience } \\
\text { expertise } \\
\text { research } \\
\text { experience } \\
\text { training } \\
\text { External context } \\
\text { search quality } \\
\text { search purpose } \\
\text { availability } \\
\text { priorities } \\
\text { stage of research } \\
\text { end product } \\
\text { Problem Context } \\
\text { definitions } \\
\text { background } \\
\text { methods }\end{array}$ & $\begin{array}{l}\text { Document } \\
\text { depth/scope } \\
\text { validity } \\
\text { tangibility } \\
\text { effectiveness } \\
\text { clarity } \\
\text { recency } \\
\text { obtainability } \\
\text { cost } \\
\text { source } \\
\text { quality } \\
\text { reputation } \\
\text { User } \\
\text { background } \\
\text { ability to } \\
\text { understand } \\
\text { novelty } \\
\text { subjective } \\
\text { accuracy } \\
\text { affectiveness } \\
\text { time constraints } \\
\text { relationship with } \\
\text { author } \\
\text { Other sources } \\
\text { consensus } \\
\text { external } \\
\text { verification } \\
\text { availability }\end{array}$ & $\begin{array}{l}\text { topicality } \\
\text { orientation } \\
\text { discipline } \\
\text { novelty } \\
\text { expected quality } \\
\text { recency } \\
\text { availability } \\
\text { special requisite } \\
\text { authority } \\
\text { relation/origin }\end{array}$ & $\begin{array}{l}\text { Text Content } \\
\text { content } \\
\text { numbers } \\
\text { titles } \\
\text { query terms } \\
\text { amount of text } \\
\text { Structure } \\
\text { layout } \\
\text { links } \\
\text { Quality } \\
\text { scope/depth } \\
\text { authority } \\
\text { recency } \\
\text { general quality } \\
\text { novelty } \\
\text { pictures } \\
\text { physical properties } \\
\text { file errors } \\
\text { language, connection } \\
\text { speed } \\
\text { subscription }\end{array}$ \\
\hline
\end{tabular}

Most of the older relevance studies have been exploratory and qualitative in nature; they were neither looking for evidence of specific dependencies, nor did they collect enough data to show that they exist. Some more recent studies have begun to do so. Tombros and colleagues (2005) studied the impact of two situational variables on relevance criteria: task type and task stage. The 
study involved 24 participants, each searching for three controlled tasks on the Internet, using their preferred method of searching. Three task types were used: background information, decision making, and collecting items, and assessments were made at the start and end of each task to capture the effect of task stage. Despite the preliminary nature of its findings, given the small number of tasks and searchers involved, the study points to variations between the features and criteria used by searchers to assess relevance based on task. A similar experimental websearching study tested a pre-determined set of document features and found that different elements of web pages were used to assess relevance for different types of search tasks (factfinding vs. procedural) (Kelly et al., 2002).

\subsubsection{Summary}

The importance of context in shaping two key aspects of human information behaviour information needs and relevance assessments, suggests that the usefulness of evaluating IR out of context is seriously limited. Some methodological approaches to IR evaluation have been suggested to address this problem. The use of Situated Work Task Situations (SWTS), has been proposed and tested as a substitute for the de-contextualized search topics traditionally used in IR evaluation experiments (Borlund, 2003b). SWTSs provide the searcher with a work task and search context in addition to the search topic, which prompts more natural search behaviour and provides a basis for assessing relevance. SWTSs are now used quite commonly in interactive search studies as a means of providing context and operationalizing differences in contextual variables, such as tasks or domains (Bell \& Ruthven, 2004; Joho \& Jose, 2006; Ramirez \& de Vries, 2006). Reid (2000) went further in proposing the establishment of task-based test collections and evaluation metrics; however, this type of evaluation infrastructure has not yet been adopted. 


\subsection{Workplace Information Seeking and Retrieval}

Workplace information environments have earned the attention of IR researchers due to the rapid growth of networked digital content in recent years. Organizational intranets are complex, heterogeneous information environments that can reach enormous proportions: several years ago the IBM intranet already contained more than 50 million unique URLs on at least 7,000 different hosts (Fagin et al., 2003). However, workplace information collections are known to differ substantially from the Internet. They include a wider range of information sources, such as public websites, intranets, and other electronic documents held in databases, email repositories, and shared directories. Furthermore, they typically contain fewer HTML documents relative to documents in other formats, both structured and unstructured (Hawking, 2004).

The motivation for sharing information within organizations is also different. Whereas Web authors often make an effort to push their documents to their target audience by including special keywords and tags and by linking to related documents, organizational authors tend to be more focused on creating information documents than on marketing them (Fagin et al., 2003). This different approach, together with the relative scarcity of hyperlinks in organizational information spaces, means that Internet search engine technology is less effective within organizations, giving rise to the need for more specialized “enterprise search” applications. One of the challenges associated with developing enterprise search tools is the confidential and proprietary nature of much of the information content, which has meant that it is difficult for researchers to conduct the large-scale evaluation studies typical of system-centred IR research (Hawking, 2004). The TREC Enterprise Track has taken some steps in this direction. To date most efforts in this direction have focused on problems of limited scale, such as expert location and email search within enterprise collections (Craswell, de Vries, \& Soboroff, 2005), but more recent studies have begun to investigate ways to make use of document diversity in terms of genre and quality (Bailey, Agrawal, \& Kumar, 2007; Duan et al., 2007; Shen, Chen, Chen, Liu, \& Cheng, 2007). 
Considerable research has, however, been conducted in the workplace to study general information behaviour and information system use. In particular, a large number of research frameworks have been developed to study professional groups and workplace practices from a contextual perspective. These include information use environments (R. S. Taylor, 1991), communities of practice (E. Davenport, 2002), contextual inquiry (Beyer \& Holzblatt, 1998), situated action (Suchman, 1987), domain analysis (Hjorland, 2002), and cognitive work analysis (Fidel, Pejtersen, Cleal, \& Bruce, 2004; Pejtersen, 1989; Xie, 2005). A common thread running through these approaches is the identification of patterns in work practises and situations as a means of understanding organizational behaviour, improving information practices and/or designing useful information systems. Contextual inquiry, cognitive work analysis and situated action in particular emphasize the importance of studying work practices as they actually occur, through interaction with the employees in their natural settings through the use of mixed methods. These were important guiding principles for this research.

Some general patterns of information behaviour in workplace settings are known. Hawking (2005) noted that the population of workplace information searchers is more homogenous than general search engine users and therefore potentially easier to model. Workplace information searchers are mainly employees who perform known roles characterized by certain sets of responsibilities and skills, associated with certain types of information needs, and their information behaviour tends to be embedded in a task context. Taylor (1991) described information use in professional settings as motivated by the goal of solving work-related problems and as more critical and conscious than general information use. His concept of an information use environment (IUE) is well-suited to describe workplace IS\&R. He described IUEs as settings in which sets of people share common assumptions about their work, deal with common types of problems, deal with typical constraints and opportunities and share assumptions about how problems are resolved. Taylor reviewed studies of various professional groups, and 
identified patterns in types of problems, types of information uses, and in information traits used to resolve the problems. In a relatively rare example of information analysis that is not subjectbased, Taylor and Macmullin (1984) identified multiple dimensions of information in the form of continuums: qualitative to quantitative; hard to soft data; historical to current; single to multiple solutions; factual to diffuse; specific to general; applied to theoretical; operational to descriptive; clinical to aggregated and causal to diagnostic. Building upon the work of Taylor and others ( $\mathrm{T}$. Davenport, 1997), Choo, Detlor and Turnbull (2000b) proposed a behavioural-ecology framework for the design of workplace intranets. The framework includes three nested layers: the information ecology (the organization's social, cultural and political information environment); the information behaviour of actors within the organization, and value-added processes, that "signal, amplify and extend the value of information to the organization and users" (p. 120). The framework suggests that the design of value-added processes in systems, another concept proposed by Robert Taylor (1986), should be based upon analysis of the information ecology and behaviour.

While IUEs represent defined professional or user groups, a generic model has been proposed to represent the type of goal-directed workplace information behaviour described by Taylor (J. D. Johnson, Donohue, Atkin, \& Johnson, 1995). The Comprehensive Model of Information Seeking (CMIS) is analytical and has been tested and generalized based on a number of workplace domains. It considers the impact of two types of variables, antecedents and information carrier factors, on the quantity of information seeking actions. Antecedents are motivating factors, defined as demographics (such as education and search expertise), personal experience (tenure within an organization), beliefs of the searcher about outcomes, and salience (the degree to which it is important to find information). The information carrier characteristics are utility (the degree to which information is directly suited to the needs of the searcher), and information characteristics (such as a document's communication potential and editorial tone). In its emphasis 
on salience, utility and non-topical document characteristics, this model is quite distinct from the standard topical relevance based IR models, and is better suited to workplace IR. However, beyond the identification of interesting variables, the model has little to contribute to the design of IR systems, as it is limited to predicting the quantity of information behaviour, rather than quality or outcomes.

Figure 2-6: Model of the information seeking of professionals (Leckie, Pettigrew, \& Sylvain, 1996)

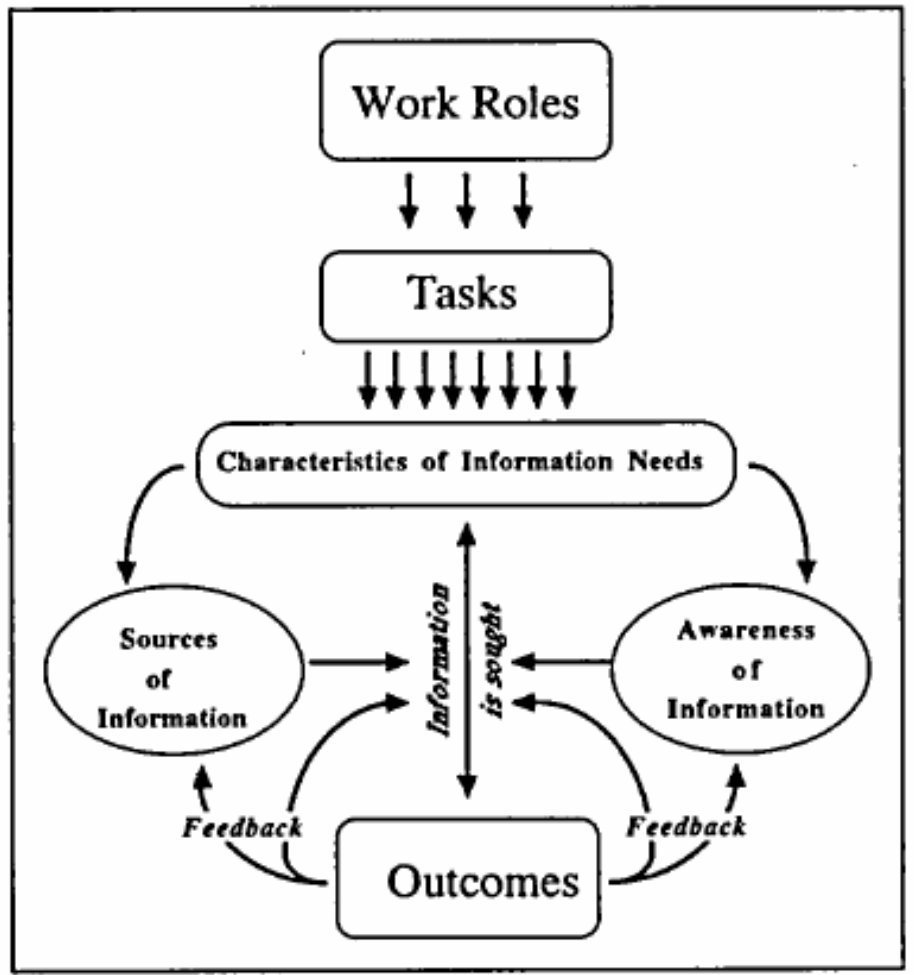

A more specific, but descriptive model of information seeking behaviour of professionals was proposed by Leckie and colleagues (1996) based on a survey of research on engineers, medical professionals and lawyers (Figure 2-6). It emphasizes the impact of six contextual components on information behaviour: work roles and tasks, which drive the information seeking; characteristics of information needs; information sources and awareness of information, both of which mediate the seeking; and outcomes. Some additional intervening variables noted by the authors are: 
demographics (career stage, specialty), project stage, features of the problem situation, and source selection criteria: accessibility, cost, familiarity, packaging, quality, timeliness, trustworthiness. This model provides a useful basis for understanding information behaviour in the research setting, as it includes a large number of variables. It is notable for the emphasis on tasks and roles. However, like most IB models, it is focused more on describing behaviour than on identifying relationships between specific variables.

\subsection{Information Behaviour of Engineers}

There are relatively few studies of the information behaviour of software engineers, the professional domain considered in this research; however, there is a formidable body of research on engineers ${ }^{2}$. As a group, engineers have been characterized as service-oriented professionals with a strong knowledge base in their subject, whose work focuses on completing rather complex tasks and finding solutions to technical problems (Hertzum \& Pejtersen, 2000; Leckie et al., 1996). Studies of engineers have identified some clearly defined patterns of information behaviour. They are known to be highly motivated and heavy consumers of information: estimates suggest that they spend $20 \%-80 \%$ of their work time seeking and manipulating it (Leckie et al., 1996). However, their information seeking is often constrained by time and budget considerations (Fidel \& Green, 2004) such that they typically seek a small amount of "good enough" information rather than comprehensive coverage (Orr, 1970). Engineers tend to rely heavily upon verbal communication with colleagues, internal reports and personal information stores as sources (T. J. Allen, 1977; Hertzum \& Pejtersen, 2000; R. S. Taylor, 1968).

Use of these readily available and easily accessible communication channels and information sources has been interpreted as a manifestation of Zipf's Principle of Least Effort (King, Casto, \& Jones, 1994; Pinelli, Bishop, Barclay, \& Kennedy, 1993); however, recent research suggests that

\footnotetext{
${ }^{2}$ A recent review of this literature (Tenopir and King, 2004) includes a 65 page bibliography of over 1,000 books and articles on the topic.
} 
this principle does not adequately explain how engineers select among information sources (Anderson, Glassman, McAfee, \& Pinelli, 2001). Alternate explanations include: the role of trust (Hertzum, 2002; Hertzum, Anderson, Anderson, \& Hansen, 2002); the challenges associated with finding written information on online environments due to the lack of effective search tools (Hertzum \& Pejtersen, 2000; Kwasitsu, 2003); and the richer opportunities for feedback and contextualization afforded by direct communication (Hertzum \& Pejtersen, 2000). Paepcke (1996) explains how important contextualization can be using an example from an interview study of technical professionals:

To make sense of the material, the engineer had to understand the context of these old designs - the priorities used in decision-making, which designs were rejected and why, and the changes that were made during the design process. This kind of complex, interwoven information is extremely difficult to record... An important step was then to call on the designers who were still with the company to guide him through the material and provide additional context.” (p. 84)

So, while speaking to a colleague indeed may be easier than reading a document, it may also provide more valuable information.

There is further evidence that the "Principle of Least Effort" may not adequately describe the information behaviour of engineers. Accessibility, once thought to be the most important selection criteria for engineers (Leckie et al., 1996; Pinelli et al., 1993), has undergone a reinterpretation. A study of the factors used to select information, based on analysis of 58 interviews with 31 engineers from a range of fields (Fidel \& Green, 2004) found that the majority of selection criteria mentioned (68\%) were, indeed, related to accessibility; however, not primarily in the sense of physical accessibility. Eleven aspects of accessibility were identified, the most important of which were: familiarity with source, efficient to use ("saves time”), physical proximity, format, and level of detail (Fidel \& Green, 2004). Interview participants also mentioned factors related to information quality, content and currency. Studies that focus specifically on software engineers have found that the currency of information is particularly 
important (Hertzum, 2002) as are: content, availability, use of examples, organization, and document type (Forward \& Lethbridge, 2002).

Arguably, some of these criteria, such as format and level of detail, are more strongly associated with utility than accessibility. This is supported by evidence that situational relevance is even more important to engineers than accessibility. In a questionnaire of 872 engineers and scientists, Anderson, Glassman, McAfee, \& Pinelli (2001) tested a number of hypotheses relating to the selection of information carriers. With respect to the selection of written sources (journal articles, conference papers, internal technical reports, etc.), the factors: accessibility, technical quality and prior experience with a source were not correlated with selection, whereas the factor importance to my work was. Although importance to my work was not a well-defined concept in this study, the authors suggest that it may reflect a relationship between the source and its likely contribution to task completion. Similar findings were reported by Hertzum (2002) who found that the key selection factor for software engineers was appropriateness to task and by Fidel and Green (2004) who found that the second most important factor overall was can give data that meets the needs of the project.

Information use among engineers seems to be closely tied to work and task context, and to issues of information access and utility, pointing to the importance of developing more discriminating and context-sensitive search systems for engineers. However, while the literature provides a clear picture of general patterns of behaviour and factors which shape this behaviour, there is very little research that identifies specific dependencies that could be exploited in system design. In addition, there is a need for research that focuses on information behaviour in networked digital information environments, as much of the work on engineers studies more traditional information environments. 


\subsection{Tasks}

The importance of tasks in IS\&R in general and specifically with respect to the engineering and other professional domains has already emerged as a theme in this chapter. Tasks are included as elements of context in numerous conceptual frameworks (Ingwersen \& Järvelin, 2005; Leckie et al., 1996; Mizzaro, 1998; R. S. Taylor, 1991) and are strongly associated with situational relevance and utility. Proposals to re-conceptualize IR as a task-based activity have been made by Järvelin (1986), Vakkari (2003) and Reid (2000), and this is an important component in the contextual approach to IR followed in this research.

A task can be described as "a piece of activity to be done in order to achieve a goal” (Vakkari, 2003); however, in terms of IS\&R, it is useful to distinguish between work tasks versus information seeking and searching tasks (Byström \& Hansen, 2005; Vakkari, 2001). Both these types of tasks play a role in this research.

\subsubsection{Work Tasks}

Work tasks are particular cases of tasks, in which the doer is assigned an activity or unit of work that needs to be completed in order to meet a pre-defined goal, usually having some extrinsic value. When work tasks cannot be completed due to a lack of information, information seeking and search tasks are prompted (Belkin et al., 1982a; Järvelin, 1986). Thus, the work task provides the "embedding task" (Pirolli, 2007), or the environment in which search tasks are conducted and which establishes the criteria for evaluating outcomes. Although this research focuses explicitly on work tasks in a workplace context, it is possible to extend the concept of work tasks to include purposeful leisure activities (Ingwersen \& Järvelin, 2005).

Work tasks have been analyzed from multiple perspectives and at varying levels of granularity. Numerous attempts at task description and classification have identified a large number of characteristics and features of work tasks that may be associated with information behaviour 
(Hert \& Marchionini, 1997; Kim \& Soergel, 2005; Li, 2004). Among these, some of the most commonly studied characteristics are complexity and stage.

The relationship between task complexity and information behaviour (selection of sources and types of information) was studied in a work environment by Byström and Järvelin (2002; 1995). They classified tasks in five groups, according to a priori determinability, ranging from the least complex: automatic information processing, to the most complex: genuine decision tasks. Using interviews and self-recorded journals they collected data from municipal administrators on reallife tasks performed in the course of their work. They found that as task complexity increases, the complexity of the information need increases, the need for domain and problem-solving information increases, more internal sources are used, and the number of sources in general increases. This work extends prior work on task complexity, which found that oral communication is more frequent in more complex projects (Tushman, 1978) and that the number of sources consulted increases with the complexity and duration of tasks (Tiamiyu, 1992).

Vakkari (2001) focused on variations in information behaviour across the stages of task performance. This work extends Kuhlthau’s (1993) Information Searching Process model to identify changes in search terms and tactics at different task stages. Vakkari identified the stages of task performance: task construction (understanding), task performance (strategy \& execution), and task completion (evaluation \& use), and studied the effect of task stage in an academic context of students writing research papers. One important result of this study was the finding that as work tasks proceed, searchers use more and varied search terms to express their topic, and that they increase their search tactics and judge fewer documents to be relevant as their domain knowledge and understanding of their topic grows (Vakkari, 2000).

Another approach to task has been to identify different types of tasks associated with a particular workplace or domain. Algon (1999) used multiple methods to derive and test a task typology for 
project-related work within an organization consisting of seven types: administrative, communicative, information manipulation, document generation, analytic, strategic and operational. The goal of the study was to determine whether information behaviour was dependent upon task, and to determine the role of other intervening variables. A cross tabulation of frequencies of different tasks types and sub-steps against different types of information behaviours indicated that information behaviour was dependent upon tasks.

Some additional studies of professional work settings suggest that variation in task types affects various aspects of information behaviour. A study of city planners identified two core professional tasks - planning and surveying, and a set of supportive tasks - management, support, counselling, training and environmental scanning (Serola, 2006) Data was collected through interviews and Internet search logs, and findings indicate that information channel selection (Internet, intranet, print documents, people etc.) depends upon the type of task and the type of information need. A domain analysis conducted in a pharmaceutical setting used a framework of five work task groupings (basic research, clinical and non clinical tests, marketing and sales, competitive intelligence, environment and safety) to study information behaviour (Lykke Nielsen, 2001). Data was collected using interviews, content and discourse analysis and word association tests, and researchers found significant variation across the five groups with respect to terminology, types of problems and information need characteristics. The findings were applied to the design of a corporate search thesaurus.

More than twenty years ago, Järvelin (1986) wrote: “we argue strongly that ...different types of information have different types of relevance to, and impacts on, different types of work”. A growing body of research focuses on this relationship between work tasks and search behaviour suggests that this is likely to be true, but has produced very little concrete evidence of specific relationships (Hansen, 2005). Studies that focus on generic task characteristics such as complexity or stage are valuable in that findings are likely to be valid in diverse information 
settings. Some implications of these studies with respect to IR systems are that for more complex tasks, searchers would likely prefer to retrieve a wider range of source types, more internal information and sources with background information about the task domain and the search problem. As they proceed with their task and their knowledge of the topic grows, they may prefer sources with more topical depth and less breadth. In contrast, findings from studies of domainspecific work tasks are not as definitive and are less generalizable; however, they may be of more value in designing search tools and systems for specific work settings.

\subsubsection{Information Tasks}

Embedded within the work task is another layer of context for the search situation: information seeking and searching tasks. Like work tasks, information tasks have been defined and classified in a number of different ways; there is no agreed-upon nomenclature or typology. Allen’s 1997 book entitled Information Tasks refers to cognitive activities that take place in the course of interacting with information: scanning, evaluating, learning and planning (B. Allen, 1996). Other common types of information tasks (often called search tasks) used in studies of search behaviour are based upon the type of information sought: known item searches, subject or topical searches, fact finding, and question-answering (Hsieh-Yee, 2001).

In this research, information task types are defined based on the user's intended use of the information: learning, fact-finding, making a decision, solving a problem, and doing something. This is similar to the principle of division for Taylor’s (1991) typology of information uses ${ }^{3}$. Using a similar approach, Limberg (1999) compared the research behaviour of students with different kinds of information goals (fact-finding, assessing an issue and reaching a decision, understanding a topic) and found that they handled and evaluated information differently with respect to a number of parameters, including relevance, bias, information quantity and authority.

\footnotetext{
${ }^{3}$ Taylor identified eight categories of information use: enlightenment, problem understanding, instrumental, factual, confirmational, projective, motivational, and personal or political.
} 
Some recent work has examined the effect of information task variation on search behaviour, and specifically, on relevance assessments (Kelly et al., 2002; Tombros et al., 2005). As noted in section 2.3.2 above, these studies found that the features and elements of pages used to assess relevance were dependent upon information task type.

So, there is strong evidence that both work tasks and the embedded information tasks influence searching and selecting behaviour. However, in an extensive review of the literature on taskbased information seeking, Vakkari (2003) noted that "our understandings of the mechanisms that link characteristics of tasks to search activities are both tentative and limited” (p 452). We still have relatively little understanding of what kinds of information are best suited to different types of tasks, and almost no practical applications of task-based variation exist in search systems.

\subsection{Digital Genres}

Genre is a non-topical characteristic of documents that has received very little attention in IR research, although Ingwersen and Järvelin (2005) mention genre as a contextual variable of interest in the IR interaction. The concept of literary genre has been used as a means of classifying texts since ancient times; however a functional approach to the identification of genre has developed in the past several decades, which views genre as a communicative act (Bazerman, Little, \& Chavkin, 2003). In this sense, genres are "typified communicative actions characterized by similar substance and form and taken in response to recurrent situations” (Yates \& Orlikowski, 1992, p. 299). Genre types are prevalent in organizational settings, and functional genre theory has been used to study communication in organizational settings (Yates \& Orlikowski, 1992). Genre analysis is used also as a method to make sense of organizational behaviour and information systems (Firth, 2003; Honkaranta, 2003; Spinuzzi, 2003).

Genre types are identified based on some combination of form, content and/or purpose features of documents. Form can be further distilled to medium, structure and linguistic features (Orlikowski 
\& Yates, 1994). More recent work focusing specifically on digital genre has included mode of interaction as an aspect of form (Askehave \& Nielsen, 2005; Shepherd \& Watters, 1999). However, genres are not simply document or artefact types based on a set of features. Rather, they are expressions of a tradition: "they emerge from cultural-historical activity and represent, reflect, stabilize, and help constitute the activity” (Spinuzzi, 2003, p. 41). Because they are socially-constructed within particular communities, genres serve as carriers of context; they represent elements of the "who, what, where, when, why and how" of a document, which makes them particularly useful when people are trying to apply information in a real-world situation (Yoshioka, Herman, Yates, \& Orlikowski, 2001).

There is a clear connection between domain and genre, in that "different disciplines or discourse communities develop special kinds of documents as adaptations to their special needs” (Hjorland, 2002). The sets of genres in common usage within particular domains or communities of practice are known as genre ecologies (Spinuzzi, 2003) or genre repertoires (Orlikowski \& Yates, 1994). Genre repertoires serve to promote mutual understanding and make documents recognizable among members of groups (Orlikowski \& Yates, 1994). Genre is particularly valuable in this regard, as it provides visual cues in the "physical landscape of a document”; these cues contain “distinctive, salient features that inform users about a document's identity”(Toms, 2001). While a specific genre category carries contextual information with respect to a particular information object, the genre repertoire as a whole functions as context for IS\&R activities in certain domains.

Recently, genre has been recognized as a possible means of supporting more targeted IR through automatic identification of genres on the Web (Karlgren, 1999; Rosso, 2005a; Roussinov et al., 2001; Shepherd, Watters, \& Kennedy, 2004). An early genre analysis of a sample of 837 web pages found 48 different genres represented (Crowston \& Williams, 2000). In a separate study, researchers conducted interviews with 184 participants who were using the Internet to search for their own needs, in order to determine whether there is a relationship between the purpose of a 
search and the genre in which information is retrieved. Prior to the search, participants were asked to describe their problem and to anticipate potentially useful genres. Web pages actually retrieved from the Internet were collected and these were assessed by participants who characterized their genre as well as their relevance and utility. Researchers also classified the genres of the 1,234 web pages retrieved, identifying 116 different genres within the set of all retrieved documents. Of the set, 158 were not classified, and the agreement in classification between the researchers and participants was less than 50 per cent (Roussinov et al., 2001). The study found a positive relationship between search type and genre, with certain sets of genres tending to be chosen as relevant for particular types of searches. Rosso (2005b) was interested in establishing a set of well-recognized genres to support web retrieval in the education domain. Using card sorting and naming studies, he identified a taxonomy of 18 commonly recognized genre categories and labels. Further testing in which participants were asked to classify a set of documents according to the taxonomy achieved over $70 \%$ agreement among study participants, suggesting that usable taxonomies of web genres can be created.

Genre is a recognized feature of both organizational information spaces and the Internet, and there is evidence that the relevance of types of genres varies depending on the type or intent of a search. Rosso’s work suggests that relatively small sets of well-recognized genres can be identified even in the diverse community of Internet users. Within more homogenous organizational settings, this should be easier.

Practical applications of genre in search systems are rare. Some experimental systems have been proposed or developed (Bretan, Dewe, Hallberg, Wolkert, \& Karlgren, 1998; Glover, Lawrence, Gordon, Birmingham, \& Giles, 2001; Roussinov \& Chen, 2001), but in most cases these systems are based on filtering or categorizing search results by genre, which assumes that searchers are able to specify their preferred genres while searching. This is a tenuous assumption given what is known about the anomalous state of human information needs. The IR research community has 
begun to focus attention on the relationships between genre and search task types through the High Accuracy Retrieval of Documents (HARD) track of the TREC, and some interesting work is starting to be done in this area, which may find its way into future search systems (Belkin, Muresan, \& Zhang, 2004; He \& Demner-Fushman, 2003).

Two associated challenges in working with genre are complexity and scale. In any given information environment there can be hundreds or thousands of different categories and subcategories of genres that are recognized collectively by the members of the user community, so there is a need for practical genre analysis tools to develop effective taxonomies efficiently (Tyrväinen \& Päivärinta, 1999). Furthermore, manual genre classification through meta-tagging or other methods is not a practical solution due to the size and dynamism of digital collections. In order to make use of genre at the organizational level or in Internet search environments, robust automatic classification methods are needed. Machine learning approaches are well established for subject classification and have shown some promising results for genre classification as well (Kushmerick \& Finn, 2006; Lee \& Myaeng, 2004). Work in genre classification is in early stages, but rapid progress is expected due to high interest and activity in this area (Santini, 2006).

\subsection{Summary}

In this chapter I have described the background and motivation behind this dissertation research. There is a need to extend IR models to include aspects of the context in which IS\&R activities take place. Greater context is needed because IS\&R behaviour is complex, dynamic and heavily dependent upon contextual factors and situations. These contextual factors establish a framework of motivations, constraints, goals and criteria within which IS\&R occurs, and as human information intermediaries have long known, understanding this framework is the key to satisfying the often amorphous and poorly articulated information needs of searchers. 
Although context is a very broad concept, some consensus is forming around the contextual elements that play a major role in shaping IS\&R activities: socio-cultural and physical settings, human actors, systems, information objects, the interaction processes over time, tasks, problems and goals. At a more granular level, there is still little agreement as to the key variables within each element, which has meant that very little coordination or cohesion exists in research efforts to date. Furthermore, few of the theoretical frameworks that define contextual variables have been operationalized and empirically tested, and few of the empirical studies reference a particular theoretical framework.

There is a particularly strong association between context and information behaviour in the workplace, as evidenced by the numerous contextual methods developed to study work practices. IS\&R activities in the workplace are embedded in work tasks, and for engineers in particular, their work serves as a key point of reference when evaluating information sources. Various studies have shown that both work tasks and information tasks influence searching and selecting behaviour in various ways, and that different characteristics and types of tasks are associated with particular characteristics of information objects. However, there is no consensus as to the nature of the relationship between tasks and information characteristics, and very few clear and specific conclusions can be drawn from this body of work. Genre is one feature of information objects that has received some attention from the IR research community, primarily as a means of filtering and classifying search results. However, despite the fact that functional genre theory clearly posits a relationship between activities, goals and organizational genres, the relationship between tasks and genres has not yet been tested or employed in a systems setting to date.

In this dissertation, I address a number of the general issues identified in this chapter, by exploring, analyzing and testing relationships between IS\&R activities and contextual variables in the engineering domain. Results will help to fill in some of the gaps identified between general models and specific relationships in information behaviour, and between theory and practice in 
contextual IR. More specifically, this research will contribute to our understanding of the role of task and genres and their relationship in workplace information environments. 


\section{Research Design}

\subsection{Overview}

With the aim of taking a situated and pragmatic approach to workplace searching, this research was conducted in-situ, within a real-world workplace setting and a well-defined professional domain. It used a multi-phase, mixed methods design, starting from an exploratory phase to characterize the role of context in the domain and identify the main contextual factors: those aspects of the broader environment of the search that function as independent variables and play a role in shaping the search interaction. It then moved to a more focused examination of selected contextual factors and their implementation as a filtering component to a search system. Because of the complexity of the design and the effect of the research setting on the design, I first describe the research site, the study population and their information environment. I then provide an overview of the multi-phase research design including the research questions addressed at each phase, the variables tested and the methods employed. More detailed descriptions of the methods used in the different phases of the study are described in the relevant chapters to follow.

\subsection{Study domain and setting}

The setting chosen for this study was the services division of a large multi-national high-tech company, among a population of software engineers working as software services consultants. The domain (software engineering), corporate setting and target population were self-selected to some extent, in that service managers from the company approached our research group seeking 
help with the challenges faced by their employees in locating the information they need to work effectively. At the same time, the extensive literature available on the information behaviour of engineers indicated that this population would be well-suited to a study of contextual search, as engineers tend to be strongly motivated and heavy consumers of information with wellestablished information seeking and use practices (Leckie et al., 1996). The research was conducted collaboratively through a joint academic-industry research initiative, which afforded relatively open access to the study population and their workplace information environment over a number of years.

The study population represents a sub-group within this particular enterprise environment. Software services consultants in this company come from a range of educational backgrounds, but the core disciplines are computer science and software engineering. Their work activities are centred on providing expert assistance and guidance to industrial and commercial clients in implementing software systems and solving technical problems. As consultants, they perform a wide range of software engineering tasks and are required to have expertise in a large and dynamic range of technologies and products. They are reliant upon information to do their work, and are heavy users of information in digital formats.

The software services division of this company employs thousands of individuals world-wide and is divided into groups according to the company's main product lines. Although services consultants perform similar types of tasks company-wide, different product groups use significantly different information sources. Service consultants from two product groups participated in the initial phase so that findings would not be overly-specific to a particular product group, but in the later phases, consultants from a single product group with about 500 members working across North America were the focus. About 20 consultants from this group worked out of the local office where most of the research was conducted, but samples for the various studies were not restricted to this local group. 
The information environment of the research site and population is characteristic of many large organizations. It is very large, distributed, contains heterogeneous types of information and data, is crisscrossed by organizational partitions and contains a rich store of information related to the technologies and products in its purview. Broadly divided, it consists of the Internet, including the organization's public website; an enterprise-level intranet; internal organizational and team databases; and the information stored locally on the personal computer of each employee. The complexity of this information environment makes it a good candidate for a contextual search system capable of serving the needs of specific sub-domains of user populations.

In addition to the advantages of being able to do this research on site in a corporate workplace environment, there were associated challenges. The corporate high-tech world has certain priorities and expectations that make the accommodation of academic research difficult, and viceversa. So, work with this group was constrained by some aspects of the software engineering domain (Singer \& Lethbridge, 1996), and this particular corporate environment. The work of software consultants is highly specialized and technical and varies greatly from one project to the next. They are often working out of the office at customer sites, under serious time and cost constraints. Since so much of their work is done independently via computer, there is relatively little social interaction involved that can be easily observed by a third party. Finally, much of the information they create and use is confidential and valuable, and cannot be removed from the premises. These constraints made it quite challenging to find ways to study this group, to recruit participants, and to collect data.

\subsection{Research Design Approach}

In drawing upon information behaviour models to inform the design and evaluation of an improved IR system, this research spans the disciplines of library and information science and information retrieval. The interdisciplinary character of this approach raises some 
methodological challenges, which have been the source of friction between these research communities to date. Taking a strict paradigmatic approach to research design, one can argue that the methods employed in a research project should reflect the researcher's metaphysical approach to the world. This is a problematic stance for this research study, which is based on two different and competing assumptions. The first is an explicit assumption about the importance of situated, domain-specific factors in shaping information behaviour. The second is an assumption that patterns of information behaviour are based on contextual factors that exist independently of specific, situated domains - contextual factors that can be identified and utilized to improve the performance of retrieval systems. The first assumption leans towards an interpretive epistemology, in which human actors are portrayed as shaping and constructing their own reality through a complex interaction with their own context. The second is positivistic in its underlying premise that some static and objectively knowable reality exists, which can be identified and quantified.

A pragmatic approach to reconciling these different approaches is to use mixed methods to conduct research. Mixed methods accommodate different types of research questions and data, and offer the potential for deeper understanding of a given problem (Greene \& Caracelli, 1997; R. B. Johnson \& Onwuegbuzie, 2004). The mixed methods approach is commonly used in related research areas, such as human computer interaction (Dillon, 2004) and field studies of the workplace (Hackos \& Redish, 1998; Singer \& Lethbridge, 1996).

The research questions addressed in this study relate to the relationship between context and workplace searching, and thus could be best addressed through field research in a naturalistic setting. The design of the first phase of the study draws upon a range of methods used to study workplace activities in-situ: contextual inquiry (Beyer \& Holzblatt, 1998), cognitive work analysis (Fidel \& Pejtersen, 2004), and domain analysis (Hjorland, 2002; Lykke Nielsen, 2001). However, an accepted method for evaluating IR systems on the basis of utility within specific 
contexts does not yet exist. Fundamentally, the extent to which a system retrieves useful, situationally relevant results can only be judged in a real situation by a real searcher, who is afforded the opportunity not only to assess the retrieved information, but to make use of it. This type of extended field study was not possible within the constraints of this research; however, design of the evaluation phase draws upon proposed frameworks designed to incorporate more realistic settings and goals into controlled experimental studies (Kekäläinen \& Järvelin, 2002) (Borlund, 2003b; Reid, 2000).

\subsection{Research Design}

Broadly stated, this study followed a "sequential exploratory" model, in which the qualitative research primarily served to inform the quantitative study to follow (Creswell, 2003). However, the research involved multiple phases and a number of separate studies, some of which used mixed methods concurrently for purposes of triangulation, some sequentially in order to extend or expand understanding, and others which were distinctly qualitative or quantitative.

The three phases of research are each presented in separate chapters of this dissertation, as follows:

- $\quad$ Phase One - identification of key variables and model development (Chapter Four)

- Phase Two - exploration and validation of task-genre relationships (Chapter Five)

- $\quad$ Phase Three - implementation and evaluation of task-genre relationships in a prototype system (Chapters Six-Seven)

Each phase included two or more separate studies. An overview of the methods used in the various phases and studies is presented in Table 3-1. 
Table 3-1: Overview of research design

\begin{tabular}{|c|c|c|c|}
\hline Source of Data & Nature of Data Collected & Analysis & Objectives \\
\hline \multicolumn{4}{|c|}{ Phase One: Workplace domain study - Identification of Contextual Factors and Development of Model } \\
\hline $\begin{array}{l}\text { Workplace domain } \\
\text { study - Pre-study }\end{array}$ & $\begin{array}{l}\text { informal interviews and } \\
\text { field notes } \\
\text { ranked lists of } \\
\text { information sources, } \\
\text { work tasks, and problems }\end{array}$ & $\begin{array}{l}\text { Informal content } \\
\text { analysis: identification } \\
\text { of themes and concepts }\end{array}$ & $\begin{array}{l}\text { Validate importance of } \\
\text { context in shaping IB in this } \\
\text { domain; preliminary } \\
\text { taxonomies of tasks and } \\
\text { problems; seed list of } \\
\text { information sources used to } \\
\text { develop full resource list }\end{array}$ \\
\hline $\begin{array}{l}\text { Contextual } \\
\text { Interviews }\end{array}$ & $\begin{array}{l}13 \text { interviews } \\
\sim 250 \text { instances of work- } \\
\text { information relations }\end{array}$ & $\begin{array}{l}\text { Content analysis using } \\
\text { emergent coding }\end{array}$ & $\begin{array}{l}\text { Identify key contextual } \\
\text { factors and relations among } \\
\text { them; develop contextual } \\
\text { model; develop taxonomies } \\
\text { of work tasks and } \\
\text { information goals }\end{array}$ \\
\hline \multicolumn{4}{|c|}{ Phase Two: Exploration and Validation of Task-Genre Relationships } \\
\hline Metadata Analysis & $\begin{array}{l}\text { Document repository } \\
\text { with } 5800 \text { document } \\
\text { records marked with task } \\
\text { and genre metatags }\end{array}$ & $\begin{array}{l}\text { Cross tabulation and } \\
\text { Correspondence } \\
\text { analysis }\end{array}$ & $\begin{array}{l}\text { Identify underlying sources } \\
\text { of task-genre relationships; } \\
\text { identify similar tasks and } \\
\text { genres and Task -Genre } \\
\text { pairs }\end{array}$ \\
\hline $\begin{array}{l}\text { Taxonomy } \\
\text { Development }\end{array}$ & $\begin{array}{l}\text { Evidence of task and } \\
\text { genre categories in use in } \\
\text { the domain, interviews, } \\
\text { document analysis }\end{array}$ & $\begin{array}{l}\text { Comparison and } \\
\text { manual classification }\end{array}$ & $\begin{array}{l}\text { Develop representative sets } \\
\text { of defined tasks and genres }\end{array}$ \\
\hline $\begin{array}{l}\text { Online Validation } \\
\text { Questionnaire }\end{array}$ & $\begin{array}{l}\text { Ratings of all genres for } \\
\text { each information task; } \\
\text { ratings of each work task } \\
\text { with respect to eight task } \\
\text { characteristics }\end{array}$ & $\begin{array}{l}\text { Descriptive and } \\
\text { analytical statistics: } \\
\text { ANOVA, correlations. }\end{array}$ & $\begin{array}{l}\text { Identify work task types, } \\
\text { Develop Task Genre } \\
\text { Association Matrix }\end{array}$ \\
\hline \multicolumn{4}{|c|}{ Phase Three: Implementation and Evaluation of Task-Genre Relationships } \\
\hline $\begin{array}{l}\text { Experimental User } \\
\text { Study }\end{array}$ & $\begin{array}{l}\text { Search outcome metrics: } \\
\text { efficiency, effectiveness } \\
\text { and satisfaction for two } \\
\text { versions of system; } \\
\text { qualitative feedback on } \\
\text { system and features used } \\
\text { to determine usefulness }\end{array}$ & $\begin{array}{l}\text { Descriptive and } \\
\text { analytical statistics: } \\
\text { ANOVA }\end{array}$ & $\begin{array}{l}\text { Evaluate the effect of } \\
\text { implementing Task-Genre } \\
\text { relationships on search } \\
\text { system usability }\end{array}$ \\
\hline $\begin{array}{l}\text { Retrieval Precision } \\
\text { Study }\end{array}$ & $\begin{array}{l}\text { Retrieval precision at } 5 \text {, } \\
10,15 \text { and } 20 \text { results. }\end{array}$ & $\begin{array}{l}\text { Mean Average } \\
\text { Precision }\end{array}$ & $\begin{array}{l}\text { Evaluate the effect of } \\
\text { implementing Task-Genre } \\
\text { relationships on retrieval } \\
\text { precision }\end{array}$ \\
\hline
\end{tabular}

\subsubsection{Phase I: Workplace domain study}

This phase of the project was designed to identify the key contextual factors that shape

information searching and selecting behaviour in this domain, and to develop a domain-specific

model of the relationship between these contextual factors and information behaviours. 


\section{i. Workplace Domain Pre-Study}

An exploratory study was designed to learn more about work practices and information access issues in the study setting in order to determine the suitability of a contextual approach and to identify potential issues and approaches to guide the design of the remainder of the research study. It was conducted through weekly visits to the research site over the course of a number of months. A range of qualitative data collection methods were used to interact with the study population, including a focus group, informal interviews and job shadowing.

\section{ii. Contextual Interviews}

Following the workplace pre-study, a series of semi-structured contextual interviews were conducted with members of the study population. The questions guiding this study were derived from the first general research objective: To identify and model the role of context in shaping the searching and selecting behaviour of software engineers in a workplace domain.

The research questions addressed in this phase were:

Research Question 1: Which contextual factors are important in determining how software engineers search for information and select documents?

Research Question 2: What relationships exist between contextual factors of searchers at work and their information searching and selecting behaviour?

Semi-structured interviews were conducted in the workplace in order to elicit a user-centred and situated perspective on information seeking and searching, and to allow for some flexibility in the scope of inquiry at this early stage. Interviews were designed using a contextual model of information seeking, following approaches used in cognitive work analysis and information seeking research (Fidel \& Pejtersen, 2004; Kari \& Savolainen, 2003). Qualitative analysis of interview transcripts focused on the identification of themes, categories of tasks, information 
problems and sources, and correlations between work practices and information behaviour.

Findings were used to identify key contextual factors and to develop a model of relationships between contextual factors and information seeking and searching in this domain. Evidence of a relationship between user tasks and document genres emerged from this analysis.

\subsubsection{Exploration and Validation of Task-Genre Relationships}

This phase of the project focused on the validation and further exploration of relationships between the work tasks, information tasks and genres identified in the previous phase as key contextual factors. The specific research questions guiding these studies were derived from the second general research objective: to identify, characterize and operationalize a subset of interrelated contextual factors that have a significant influence on searching and selecting behaviour.

The research questions addressed in this phase of the study were:

Research Question 3: What are the main categories and characteristics of tasks and of genres in this domain?

Research Question 4: Does a significant relationship exist between task and genre in this domain?

Research Question 5: Are there patterns of association among the categories of these variables that can be identified and exploited for IR?

Three separate studies were conducted to address these questions.

\section{i. Metadata Analysis}

To test for a significant relationship between task and genre, and to further explore the relationship between these two contextual factors, a study of a large set of meta-data in a document repository was conducted. Cross tabulation and correspondence analysis techniques 
were used to analyze and explore the associations between task and genre meta-tags in this repository.

\section{ii. Taxonomy Development}

In order to identify the types of tasks and genres prevalent in this information environment, data on tasks and genres was collected during the contextual interviews as well as through a genre analysis of the information environment. Based on the findings, taxonomies in the form of simple lists and definitions of prevalent tasks and genres, were developed.

\section{iii. Validation Survey}

An online questionnaire was conducted to validate the relationship between tasks and genres and to collect some additional data using a larger sample of the study population. The questionnaire was designed to collect data on the specific relationships between genres and information tasks, as well as to identify broader characteristics of work tasks in this domain. Data from this study was used to inform the design of the system and the research methods used in the evaluation study to follow.

\subsubsection{Implementation and Evaluation of Task-Genre Relationships}

This phase of the research was guided by the third general objective: To evaluate the contribution of incorporating contextual factors into a search system. The goal of this phase of research was to test the use of task-genre relationships in a search system. The components of the search system that made use of task-genre relationships were designed based on the findings from the first two phases of the study.

The research question addressed in this phase was:

Research Question 6: Does the use of task-genre relationships to filter search results improve search outcomes? 


\section{i. Experimental User Study}

An experimental user study was used to evaluate search outcomes for two versions of a prototype search system: an experimental system using filtering based on task-genre relationships and a Baseline system with basic search engine functionality. The general hypothesis tested in this evaluation was that the contextual system would provide more efficient access to more useful documents than the Baseline system. A within-subjects experimental design was employed, in which each of 32 participants searched for four of a total of eight tasks, two with each version of the system. Measures included a range of search outcome metrics representing efficiency, effectiveness and satisfaction, as well as some additional measures of search behaviour. Qualitative data was collected with respect to the selection criteria used to identify relevant documents, and feedback on the search system.

\section{ii. Retrieval Precision Study}

In order to compare retrieval precision between the Experimental and Baseline systems, a small test collection was created using the search tasks, relevance judgments and queries from the user study. Eighty user formulated queries (10 per task) of varying lengths were run against the Baseline and Experimental systems. The first 20 results from each run were collected and mean average precision across all queries was calculated at 5, 10, 15 and 20 results for each system and each search task.

\subsection{Summary}

The software services setting selected for this research is well-suited to an application of contextual search due to the large size and high level of complexity of the information environment and the importance of information to the work of this group. The research was conducted in phases, each dependent upon the findings of the former, leading from initial exploratory phase, to a more focused validation phase, and finally to the implementation and 
empirical evaluation of tasks-genre relationships in a search system. The following chapters describe the methods and findings of each study phase in detail. 


\section{Workplace Domain Study}

\subsection{Overview}

This chapter reports on the initial phase of research, which was conducted in response to the first study objective: to identify and model the role of context in shaping the searching and selecting behaviour of software engineers in this work domain.

A workplace domain study was conducted to gather data on the work practices and information behaviour of the study population. An initial exploratory pre-study was used to understand the role of context within this domain and to develop a framework for the ensuing research. This was followed by a more in-depth study using semi-structured interviews.

The methods and findings of the two parts of the workplace domain study are presented below in separate sections. Outcomes from this phase lead to the identification of the key contextual factors in this domain, as well as a model of the role of contextual factors in shaping information searching and selecting behaviour, which is presented in the final section of the chapter.

\subsection{Pre-Study}

The pre-study was conducted to identify the broad information access challenges faced by this population, with a view to identifying which of the many potential contextual factors are influential in this domain, and to learn more about the study population and the effectiveness of diverse research methods to inform the design of the studies to follow. The pre-study was 
conducted on-site intermittently over a period of several months, affording the opportunity to become familiar with the organizational culture and information environment.

\subsubsection{Method}

Three data collection methods were used:

\section{i. Unstructured interviews}

Five unstructured personal interviews were conducted with members of the target population. Participants ranged from intermediate to senior level consultants with extensive experience in the field. Interviews lasted from 30-60 minutes and focused on the nature of the consultants' work and their information seeking and use habits. Participants described their work, explained how they seek and use information in the course of their work, and identified challenges related to finding and using information. They also recalled and described one or more specific incidents in which they had searched for information. Interviews were not recorded; notes were taken by a second researcher in the room during the interview.

\section{ii. Focus group}

Using electronic meeting software, one on-site focus group was conducted with seven volunteer participants from the software services consulting group. Participants were recruited by contacts within the company. The group met for a one hour lunchtime meeting in a computer lab equipped with GroupSystems software. Using the "group outliner” tool provided by the software, participants collaboratively constructed a list of their main work tasks (Table 4-1), as well as the common information problems and information sources associated with each task. Participants used the "vote" tool to assign scores of high, medium or low importance to the list of all tasks and the list of all information sources. 


\section{iii. Job shadowing}

The study population conducts much of their work off-site, on the premises of clients and customers. In order to observe their information practices while on remote assignments, three days were spent shadowing a senior consultant engaged in troubleshooting and providing technical advise on a long term consulting project for a major client. Data collection included field notes of observations on the working environment and the consultant's interactions with the client and with other consultants, as well as a log of the information problems encountered by the consultant during that time, and the steps taken to resolve them.

\subsubsection{Data Analysis}

Interview notes were used to identify recurring themes, issues and challenges. Data from the focus group was summarized by the GroupSystems software in the form of lists of work activities, information sources and specific problems. Data collected during job shadowing was used to augment these lists.

\subsubsection{Findings}

\section{i. Information practices}

Consultants uniformly expressed their heavy reliance upon information to complete work tasks and resolve problems, but noted that they are often unable to find the information they need. One senior consultant remarked that he is faced with an "ocean of information," and said that he regularly receives “desperate emails” from junior colleagues unable to find answers to their questions. Information use is almost exclusively digital, and draws upon sources located on the corporate intranet, internal company databases, the World Wide Web and personal document collections stored locally on personal laptop computers. A wide variety of tools and techniques are used to find documents: search engines, directories, known URLs, bookmarks, and hypertext links. The focus group identified over 40 specific information sources (websites, repositories, forums, etc.) that are regularly used by this group. 


\section{ii. Work practices}

These consultants are primarily engaged in consulting projects, which vary in length from single day "critical-situations" to multi-year projects. Their work environment changes frequently, as each project involves different clients, technologies, team members, services and activities. In describing projects, consultants tended to categorize them by the different types of work activities or tasks performed, as in "a migration project" or "a troubleshooting project". The focus group identified 21 common work tasks performed by this group, which are listed in Table 4-1.

Table 4-1: Common work tasks identified by focus group*

\begin{tabular}{ll}
\hline 1. Troubleshooting & 12. Proof of concept \\
2. Performance analysis & 13. Software application upgrade \\
3. Consulting and advising & 14. Design or migration workshop \\
4. Software design & 15. PMR submission and tracking \\
5. Migrations from competitor products & 16. Marketing / pre-sales \\
6. Customization & 17. Competitive bake-offs \\
7. Installation and Deployment & 18. Teaching \\
8. Mentoring clients on technologies & 19. Prototyping \\
9. Programming & 20. Testing \\
10. Software compatibility & 21. Site planning workshop \\
11. Firefighting & \\
\hline
\end{tabular}

*In order of decreasing importance, based on participant ratings

\section{iii. Methodological findings}

The pre-study identified some important methodological issues. Consultants' busy schedules and heavy time commitments on the job proved to be major obstacles to recruiting study participants. The approval of managers had to be obtained before approaching consultants, and then consultants had to be willing and able to allocate time in between travel and tight deadlines. Monetary compensation was found to be neither appropriate nor meaningful for this population, as participants were motivated to take part primarily by team and collegial connections and the desire to contribute to a solution to the "information problem".

Job shadowing proved to be a poor method of learning about IS\&R behaviour in this context, since most of this group’s work, including interactions with clients and colleagues is not conducted through face-to-face interactions, but rather, occurs via channels which are difficult to 
observe without being obtrusive: email, listserv, chat and search tools, for example. Furthermore, attempts to log or record the information seeking behaviour of consultants would have been untenable due to the associated confidentiality and security risks to both consultants and clients.

Individual interviews proved to be the most effective means of collecting data about consultants' information seeking and work practices. However, attempts to use a critical incident technique in these early interviews proved to be relatively ineffective without associated activity logs, as consultants had difficulties remembering and describing information seeking incidents in detail. Interviewees tended to remember specific problems and solutions, but often could not remember how they had found the necessary information.

\subsubsection{Pre-Study Summary}

The pre-study indicated that this population and setting is well-suited to serve as a test case for contextual search. The group, whose effectiveness as consultants depends upon being able to find useful information efficiently, makes heavy use of information. Their work practices are clearly defined in the framework of individual projects and they perceive the variation in their own work practices as a function of different tasks and situations. The pre-study identified some of the specific problems, tasks and information sources used by this group, to be further explored in the next stage. Furthermore, the pre-study provided valuable input for the design of the methods and instruments in the phases to follow.

\subsection{Contextual Interviews}

Following the pre-study, a more comprehensive study was designed to respond to the following research questions.

RQ1: Which contextual factors are important in determining how software engineers search for information and select documents? 
RQ2: What relationships exist between contextual factors of the searcher at work and information searching and selecting behaviour?

\subsubsection{Method}

Semi-structured interviews were used to elicit a user-centred and domain-situated perspective on the study questions. Use of semi-structured interviews in the workplace has proven to be an effective method of studying workplace activities as input for system design (Beyer \& Holzblatt, 1998; Paepcke, 1996). Useful for exploratory research, they provide a framework for targeted discussions which are not limited to specific topics. In this case, an open framework for discussion was essential as the pre-study indicated that consultants were comfortable talking about their work, but were not used to thinking explicitly about their information behaviour. The semi-structured framework allowed for the negotiation of joint understandings of interview topics, the accommodation of individual differences between participants, and kept the time demands on participants relatively low. Participants were probed with specific questions in the context of discussions about past projects and common work tasks, in order to prompt them to speak about their information behaviour. Hypothetical information problems were also posed to participants to prompt discussions about their information seeking strategies.

\section{i. Participants and Recruitment}

The participants in this study consisted of 13 software services consultants (12 male and one female), recruited by contacts within the company and given permission by their managers to participate during work time. Some were approached personally and others responded to email notices of the study posted to various workgroup lists. Participants were both geographically distributed and from two product groups. The sample included both junior and senior consultants, as career stage has been identified as a factor in shaping information behaviour (Leckie et al., 1996). The sample was small, as recruiting was extremely difficult due to demanding schedules of consultants. It took eight months to recruit volunteers for this set of interviews. 
Participants had worked as consultants for periods ranging from 2 to 10 years, although some had worked much longer in the software industry in other positions. All held undergraduate degrees, primarily in engineering or computer science, but also in other fields including commerce, systems analysis, and public administration. Four held graduate degrees. Their self-described specialties represent a range of technical skills, as well as software architecture and project management.

\section{ii. Procedure and Instruments}

Individual interviews lasted from one to two hours. Half the interviews were conducted by telephone due to geographic location and time constraints and half were face-to-face. All interviews were digitally audio-recorded. Each interview began with the participant reading a description of the study and procedures and signing the consent form (Appendix 1), followed by the interview. Time was available at the end of the interview for participants to ask questions about the study.

Interviews were structured using a layered contextual approach based on the "onion model” used in cognitive work analysis (Fidel \& Pejtersen, 2004; Kari \& Savolainen, 2003). This approach identifies a large number of contextual layers that are likely to influence how people look for information. This study was focused on the layers of context likely to influence patterns of behaviour within the group due to changes in the situation, rather than between this group and other domains, professions or cultures. Interview questions (Appendix 2) were designed to elicit consultants' information behaviour with respect to the six layers of context described below.

- By domain: the role and importance of information in their work as software consultants.

- $\quad$ By individual: areas of expertise and personal strategies for finding information. 
- By project: consulting projects in which participants were recently involved, and the main information problems and resources used in each.

- By work task: discussion based on a seed list of eight broad tasks derived from the prestudy: design, programming, migration, security, testing, performance tuning, installation and configuration.

- By information task: discussion based on a seed list of six types of information tasks: learning about something, finding out how to do something, finding advice, looking up facts, finding a solution, finding a tool.

- By problem: discussion about finding information for eight information problem scenarios developed from questions asked in online discussion groups relevant to the two product groups.

Two additional sections were included in the interview script, which asked participants to examine examples of documents and describe the features they liked or disliked and to provide feedback on a list of various information sources, websites and databases used by their group. However, due to the significant additional time required to conduct these sections, which added relatively little value to our research, they were excluded from most of the interview sessions.

\section{iii. Data Analysis}

The audio recordings of the interviews were transcribed and analyzed using Qualrus qualitative analysis software. Content was coded at several levels. Twenty high-level codes were assigned to identify responses to all the structured interview questions, using a priori codes. This allowed for the analysis of sets of responses together to identify common concepts and themes and to extract a demographic profile for the sample. The next coding pass employed an open approach to tagging references to information needs, work and information tasks, document features, work 
roles and information sources. Participants made recurring references to twelve information sources, each of which was assigned a unique code. Some of these consisted of websites or databases (i.e. Google, webpages on specific products), while others consisted of broader information channels (i.e. people, intranet).

At this stage, dependencies between some of the codes became apparent. For example, Participant 10 noted: "If ...most of my engagement is a troubleshooting thing, then, of course I use search all over the place”. A third coding pass was then conducted to identify all text segments mentioning relationships between aspects of work context and information characteristics, information sources or search behaviours. The set of interviews contained 245 instances of such relationships. The final level of coding focused on these 245 interview segments, and used an iterative and inductive approach to assign codes to recurring concepts. Specific codes were assigned to describe:

- Categories and characteristics of the work context relating to the searcher, the project, work tasks, information tasks and problems;

- types and characteristics of information objects;

- specific information sources;

- types of information behaviour.

\subsubsection{Results}

Findings from the analysis are presented in the four following sections. The first provides a summary of participants' responses to general questions about their information behaviour and the information access challenges they face. The next section responds to the first research question by identifying the key contextual factors identified in the analysis. The third section 
provides a more detailed description of each of the contextual factors and the role it plays in shaping information searching and selecting behaviour. The final section responds more specifically to the second research question, by identifying and modeling the broad patterns of association between contextual factors and information behaviour.

\section{i. Issues of Information Seeking and Use}

The interviews revealed that access to information is extremely important to this group: "finding the information quickly and finding the right information is absolutely critical in our position” (P06). The range of responses from participants indicates that most of them spend between 20$30 \%$ of their time looking for and consulting information sources. One participant noted that this figure would have been higher if not for time constraints: “it is 30\% not because we don't need more information, but because that is what we can spare. We also need to work....You can never have enough. It's just that you spend 30\% of your time finding just about enough then you have to go" (P07). In addition to the time pressures, participants identified the following problems with finding information in their current environment:

- The overwhelming volume of information and information sources: "the amount of data resources ... are just immense. I mean, I bet you I use probably 1\% of them” (P12).

- The lack of a central repository or search portal: "we have sites all over the world, and you really don't know where to go. You might have your favourite sites, but sometimes it takes hours...” (P10). "If there was one way, if there was one place that I can search that I could get confident results, my life would just be so much easier. But because there isn't, ... we just figure out how to do it, we figure out work-arounds” (P03).

- The instability of these sources: participants noted that these sources change locations and names frequently, and become obsolete very quickly; 
- Difficulties getting targeted results with existing search engines: “A lot of times it's also: How do I phrase this so I don't get 2,000 responses? If I look for certain words, I know I'm going to get thousands of responses and they don't mean anything when I sift through them" (P03).

- The poor quality of some of the information: "So there are lots of bad articles out there. How to say which ones are good is very difficult...there are lots and lots of people out there with incorrect information” (P06).

\section{ii. Contextual Factors of the Searcher at work}

Within this challenging information environment, workplace context plays a major role in shaping the searching and selecting behaviours of this group. Ten key contextual factors emerged from analysis of the interview data. These are presented in Table 4-2, divided into four broader spheres of context: person, project, work task, problem.

The contextual factors are a mixed collection of data types and levels of granularity due to the bottom-up approach of extracting them from the discourse of participants. Some of the factors could be measured on an ordinal scale, for example expertise and familiarity; however, in most cases, participants tended to describe these factors using nominal and descriptive categories. Two project-related factors, system and client characteristics, are included here as meta-factors, since participants clearly identified these as important aspects of any project, but no strong consensus emerged as to which specific variables were most important. 
Table 4-2: Key contextual factors influencing searching and selecting behaviour

\begin{tabular}{|c|c|c|}
\hline Contextual Factor & Definition & Variables \\
\hline \multicolumn{3}{|l|}{ Person } \\
\hline Expertise & $\begin{array}{l}\text { General level of professional skill, experience } \\
\text { and knowledge. }\end{array}$ & ranges from novice to expert \\
\hline Familiarity & $\begin{array}{l}\text { Level of familiarity with the subject matter of a } \\
\text { particular project, task or search. }\end{array}$ & range from low to high \\
\hline Role & $\begin{array}{l}\text { A defined position and set of responsibilities } \\
\text { with respect to a particular project }\end{array}$ & $\begin{array}{l}\text { Project manager } \\
\text { Architect } \\
\text { Technical Specialist }\end{array}$ \\
\hline \multicolumn{3}{|l|}{ Project } \\
\hline Length & $\begin{array}{l}\text { The time period allotted for completion of the } \\
\text { project }\end{array}$ & $\begin{array}{l}\text { ranges from short (one day) } \\
\text { to long (one or more years) }\end{array}$ \\
\hline $\begin{array}{l}\text { Stage of } \\
\text { completion }\end{array}$ & Phases in the project lifecycle & $\begin{array}{l}\text { start-up } \\
\text { main work } \\
\text { wrap-up }\end{array}$ \\
\hline $\begin{array}{l}\text { System } \\
\text { Characteristics }\end{array}$ & $\begin{array}{l}\text { A meta-factor including various aspects of the } \\
\text { technical infrastructure involved in a project }\end{array}$ & $\begin{array}{l}\text { Sub-factors: } \\
\text { Software Product/s } \\
\text { Platform } \\
\text { Operating system } \\
\text { Product origin (in-house or } \\
\text { external) } \\
\text { Maturity of technology }\end{array}$ \\
\hline $\begin{array}{l}\text { Client } \\
\text { Characteristics }\end{array}$ & $\begin{array}{l}\text { A meta-factor including various aspects of the } \\
\text { client served in a project }\end{array}$ & $\begin{array}{l}\text { Sub-factors: } \\
\text { Industry sector } \\
\text { In-house skill level } \\
\text { Importance of client }\end{array}$ \\
\hline \multicolumn{3}{|l|}{ Work Task } \\
\hline Consulting & $\begin{array}{l}\text { Broad task categories related to the type of } \\
\text { service provided to the customer }\end{array}$ & $\begin{array}{l}\text { Project management } \\
\text { Training } \\
\text { Mentoring } \\
\text { Reviewing } \\
\text { Proof of concept } \\
\text { Support } \\
\text { Hands-on } \\
\end{array}$ \\
\hline $\begin{array}{l}\text { Software } \\
\text { Engineering }\end{array}$ & $\begin{array}{l}\text { Broad task categories related to the technical } \\
\text { work performed in a project }\end{array}$ & $\begin{array}{l}\text { Architecture \& design } \\
\text { Implementation } \\
\text { Deployment } \\
\text { Installation \& configuration } \\
\text { Integration } \\
\text { Migration } \\
\text { Performance tuning } \\
\text { Troubleshooting }\end{array}$ \\
\hline \multicolumn{3}{|l|}{ Problem } \\
\hline Information Task & $\begin{array}{l}\text { Broad task categories based on intended uses of } \\
\text { information }\end{array}$ & $\begin{array}{l}\text { Learn about } \\
\text { Make a decision } \\
\text { Find out how-to } \\
\text { Find facts } \\
\text { Solve problem }\end{array}$ \\
\hline
\end{tabular}




\section{iii. Impact of Specific Factors}

This section summarizes evidence from the interviews with respect to the relationship between each of the contextual factors and searching and selecting behaviour, including the specific variables identified, the relationship between the factors and information behaviour and dependencies between factors and variables.

Person - Expertise: The level of expertise of a consultant influences the nature of the work they are assigned, the nature of the information that they seek, and their intended use of the information. Novice consultants tend to be assigned to work with mature technologies that are well-documented, perform more hands-on tasks, and use more procedural information. Novices "go for the product detail right away...They'll go and try to figure out the how-to "; whereas, more senior consultants "tend to understand the why" (P08). Senior consultants tend to work with products that are under development, and with more abstract tasks such as architecture, planning, and design, which require more high-level, conceptual information. They are more likely to make use of situated knowledge in the form of intellectual capital (information created and shared by colleagues), which is not publicly available to their clients and thus is more valuable and difficult to access. They also tend to have fewer accessibility constraints, since they have the necessary permissions and personal contacts to elicit cooperation from experts and high-level people within the company.

Person - Knowledge \& Skills: Both novice and expert consultants frequently encounter tasks, products, and technologies with which they are unfamiliar, due to the pace of change in the software domain. When this happens, they spend more time searching for information, and will make use of general overview documents: "for example if I'm not familiar with performance testing, then if I know the best practices then I can pretty much start right away” (P04). This situation is strongly associated with the information task learning. On the other hand, when 
consultants are on familiar ground, they are more likely to have documents in their personal collections to which they can refer, and will tend to be searching for more low-level information.

Person - Role: The roles that consultants play in the workplace are strongly related to the work tasks they perform and the types of information they use. Typical roles are: project manager, architect, and technical specialist. Project managers are more heavily involved with business processes and more likely to use generic process-oriented documents, document templates, standards and guidelines. Architects use high-level conceptual and planning documents while technical specialists use more low-level product documentation that contains detailed technical and procedural information and specifications.

Project: Consulting projects, or engagements, are a major source of variation in the information behaviour of consultants. As one participant explained: “ there are different types of engagements—-from short-term to long-term, from support and problem-resolution to advice and architectural review. Those are completely different games. So it very much depends...on the engagement, it's a different thing that you do...different types of information” (P07). Several project-based variables were identified by participants.

Project - Length: Project length has a strong impact on consultants' information practices, as it establishes the timeframe for information seeking. Consultants tend to distinguish between the very short “critical situation” type projects (one-two days), the medium range (one-two weeks), and the long term projects that last up to a year or more. Very short projects are often associated with troubleshooting tasks and an increased level of urgency. The time constraints mean that "we're under a certain amount of stress to find that information right on the spot” (P05). For these, consultants will not use lengthy product manuals or training materials, and have no time to try out a hands-on solution. Rather, they will make use of search engines to find specific, detailed solutions and published problem reports, or to call up colleagues or request help through product 
support channels. For the middle range projects, there is more time to consult with colleagues through forums and to use a broader range of information materials. Finally, for very long term projects, consultants will be more likely to invest time reading lengthy manuals, using training materials, and taking online courses.

Project - Stage: Consultants noted that the project stage is associated with different patterns of information use: "there are all kinds of information that you need starting, as the project develops... information about the product, basic things, then skills transfer, but then problem resolution as they start running into problems, then the newest, the latest and greatest.” (P07) The initial period tends to involve more information searching, and is also the time when most background overview reading or learning is done. The next stage focuses more on detailed, procedural information and problem-solving, and the final stage is concerned more with project management tasks, such as the preparation of documentation, presentations and reports.

Project - System: the system parameters provide the framework for the technical work involved in a project, and as such, they are critical in determining the topical relevance of information. The main system parameters are: the software products and versions in use, the platform, and the operating system. There are two non-topical characteristics of products that influence information behaviour: ownership and level of maturity. When seeking information about the company's own products, consultants will tend to rely upon sanctioned, internal product documentation. If there are third-party products involved, their main sources of information are usually external web sites and product pages. A product's maturity level also influences the amount and kinds of information available about it. Established products are likely to be fully documented in formal and official documents that are easy to find. Projects involving new releases, "1.0 projects," tend to be very challenging from an information perspective. In these cases, consultants tend to rely primarily upon informal information obtained from colleagues and hands-on problem solving. 
Project - Client: Participants identified a number of aspects about their clients that bear upon their information behaviour, but among these, no single, critical, independent factor stood out. These aspects included: industry sector, skill level of the client's in-house information technology staff, and the nature of the client's relationship with the consulting company. For example, clients who are strong partners of the company are more likely to be using new product releases and to have better access to expert advice from within the consulting organization. Consultants working on projects with such clients tend to be able to find the information they need more easily through these person-to person connections: "because of the importance of this client we had a lot of executives involved in it and so they greased the wheels to get a lot of people to work this issue.... this one really was an example where the information gathering and sharing did not occur through databases, but more directly through people, in most cases” (P11).

Work Task: Interview questions were designed to elicit discussion about work tasks related to software engineering, based on indications from the pre-study that these were the predominant task types. However, in the course of the interviews, it soon became clear that work tasks related to consulting represent another dimension of this work. Tasks such as customer training, mentoring or reviews were commonly mentioned, often in combination with more technical software engineering tasks. For example, consultants might be engaged in a deployment review or a migration training session. Both dimensions of work tasks influence information behaviour.

Work Task - Consulting: the main consulting tasks are: project management, training, mentoring, reviewing, proof-of-concept, support, and hands-on work. These vary in the amount of information seeking they involve and in the types of information used. In general, the consulting task dimension is associated with generic process-oriented documents, such as roadmaps and templates that provide the consultants with guidelines for performing these types of services. There are differences between tasks that are hands-on and thus primarily technical, and overview tasks, such as reviewing or mentoring, that involve more high-level and generic information. 
Training projects tend to use the same genres that consultants use to self-educate, such as course materials, tutorials and best practices. Technical support services are associated with troubleshooting and tend to use more specific and detailed information, and to rely upon documents available through the support web site.

Work Task - Software Engineering: Technical work tasks are the core professional work of software engineers, and there is considerable variation in the way information is used to support different tasks. A set of work tasks was discussed in the interviews, and findings for each are presented below.

Architecture \& Design: These tasks are related to decisions with respect to the components and functions of computer systems and how they interact. Consultants are involved in designing both software architectures and hardware system configurations. In both cases, they will tend to use information that is at a fairly high level of abstraction. This type of information is found in formal documents such as technical manuals and white papers. These will often include design patterns and best practices for design. Another source used for architecture is specific integrated scenarios that can serve as examples.

Implementation: This task is directed at building a software program, i.e. writing the code. Implementation tends to be a detail-oriented task and is associated with specific technical and language requirements. This task is associated with searching for specific facts and specifications, often from known sources, as well as the use of patterns and best practices. In addition, programmers may search for documents or files that contain re-usable samples of code.

Deployment, Installation \& Configuration: These tasks are directed at the process of making an application available for use, getting it running on a system and adjusting the software settings. These are "straightforward day to day" (P05) tasks that are very strongly associated with lowlevel, detailed product documentation. They are highly procedural tasks that tend to require step- 
by step instructions and guides as well as looking up specific settings and parameters. These tasks are well supported by internal documents and consultants tend to have a clear idea where to find this information.

Integration: Integration deals with the process of making separate applications and devices function together. It tends to be scenario-specific work that requires both detailed product and technical information for multiple technologies. Because there are almost infinite possible integration scenarios, in many cases documentation tailored to a specific scenario does not exist or is difficult to find. Consultants tend to use search engines to find information about the external products involved. This task is often associated with troubleshooting work and consultants tend to use a range of product support and technical sales information that related specifically to technical integration issues. For this type of context-specific task, consultants will tend to seek out information that is grounded in experience, either through colleagues or through knowledge sharing channels. Integration tasks also often involve hands-on trial and error work, rather than use of information sources: "when I have time on my own I would actually go and do it myself right” (P01).

Migration: This task is concerned with moving a system from one application or platform to another. In terms of information practices, migration is similar to integration. It uses both technical and product oriented information and often requires gathering information on competitor products from outside sources. Migration makes heavy use of product documentation, technical specifications, and procedural guides or roadmaps. Because this is a common and welldefined work task, there tend to be course materials and tutorials available for use. Although the process is mapped out in a generic way, each migration is scenario-specific, so consultants are likely to look to colleagues with experience when complications arise. 
Performance Tuning: This task involves testing a system and tuning parameters in order to increase its processing speed, load and reliability. Performance tuning generally involves using a range of tools to test the system, and requires solid technical knowledge. It is a fairly low level task, which involves looking up technical details and learning how to use various software tools. However, it is a relatively complex task, which like debugging and troubleshooting involves problem solving, and consultants will often seek out the advice of experienced colleagues. Technical articles and manuals are often used, as well as the support website for brief problem reports and technical tips to improve performance.

Troubleshooting \& Debugging: This task has to do with identifying and finding the source of a problem with a system, and framing a solution. This is a complex task that draws heavily upon system generated information as well as a range of external information sources.

Troubleshooting is dependent on the system context, so consultants are often seeking information about very specific similar cases. They tend to use problem reports and very detailed technical information from technical support databases. Troubleshooting is strongly associated with use of Internet search engines, which can be very effective because system malfunctions are often associated with specific error strings that can be used as queries. Troubleshooting involves a high level of uncertainty, so it requires the kind of information you "cannot read from a book” (P04). When troubleshooting, consultants make heavy use of internal and external discussion forums to find similar cases and to find people with experience who can provide clues, strategies or insights into problems.

Problem: For any given work task, a consultant may need to deal with a number of problems or questions that prompt information seeking tasks. The main factor that influences searching and selection behaviour in this sphere is the information task associated with the particular search situation. Six types of information tasks were discussed in the interviews. 
Learn about: When the goal is to learn about something, consultants generally turn to more highlevel, conceptual information in formal genres: manuals, courses materials, and technical articles. They are often looking for brief overviews to get a quick introduction to a topic. They will often ask other more knowledgeable consultants to recommend reading materials.

Collect advice: When consultants need to make decisions, they are most likely to turn to colleagues either directly or through forums. This is most common when the problems are related to specific scenarios and the solutions are not documented in more generic information sources. Best practices documents can be useful for this type of information goal.

Find instructions: When the goal is to complete some defined task or process, consultants seek out low-level procedural information. They look for detailed step-by-step instructions in product documentation and technical articles.

Find facts: When the goal is to find factual information, such as technical specifications or product settings and features, consultants tend to either go to a known document or to look for an authoritative source through product documentation, internal product web sites, or in external third-party web sites.

Solve Problem: This goal is most strongly associated with the troubleshooting work task, but may also occur in the context of other work tasks. Problem solving tends to be a relatively complex task and involves the use of multiple types of resources. Consultants are often seeking hints and clues and do not have a clear idea what is or is not relevant. They make heavy use of colleagues and technical support documents as sources of information.

Find examples to reuse: When the goal is to find information to reuse, either as content or for a template, consultants often turn to internal databases that contain materials from past projects: reports, schedules, source code, etc. 


\section{iv. Relationships between Contextual Factors and Information Behaviour}

The previous section identified the role of each of the contextual factors and the nature of contextual effects on IS\&R behaviour. This section presents a high-level analytical summary of the factors and relationships described above. These findings are summarized in the form of a model, presented in Figure 4-1. On the left of the diagram, the contextual factors described in Table 4-2 are represented by the categories: searcher, project, work task and problem. The other three components in the diagram represent information behaviour: accessibility constraints, relevance criteria and information seeking strategies. Five general types of relationships between the components were identified, and are described below.

Figure 4-1: Model of Contextual Effects on Searching and Selecting Behaviour

\section{Workplace Information Environment}

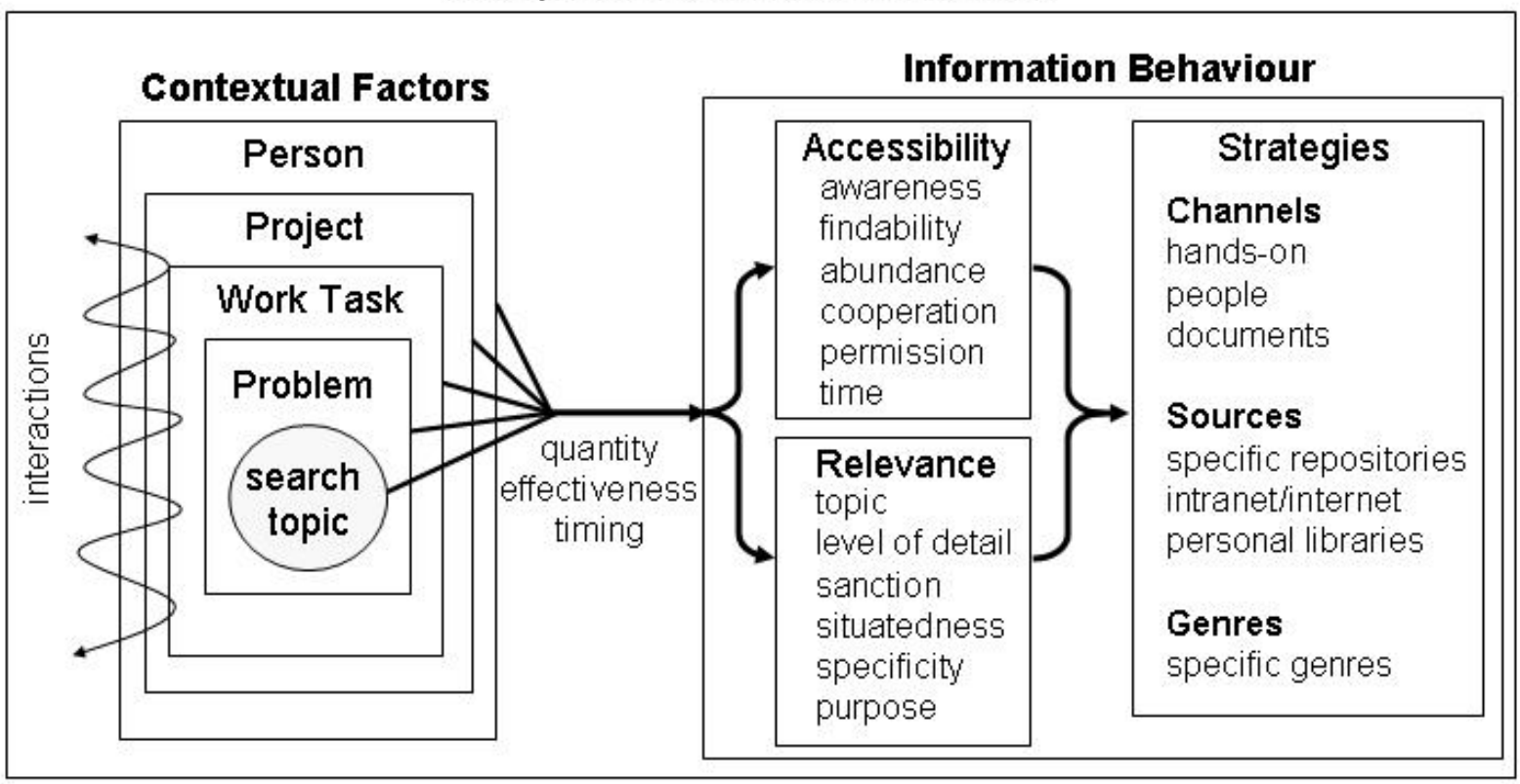

\section{Impact of context on information seeking in general}

In general, variation in contextual factors can be seen to influence the quantity, effectiveness and timing of information seeking activities. For example, expertise and familiarity are associated with more effective searching, certain tasks such as troubleshooting are associated with a greater 
amount of information seeking, and the role of the consultant is associated with the timing of information seeking in the framework of a project.

\section{Impact of context on information accessibility}

Contextual factors can both increase and limit access to information via constraints related to searchers, information objects, and the searching environment itself. Six dimensions of accessibility emerged from the interview data:

- Consultants' awareness of information;

- Findability: how easy information is to find;

- Abundance: the quantity of information available;

- Level of cooperation between experts sharing information;

- Consultants’ permissions with respect to access-controlled information;

- $\quad$ Time available to seek out and use information.

\section{Impact of context on relevance and utility criteria}

Contextual factors shape the goals of the searcher with respect to various information characteristics. These characteristics serve as the criteria for utility or situational relevance. In this domain, the highest utility value is ascribed to information that can be applied directly to the problem or task at hand, or even recycled “as-is” to serve as a project input or deliverable. Utility increases in importance under tight schedules, because the searcher has no time to adapt topically relevant information to suit the task at hand.

The set of information characteristics that emerged as context-dependent utility criteria are described below. The labels were assigned by the researcher, as interview participants expressed these concepts using a range of terms. 
- Subject matter - the topical content of the information. Consultants distinguish broadly between information about technology in a generic sense, such as Internet protocols or content management systems, and information about proprietary software products.

Usually, they are seeking information that is about the technologies and products with which they are working.

- Level of detail — the conceptual level, depth and scope of the information. "High level" and "low level" were frequently used to distinguish between information that is more abstract with a broader scope versus more concrete, specific and detailed information.

- Sanction-This characteristic refers to a distinction made by consultants between official, more formally "sanctioned" information objects such as product documentation versus unofficial and less formal sources such as discussion group postings or draft reports. Sanction is not simply an issue of authority, because in some cases, consultants consider unsanctioned sources to be more accurate and reliable.

- Situatedness - the degree to which an information object is an expression of situated knowledge, gained through the first-hand experience of the author, as opposed to a theoretical understanding of the material.

- Specificity - the degree to which information relates to a particular context or situation as opposed to generic information that is applicable to a broader set of circumstances.

- Purpose - a distinction between information created for different purposes, such as training, documenting, marketing or guiding.

These features make up the main selection criteria that consultants use to focus their search for information objects suited to particular situations. This is not a comprehensive set of relevance 
criteria. For example, authority, quality and currency are important information characteristics in almost all situations, and thus did not emerge from this analysis as context-dependent criteria.

\section{Impact of context on information search strategies}

By setting access constraints and establishing relevance criteria, contextual factors indirectly influence the strategies used by consultants to seek out information. Participants described a number of strategies based on three features of the information environment: channels, sources, and genres:

- Channels: choosing to seek information through hands-on experience, through people, or through documents. Consultants tend to try things out themselves when they have time and opportunities to learn new technologies. They turn to colleagues when they have very specific and unusual problems. One consultant noted that he turns to colleagues "when there are things that you cannot read from a book... the things that are based on a lot of experience” (P04). They also turn to colleagues to save time: "I would try to ask the consultant for sure. To answer this question might be a lot of reading, a lot of reading...” (P04). Documents were used to some degree in most other situations, and were often used to support person-to-person information exchanges and hands-on learning as well.

- Sources: choosing to use specific information sources, such as particular websites or repositories. Consultants have a clear sense of the different types of information stored in key repositories used by their group, and use this knowledge to find information suited to their needs. For example, one consultant referred to the intellectual capital repository as follows: "the only thing that's in there is customization .....of particular, very special scenarios that are not handled by base product documents". (P01) 
- Genres: seeking out particular document genres, such as best practices or technical articles. Looking for familiar genres is one of the most pervasive techniques used by consultants to find documents with particular qualities. For example, a consultant looking for procedural information noted, “[manuals] is also a good place too because they tend ... to just lay things out step by step” (P06). Furthermore, specific genres are often associated with particular tasks: "installation...definitely the manual, the information centre....development process - this is one area that we'll read the best practices.” (P04).

\section{Interaction effects between contextual factors}

Different contextual factors are clearly inter-related, and in many cases, the impact of context may be mediated through its impact on another factor. For example, the role of a consultant tends to constrain the work tasks they are likely to undertake, and familiarity with a subject area tends to increase as the stage of completion of a project increases.

\subsection{Summary and Next Steps}

The objective of this phase of the research was to identify and model the role of context in shaping information behaviour in this domain. The pre-study was used to collect data on work and information practices that informed the design of the contextual interviews conducted with members of the study population. By focusing the interview questions on a number of broad layers of context within this workplace domain, participants were encouraged to spontaneously refer to relationships between particular aspects of that context and their information practices. These references, which were scattered throughout the interviews, were identified, analyzed, grouped and coded, and used to determine the key contextual factors at play, the criteria and constraints guiding information selection, and the strategies employed to find necessary information. 
The data revealed a complex set of relationships between contextual factors and information searching and selecting behaviour. Within this complexity, however, specific, recurring topics and themes emerged.

The model suggests that when a consultant searches for information, a range of contextual factors determine the characteristics of the information sought and set some parameters that determine how accessible the information will be. The searcher then makes use of information strategies designed to find the right kind of information within the existing constraints. The main strategies are to choose between hands-on experience, people or documents, to make use of specific information repositories, or to seek out certain known genres of information. The model identifies six information characteristics that serve as context-dependent relevance criteria as well as six context-dependent accessibility parameters.

The findings presented above provide answers to the first two questions guiding this research. The key contextual factors are identified and the nature of their relationship with information behaviour in this domain is characterized, both at the level of individual factors and at the general level. However, the findings represent a highly complex environment involving a large number of factors and relationships, which would be very difficult to implement in a system framework. Furthermore, it would be premature to apply the model to system design, as it requires validation and testing. Therefore, in order to apply these findings, it was necessary to isolate a smaller number of inter-related factors and focus on validating and testing a more restricted contextual model.

One of the strongest and best-documented relationships that emerged from the interview data is between the tasks consultants were trying to accomplish and the document genres they reported seeking out and using. Although the relationship between tasks and genres is not direct, since genre seeking was generally used as a strategy to find information with certain specific 
characteristics, the associations between particular tasks and genres seem to be very strong. Furthermore, task and genre types are pronounced features of this work and information environment. Consultants tend to describe their work in terms of their work tasks, and genre is frequently used as a means of organizing documents, databases and repositories. Based on the strong association between task and genre variables as well as the prevalence of task and genre categories in this domain, they were identified as candidates for an implementation of contextual retrieval. The next phases of this research focus on this relationship between task and genre. Validating and testing the other relationships suggested by the model will be set aside for future work. 


\section{Task-Genre Relationships}

\subsection{Overview}

Chapter four presented a model of the contextual effects on searching and selecting behaviour in a software engineering work domain. The model maps out a landscape in which specific hypotheses can be identified, explored and tested. With respect to document selection, it also points to a strong relationship between two variables typically considered to be external to the core IR processes: Task and Genre. In this chapter, the scope of inquiry narrows to focus on these two variables, with the aims of validating, characterizing, and operationalizing the relationship between tasks and genres in this domain. Three specific research questions guided this phase of the study:

RQ3: What are the main categories and characteristics of tasks and of genres in this domain?

RQ4: Does a significant relationship exist between task and genre in this domain?

RQ5: Are there patterns of association among the categories of these variables that can be identified and exploited for IR?

A series of studies were designed to respond to these research questions. The first explored the extent and nature of the relationship between tasks and genres as expressed through metadata in a large document repository in use within this domain. The second resulted in the identification of the main task and genre categories in the form of domain-specific task and genre taxonomies. 
The third study validated the characteristics and relationships of the variables further through an online survey of the study population. The chapter begins with a discussion of the nature of the task - genre relationship as suggested by the model presented in the previous chapter. The three studies are presented in separate sections to follow.

\subsection{Task and Genre}

The model developed in Chapter Four identified a strong, but indirect relationship between task and genre. The concept of task breaks down into two types of tasks: work tasks and information tasks. In general, tasks are activities undertaken to achieve goals. In this setting, work task is defined at a high level to reflect the target population's practice of distinguishing between broad categories of professional activities such as migration or troubleshooting. Work tasks defined at this level may vary considerably with respect to task characteristics such as complexity, difficulty, predictability, etc., and each work task type may include a large number of sub-tasks. Information tasks are typically prompted by work tasks, and are categorized according to the cognitive goals associated with seeking information, such as making a decision or learning. When a problem arises and prompts information seeking activity, the work task and the embedded information task intersect to form a two-dimensional task classification of the search. For example: in conducting a system migration, a consultant may need to learn more about a competing product.

Within this model, genre is represented as a perceived meta-characteristic of documents, which searchers use strategically to identify documents likely to possess certain combinations of more specific characteristics. For example, in the case of a consultant conducting a product installation, who needs detailed step-by-step, sanctioned and authoritative instructions, he is likely to try to find information in the form of product documentation, which is known to have these characteristics. Alternately, if a consultant wanted a colleague’s opinion, based on situated 
knowledge, he might prefer to retrieve discussion threads from an internal company news group. In other words, consultants use genres, which are informal and constructed categories of information objects within their community, as implicit short cuts to finding information objects with the characteristics (level of specificity, purpose, etc.) suited to particular situations. Tasks are related to genres because they determine which information characteristics are needed.

\subsection{Exploration of Task and Genre Relationships}

In response to Research Questions Four and Five, concerning the strength and nature of the taskgenre relationship, I designed a study to test the hypothesis that Task and Genre are not independent. A dataset of metadata was extracted from one of the main information repositories used by the study population. The IC Repository (ICR) contains documents recommended and/or authored and manually indexed with task and genre metadata tags by the consultants themselves. Analysis of the repository was based on the assumption that patterns of co-occurrence between these task and genre metatags is indicative of a more general relationship between these variables with respect to how information is selected and used in this environment.

\subsubsection{Method}

\section{i. Dataset}

The ICR is a restricted access Lotus Notes database that contained 3400 documents at the time of the study, ranging from brief notes to lengthy review articles. While a broad range of materials are included, it represents a subset of information resources used in the overall work domain. The process of submitting items to this repository includes the completion of a metadata form with thirty data fields. Alongside the more common factual tags such as author, date submitted, and title, are two interpretive tags, artifact type and purpose. Artifact type identifies the document type or genre of an item, for example: whitepaper, presentation, collection, etc. In most cases, consultants assigned a single artifact type to each document. Purpose identifies the intended use of an item, which is representative of the task/s that a document is capable of supporting. More 
than one purpose tag were frequently assigned to each document. These metadata tags served as surrogates to test the task-genre relationship.

The initial dataset consisted of 6400 pairs of Task (purpose) and Genre (artifact type) tags relating to items in the repository. However, despite apparent attempts to control data entry to sets of about 16 controlled values for each field, there were actually 47 different Genre categories and 125 different Task categories in evidence. Prior to conducting the analysis, this was reduced to a more concise set of 17 Genres and 36 Tasks (Table 5-1) by grouping sub-categories together under more general headings, and removing low frequency categories.

Table 5-1: Task and Genre ICR Metadata Categories and Frequencies

\begin{tabular}{|c|c|c|c|c|c|}
\hline Genres (17) & Freq & Work Tasks (20) & Freq & $\begin{array}{c}\text { Information } \\
\text { Tasks (16) } \\
\end{array}$ & Freq \\
\hline reading material & 1629 & administrate/install & 332 & educate & 1903 \\
\hline presentation & 1100 & architecture/design & 263 & demonstrate & 484 \\
\hline cookbook & 713 & integration & 216 & document & 420 \\
\hline architecture/design & 545 & migration & 172 & example & 139 \\
\hline source code & 497 & performance tuning & 164 & technical info & 115 \\
\hline demo & 339 & debugging & 153 & market & 93 \\
\hline lecture/lab & 228 & development & 145 & tool & 80 \\
\hline collection & 218 & installation & 134 & support & 71 \\
\hline engagement summary & 126 & implementation & 125 & reference & 70 \\
\hline discussion & 101 & configuration & 96 & compare & 45 \\
\hline product feedback & 95 & competitive evaluation & 86 & method & 38 \\
\hline website/repository & 82 & security & 62 & standards & 13 \\
\hline sales kit & 52 & deployment & 61 & index & 12 \\
\hline schedule & 20 & test & 57 & guide & 9 \\
\hline tools & 19 & project management & 51 & roadmap & 5 \\
\hline legal material & 19 & proof of concept & 49 & contacts & 4 \\
\hline white paper & 9 & project review & 43 & & \\
\hline & & discovery session & 41 & & \\
\hline & & product presentation & 21 & & \\
\hline & & capacity planning & 20 & & \\
\hline
\end{tabular}

For example, presentation/lecture was included under presentation and the one install tip was removed. The final dataset contained about 5800 pairs of tags. In order to avoid introducing bias, category labels assigned by the consultants were retained whenever possible, even though they are ambiguous in some cases. For example, the tool/s and architecture/design categories appear both as Genres and Tasks. The Task field seems to have been interpreted in two different ways by repository users: about half the categories represent work tasks, while the other half represent 
a variety of information tasks. For purposes of interpretation, these were divided these into two conceptual groups (see Table 5-1), but the analysis treated all 36 Tasks as categories of a single variable.

\section{ii. Data Analysis}

Data was analyzed using SPSS. A cross-tabulation analysis was conducted to test for a relationship between the two variables. The analysis used a contingency table of observed and expected frequencies for each of the 36x17 task-genre combinations to calculate the Chi Square statistic and measures of association. Because the number of items in each Genre category varied widely (see frequencies in Table 5-1) the contingency table is quite sparse, with a high percent of empty cells. As this violates one of the assumptions of the Chi Square test, Monte Carlo tests were conducted in order to produce unbiased estimates of the significance level. The standardized residuals of observed and expected frequencies were analyzed to identify relationships between specific categories of tasks and genres.

Due to the difficulty of interpreting patterns in such a large contingency table, a correspondence analysis was also conducted. Correspondence analysis (CA) tests the relationship between categorical variables by calculating the associations between and among row and column values in two-way contingency tables based on a measure of distance between points (categories). It uses a Chi-Square distribution to determine the independence of cells and the similarity/ dissimilarity of row and column variables, and represents them graphically in a low dimensional space (Greenacre, 1984). The goals of CA are similar to other multivariate analyses such as factor analysis and principle components analysis which are used for dimension reduction. However, where factor analysis determines which variables cluster together, CA identifies clusters of categories within variables. CA is robust for large sparse contingency tables, and is therefore well suited to this dataset (Greenacre, 2007). Symmetrical normalization was used to prepare the CA maps, since this method plots the tasks and genres together into the same space, and allows 
for visual interpretation of the relationships between them. Using this approach means that although proximity between row and column variables indicates association, the distances between them cannot be interpreted as measured strengths of association.

\subsubsection{Results}

\section{i. Cross Tabulation Analysis}

Results of the cross tabulation analysis support the hypothesis that Task and Genre are not independent. Both the Pearson's Chi Square statistic $\left(x^{2}=5878.968, d f=560, p<.001\right)$ and the Monte Carlo Test $(\mathrm{p}<.001)$ are significant. Several measures of association for nominal data are calculated by SPSS. The Cramer's V value of .252 (range 0 to +1 ) indicates that while the relationship is significant, it is not particularly strong. Lambda (.053) and Goodman and Kruskal tau (.072) values indicate that if Genre is the dependent variable, knowing the Task would result in a proportional reduction of error in assigning categories in the range of $5.3 \%$ to $7.2 \%$.

Nevertheless, the cross tabulation output (Appendix 3) showing the observed vs. expected frequencies for each combination of task and genre categories, indicates a substantial amount of variation. Table 5-2 provides a summary of Genres that co-occur more or less frequently than expected with each Task. All Genres with standardized residuals of more than twice the standard deviation (+/-2) are included in the table, and those more than five times SD (+/-5) are bolded. 
Table 5-2: Summary of higher and lower than expected co-occurrences of task and genre categories*

\begin{tabular}{|c|c|c|}
\hline Task/Purpose & Frequently Co-occurring Genres & Infrequently Co-occurring Genres \\
\hline \multicolumn{3}{|l|}{ Work Tasks } \\
\hline administrate/install & $\begin{array}{l}\text { cookbook, product feedback, source } \\
\text { code, website/repository }\end{array}$ & $\begin{array}{l}\text { discussion, lecture/lab, presentation, } \\
\text { reading material }\end{array}$ \\
\hline architecture & architecture/design, presentation, & $\begin{array}{l}\text { cookbook, engagement summary, } \\
\text { lecture/lab, source code }\end{array}$ \\
\hline capacity planning & discussion, website/repository & \\
\hline $\begin{array}{l}\text { competitive } \\
\text { evaluation }\end{array}$ & discussion, engagement summary & $\begin{array}{l}\text { architecture/design, cookbook, source } \\
\text { code }\end{array}$ \\
\hline configuration & cookbook, source code, white paper & architecture/design,. presentation \\
\hline debugging & $\begin{array}{l}\text { demo, product feedback, source code,, } \\
\text { website/repository }\end{array}$ & lecture/lab, presentation \\
\hline deployment & source code, white paper & presentation \\
\hline development & source code & \\
\hline discovery session & $\begin{array}{l}\text { engagement summary, legal material, } \\
\text { tools }\end{array}$ & \\
\hline implementation & reading material & presentation \\
\hline installation & cookbook, demo & $\begin{array}{l}\text { architecture/design, lecture/lab, } \\
\text { presentation }\end{array}$ \\
\hline integration & $\begin{array}{l}\text { architecture/design, cookbook, source } \\
\text { code, tools }\end{array}$ & lecture/lab, presentation \\
\hline migration & engagement summary & lecture/lab \\
\hline performance tuning & demo, discussion & lecture/lab \\
\hline proof of concept & engagement summary & presentation \\
\hline product presentation & presentation & \\
\hline project management & $\begin{array}{l}\text { engagement summary, sales kit, } \\
\text { schedule }\end{array}$ & $\begin{array}{l}\text { presentation, reading material, source } \\
\text { code }\end{array}$ \\
\hline product review & collection & presentation \\
\hline security & architecture/design, cookbook & \\
\hline test & demo & architecture/design \\
\hline \multicolumn{3}{|l|}{ information tasks } \\
\hline compare & architecture/design, schedule & \\
\hline contacts & collection, website & \\
\hline demonstrate & cookbook, demo, source code & $\begin{array}{l}\text { presentation, product feedback, reading } \\
\text { material }\end{array}$ \\
\hline document & $\begin{array}{l}\text { architecture/design, collection, } \\
\text { discussion, product feedback, reading } \\
\text { material }\end{array}$ & $\begin{array}{l}\text { demo, lecture/lab, presentation, source } \\
\text { code }\end{array}$ \\
\hline educate & $\begin{array}{l}\text { lecture/lab, presentation, reading } \\
\text { material }\end{array}$ & $\begin{array}{l}\text { architecture/design, cookbook, demo, } \\
\text { discussion, engagement summary, } \\
\text { product feedback, sales kit, source code }\end{array}$ \\
\hline example & lecture/lab, source code & presentation \\
\hline guide & reading material & \\
\hline index & collection, website & \\
\hline market & $\begin{array}{l}\text { engagement summary, legal material, } \\
\text { sales kit }\end{array}$ & $\begin{array}{l}\text { architecture/design, reading material, } \\
\text { source code }\end{array}$ \\
\hline method & reading material & \\
\hline reference & $\begin{array}{l}\text { collection, legal material, schedule, } \\
\text { website }\end{array}$ & demo, presentation \\
\hline standards & architecture/design & \\
\hline support & reading material & presentation, source code \\
\hline tech info & $\begin{array}{l}\text { presentation, reading material, white } \\
\text { paper }\end{array}$ & $\begin{array}{l}\text { architecture/design, cookbook, demo, } \\
\text { lecture/lab, source code }\end{array}$ \\
\hline
\end{tabular}




\begin{tabular}{l|l|l}
\hline tool & source code, white paper & $\begin{array}{l}\text { architecture/design, cookbook, } \\
\text { presentation }\end{array}$ \\
\hline
\end{tabular}

* All Genres with standardized residuals of more than twice the standard deviation (+/-2) are included in the table. Those with more than five times SD (+/-5) are bolded.

\section{ii. Correspondence Analysis}

CA offers a means of exploring two aspects of the Task-Genre relationship that are not covered in the cross tabulation analysis: the underlying factors influencing task-genre variation, and relationships between categories within the variables. The CA output summary is presented in Table 5-3 below.

Table 5-3: SPSS Summary of ICR Correspondence Analysis

\begin{tabular}{|c|c|c|c|c|c|c|c|c|c|c|}
\hline \multirow{3}{*}{ Dim } & \multirow{3}{*}{$\begin{array}{l}\text { Singular } \\
\text { Value } \\
\end{array}$} & \multirow{3}{*}{ Inertia } & \multirow{3}{*}{$\begin{array}{l}\text { Chi } \\
\text { Square }\end{array}$} & \multirow{3}{*}{ Sig. } & & & \multicolumn{4}{|c|}{ Confidence Singular Value } \\
\hline & & & & & \multicolumn{2}{|c|}{ Proportion of Inertia } & \multirow[b]{2}{*}{$\begin{array}{l}\text { Standard } \\
\text { Deviation } \\
\end{array}$} & \multicolumn{3}{|c|}{ Correlation } \\
\hline & & & & & $\begin{array}{l}\text { Accounted } \\
\text { for }\end{array}$ & Cumulative & & 2 & 3 & 4 \\
\hline 1 & 0.488 & 0.238 & & & 0.234 & 0.234 & 0.030 & 0.659 & 0.389 & 0.006 \\
\hline 2 & 0.451 & 0.203 & & & 0.200 & 0.434 & 0.015 & & 0.361 & 0.017 \\
\hline 3 & 0.380 & 0.144 & & & 0.142 & 0.576 & 0.024 & & & 0.114 \\
\hline 4 & 0.276 & 0.076 & & & 0.075 & 0.651 & 0.019 & & & \\
\hline 5 & 0.266 & 0.071 & & & 0.070 & 0.721 & & & & \\
\hline 6 & 0.251 & 0.063 & & & 0.062 & 0.783 & & & & \\
\hline 7 & 0.240 & 0.058 & & & 0.057 & 0.840 & & & & \\
\hline 8 & 0.206 & 0.043 & & & 0.042 & 0.882 & & & & \\
\hline 9 & 0.173 & 0.030 & & & 0.030 & 0.912 & & & & \\
\hline 10 & 0.168 & 0.028 & & & 0.028 & 0.940 & & & & \\
\hline 11 & 0.154 & 0.024 & & & 0.023 & 0.963 & & & & \\
\hline 12 & 0.134 & 0.018 & & & 0.018 & 0.981 & & & & \\
\hline 13 & 0.089 & 0.008 & & & 0.008 & 0.989 & & & & \\
\hline 14 & 0.072 & 0.005 & & & 0.005 & 0.994 & & & & \\
\hline 15 & 0.057 & 0.003 & & & 0.003 & 0.997 & & & & \\
\hline 16 & 0.055 & 0.003 & & & 0.003 & 1.000 & & & & \\
\hline Total & & 1.015 & 5878.968 & 0.000 & 1.000 & 1.000 & & & & \\
\hline
\end{tabular}

a. 560 degrees of freedom

The summary indicates that the full model with the maximum possible number of dimensions

(16) accounts for $100 \%$ of the total inertia (variation from random association), indicating that it

is a strong relationship overall. The first six dimensions account for about $77 \%$ of the inertia.

Typically one to three dimensions account for this much variance in a correspondence analysis 
with fewer categories, but this result is comparable to similar analyses of large matrices (AskellWilliams \& Lawson, 2004).

CA uses correspondence maps to graphically represent the variation between and among the categories of the variables, broken down into a number of dimensions. The challenge in doing this analysis is to interpret the maps and assign class concepts to the dimensions, which represent the underlying source of the variation. The second column in Table 5-3 reports the correlation (analogous to the Pearson correlation coefficient) between the row and column scores for each dimension (Singular Value Score). This analysis will consider the first three dimensions, which have inertia of greater than 1. Plotting the singular values in a Scree plot (Figure 5-1) confirms that after the third dimension, values begin to drop less rapidly and are similar in size, indicating that the first three dimensions of variation should be considered.

Figure 5-1: Scree plot of singular values

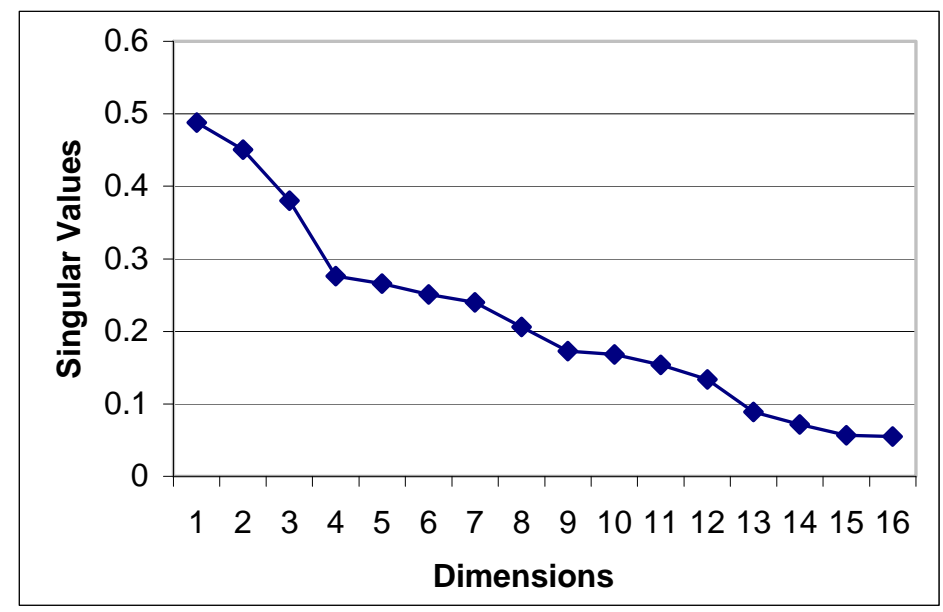

The graphical output of the 3-dimensional solution consists of two correspondence maps, which are biplots (Figures 5-2 to 5-3) showing the first and strongest dimension (along the x-axis) by each of the remaining two dimensions (along the y-axis). Some labels are hidden for legibility. In interpreting these graphs, it is important to recognize that not all points (categories) contribute equally to the inertia or variation of each dimension; therefore, determining the relative 
contributions of different points supports more accurate interpretation. This can be done by calculating the average contribution of row and column points per dimension (1/number of points) and comparing this with the contribution to inertia for each point in each dimension.

Points that make a higher than average contribution, play a dominant role in determining variation in that dimension (Askell-Williams \& Lawson, 2004).

Figure 5-2: Correspondence Map of Dimensions 1 and 2

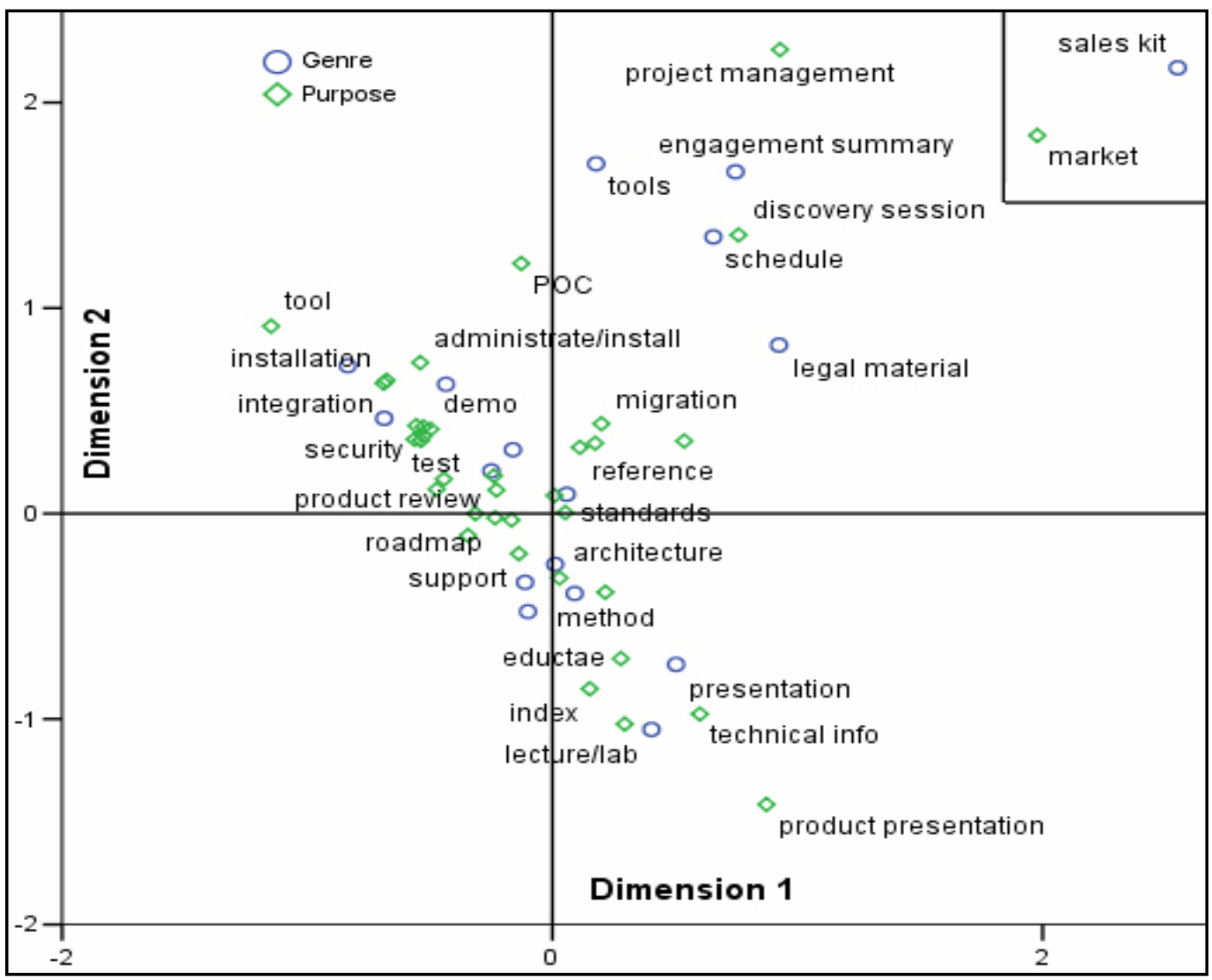

*datapoints in upper right were shifted to appear on the graph

\section{Dimension 1: Variability based on Work Activities}

The first dimension of variability is represented by the horizontal distribution of points along the $\mathrm{x}$-axis in Figures 5-2 and 5-3. A plausible interpretation of the underlying cause of this variation is the distinction between the different types of work tasks in which software consultants are 
involved. The left quadrants in all three figures contain points related to software engineering: development, deployment, etc. Points in the right quadrants are related to consulting work: project management, schedule, etc. Finally, there is another cluster of two points—marketing and sales kits—far in the upper right quadrant that represent yet another work activity: sales. These can be considered outliers in this data, as they are clearly related to one another, but do not have a strong connection with the rest of the materials in this repository. This is not surprising, as the consultants in this group do very little sales work. Looking at the data points with above average contributions to inertia supports this interpretation. Some strong genre points (> .058 contribution to inertia) are source code (.123) and sales kit (.613), and some strong task points (> .028) are administrate (.034), integration (.035) and demonstrate (.042).

\section{Dimension 2: Variability based on Level of Detail}

The second dimension of variability in associations between genres and tasks in this domain is represented by the vertical distribution of points along the y-axis in Figure 5-2. This dimension can be interpreted as the distinction between low level technical and high level conceptual orientations. More technical and procedural tasks and information types related to both software engineering and consulting appear in the upper quadrants. Some of the stronger points are cookbooks (.108), source code (.099) and engagement summaries (.134). In the lower quadrants, data points represent more generic sources of information and learning as well as some higher level, conceptual tasks, such as architecture and capacity planning. The strongest points in the lower quadrants are all related to teaching and learning: educate (.363), presentation (.227) and lecture/lab (.096). There are no strong points associated with the lower left quadrant which represents high level /software engineering. The few data points that appear, however, do support the interpretation: guide, website and white paper. 


\section{Dimension 3: Variability based on Work Roles}

The third dimension of variability is represented by the vertical distribution of points along the yaxis in Figure 5-3.

Figure 5-3: Correspondence Map of Dimensions 1 and 3

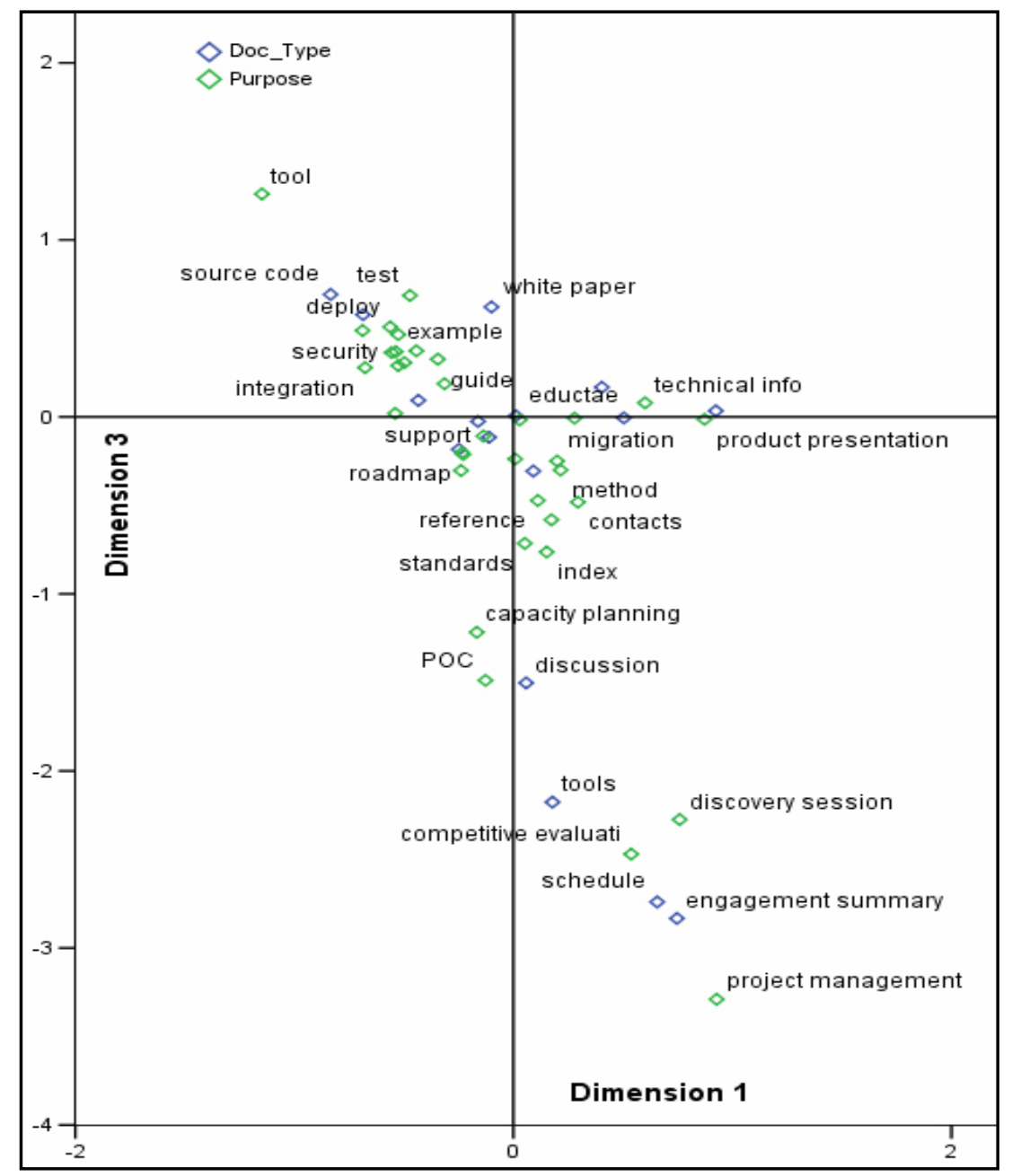

There is a strong correlation between the first and third dimensions, which can be seen by the shape of the data. Most of the points are in the upper left (software engineering) and lower right (consulting) quadrants. However, the strongest points in the dimension point to a sharper distinction between various project roles which consultants in this group typically perform: 
project manager, architect/designer and technical specialist. The strong points project management (.251) and engagement summary (.460) are closely associated with the first role, and related points form a clear cluster in the lower right. There is another loose cluster just below the centroid, which seems to relate to the second role, as it includes a number of high level design and planning tasks and related information types: architecture, design docs, capacity planning, standards, and roadmap. Finally, on the far upper left is another cluster that includes most of the software engineering tasks, but the strongest points relate to types of technical tasks and information that would be used by technical specialists: source code (.108), tool (.058) and demo $(.052)$.

\section{Genre Clusters}

In addition to the broad patterns of association identified through analysis of the correspondence maps, there are some smaller groupings of points that tend to be strongly associated throughout the various dimensions. By plotting the row points (Genres) separately (Figure 5-4), a number of these small groupings become more apparent. Interpretation of these clusters suggests some micro patterns of association within the Genre categories, described below:

a. Project Reusables (tools, engagement summary, schedule, legal material). This cluster relates to consulting work. They are all examples of genres that would be used primarily as templates and reusable information objects for successive consulting projects. These materials are closely associated with the task project management.

b. Low-level technical (source code, cookbook, demo). These materials all contain low-level, concrete and specific technical information. This type of information is closely associated with a grouping of procedural tasks with a similar low-level profile: installation, configuration, deployment, and administrate. 
Figure 5-4: Genre Clusters

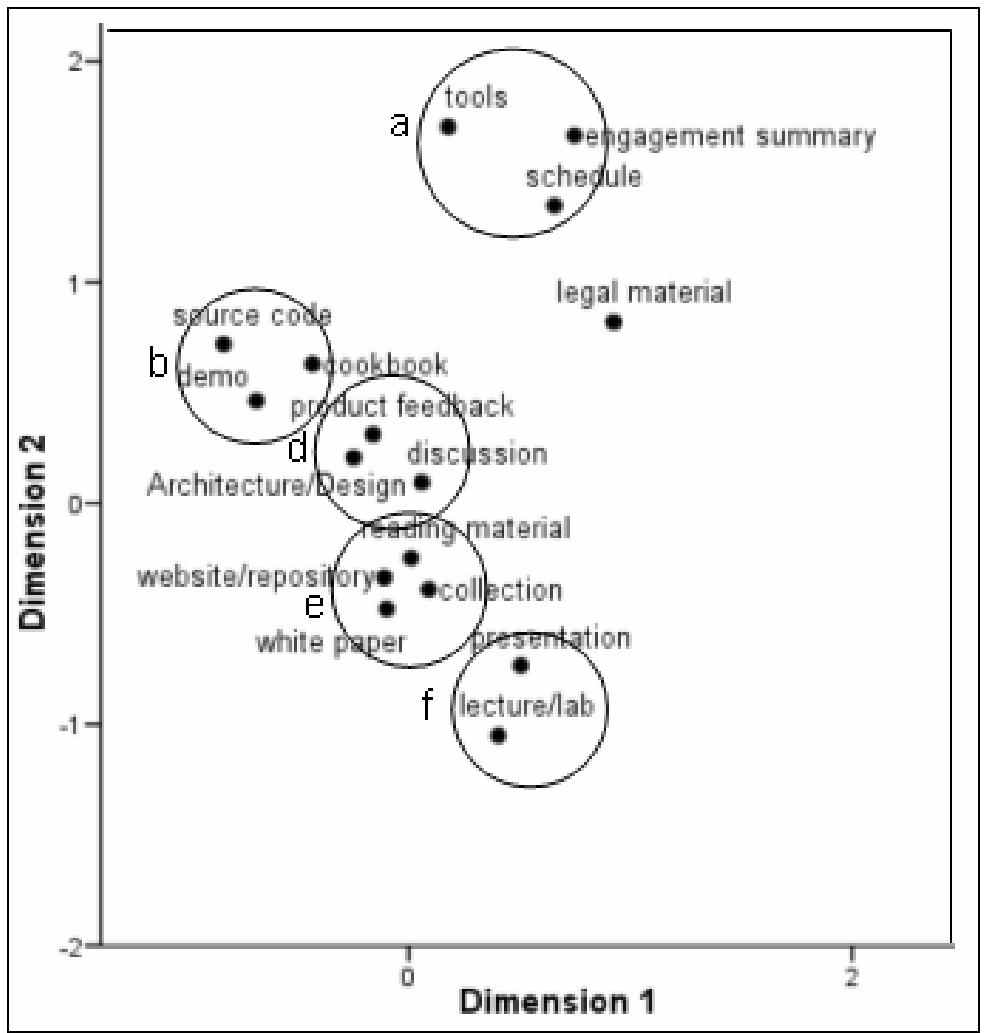

c. System data (source code, demo, tool ). This cluster (not shown in Figure 5-4) relates to system-generated information rather than secondary documentation and seems to be related to debugging and performance tuning. In some dimensions, this cluster appears together with more development-oriented software engineering tasks, but in others it appears as a distinct cluster.

d. Product maintenance (product feedback, design documents, discussion threads). This cluster has a product focus, and tends to straddle the axis between software engineering and consulting. Related tasks are migration and support.

e. High-level generic (reading material, website, white paper, collection). This cluster includes materials that consultants would use to find conceptual rather than procedural information about technologies and products. Associated tasks are high-level and few in number: architecture and capacity planning. 
f. Educational (presentation, lecture/lab). These materials are closely related to one another and to the information task educate, but they do not have a strong associations with any particular work task or with either software engineering or consulting.

\subsubsection{Summary and Discussion of Exploration Study}

Results support the hypothesis that Task and Genre are not independent by showing a significant relationship between these variables in the form of metadata tags assigned to items in this repository. Although measures of association indicate that the relationship is not very strong, the actual and expected frequencies show considerable variation, and clearly identify small sets of genres that are related to all but one of the Work Tasks and Information Tasks in the collection. For more than half the tasks, one or more Genres co-occur very frequently (more than 5 times SD), suggesting the existence of strong specific relationships. It is interesting to note that the positive associations seem to be more robust and varied than the negative associations, which are fewer and are dominated by a number of Genre categories with very high frequencies in the dataset (presentation, lecture/lab and source code). The lack of a strong association overall may reflect the fact that for many of the Tasks only one or two Genres are related, and some Genres are only related to one or two Tasks.

Interpretation of the correspondence maps resulted in the identification of factors underlying the task-genre association. The strongest factor that emerged is the type of work activity, of which three types were in evidence in this dataset: software engineering, consulting and marketing. Certain task sets and genre repertoires are associated with each of these work activities and as a result, those tasks and genres cluster together. The next strongest factor is related to the level of detail of tasks and genres. Tasks performed at a certain level of detail seem to require information sources at the same level, and level of detail serves as an important distinction between tasks and genres in this domain. The third factor identified is an extension of the first; a finer-grained distinction between the roles performed within the software engineering set of work 
activities. Findings are well-aligned with the workplace domain study, which also pointed to the importance of roles, tasks, and the level of detail. Understanding the underlying factors of this relationship is important, as it suggests a means of generalizing the task-genre relationship for application in other professional environments.

\subsection{Taxonomy Development}

The dataset for the previous study was drawn from a single repository of shared intellectual capital, in which the Task and Genre categories were established by the user community. While the data provides evidence of the types of tasks and genres in use, the sets of categories defined in the repository does not represent the full range of activities of the study population. In order to further study and exploit the task-genre relationship in this domain in a more controlled manner, I developed simple taxonomies in the form of sets of named and defined categories of the most prevalent work tasks, information tasks and genres. A bottom-up, user-centred approach to taxonomy development was taken to ensure that the taxonomies would reflect actual work practices and information behaviour.

\subsubsection{Task Taxonomies}

Lists of common work and information tasks were first compiled during the workplace domain pre-study based on the tasks mentioned by participants (see Table 4-1). These lists were refined based on feedback from interview participants, who were shown the draft task taxonomies and asked to comment. The guiding principles for the design of the final task taxonomies were pragmatic: they needed to illustrate the study population's understanding of their work practices and provide an easy means of identifying the tasks associated with a given search situation. The goal was to produce sets of common tasks labelled in terms both meaningful and unambiguous to the target population. In order to reduce complexity, hierarchical relationships between tasks and sub-tasks and distinctions between software engineering and consulting tasks were not expressed 
in the final taxonomies. The final work task taxonomy includes 11 categories, and the final information task taxonomy includes five categories. The categories and definitions for each are presented in Table 5-4.

Table 5-4: Work Task and Information Task Taxonomies

\begin{tabular}{|c|c|}
\hline Work Task Types & Definition \\
\hline Architecture & $\begin{array}{l}\text { determining the components of a computer system and the way } \\
\text { they interact with one another }\end{array}$ \\
\hline Deployment & $\begin{array}{l}\text { the process of placing an application in a distributed environment } \\
\text { and making the application available for use }\end{array}$ \\
\hline Design & $\begin{array}{l}\text { researching, designing, and specifying the logical function of an } \\
\text { application, environment or process. }\end{array}$ \\
\hline Implementation & building the target program \\
\hline Installation \& Configuration & $\begin{array}{l}\text { getting software running on a system and adjusting the software } \\
\text { settings }\end{array}$ \\
\hline Integration & $\begin{array}{l}\text { making separate software/hardware systems and devices function } \\
\text { together }\end{array}$ \\
\hline Migration & moving a system from one product or technology to another \\
\hline Performance Tuning & $\begin{array}{l}\text { testing and adjusting the system to increase its processing speed, } \\
\text { load and reliability }\end{array}$ \\
\hline Project Management & $\begin{array}{l}\text { planning and coordinating a project with the aim of meeting } \\
\text { requirements and ensuring timely completion, within cost and } \\
\text { quality standards. }\end{array}$ \\
\hline Proof of Concept & $\begin{array}{l}\text { design, implement and demonstrate a working software solution to } \\
\text { a business process or problem }\end{array}$ \\
\hline Troubleshooting & $\begin{array}{l}\text { using strategies to define and solve problems encountered during } \\
\text { the use of computer systems }\end{array}$ \\
\hline Information Task Types & Definition \\
\hline Learn about a topic & $\begin{array}{l}\text { trying to learn about an unfamiliar topic: seeking general } \\
\text { orientation and an understanding of concepts. }\end{array}$ \\
\hline Make a decision & $\begin{array}{l}\text { trying to make a decision: identification and comparison of } \\
\text { alternatives in order to determine a course of action }\end{array}$ \\
\hline Find out how to & $\begin{array}{l}\text { trying to find out how to do something - a procedure or work plan } \\
\text { identifying the steps to take and issues involved. }\end{array}$ \\
\hline Find facts & $\begin{array}{l}\text { trying to find specific factual information about products or } \\
\text { technologies, for example: parameter values or supported software. }\end{array}$ \\
\hline Find a solution & $\begin{array}{l}\text { trying to solve a problem or fix a malfunction; finding information } \\
\text { on similar scenarios, problems, bugs and solutions. }\end{array}$ \\
\hline
\end{tabular}

\subsubsection{Genre Taxonomy}

The goals in developing a genre taxonomy were similar: to identify a core set of genres, small enough to be manageably implemented in a retrieval system, but with a scope broad enough to include a large portion of the documents in heavy use by this group. In order to preserve the 
domain-specific, situated nature of genre use and of existing task-genre relationships, a bottom-up classification approach was used, based on a study of the current information environment and commonly used genres. A list of all the genres mentioned by participants in the focus group and interviews conducted during the workplace domain study was compiled and combined with six genre taxonomies in use in websites and repositories in heavy use by the study population. This produced a list of 65 unique genres, more than half of which were found in only one repository or user-identified source. Some of the less common genres were specialized sub-genres, such as customer support plans, while others, such as sales kits, were less common in this particular domain. Table 5-5 lists the 28 genres identified in more than one source, showing the distribution across sources and the total frequency of occurrence for each.

The next step was to reduce the set to a smaller and more functional taxonomy, which could be used for classification of a workplace document collection. Selection was based primarily on genre prevalence, measured by the frequency of occurrence in Table 5-5. However, some additional practical considerations were taken into account:

- dominant sub-genres were preferred over very broad catch-all categories, which would be harder to characterize and classify (i.e. engagement summary was preferred over engagement materials, tutorial/lab was preferred over course and training materials, and product page was preferred over website);

- textual information genres were preferred over software tool genres, such as source code, tools, and reusable assets, as these types of files would be difficult to classify using textbased automatic classification.

The set was reduced to 16 genres, which are marked in bold in Table 5-5. 
Table 5-5: Distribution of Common Genres by Source*

\begin{tabular}{|c|c|c|c|c|c|c|c|c|c|}
\hline \multirow[t]{2}{*}{ Genre Class } & \multicolumn{6}{|c|}{ Genres used in Repositories (R 1-6) } & \multicolumn{2}{|c|}{$\begin{array}{l}\text { User-Identified } \\
\text { Genres }\end{array}$} & \multirow[b]{2}{*}{ Total } \\
\hline & $\mathrm{R} 1$ & R2 & R3 & R4 & R5 & R6 & Interviews & $\begin{array}{l}\text { Focus } \\
\text { Group }\end{array}$ & \\
\hline $\begin{array}{r}\text { courses, training } \\
\text { materials }\end{array}$ & $\mathrm{X}$ & & $\mathrm{X}$ & & & $\mathrm{X}$ & $\mathrm{X}$ & $\mathrm{X}$ & 5 \\
\hline manuals & $\mathrm{X}$ & & $\mathrm{X}$ & & $\mathrm{X}$ & & $\mathrm{X}$ & $\mathrm{X}$ & 5 \\
\hline presentations & $\mathrm{X}$ & & $\mathrm{X}$ & & & $\mathrm{X}$ & $\mathrm{X}$ & $\mathrm{X}$ & 5 \\
\hline product documents & $\mathrm{X}$ & & $\mathrm{X}$ & & $\mathrm{X}$ & & $\mathrm{X}$ & $\mathrm{X}$ & 5 \\
\hline technotes, tips & $\mathrm{X}$ & & & & $\mathrm{X}$ & $\mathrm{X}$ & $\mathrm{X}$ & $\mathrm{X}$ & 5 \\
\hline tutorials and labs & $\mathrm{X}$ & & $\mathrm{X}$ & & & $\mathrm{X}$ & $\mathrm{X}$ & $\mathrm{X}$ & 5 \\
\hline white papers & $\mathrm{X}$ & & $\mathrm{X}$ & & $\mathrm{X}$ & $\mathrm{X}$ & $\mathrm{X}$ & & 5 \\
\hline best practices & $\mathrm{X}$ & & $\mathrm{X}$ & & & & $\mathrm{X}$ & $\mathrm{X}$ & 4 \\
\hline design patterns & & $\mathrm{X}$ & $\mathrm{X}$ & $\mathrm{X}$ & & & $\mathrm{X}$ & & 4 \\
\hline discussions / forums & & & $\mathrm{X}$ & & $\mathrm{X}$ & & $\mathrm{X}$ & $\mathrm{X}$ & 4 \\
\hline engagement materials & $\mathrm{X}$ & & $\mathrm{X}$ & & & & $\mathrm{X}$ & $\mathrm{X}$ & 4 \\
\hline FAQs & $\mathrm{X}$ & & & & $\mathrm{X}$ & $\mathrm{X}$ & $\mathrm{X}$ & & 4 \\
\hline websites & $\mathrm{X}$ & & $\mathrm{X}$ & & & & $\mathrm{X}$ & $\mathrm{X}$ & 4 \\
\hline cookbooks \& guides & & $\mathrm{X}$ & $X$ & & & & $\mathrm{X}$ & & 3 \\
\hline demos & $\mathrm{X}$ & & $\mathrm{X}$ & & & & $\mathrm{X}$ & & 3 \\
\hline engagement summaries & & $\mathrm{X}$ & $\mathrm{X}$ & & & & $\mathrm{X}$ & & 3 \\
\hline problem reports & & & & & $\mathrm{X}$ & & $\mathrm{X}$ & $\mathrm{X}$ & 3 \\
\hline reusable assets & & $\mathrm{X}$ & $\mathrm{X}$ & & & & $\mathrm{X}$ & & 3 \\
\hline solutions & & & & $\mathrm{X}$ & $\mathrm{X}$ & & $\mathrm{X}$ & & 3 \\
\hline source code & & $X$ & $X$ & & & & & $X$ & 3 \\
\hline technical articles & $\mathrm{X}$ & & $\mathrm{X}$ & & & & $\mathrm{X}$ & & 3 \\
\hline downloads & $\mathrm{X}$ & & & & $\mathrm{X}$ & & & & 2 \\
\hline flashes & & & & & $\mathrm{X}$ & $\mathrm{X}$ & & & 2 \\
\hline lessons learned & & $\mathrm{X}$ & & & & & $\mathrm{X}$ & & 2 \\
\hline product pages & & & & & & & $\mathrm{X}$ & $\mathrm{X}$ & 2 \\
\hline roadmaps & & & $\mathrm{X}$ & & & & $\mathrm{X}$ & & 2 \\
\hline templates & & $\mathrm{X}$ & & & & & $\mathrm{X}$ & & 2 \\
\hline tools & & & $X$ & & & & $\mathrm{X}$ & & 2 \\
\hline
\end{tabular}

*Genres are listed in order of decreasing frequency of occurrence across sources; genres selected for the final taxonomy are marked in bold.

For each of the sixteen genres, a definition was developed based on descriptions used in the repositories and in web-based dictionaries ${ }^{4}$. Approximately twenty examples of each genre from different sources were then analyzed to identify characteristic features of each with respect to purpose, form, content (style and subject matter), and related genres (Table 5-6).

\footnotetext{
${ }^{4}$ www.onelook.com
} 
Table 5-6: Genre Taxonomy

Class and Definition

Best Practice:

description of a proven methodology or technique for achieving a desired result, often based on practical experience

\section{Cookbook:}

Step-by-step description of how to implement a particular technology or process.

\section{Identifying Features}

Purpose: instructional, advice

Form: many formats, variable length

Style: use of imperatives (you, do, should), term "best

practices" will often appear

Topic: technology, business

Related Genres: cookbook, design pattern, documentation,

technical article, whitepaper

Purpose: demonstrate, procedural

Form: steps are numbered or bulleted; includes examples

and/or templates, screenshots and diagrams; length varies

Style: mix of formal and point form, imperatives, and

sequencing terms (i.e. do this next): common phrases: "how-to",

"click", "step-by-step"

Topic: product focus

Related genres: documentation, manual, technical article - any

of these may contain cookbook sections

Purpose: demonstrate, instruct, sell

Form: demo is often zipped; linked to from html page

Style: lots of recurring terms: demo, filesize, runtime,

download, etc.

Topic: product focus

Related Genres: demo is accessible through product page

Purpose: recommend, illustrate, educate

Form: contains figures and accompanying text

Style: high level, generic terms

Topic: architecture and business/services

Related Genres: technical articles, presentations, technotes, documentation, which may contain design pattern as sub-genre

Purpose: ask and answer questions, provide reference

Form: brief text, links, thread connection

Style: informal and conversational, includes terms: send, I

subject, from, date, etc.

Topic: technical, product

Related Genres: FAQ

Purpose: procedural, instructional, factual

Form: web-based genre (html), short text pieces with lot s of sections and lots of links

Style: imperative

Topic: products

Related Genres: cookbook, manual (documentation, technical article)

Purpose: reporting, documenting

Form: doc. or pdf format, varies in length, report style with headings and sections.

Style: Semi-formal language, technical, product and business

terms, past tense, descriptive

Topic: technical solutions, scenarios, and services

Related Genres:

Purpose: informative, factual

Form: web genre, commonly HTML, often uses internal links, series of short paragraphs, textual

Style: full sentences, question dialogue, common terms are 


\section{Manual:}

book-length document containing practical instructions, rules, and/or steps for performing a task or using some product or technology

\section{Presentation:}

the charts used to accompany a talk or class lecture. “faq”, “question”, “answer”, interrogative pronouns

Topic: technical or product related

Related Genres: product pages, documentation, technotes

Purpose: instructional, documentation

Form: broken down into sections, contains many sub-genres

Style: formal technical style, imperatives

Topic: products or technology

Related Genres: documentation, cookbook

Purpose: instruction, demonstrate, reporting, reviews

Form: format - ppt or pdf

Style: point form, Terms: personal pronouns, I, we , session, presentation, thank you

Topic: new technologies and products

Related Genres: content may be like: best practices, tutorial, design pattern, demo

Problem Report:

record of a reported technical problem together with the details of the customersupport interaction and the steps taken by technical support to resolve it

\section{Product Webpage:}

a webpage designed to provide basic information and links with respect to a particular software product

Purpose: document, log email messages

Style: very informal, messy

Topic: product related, technical solutions Related Genres:

Purpose: factual, marketing

Form: web genre - html

Style: point form, lots of specs (numbers); Terms: features,, overview, product
Form: long unstructured list of appended comments, snippets of

Topic: product features

Related Genres: demo, FAQ, documentation could be subgenres

Purpose: analysis, solving, explaining, documenting

Form: highly structured text, title, abstract, author, references

Style: formal language, instructional, passive

Topic: technical and conceptual

Related Genres: Whitepaper similar; cookbook, best practices, design patterns are subgenres

Purpose: inform, instruct

Form: short (one page),

Style: sentences and point form, lots of specs and numbers, includes sample code or commands

Topic: often product related, low level technical focus

Related Genres: technical article, faq, cookbook

Purpose: instructional, demonstrating

Form: usually zipped files, or collections: classification is done on lead pages

Style: common terms: exercise, lab, pre-requisite, tutorial (often in URL)

Topic: technology

Related Genres: documentation, cookbook

Purpose: instructive and informative (indepth)

Whitepaper:

An authoritative report on a topic in technology containing advanced technical details and guidance.
Form: often pdf or doc format, table of contents, titled and numbered sections, references, executive summary. Usually more than 10 pages and can be over 100 .

Style: formal text

Topic: Mainly products, some technology

Related Genres: technical article 


\section{i. Assessing the Coverage}

Given that the taxonomy represents a subset of all genres in this domain, it was important to assess the coverage of the taxonomy: the portion of documents within the information environment that would fall into one or more of the sixteen categories. This was done by collecting a sample of documents and using the taxonomy as a guide for the manual classification of the sample.

Collecting the sample: In order to obtain a sample of the types of documents likely to be retrieved by the study population, we set up a version of the Multi-Text search engine to crawl the workplace information environment. The search engine crawled and indexed approximately eight gigabytes of documents from sites and repositories in heavy use by the study population, based on a customized set of seed URLs. A set of fifteen queries (Table 5-7) were developed based on questions posed in online discussion groups used by the target population. These queries were designed to represent a range of work tasks and information goals, as indicated in the two right hand columns in Table 5-7. These queries were submitted to the Multi-Text search engine and the first 30 documents from each query were collected.

Table 5-7: Queries used to collect Document Sample

\begin{tabular}{l|l|l}
\multicolumn{1}{c|}{ Query as submitted to system } & \multicolumn{1}{c}{$\begin{array}{c}\text { Associated } \\
\text { Information task }\end{array}$} & \multicolumn{1}{|c}{$\begin{array}{c}\text { Associated } \\
\text { Work Task }\end{array}$} \\
\hline Migration plan J2EE & Decision & Migration \\
\hline Enterprise service bus patterns & Decision & Design \\
\hline VPN SSL/TLS & Decision & Design \\
\hline Container managed relationships JVM & Factual & Implementation \\
\hline Supported JDBC drivers websphere & Factual & Integration \\
\hline Reuse connection property WAS & Factual & Installation/configuration \\
\hline Websphere portal capacity sizing & How to & Architecture \\
\hline Set fetch size method & How to & Performance tuning \\
\hline Websphere commerce engagement template & How to & Project management \\
\hline Scope management & How to & Project management \\
\hline Nodeagent process & Learn & Deployment \\
\hline Dynacache performance & Learn & Performance tuning \\
\hline $\begin{array}{l}\text { Connection pool orphan_timeout } \\
\text { idle_timeout }\end{array}$ & Solve problem & Troubleshooting \\
\hline Wsadmin port plugin & Solve problem & Troubleshooting \\
\hline WAS garbage collection too frequent & Solve problem & troubleshooting \\
\hline
\end{tabular}


Analysis: A substantial number of duplicate and very similar documents were identified and manually removed from the set. The remaining 275 distinct pages were manually classified using the genre taxonomy (Table 5-6), and allowing for multiple classifications of documents. Due to the time involved and the need to protect confidentiality of the documents, classification was carried out by a single researcher.

Results: Based on this assessment, the genre taxonomy accounted for over $75 \%$ of the documents in the set, and only one class, demo, was not represented in the results (Table 5-8). Overall, there was an overlap of about $20 \%$ among the genre classes, due to documents belonging to more than one class.

Table 5-8: Distribution of Results Classified by Genre Taxonomy

\begin{tabular}{l|l|l} 
Genre Class & $\begin{array}{l}\text { \# docs } \\
\text { per class }\end{array}$ & $\begin{array}{l}\text { \% of total } \\
\text { docs in class }\end{array}$ \\
\hline not classified & 63 & 22.9 \\
\hline product documentation & 69 & 25.1 \\
\hline cookbook & 37 & 13.5 \\
\hline technical article & 36 & 13.1 \\
\hline discussion threads & 25 & 9.1 \\
\hline manual & 20 & 7.3 \\
\hline presentation & 17 & 6.2 \\
\hline design pattern & 15 & 5.5 \\
\hline Best practice & 11 & 4.0 \\
\hline FAQ & 10 & 3.6 \\
\hline product page & 10 & 3.6 \\
\hline whitepaper & 9 & 3.3 \\
\hline technote & 4 & 1.5 \\
\hline tutorial & 4 & 1.5 \\
\hline engagement summary & 3 & 1.1 \\
\hline problem report & 1 & 0.4 \\
\hline demo & 0 & 0 \\
\hline Total & 334 & 121.7 \\
\hline & &
\end{tabular}

\subsubsection{Summary of Taxonomy Development}

A bottom-up approach was used to develop taxonomies of tasks and genres for this domain in response to Research Question 3. The taxonomies include eleven broad software engineering work tasks, five generic information tasks, and sixteen genres and definitions for each. Testing of 
the genre taxonomy indicates that it can be used to classify the majority of documents in heavy use by this group. The taxonomies are not meant to be exhaustive, but rather to serve as operational tools to classify tasks and genres that are prevalent in this workplace environment.

\subsection{Validation Survey}

The purpose of this study was to validate and extend the findings from the previous studies, by gathering data directly from a larger sample of the user population. The objectives were to identify the characteristics that differentiate work tasks in this domain and to gather further evidence of associations between tasks and genres. Specifically, the survey collected quantitative user perception data on work task characteristics and the usefulness of genres for information tasks.

\subsubsection{Method}

\section{i. Participants and Recruitment}

The validation study was conducted through a web-based questionnaire posted on the corporate intranet. As with the workplace domain study, recruitment was a serious challenge. To encourage participation, several consultants from the study population assisted with the design of the study and helped to prepare a recruitment presentation. We presented the search study through an open conference call that was advertised by email to the entire consulting group (1000+ members).

The call was sponsored and promoted on team listservs (Appendix 4). However, efforts met with very limited success. Most participants were eventually recruited via emails sent by managers of different services groups in North America and Europe to their team members, as well as personal appeals to colleagues by our contacts within the company. Participants who completed the survey were offered the chance to enter a draw for a small gift.

The final sample consisted of 20 software services consultants. The demographic profile of the group is similar to that of the interview participants, and three of the 20 indicated that they had 
contributed to previous phases of the study. Participants' ages ranged fairly evenly from 26 to 55, with one participant older than 55. Two of the 20 were women. All but one participant held university degrees, of which eight were at the graduate level. The degrees represent a range of disciplines: computer science (seven), computer engineering (three), information systems (three), other sciences (four) and social science (two). All were highly skilled consultants from employment levels seven to 10 (the highest level); they had on average just over seven years of experience in their current work.

\section{ii. Procedure and Instrument}

The questionnaire (Appendix 5) was kept brief in order in to encourage participation. It consisted of four sections.

Section one asked for standard demographic data in order to control for effects of age, gender, seniority and experience.

Section two asked for feedback about the frequency of use of a list of information sources as a means of determining what sources should be covered by the search system.

Section three was designed to identify differences and similarities between the work tasks in the taxonomy, based on characteristics that emerged from the earlier workplace study. Survey participants were given a list of the eleven work tasks together with a brief description, and for each task were asked to indicate their level of agreement on a five point Likert scale with eight descriptive statements, as follows:

- This is a high level activity

- This is a technology-centric activity

- This is a product-centric activity

- This involves a lot of interaction with customers

- This requires on-the-job experience to do well

- This requires in-depth knowledge to do well

- This is a complex activity

- This can employ standard methods 
Section four was designed to identify relationships between information tasks and specific genres. Participants were asked to rate the usefulness of seventeen different genres with respect to five different information tasks, on a five-point Likert scale (not useful to very useful) ${ }^{5}$. An earlier version of the survey asked participants to rate genres with respect to each of the work tasks as well, but this question proved too onerous and time-consuming and was not included in the final survey.

The survey was reviewed by several members of the target population as well as other software engineers and revised to ensure that the terminology was clear. It was posted for a period of three weeks. Potential participants were sent an email with the URL of the questionnaire. Participants were asked to submit their email address as indication of informed consent. The email addresses were stored separately from the data so that responses could not be connected to individual participants. Participants were then provided with the questionnaire. Upon submitting their responses, they were presented with the option of participating in a prize draw. The final page thanked the participants and provided them with the URL of the project page so they could follow the progress of the project. The whole procedure took about fifteen minutes on average.

\section{iii. Data Analysis}

Data was collected into a DB2 database from the web questionnaire and exported into SPSS when the study was complete. The data on work task characteristics was analyzed using ANOVA to identify significant differences between work tasks and correlations were used to identify related characteristics. ANOVA was used to identify significant differences of usefulness scores among genres for each information task.

\footnotetext{
${ }^{5}$ The genre categories used in the survey are slightly different than those in the genre taxonomy, as they were based on an earlier version of the taxonomy.
} 


\subsubsection{Results}

\section{i. Work Task Characteristics - ANOVA}

In section three of the survey, participants indicated their level of agreement with a set of 8 statements about characteristics for each of the 11 work tasks. An ANOVA conducted on the work task characteristics found significant differences between work tasks for all characteristics except for "requires on-the-job experience" which received high scores across all tasks (Table 59). Although differences between tasks were found for most of the characteristics, post hoc testing using Tamhane T2 (for unequal variances) indicated that much of the variation is caused by a small number of tasks, namely project management, architecture and design, which have a similar profile, but differ from the other tasks on a number of characteristics.

Table 5-9: Mean scores and significance tests for characteristics across all tasks

\begin{tabular}{|c|c|c|c|c|c|c|c|c|c|c|c|c|}
\hline $\begin{array}{l}\text { Task } \\
\text { Characteristic }\end{array}$ & 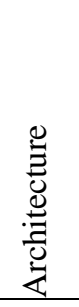 & 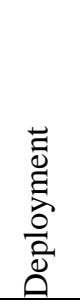 & 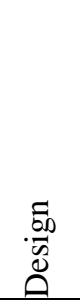 & 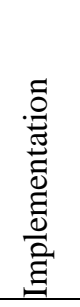 & 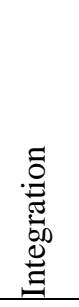 & 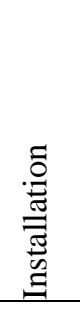 & 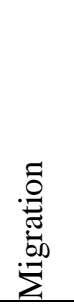 & 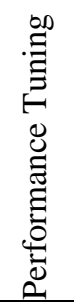 & 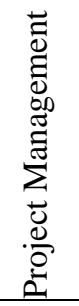 & 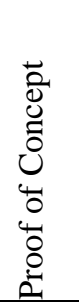 & 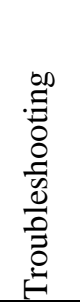 & $\begin{array}{l}\text { Statistical } \\
\text { Significance }\end{array}$ \\
\hline High-level & 3.85 & 2.20 & 3.35 & 2.14 & 2.95 & 1.95 & 2.15 & 2.20 & 3.83 & 2.68 & 2.26 & $\begin{array}{l}F(10,206)=6.660 \\
p<.001\end{array}$ \\
\hline Technology & 3.20 & 4.20 & 3.75 & 4.43 & 4.10 & 4.05 & 3.90 & 3.75 & 2.00 & 4.16 & 4.00 & $\begin{array}{l}\mathrm{F}(10,205)=7.638, \\
\mathrm{p}<.001\end{array}$ \\
\hline Product & 2.65 & 4.25 & 3.25 & 4.43 & 4.20 & 4.75 & 4.72 & 4.65 & 2.00 & 4.63 & 4.37 & $\begin{array}{l}\mathrm{F}(10,204)=21.132, \\
\mathrm{p}<.001\end{array}$ \\
\hline $\begin{array}{l}\text { Customer } \\
\text { Interaction }\end{array}$ & 4.10 & 3.40 & 3.80 & 3.29 & 4.00 & 3.35 & 3.63 & 3.60 & 4.72 & 4.26 & 3.68 & $\begin{array}{l}F(10,205)=3.704 \\
p<.001\end{array}$ \\
\hline Experience & 4.45 & 4.30 & 4.50 & 4.29 & 4.30 & 4.05 & 4.15 & 4.55 & 4.35 & 4.38 & 4.63 & ns \\
\hline Knowledge & 4.27 & 4.07 & 4.60 & 4.63 & 3.87 & 3.87 & 4.27 & 4.36 & 3.15 & 4.50 & 4.79 & $\begin{array}{l}\mathrm{F}(10,150)=4.255 \\
\mathrm{p}<.001\end{array}$ \\
\hline Complexity & 4.40 & 3.95 & 4.45 & 4.24 & 4.55 & 3.85 & 4.25 & 4.30 & 3.50 & 4.16 & 4.58 & $\begin{array}{l}F(10,206)=3.396 \\
p<.001\end{array}$ \\
\hline Standards & 3.90 & 3.85 & 4.20 & 4.24 & 3.45 & 3.85 & 3.35 & 3.75 & 4.33 & 3.47 & 3.47 & $\begin{array}{l}F(10,206)=2.076 \\
p<.05\end{array}$ \\
\hline
\end{tabular}

Correlations based on Spearman's Rho (SPSS output in Appendix 6) indicate relationships among these characteristics. High Level is positively correlated with Customer Interaction (.318) and 
Standards (.194). Product-centred and Technology-centred attributes are strongly correlated (.645) and both are positively correlated with Knowledge $(.173, .210)$ and negatively correlated with High Level (-.364, -.292), suggesting that they are associated with low level tasks that are knowledge intensive. Knowledge and Experience are strongly correlated (.682) and both are strongly correlated with Complexity $(.756, .634)$, but there are no correlations between these three characteristics and High Level. This preliminary exploration of work task characteristics suggests some broader task groupings common to this domain:

- Work Tasks which are high-level, employ standards, involve customer interaction and are less focused on products and technology (e.g. project management, design)

- Work Tasks which are low level, focused on products and technology and are knowledge-intensive (e.g. implementation, migration)

- Work Tasks which are high or low level, very complex and require knowledge and experience to perform (e.g. troubleshooting, performance tuning, integration)

\section{ii. Genres and Information Tasks - ANOVA}

The final section of the questionnaire collected participants' assessments of the usefulness of specific genres for five information tasks, based on a five-point Likert scale. An ANOVA conducted on the genre scores by information task showed significant between group differences for all information tasks (Table 5-10), which provides further support for the hypothesis that task and genre are not independent.

Overall, mean usefulness scores vary from 2.05 at the lowest (demos for problem solving) to 4.74 at the highest (product documents for fact finding). The variation in mean scores is greatest for the fact finding task (2.11 to 4.74 ), and least for learning (2.80 to 4.30 ). 
Table 5-10: Mean scores and significance tests for genres by information tasks

\begin{tabular}{l|l|l|l|l|l} 
Genres & Facts & Decision & How-To & Solve Problem & Learn \\
\hline $\begin{array}{l}\text { Design } \\
\text { documents }\end{array}$ & 3.63 & 3.32 & 2.83 & 2.95 & 2.80 \\
\hline Best practices & 2.58 & 4.58 & 4.11 & 3.74 & 3.75 \\
\hline Demos & 2.11 & 2.47 & 2.94 & 2.05 & 3.80 \\
\hline $\begin{array}{l}\text { Engagement } \\
\text { summaries }\end{array}$ & 2.16 & 3.58 & 2.39 & 3.47 & 2.85 \\
\hline FAQs & 3.33 & 3.42 & 3.67 & 4.00 & 3.80 \\
\hline Cookbooks & 2.95 & 3.58 & 4.67 & 3.58 & 4.10 \\
\hline Newsgroup & 3.11 & 3.63 & 3.67 & 4.00 & 3.05 \\
\hline $\begin{array}{l}\text { Method } \\
\text { documents }\end{array}$ & 2.47 & 3.11 & 3.22 & 2.63 & 2.80 \\
\hline Presentation & 2.74 & 3.32 & 3.11 & 2.58 & 3.85 \\
\hline $\begin{array}{l}\text { Product } \\
\text { documentation }\end{array}$ & 4.74 & 3.74 & 3.94 & 4.00 & 4.10 \\
\hline Manuals & 3.89 & 3.95 & 4.44 & 3.74 & 4.30 \\
\hline Reusable assets & 3.16 & 3.37 & 3.56 & 3.32 & 3.25 \\
\hline Solutions & 2.42 & 3.74 & 3.17 & 3.00 & 3.16 \\
\hline $\begin{array}{l}\text { Technical } \\
\text { articles }\end{array}$ & 3.95 & 3.79 & 3.78 & 3.47 & 3.85 \\
\hline Technotes & 4.00 & 3.16 & 3.50 & 4.05 & 3.30 \\
\hline Tutorials & 2.53 & 2.84 & 3.39 & 2.37 & 3.85 \\
\hline whitepapers & 2.89 & 3.53 & 3.11 & 2.95 & 3.84 \\
\hline & & & & & $\mathrm{F}(16,321)$ \\
\hline Significance & $\begin{array}{l}\mathrm{F}(16,304) \\
=7.178\end{array}$ & $\mathrm{~F}(16,306)$ & $=3.154 \mathrm{p}<.001$ & $\mathrm{~F}(16,287)$ \\
$=4.44 \mathrm{p}<.001$ & $=4.541 \mathrm{p}<.001$ & $=4.808 \mathrm{p}<.001$ \\
\hline & $\mathrm{p}<.001$ & & & & \\
\hline
\end{tabular}

Table 5-11 shows the genres divided into three bins, based on mean usefulness scores. For each task, there are a small number of genres with mean scores in the upper range (4-5), which are most likely to be useful, a larger number of genres in the middle range (3-3.9), which may or may not be useful, and a somewhat smaller number in the lower range (2-2.9), which are less likely to be useful. For each task, a different genre received the highest mean score. These are genres which are clearly aligned with the tasks in terms of purpose: best practice documents are explicitly designed to support decision-making, cookbooks to explain how to do something, product documents to look up facts, technotes to solve problems, and manuals to support learning. Genres are also distributed differently for each task. Finding facts is positively associated with a small number of genres, but negatively associated with a relatively large number. Solving problems is positively associated with more genres than any other task, but is also negatively 
associated with a large number. The remaining tasks are positively and negatively associated with small numbers of genres, with most genres in the middle, "neutral” range.

Table 5-11: Mean Genre Scores by Task

\begin{tabular}{l|l|l|l}
$\begin{array}{l}\text { Information } \\
\text { Task }\end{array}$ & Upper Range (4.0-4.9) & Middle Range (3.0-3.9) & Lower Range (2.0-2.9) \\
\hline Finding Facts & $\begin{array}{l}\text { product docs (4.74) } \\
\text { technotes (4.0) }\end{array}$ & Remaining six genres & $\begin{array}{l}\text { cookbooks (2.95) } \\
\text { whitepapers (2.89) } \\
\text { presentation (2.74) } \\
\text { best practices (2.58) } \\
\text { tutorials (2.53) } \\
\text { methods (2.47) } \\
\text { solutions (2.42) } \\
\text { demos (2.11) } \\
\text { engagement materials (2.16) }\end{array}$ \\
\hline Making & best practices (4.58) & Remaining 14 genres & $\begin{array}{l}\text { tutorials (2.84) } \\
\text { demos (2.47) }\end{array}$ \\
\hline Decisions & $\begin{array}{l}\text { Cookbooks (4.67) } \\
\text { manuals (4.44) } \\
\text { best practices (4.11) }\end{array}$ & Remaining 11 genres & $\begin{array}{l}\text { design docs (2.83) } \\
\text { engagement materials (2.39) } \\
\text { demos (2.94) }\end{array}$ \\
\hline Solve Problem & $\begin{array}{l}\text { technotes (4.05) } \\
\text { Faqs (4.00) } \\
\text { Newsgroups (4.00) } \\
\text { product docs (4.00) }\end{array}$ & Remaining seven genres & $\begin{array}{l}\text { design docs (2.95) } \\
\text { whitepaper (2.95) } \\
\text { methods (2.63) } \\
\text { presentations (2.58) } \\
\text { tutorials (2.37) } \\
\text { demos (2.05) }\end{array}$ \\
\hline Learn & $\begin{array}{l}\text { Manuals (4.30) } \\
\text { product docs (4.10) } \\
\text { cookbooks (4.10) }\end{array}$ & Remaining 11 genres & $\begin{array}{l}\text { design docs (2.80) } \\
\text { engagement mats (2.85) } \\
\text { methods (2.80) }\end{array}$ \\
\hline & & & \\
\hline
\end{tabular}

There are also groupings within the genres. Some genres are strongly associated with most tasks

(e.g. manuals, product documents, technical articles, best practices); some have no strong positive associations (e.g. design docs, engagement materials, methods); and some are strongly associated with only one or two tasks (e.g. presentations, technotes, newsgroups, demos, cookbooks).

\subsubsection{Summary of Validation Survey}

Results of the validation questionnaire provide additional support for the hypothesis that task and genre are dependent, specifically with respect to information tasks. Usefulness scores assigned to 17 genres varied significantly with respect to each of the five tasks. Based on the mean scores for each genre, it was possible to identify genres which are more or less likely to be useful for each of 
the information tasks. The survey also clarifies the nature of work tasks in this domain. Experience is an important requirement for all work tasks, but there is significant variation between work tasks based on other characteristics, notably level of abstraction and complexity. Results point to a framework for categorizing software engineering work tasks and genres, which has potential for generalizing applications of Task-Genre relationships. The major limitations of this study are the small sample size and the relative homogeneity of responses obtained using the five point Likert scale. Nevertheless, results do offer some validation of previous findings as well as opening up avenues for further exploration of work task characteristics.

\subsection{Summary}

This chapter reported on a series of studies designed to confirm, characterize and operationalize the task - genre relationship in this workplace domain. The first section described an analysis of metadata used in a document repository focussing on co-occurrence patterns between Task and Genre metatags. The second section described the methods and analysis used to develop domainspecific taxonomies of tasks and genre categories. The final section reported on a survey conducted to validate and extend previous findings with respect to the nature of work tasks and the relationship between information tasks and genres.

Results of these studies confirm the existence of a relationship between these factors, provide input for the development of a matrix of associations between the categories of the task and genre taxonomies, and suggest ways in which the task-genre model can be generalized. These studies paved the way for the next phase of the research, which involved the implementation and evaluation of task-genre relationships in the framework of a search system. 


\section{Implementing Task-Genre Relationships in Search}

\subsection{Overview}

The third phase of this research involved implementing task-genre relationships within a search system, which is the focus of this chapter, and evaluating the implementation, which is the focus of Chapter 7. The X-Site search system was designed and implemented in the study setting in order to test the practical value of making use of task-genre relationships in a real-life work environment. Building the system also provided the opportunity to explore the implementation issues associated with making use of task and genre context in an enterprise information environment.

X-Site was implemented as an extension to MultiText, a pre-existing indexing and retrieval engine developed at the University of Waterloo. This chapter describes the design rationale for the system, focusing on the components developed in the course of this research. These include a custom search interface, a genre classifier, and a set of task-genre associations which drive a search results filter. Detailed information on the crawling, indexing and ranking components of the system are not presented here, as these were developed for the MultiText system, outside the 
framework of this research. Documentation for MultiText can be found elsewhere (Clarke, Cormack, \& Burkowski, 1994).

\subsection{System Overview}

$\mathrm{X}$-Site indexes documents and Webpages from a range of internal corporate repositories and public Websites and provides a single point of access to these resources. The retrieval mechanism aims to identify documents which are both topically relevant and useful to the task at hand. Topically relevant documents are identified and ranked using the traditional text retrieval approach of combining a number of measures of similarity between the text of the query and the text of the documents in the collection. For purposes of this research, the existing retrieval algorithm within MultiText was used to determine topical relevance. Useful documents are identified based on known positive associations between document genres and search task profiles, based on evidence of these associations gathered in previous phases of this research. Usefulness is operationalized as a binary measure and implemented as a filter that removes from the set of results all document genres that are not positively associated with the task profile. A conceptual diagram of the $\mathrm{X}$-Site system illustrating this two-step ranking and filtering function is shown in Figure 6-1.

Figure 6-1: X-Site search system components

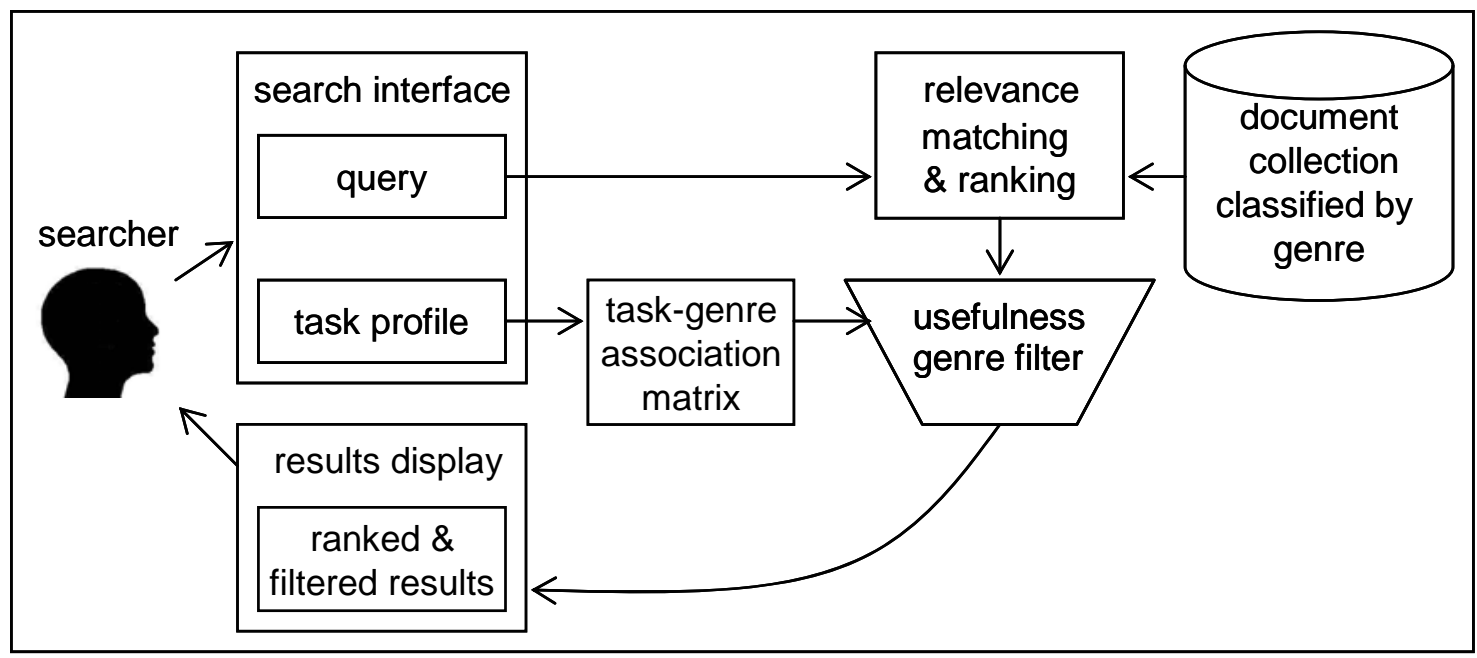


The system works as follows. The document collection is automatically classified by genre as part of the index-building process. At run-time, a searcher submits a query together with a task profile, consisting of one information task and one work task selected from the task taxonomies. For example, a searcher may issue the keyword query "JVM garbage collection" together with the task profile: "Learn about” and "Performance Tuning”. The query is used to retrieve and rank a set of results using statistical methods based on the vector-space retrieval model. The task profile is used to identify an appropriate genre filter based on a static task-genre association matrix. The genre filter then identifies all documents classified as genres which are positively associated with the task profile and removes all others from the results set. The filtered set of results is displayed to the searcher.

$\mathrm{X}$-Site makes use of three contextual components in addition to the basic search engine functionality:

- the genre classifier, which uses machine learning methods to pre-process and tag the document collection by genre as part of the indexing process, prior to runtime;

- the task profile, which is composed of a work task and an information task elicited from the searcher at runtime;

- the task-genre association matrix (TGAM), which is a static component specifying the relationships between the task taxonomies and genre taxonomies.

The sections below will describe the design and implementation of each of these components and provide a description of the X-Site user interface.

\subsubsection{Genre Classifier}

Although genre was identified as a key component of searching and selecting behaviour in this research, there are no standard genre or metadata taxonomies in use in this information 
environment, and any genre tagging that has been done is too sparse and inconsistent to be of use. In practical terms, with no consistent standards and no centralized control over the creation and publication of documents, the only way to classify the collection was to use automatic, post-hoc methods.

Automatic genre classification is an active area of research, but operational systems that employ genre classification have not yet been developed. So this component is novel and experimental. X-Site makes use of SVM Light, a readily available open source support vector machine (SVM) classification package (Joachims, 1998, 1999). SVMs have been shown to perform well for genre classification in comparison with other methods, including Naïve Bayes, C4.5 decision trees (Dewdney, VanEss-Dykema, \& MacMillan, 2001) and neural networks (Meyer zu Eissen \& Stein, 2004), and are well-suited to text classification (Joachims, 1998). An SVM classifier uses a set of examples to train separate classifiers for each class. These classifiers run independently over the whole collection, so that each object can be assigned to multiple categories or to none. This approach is well-suited to our genre taxonomy, which does not assume that the categories are mutually exclusive or exhaustive.

To train the classifiers, sets of fifty examples of each genre in the taxonomy were manually collected from websites, databases and document repositories used by the target population. Each document in the training set was initially assigned to a single class. In order to avoid over-training of the classifiers to specific document templates that exist within different repositories, examples were taken from a wide range ( $>50$ ) of different locations (servers) within the organizational information space. Feature selection from the training data used a "bag of words" approach in which all text features were included. No structural document features were included, due to the challenge of extracting these from documents in a wide range of file formats. Word stemming, which is common in subject-based classification, was not used as there is insufficient evidence to suggest that word stems are more expressive of genre than inflected terms (Muresan, Smith, Cole, 
Liu, \& Belkin, 2006). SVM Light uses tuning parameters to set the rate of error tolerance and the relative weight of positive versus negative examples in the training set. In lieu of any guidelines on setting these parameters in this type of application, trial and error was used and parameters were set uniformly for all genre classes. Tests on pre-classified examples were used to tune the parameters. Table 6-1 provides the results obtained from leave-one-out cross validation tests on the training data from two runs. Results from Run 1 were based on a relatively high error tolerance and a heavy weight in favour of positive examples. Using these settings, high levels of recall were achieved, but the cost in terms of precision meant that documents were over-classified and assigned to multiple classes ${ }^{6}$.

Table 6-1: Precision Recall Estimates on Training Data

\begin{tabular}{l||l|l||l|l}
\multirow{2}{*}{ Genres } & \multicolumn{2}{|l||}{ Run 1 } & Run 2 & \\
\cline { 2 - 4 } best practice & \% recall & \% precision & \% recall & \% precision \\
\hline cookbook & 90 & 19 & 64 & 49 \\
\hline demos & 97 & 22 & 83 & 41 \\
\hline design patterns & 91 & 94 & 82 & 100 \\
\hline discussion thread & 89 & 54 & 74 & 82 \\
\hline documentation & 97 & 74 & 94 & 94 \\
\hline engagement summary & 90 & 39 & 63 & 60 \\
\hline faq & 83 & 52 & 73 & 82 \\
\hline manual & 99 & 13 & 65 & 96 \\
\hline presentation & 98 & 71 & 98 & 76 \\
\hline problem report & 88 & 31 & 66 & 72 \\
\hline product page & 97 & 81 & 96 & 91 \\
\hline technical article & 96 & 68 & 92 & 92 \\
\hline technotes & 96 & 21 & 84 & 48 \\
\hline tutorial & 81 & 36 & 63 & 68 \\
\hline whitepaper & 84 & 83 & 78 & 93 \\
\hline overall average & 91 & 37 & 79 & 56 \\
\hline
\end{tabular}

As an illustration, the classifier was able to identify $90 \%$ of best practice documents as such, but of all the documents identified as best practices only $19 \%$ actually belonged to that category. This type of classification error would result in a very weak filter, because documents would be

\footnotetext{
${ }^{6}$ Recall refers to the percentage of documents belonging to a class that were correctly assigned to that class. Precision refers to the percentage of documents assigned to a class that actually belong to that class.
} 
assigned to multiple categories, which would increase the likelihood of being positively associated with any given task profile. For Run 2, error tolerance was reduced to achieve a more balanced trade-off between recall and precision, resulting in average values of $79 \%$ recall and 76\% precision. Using these settings, more documents will fail to be classified, but fewer documents will be incorrectly classified. Run 2 settings were implemented in X-Site.

\subsubsection{Task Profile}

The two-dimensional approach to tasks developed in earlier phases of this research was used to establish a task profile, consisting of a work task and an information task. These are explicitly specified by the searcher via the search interface by selecting options from the task taxonomies presented in drop-down lists. The values are shown in Table 6-2.

\section{Table 6-2: Task Taxonomies Values}

\begin{tabular}{l|l} 
Work Tasks & Information Tasks \\
\hline Architecture & Learn about a topic \\
Design & Make a decision \\
Implementation & Find out how to \\
Deployment & Find facts \\
Installation \& Configuration & Find a solution \\
Integration & \\
Migration & \\
Performance Tuning & \\
Troubleshooting & \\
Project Management & \\
Proof of Concept &
\end{tabular}

Relying upon the searcher to describe their own task context makes sense in this type of search environment, since most searches are motivated by work-related activities and the searchers have sufficient domain expertise to be able to describe their own tasks. Furthermore, the workplace domain study showed that this population has a marked natural tendency to describe projects and activities in terms of the underlying tasks. This approach is consistent with Belkin's (1980) ASK theory, which suggests that it is more reasonable to expect searchers to describe their current context (problematic situation) than to describe what they are looking for. However, there is also a wealth of evidence to show that search engine users put very little effort into formulating 
complex queries and almost never use advanced search interfaces (Spink, Jansen, \& Saracevic, 2001); therefore, it was important that task profile specification not be onerous.

In future, the use of implicit measures of user behaviour may be considered as a means of deriving task models without the searchers' explicit participation; however this is beyond the scope of the current work.

\subsubsection{Task-Genre Associations Matrix (TGAM)}

The TGAM (Table 6-3) was developed to serve as a simple representation of relationships between the task and genre taxonomies to guide the genre filter in the X-Site system. Associations are expressed in the matrix as positive (+1), neutral (0) or negative $(-1)$. A positive association means that there is evidence that a given genre tends to be used within a given task context, a negative association indicates the opposite, and a neutral association means that available evidence supports neither a positive nor a negative association.

Associations were determined based on a manual synthesis of evidence of relationships gathered over the course of the research, and were refined based on initial testing of the system. This approach was taken because each of the studies conducted provided some evidence of relationships, but evidence was drawn from different sources, took different forms, and much of it referenced somewhat different genre and task categories. This collection of diverse data could only be integrated manually. 
Table 6-3: Task-Genre Association Matrix

\begin{tabular}{|c|c|c|c|c|c|c|c|c|c|c|c|c|c|c|c|c|}
\hline \multirow[t]{2}{*}{ Genres } & \multicolumn{5}{|c|}{ Information Goals } & \multicolumn{11}{|c|}{ Work Tasks } \\
\hline & 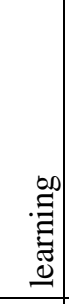 & $\begin{array}{l}0 \\
3 \\
0 \\
\\
\end{array}$ & 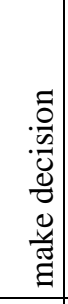 & 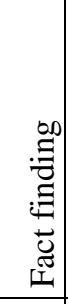 & $\begin{array}{l}\text { E } \\
\frac{0}{0} \\
0 \\
0 \\
0 \\
0 \\
0 \\
0 \\
0\end{array}$ & 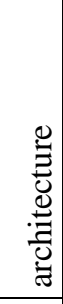 & 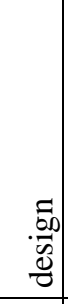 & 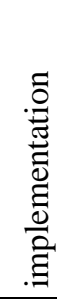 & 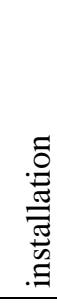 & 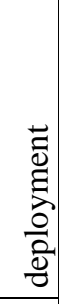 & 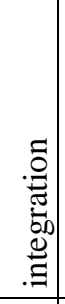 & 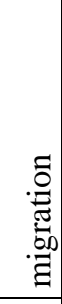 & $\begin{array}{l}u \\
\varrho \\
\end{array}$ & 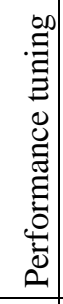 & $\begin{array}{l}0 \\
. \Xi \\
0 \\
0 \\
\frac{7}{0} \\
\frac{0}{0} \\
0 \\
0 \\
\end{array}$ & 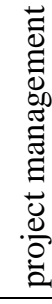 \\
\hline best practices & 0 & 0 & 1 & -1 & 0 & 1 & 1 & 1 & 0 & 1 & 0 & 1 & 0 & 1 & 0 & 1 \\
\hline cookbook & 0 & 1 & 0 & 0 & 0 & -1 & -1 & 0 & 1 & 1 & 0 & 0 & 0 & 0 & 0 & 0 \\
\hline demos & 0 & 0 & -1 & -1 & -1 & -1 & -1 & -1 & 1 & 0 & 0 & 0 & 0 & 1 & 1 & 0 \\
\hline design patterns & 0 & -1 & -1 & 0 & 0 & 1 & 1 & 0 & -1 & 0 & 0 & 0 & 1 & 0 & 0 & 0 \\
\hline discussion threads & -1 & 0 & 1 & 0 & 1 & 0 & 0 & 0 & 0 & 0 & 0 & 0 & 0 & 1 & 1 & 0 \\
\hline documentation & 1 & 1 & 0 & 1 & 0 & 0 & 0 & 0 & 1 & 0 & 0 & 1 & 1 & 0 & 0 & 0 \\
\hline $\begin{array}{l}\text { engagement } \\
\text { summaries }\end{array}$ & -1 & -1 & 0 & -1 & 0 & 1 & 0 & 0 & 0 & 0 & 0 & 1 & 1 & 0 & 0 & 1 \\
\hline FAQs & 0 & 0 & 0 & 0 & 1 & 0 & 0 & 0 & 0 & 0 & 0 & 0 & 0 & 0 & 0 & 0 \\
\hline Manuals & 1 & 1 & 1 & 1 & 0 & 1 & 1 & 1 & 0 & 1 & 0 & 0 & 0 & 0 & 0 & 1 \\
\hline presentation & 0 & -1 & -1 & 0 & -1 & 1 & 1 & 0 & 0 & 0 & 0 & 1 & 0 & 0 & 0 & 1 \\
\hline problem reports & -1 & -1 & 0 & 0 & 1 & 0 & 0 & 0 & 0 & 0 & 0 & 0 & 0 & 0 & 1 & 0 \\
\hline product pages & 1 & 0 & 0 & 1 & 0 & 0 & 0 & 0 & 0 & 0 & 1 & 1 & 1 & 0 & 0 & 0 \\
\hline technical article & 0 & 1 & 0 & 0 & 0 & 0 & 0 & 1 & 0 & 0 & 0 & 1 & 0 & 0 & 0 & 0 \\
\hline technotes & 0 & 0 & -1 & 1 & 1 & 0 & 0 & 0 & 0 & 0 & 1 & 0 & 0 & 1 & 1 & 0 \\
\hline tutorials & 1 & 0 & -1 & -1 & -1 & 0 & 0 & 0 & 0 & 0 & 0 & 0 & 0 & 0 & 0 & 0 \\
\hline whitepapers & 0 & -1 & 0 & 0 & -1 & 1 & 0 & 0 & 0 & 0 & 0 & 0 & 0 & 0 & 0 & 0 \\
\hline
\end{tabular}

Data on the associations between Genres and Information Tasks was available from the

usefulness scores collected in the Validation Survey and was supplemented by partial data

gathered in the interviews and the ICR metadata study. There was considerable agreement across

the studies as to the most useful Genres for each of the five Information Tasks, so redundancy

was used to confirm the existence of positive associations. Low usefulness scores and/or low co-

occurrence frequencies were interpreted as evidence of negative relationships. A summary of this evidence is presented in Table 6-4. 
Table 6-4: Summary of Evidence of Associations for Information Tasks and Genres

\begin{tabular}{|c|c|c|c|}
\hline $\begin{array}{l}\text { Information } \\
\text { Tasks }\end{array}$ & $\begin{array}{l}\text { Validation Survey } \\
\text { (Table 5-11) }\end{array}$ & $\begin{array}{l}\text { Interview and Focus } \\
\text { Groups }\end{array}$ & $\begin{array}{l}\text { ICR Cross Tabulation } \\
\text { (Table 5-2) }\end{array}$ \\
\hline Learning & $\begin{array}{l}\text { ++manuals } \\
\text { +documentation } \\
\text { +cookbooks } \\
\text {-design documents } \\
\text {-engagement materials }\end{array}$ & $\begin{array}{l}\text { +manuals } \\
\text { +documentation } \\
\text { +technical articles } \\
\text { +tutorials }\end{array}$ & $\begin{array}{l}\text { ( Educate) } \\
\text { ++lecture/lab } \\
\text { ++presentation } \\
\text { +reading material } \\
\text {-architecture/ design } \\
\text {-cookbook } \\
\text {-demo } \\
\text {-source code }\end{array}$ \\
\hline How to & $\begin{array}{l}\text { ++cookbooks } \\
\text { +manuals } \\
\text { +best practices } \\
\text {-design documents } \\
\text {-engagement materials }\end{array}$ & $\begin{array}{l}\text { +cookbooks } \\
+ \text { manuals } \\
\text { +documentation } \\
\text { +technical articles }\end{array}$ & $\begin{array}{l}\text { ( Guide, Demonstrate) } \\
\text { ++demo } \\
\text { +reading material } \\
\text { +cookbook } \\
\text { +source code } \\
\text {-presentation } \\
\text {-product feedback }\end{array}$ \\
\hline $\begin{array}{l}\text { Make a } \\
\text { Decision }\end{array}$ & $\begin{array}{l}\text { ++best practices } \\
\text {-tutorials } \\
\text {-demos }\end{array}$ & $\begin{array}{l}\text { +best practices } \\
\text { +technical articles } \\
\text { +white papers } \\
\text { +manuals } \\
\text { +discussion threads }\end{array}$ & \\
\hline Fact Finding & $\begin{array}{l}++ \text { documentation } \\
\text { +technotes } \\
\text {-demos } \\
\text {-engagement materials } \\
\text {-tutorials }\end{array}$ & $\begin{array}{l}\text { +documentation } \\
\text { +technotes } \\
\text { +websites } \\
\text { +manuals } \\
\text { +problem reports }\end{array}$ & $\begin{array}{l}\text { ( reference) } \\
\text { +collection } \\
\text { +legal material } \\
\text { ++schedule } \\
\text { +website } \\
\text {-demo } \\
\text {-presentation }\end{array}$ \\
\hline $\begin{array}{l}\text { Solve } \\
\text { Problem }\end{array}$ & $\begin{array}{l}\text { ++technotes } \\
\text { +FAQs } \\
\text { +discussion threads } \\
\text { +documentation } \\
\text {-demos } \\
\text {-tutorials } \\
\text {-presentations }\end{array}$ & $\begin{array}{l}\text { +technotes } \\
\text { +discussion threads } \\
\text { +problem reports } \\
\text { +technical articles }\end{array}$ & $\begin{array}{l}\text { ( Support) } \\
\text { +reading material } \\
\text {-presentation } \\
\text {-source code }\end{array}$ \\
\hline
\end{tabular}

Evidence of strong positive (++), positive (+) and negative (-) associations

The main sources of evidence for associations between Work Tasks and Genres were the interviews and the ICR metadata study. For some Work Tasks, such as project management and architecture the same positively associated Genres were identified in both studies; however, there was less overlap in the data from different sources for Work Task associations than for Information Tasks, so associations tended to be based on a single source of evidence. Low cooccurrence frequencies were used to infer negative relationships. Evidence is summarized in Table 6-5. 
Table 6-5: Summary of Evidence of Associations for Work Tasks and Genres

\begin{tabular}{|c|c|c|c|}
\hline \multirow{2}{*}{$\begin{array}{l}\text { Work Tasks } \\
\text { Architecture }\end{array}$} & \multirow{2}{*}{$\begin{array}{l}\begin{array}{c}\text { Interview and Focus } \\
\text { Groups }\end{array} \\
\text { +design patterns } \\
\text { +best practices } \\
\text { +manuals } \\
\text { +online courses } \\
\text { +engagement materials } \\
\text { +white papers }\end{array}$} & \multicolumn{2}{|c|}{$\begin{array}{c}\text { ICR Cross Tabulation } \\
\text { (Table 5-2) }\end{array}$} \\
\hline & & $\begin{array}{l}\text { +architecture/design } \\
\text { +presentation }\end{array}$ & $\begin{array}{l}\text {-cookbook } \\
\text {-engagement summary } \\
\text {-lecture/lab } \\
\text {-source code }\end{array}$ \\
\hline Design & $\begin{array}{l}\text { +best practices } \\
\text { +manuals }\end{array}$ & $\begin{array}{l}\text { (Architecture) } \\
\text { +architecture/design } \\
+ \text { presentation }\end{array}$ & $\begin{array}{l}\text {-cookbook } \\
\text {-engagement summary } \\
\text {-lecture/lab } \\
\text {-source code }\end{array}$ \\
\hline Implementation & $\begin{array}{l}\text { +best practices } \\
\text { +manuals } \\
\text { +websites } \\
\text {-demos }\end{array}$ & $\begin{array}{l}\text { +reading materials } \\
\text { +source code }\end{array}$ & -presentation \\
\hline Deployment & $\begin{array}{l}\text { +best practices } \\
\text { +manuals }\end{array}$ & -presentation & \\
\hline $\begin{array}{l}\text { Installation \& } \\
\text { Configuration }\end{array}$ & $\begin{array}{l}\text { +manuals } \\
\text { +documentation }\end{array}$ & $\begin{array}{l}\text { +cookbook } \\
\text { +demo } \\
\text { +source code } \\
\text { +white paper }\end{array}$ & $\begin{array}{l}\text {-architecture/design } \\
\text {-lecture/lab } \\
\text {-presentation }\end{array}$ \\
\hline Integration & $\begin{array}{l}\text { +technotes } \\
\text { +websites }\end{array}$ & $\begin{array}{l}\text { + architecture/design } \\
\text { + cookbook } \\
\text { +source code }\end{array}$ & $\begin{array}{l}\text {-lecture/lab } \\
\text {-presentation }\end{array}$ \\
\hline Migration & $\begin{array}{l}\text { +best practices } \\
\text { +websites } \\
\text { +presentations } \\
\text { +documentation }\end{array}$ & $\begin{array}{l}\text { +engagement summary } \\
\text {-lecture/lab }\end{array}$ & \\
\hline Performance Tuning & $\begin{array}{l}\text { +technotes } \\
\text { +best practices }\end{array}$ & $\begin{array}{l}\text { +demo } \\
\text { +discussion threads }\end{array}$ & -lecture/lab \\
\hline Troubleshooting & $\begin{array}{l}\text { +technical articles } \\
\text { +discussion threads } \\
\text { +technotes } \\
\text { +problem reports }\end{array}$ & $\begin{array}{l}\text { (Debugging) } \\
\text { +demo } \\
\text { +source code } \\
\text { +website }\end{array}$ & $\begin{array}{l}\text {-lecture/lab } \\
\text {-presentation }\end{array}$ \\
\hline Project Management & $\begin{array}{l}\text { +engagement materials } \\
\text { +presentations } \\
\text { +best practices } \\
\text { +manuals }\end{array}$ & +engagement summary & \\
\hline Proof of Concept & $\begin{array}{l}\text { +documentation } \\
\text { +design patterns } \\
\text { +websites }\end{array}$ & $\begin{array}{l}\text { +engagement summary } \\
\text {-presentation }\end{array}$ & \\
\hline
\end{tabular}

The initial version of the TGAM included a large number of both positive and negative associations. However, early testing of the TGAM as implemented in the X-Site system indicated that the many positive associations resulted in a weak filter that had little impact on search results. In addition, it was observed that the negative associations between Genres and Work 
Tasks frequently negated positive associations with Information Tasks, resulting in the loss of useful documents. The final version of the TGAM (Table 6-3) was produced by removing positive associations for which there was less evidence and by removing all but the strongest negative associations between Work Tasks and Genres.

\section{i. Application of the TGAM for Filtering}

The three levels of associations described in the TGAM allow for the genre taxonomy to be mapped onto a two-dimensional space created by each task profile combination. Figure 6-2 shows the genre distribution for the task profile: work task=architecture and information task=finding facts.

Figure 6-2: Distribution of genres for task profile: architecture and fact finding

\begin{tabular}{|c|c|c|c|}
\hline 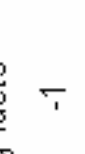 & $\begin{array}{l}\text { best practices } \\
\text { engagement } \\
\text { summary }\end{array}$ & tutorial & \\
\hline$\square$ & $\begin{array}{l}\text { design pattern } \\
\text { presentation } \\
\text { whitepaper }\end{array}$ & $\begin{array}{l}\text { discussion thread } \\
\text { FAQ } \\
\text { problem report } \\
\text { technical article }\end{array}$ & $\begin{array}{l}\text { cookbook } \\
\text { demo }\end{array}$ \\
\hline$\overline{+}$ & manual & $\begin{array}{l}\text { documentation } \\
\text { product page } \\
\text { technote }\end{array}$ & \\
\hline & +1 & $\begin{array}{c}0 \\
\text { ask }=\text { architect }\end{array}$ & -1 \\
\hline
\end{tabular}

Nine possible conditions exist for each genre with respect to the task profile, ranging from a positive association with both types of tasks to a negative association for both. The X-Site genre filter was configured to retrieve genres with a combined value of more than zero, which in this example would include the genres in the shaded cells. Manuals are positively associated with both tasks, and have a combined value of 2; documentation, product pages and technotes have a 
combined value of 1 , based on their association with fact-finding; and design patterns, presentation, and whitepapers, have a combined value of 1 based on their association with architecture. Genres that have a null or negative value for a given task profile are considered to be less likely to be useful, and are not included in the results set. The filter errs on the side of inclusion for documents tagged with multiple genre classes. If any of the genres are positively associated with the task profile, the document is included. Documents in the collection that are not assigned any genre by the automatic classification process are removed from the results set.

The genre filter serves two functions. First, it defines a "genre universe" for this work domain in terms of the 17 genres in the taxonomy, and filters out everything else. This effectively creates an information-rich, domain specific document pool within the general collection. Second, it dynamically subdivides this pool into sets of genres that are likely to be of most use for particular task profiles. The choice to implement the TGAM as a filter rather than as input to the ranking algorithm was based on two considerations. A filter was more likely to show a strong and independent effect of the task-genre relationships in this experimental system. Emphasizing this effect was important, because the contextual associations play a secondary role to the primary topical associations used to rank the document set. Also, filtering requires only binary data on the relationships between tasks and genres. This was relatively easy to determine from the available sources of evidence. A more subtle weighting and ranking implementation of task-genre relationships would require data on the strengths of associations.

\subsubsection{Search Interface}

The X-Site search interface consists of the initial search form (Figure 6-3) and a results display form (Figure 6-4). The design supports interactive searching by using a large query input box and the combined query input/results screen, which allows the searcher to update and re-issue their query terms and task profile directly from the results screen. Search functionality is purposely limited to query input, task profile specification by selection from drop-down lists and 
access to a Help form in order to focus the evaluation on the contribution of task-genre associations.

Figure 6-3: X-Site Query Interface

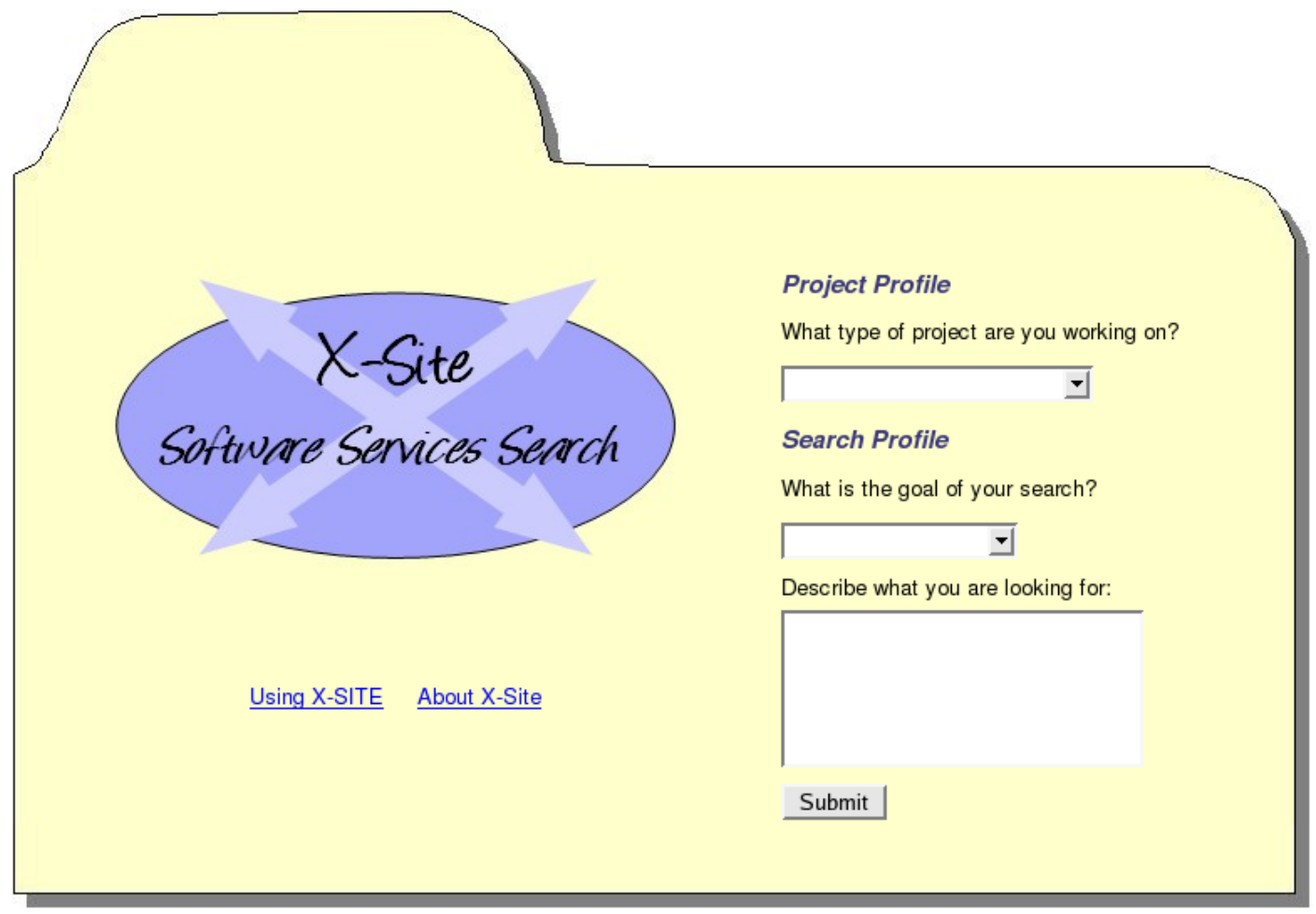

The primary usability concern in designing the interface was to ensure that the novel component - the task profile - made sense to searchers, and was easy to use. A folder design metaphor with "Project Profile” and "Search Profile” labels was used in order to invoke a project-oriented mental model of searching that would be suited to the work practices of consultants. Since consultants commonly use work tasks as way of classifying projects, the list of eleven work tasks is under the heading "Project Profile" and the question: "What type of project are you working on?” The information task and query textboxes are grouped together under the heading "Search Profile”, since they both relate to the specific search situation. Searchers are asked to select the goal of their search (information task) and to describe what they are looking for (query). 
Figure 6-4: X-Site Results Page

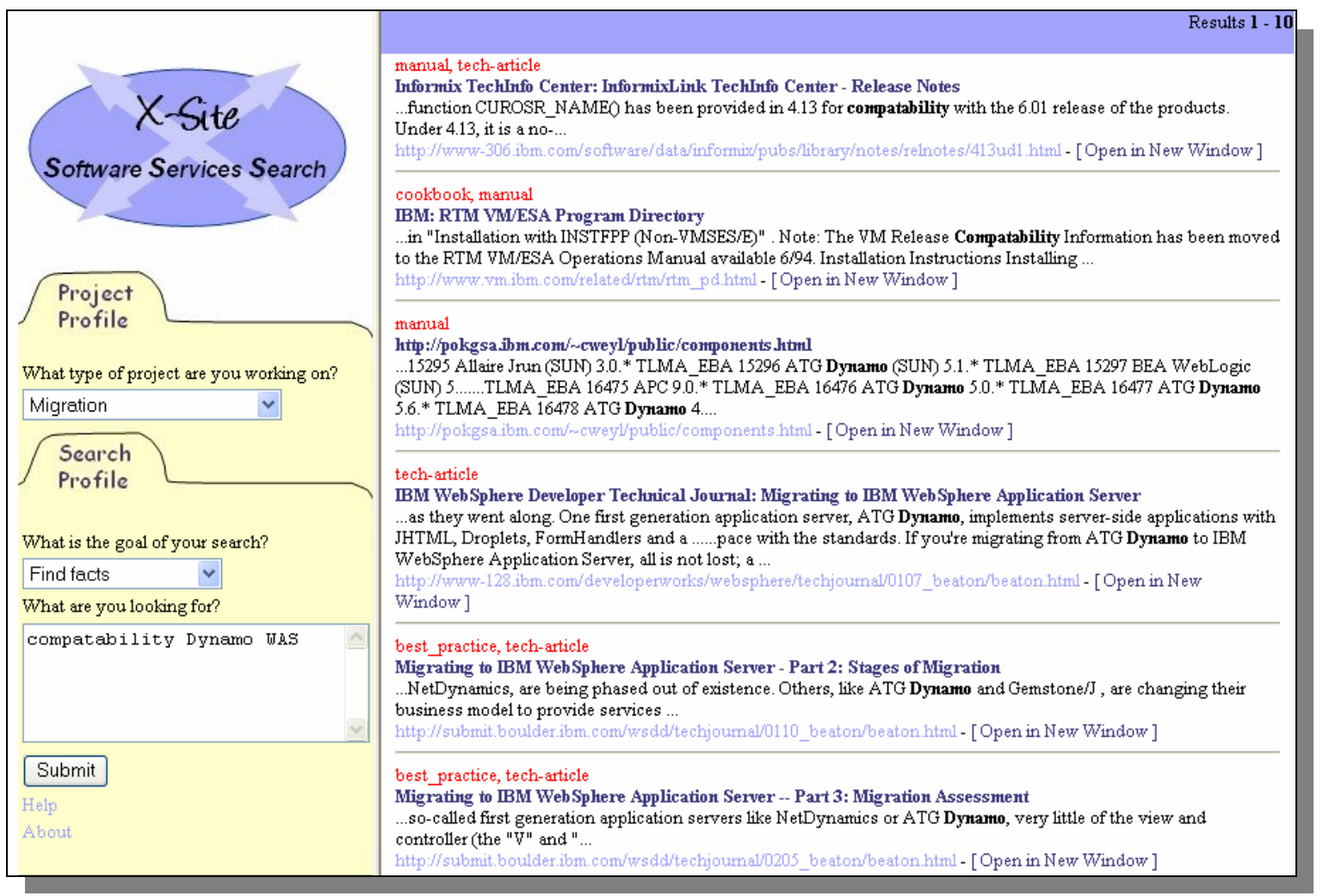

The results screen includes the basic components of most search engine results screens, including a list of the first ten results in the form of a hyperlinked document title, a short snippet from the text showing the keywords in context, and a hyperlinked URL. In addition, if genre classes have been assigned to a document, these genre classes appear in red text above the hitlist entry. As noted above, a miniature version of the opening search screen appears as a sidebar, so that the search input can be updated easily.

\subsection{Summary}

This chapter described the design of the X-Site search system: a prototype enterprise search engine in which task-genre relationships were implemented as a filter. The contextual components of X-Site are: an automatic genre classifier, a task profile elicited from the searcher, a task-genre association matrix and a genre filter. X-Site was designed specifically to evaluate the contribution of task-genre relationships. However, it represents only one possible 
implementation of task-genre relationships, the design of which was constrained by the nature of the available data, and the contingencies of implementation in a real-world setting. Other possible implementations may be considered in further research, either through improvements of the X-Site prototype, or through the adoption of different models to exploit task-genre associations. This is outside the framework of this research, which concluded with the implementation and testing of the X-Site prototype. 


\section{Evaluation of Ta sk-Genre Filters}

\subsection{Overview}

In order to evaluate the effectiveness of the task-genre filters as implemented in the X-Site system, a user study was conducted to compare the performance of two versions of X-Site, a full version using task-genre filters and a baseline version with no filters. The experiment was conducted as a hybrid field/laboratory experiment, which provided some control over the variables while at the same time providing the necessary workplace context to conduct a contextual evaluation. The experiment used a within-subjects design, in which each participant used both versions of X-Site and completed four of eight experimental search tasks (see Table 7.1). The dependent measures were a range of search outcome metrics associated with system effectiveness, efficiency and user satisfaction. The queries and relevance assessments collected in the user study were then used to conduct a follow-up study to compare the mean average precision of results obtained from the two systems.

\subsection{Research Question and Hypotheses}

The intent behind the implementation of task-genre filters was to improve the precision and situational relevance of search results, in order to reduce the burden on the searcher to manually filter results, and to provide better support for work activities. One of the effects of filtering as 
implemented in X-Site is that documents that do not fall into any of the genre categories in the taxonomy are excluded from the results. Thus, across all searches, retrieval is limited to information-rich genres heavily used by the study population. The other effect is that genres not associated with the task profile for a specific search are removed from the results, ideally leaving behind a refined set of documents that are both relevant and useful.

An evaluation study was designed to test these effects and their impact on search outcomes, motivated by the following research question:

Research Question 6: Does the use of task-genre relationships to filter search results improve search outcomes?

It is expected that by increasing the precision and situational relevance of search results, the experimental system will allow searchers to find more relevant and useful documents, more quickly and with less effort. The following specific hypotheses relating to efficiency, effectiveness and user satisfaction were tested.

$\mathrm{H}_{1}$ The mean number of documents selected as relevant per search session will be higher in the Experimental condition than in the Baseline condition.

$\mathrm{H}_{2}$ Documents selected as relevant will have a lower mean rank and a higher mean level of usefulness per search session in the Experimental condition than in the Baseline condition.

$\mathrm{H}_{3}$ Participants in the Experimental condition will report higher levels of expected search task completion and higher levels of satisfaction than in the Baseline condition.

$\mathrm{H}_{4}$ The mean time to reach the first relevant document will be lower in the Experimental condition than in the Baseline.

$\mathrm{H}_{5}$ The mean time to complete a search session will be lower in the Experimental condition than in the Baseline. 
$\mathrm{H}_{6}$ The mean number of queries per search session will be lower in the Experimental condition than in the Baseline condition.

$\mathrm{H}_{7}$ The mean number of documents viewed from the hitlist per search session will be lower in the Experimental condition than in the Baseline condition.

$\mathrm{H}_{8}$ The mean rank of documents viewed from the hitlist per search session will be lower in the Experimental condition than in the Baseline condition.

\subsection{Method}

\subsubsection{Participants and Recruitment}

As in the previous studies, participants were recruited from within the study population of software services consultants. Recruiting was conducted in December 2005 through an email call for participation sent out to internal team mailing lists by one of the senior engineers in the software services group (Appendix 7). The sample was opportunistic, based on self-selection by the several hundred consultants who received the email announcement. There was no formal compensation offered for participation; however, thank-you letters were sent to all participants, and with their permission, circulated to their immediate managers.

Participants were thirty-two software services consultants working in a single product group in the company. They were geographically dispersed: nine were from Toronto, and the remainder worked in company offices in cities in ten U.S. states. Of the 32, three were women, which is proportionate to the gender distribution within the study population. Ages were distributed fairly evenly within the range of 26 to 55, and two participants were over 55. This was a highly educated group; all with university or college degrees, and more than a third with graduate degrees. Most participants reported educational backgrounds in engineering and computer science (26), but a small number (6) reported other educational backgrounds, including biology, chemistry and finance. Participants were well-experienced in this work; with an average of about eight years spent doing this type of software consulting. The group was well-balanced with 
respect to seniority: half were at corporate seniority levels 7-8 and half at seniority levels 9-10 (10 is the highest level). Based on their reported job titles, participants performed a range of roles as consultants, primarily as architects, information technology specialists, and developers.

\subsubsection{Variables}

\section{i. Independent variable}

The independent variable in this study was task-genre filtering. Two conditions were tested through use of two versions of the search system. The Experimental version of X-Site employed task-genre filters as described in the previous chapter. A Baseline version of X-Site was also built, using the same document collection, index and retrieval algorithm, but without any of the contextual components used to filter results.

\section{ii. Measures (Dependent Variables)}

Each search session was assessed using several effectiveness, efficiency and satisfaction measures, which are summarized in Table 7-1. The user's perception of how challenging it was to perform each search was collected in the post-task questionnaire, but was not used as a measure in the analysis due to feedback from participants that this question was ambiguous. Some interpreted it as an evaluation of the search process, and others of the intrinsic difficulty of the assigned search task.

\subsubsection{Experimental System}

Two versions of the X-Site search engine were used for the experiment. The Experimental version allows users to submit a task profile together with their query and filters results based on task-genre associations. The Baseline system accepts query input only and does not filter the results. The search results interface is the same for both versions. Both versions of X-Site used the same index and document collection, which consisted of about 10 gigabytes of data crawled from the corporate intranet, the corporate public web and extracted from two Lotus Notes 
databases. The collection was targeted to the user domain through the use of a customized seed

list of URLs provided to the crawler.

Table 7-1: Measures used to assess search sessions

\begin{tabular}{|c|c|c|}
\hline Measures & Definition & Instrument \\
\hline \multicolumn{3}{|l|}{ Effectiveness } \\
\hline Task Completion & $\begin{array}{l}\text { User's assessment of the likelihood that } \\
\text { search results will support task completion, } \\
\text { measured on a seven point Likert scale }\end{array}$ & Post-task questionnaire \\
\hline Relevant Documents & Number of documents identified as relevant & Usefulness log \\
\hline Rank of Relevant & $\begin{array}{l}\text { Mean rank of documents identified as } \\
\text { relevant }\end{array}$ & Search engine log \\
\hline Usefulness & $\begin{array}{l}\text { Mean usefulness rating of relevant } \\
\text { documents, measured on a scale of } 1 \text { to } 10 .\end{array}$ & Usefulness log \\
\hline \multicolumn{3}{|l|}{ Efficiency } \\
\hline Time on Task & $\begin{array}{l}\text { The time spent on the task, measured by } \\
\text { time elapsed from launch of browser until } \\
\text { participants clicks "I'm Done" button }\end{array}$ & $\begin{array}{l}\text { Experiment Wizard } \\
\text { database }\end{array}$ \\
\hline Time to First Relevant & $\begin{array}{l}\text { Time it takes to find first relevant } \\
\text { document, measured by time elapsed } \\
\text { between the first query until the first } \\
\text { relevant document is found. }\end{array}$ & $\begin{array}{l}\text { Experiment Wizard } \\
\text { database and Search engine } \\
\log \end{array}$ \\
\hline Document Viewed & $\begin{array}{l}\text { The total amount of effort expended } \\
\text { viewing documents, measured by the } \\
\text { number of documents opened. }\end{array}$ & Search engine log \\
\hline Rank of Viewed & $\begin{array}{l}\text { The amount of effort expended in reading } \\
\text { through the hitlist, measured by the mean } \\
\text { rank of documents viewed from the hitlist. }\end{array}$ & Search engine log \\
\hline Number of Queries & $\begin{array}{l}\text { The amount of effort spent in formulating } \\
\text { and reformulating queries, measured by the } \\
\text { number of queries submitted. }\end{array}$ & Search engine log \\
\hline \multicolumn{3}{|l|}{ User Satisfaction } \\
\hline Satisfaction & $\begin{array}{l}\text { The level of a user's satisfaction with the } \\
\text { search results, measure on a seven point } \\
\text { Likert scale. }\end{array}$ & Post-task questionnaire \\
\hline
\end{tabular}

\subsubsection{Search Tasks}

Eight search tasks were used in the experiment. These were developed following Borlund's (2003) guidelines for simulated work task situations in order to provide participants with enough context to interpret the information problem, to make relevance assessments from within the framework of their work experience, and to choose task profiles to accompany their queries. In order to design simulated situations that are relevant and meaningful, Borlund (2003) suggests that the target population be directly involved in their creation. However, due to the limited availability of the study population, online discussion groups used by the population were mined 
as a source of questions and problems. Twelve possible search tasks were developed based on the discussion group threads, of which eight were selected and revised in collaboration with one member of the study population. Two pilot sessions provided some additional feedback and lead to slight revisions of the search tasks prior to running the experiment.

\section{Table 7-2: Experimental Search Tasks}

Search Task Description

Search Task 1: You are installing WebSphere Portal Version 5.1 in a z/OS environment for the first time for a customer. The customer wants to know if there are any required pre-requisites for the operating system or WAS before installing Portal.

Search for reliable factual information on pre-requisites that you can provide to the customer.

\begin{tabular}{|c|c|}
\hline $\begin{array}{l}\text { earch Task 2: You are the technical lead on an engagement to deploy MQ Workflow in } \\
\text { pre-existing WAS environment. You need to figure out how to configure the customer's } \\
\text { pplications so that they can find the MQ Workflow JARS. Search for a document that } \\
\text { xplains how to configure WAS applications to use MQ Workflow. }\end{array}$ & $\begin{array}{l}\text { deployment } \\
\text { how-to }\end{array}$ \\
\hline $\begin{array}{l}\text { Search Task 3: You are mentoring a client's IT team in migrating from Dynamo } 5.0 \text { to } \\
\text { Websphere App Server. You're preparing a kick-off session for them to learn about the } \\
\text { process, best practices and pitfalls. } \\
\text { Search for information that you can use to prepare the presentation and/or give to the } \\
\text { client to support their learning process. }\end{array}$ & $\begin{array}{l}\text { migration } \\
\text { learn }\end{array}$ \\
\hline $\begin{array}{l}\text { earch Task 4: You have been called in to troubleshoot a problem resulting from an } \\
\text { grade to the current version of Websphere MQ on an AIX platform. Since the upgrade, } \\
\text { e customer's COBOL legacy application fails with return code } 2018 \text {. } \\
\text { earch for information that might be of use to you in identifying and resolving this } \\
\text { oblem. }\end{array}$ & $\begin{array}{l}\text { integration } \\
\text { solve a } \\
\text { problem }\end{array}$ \\
\hline $\begin{array}{l}\text { Search Task 5: You are advising a customer IT team on a long term project to develop a } \\
\text { J2EE } 1.4 \text { web services application for WAS. The customer asks for you advice on } \\
\text { whether to do session handling through stateful session beans or HTTP session objects. } \\
\text { Search for information that would be useful to them in making this decision }\end{array}$ & $\begin{array}{c}\text { design } \\
\text { decision }\end{array}$ \\
\hline $\begin{array}{l}\text { Search Task 6: You are working with a financial institution to architect an enterprise } \\
\text { service bus (ESB) solution in the framework of SOA. You are fairly new to ESBs and } \\
\text { you need to evaluate the different implementation patterns and decide on the best } \\
\text { approach for this client. Search for information that would help you identify the patterns, } \\
\text { evaluate them, and reach a decision. }\end{array}$ & $\begin{array}{l}\text { architecture } \\
\text { decision }\end{array}$ \\
\hline $\begin{array}{l}\text { Search Task 7: You are doing performance tuning for a Websphere Commerce } \\
\text { application. You know that there is an option that turns on logging for the JVM heap } \\
\text { garbage collection, but you can’t remember what it is. Search for information that } \\
\text { includes command syntax and how it is used. }\end{array}$ & $\begin{array}{l}\text { performance } \\
\text { tuning } \\
\text { factual }\end{array}$ \\
\hline $\begin{array}{l}\text { Search Task 8: You are trying to help a junior colleague who is on a remote } \\
\text { troubleshooting engagement for Websphere Portal. You suspect that slow authentication } \\
\text { response times are related to configuration issues with the LDAP server. Search for } \\
\text { information that would be of use to your colleague for problem determination and } \\
\text { resolution for this engagement. }\end{array}$ & $\begin{array}{l}\text { troubleshooting } \\
\text { solve a } \\
\text { problem }\end{array}$ \\
\hline
\end{tabular}

The eight search tasks used in the study are included in Table 7-2. The first part of each search task is a simulated work task situation to provide context, and the second part is an indicative request, which suggests what to look for (Borlund, 2003). It was not possible to cover all possible 
combinations of work tasks and information tasks in the taxonomies in the eight search tasks; however, the set chosen represents a range of more common combinations, based on review of numerous discussion lists and FAQs containing questions posed by software consultants. Also, attempts were made to balance the set according to relative complexity and level of detail, based on the data on work task characteristics gathered in the validation survey. Table 7-2 shows the intended task profile for each search.

\subsubsection{Study Design}

The order of assigned systems and search tasks was rotated to distribute the tasks evenly among participants and control for fatigue effects; each search task was performed by 16 participants, eight times with each version of the system (Table 7-3). The first 16 participants used the Experimental system (E) first, and the second 16 participants used the Baseline system (B) first.

Table 7-3: Experimental Design—System and Search Task Assignments

\begin{tabular}{|c|c|c|c|}
\hline Participant ID & Assignment & Participant ID & Assignment \\
\hline P1 & $\mathrm{E}(1,2) \mathrm{B}(3,4)$ & P17 & $\mathrm{B}(1,2) \mathrm{E}(3,4)$ \\
\hline $\mathrm{P} 2$ & $\mathrm{E}(2,3) \mathrm{B}(4,5)$ & $\mathrm{P} 18$ & $\mathrm{~B}(2,3) \mathrm{E}(4,5)$ \\
\hline P3 & $\mathrm{E}(3,4) \quad \mathrm{B}(5,6)$ & P19 & $\mathrm{B}(3,4) \mathrm{E}(5,6)$ \\
\hline $\mathrm{P} 4$ & $\mathrm{E}(4,5) \quad \mathrm{B}(6,7)$ & P20 & $\mathrm{B}(4,5) \mathrm{E}(6,7)$ \\
\hline P5 & $\mathrm{E}(5,6) \quad \mathrm{B}(7,8)$ & $\mathrm{P} 21$ & $\mathrm{~B}(5,6) \mathrm{E}(7,8)$ \\
\hline P6 & $\mathrm{E}(6,7) \quad \mathrm{B}(8,1)$ & $\mathrm{P} 22$ & $\mathrm{~B}(6,7) \mathrm{E}(8,1)$ \\
\hline P7 & $\mathrm{E}(7,8) \quad \mathrm{B}(1,2)$ & $\mathrm{P} 23$ & $\mathrm{~B}(7,8) \mathrm{E}(1,2)$ \\
\hline P8 & $\mathrm{E}(8,1) \quad \mathrm{B}(2,3)$ & P24 & $\mathrm{B}(8,1) \mathrm{E}(2,3)$ \\
\hline P9 & $\mathrm{E}(1,2) \quad \mathrm{B}(3,4)$ & $\mathrm{P} 25$ & $\mathrm{~B}(1,2) \mathrm{E}(3,4)$ \\
\hline P10 & $\mathrm{E}(2,3) \quad \mathrm{B}(4,5)$ & P26 & $\mathrm{B}(2,3) \mathrm{E}(4,5)$ \\
\hline P11 & $\mathrm{E}(3,4) \quad \mathrm{B}(5,6)$ & $\mathrm{P} 27$ & $\mathrm{~B}(3,4) \mathrm{E}(5,6)$ \\
\hline P12 & $\mathrm{E}(4,5) \quad \mathrm{B}(6,7)$ & $\mathrm{P} 28$ & $\mathrm{~B}(4,5) \mathrm{E}(6,7)$ \\
\hline $\mathrm{P} 13$ & $\mathrm{E}(5,6) \quad \mathrm{B}(7,8)$ & P29 & $\mathrm{B}(5,6) \mathrm{E}(7,8)$ \\
\hline $\mathrm{P} 14$ & $\mathrm{E}(6,7) \quad \mathrm{B}(8,9)$ & P30 & $\mathrm{B}(6,7) \mathrm{E}(8,1)$ \\
\hline $\mathrm{P} 15$ & $\mathrm{E}(7,8) \quad \mathrm{B}(1,2)$ & P31 & $\mathrm{B}(7,8) \mathrm{E}(1,2)$ \\
\hline P16 & $\mathrm{E}(8,1) \quad \mathrm{B}(2,3)$ & P32 & $\mathrm{B}(8,1) \mathrm{E}(2,3)$ \\
\hline
\end{tabular}

E: Experimental system, B: Baseline system Search tasks are represented by numbers (1-8) 


\subsubsection{Testing Environment and Instruments}

In order to control the flow of the experiment and collect data in electronic format, the Experiment Wizard, a custom-built Microsoft Access application, was used. The Experiment Wizard is similar in purpose and form to WiIRE, an application designed to run Experimental user studies over the Internet (Toms, Freund, \& Li, 2003); however, unlike WiIRE, it was not mounted on a server, but ran on a stand-alone laptop. The Experiment Wizard administered the assignment of search systems and search tasks, provided participants with study instruments, enabled participants to control their own progress through the study, and stored time-stamped user input directly into database tables.

Both the search systems and the Experiment and the Wizard were set up on the researcher's computer on the premises of the study site and connected to the corporate intranet. Participants were located remotely and completed the experiment by connecting their computers to the researcher's computer via Windows NetMeeting web conferencing software and using the remote screen control function. Sessions were conducted individually, and the researcher and participant were in contact by telephone throughout the session. Despite some minor technical difficulties, such as function and mouse keys that behaved differently than expected and slower than normal response times, running the study via NetMeeting resolved a number of technical and logistic issues:

- it allowed for remote participation, which was the only way to reach a large enough group of participants from the target population;

- $\quad$ participants did not need to download any software to take part, as NetMeeting is preinstalled on all Windows machines;

- no pre-session set-up was required, as most participants were already familiar with NetMeeting, and set-up was completed in the first five minutes of each one hour session; 
- it eliminated the need to develop a web application to run the experiment, which made the instrumentation of the study much simpler;

- it avoided the problem of variation in system settings and database permissions across participants, as they all conducted the study on the same computer.

The study instruments are described below; and screen-shots of the instrument as it appeared in the Experiment Wizard are presented in Appendix 8.

\section{i. Introduction and Consent forms}

The introduction and consent forms provided potential participants with a brief overview of the study and enough information about the study procedure and policies to allow them to provide informed consent. Consent was indicated by the participant providing their email address and clicking on the submit button. The email information was stored separately from the rest of the study data.

\section{ii. Background Information Questionnaire}

The background survey contained a number of questions used to establish a basic demographic profile of each participant (age, sex, education level) and a work profile (job title, years of experience, band (seniority) level). Participants were not required to answer any of these questions to proceed to the next step. In addition the "Job Band Level” question was explicitly marked as optional, as it is related to job performance and salary, and could be considered to be intrusive. Four participants chose not to answer this question.

\section{iii. Search Task Assignment Form}

The search task form provided the searcher with some basic instructions to follow for each search: to use the search engine provided, to inform the researcher when a useful document was found, and not to search for more than 15 minutes. The form also provided the text of the search task being assigned and included a link to the assigned search system. 


\section{iv. Post-Task Questionnaire}

The Search Feedback form contained a series of questions on the user's experience searching on a given search task. Questions were designed to assess their level of familiarity with a topic, their satisfaction with the search experience, the degree to which the search results would support task completion, and the difficulty of conducting the search. Questions were assessed using seven point Likert scales. Scales were reversed for some of the questions (1=high, 7=low) in order to force participants to think about their response to each question. Two open-ended questions asked participants to provide feedback on what kind of information they would have liked to have found, and where they would normally go to find this type of information, if they were not satisfied with the search results.

\section{v. Post-Study Questionnaire}

The final feedback form contained open-ended questions on the search engine in general and the two different interfaces used in the study. This allowed participants to suggest features and provide general feedback on the system.

\section{vi. Usefulness Log}

A structured log form was used by the researcher to record verbal responses from participants when they found documents that they identified as useful. Participants were asked to indicate when they found a useful document, rate it on a scale of one to ten for usefulness, and explain why they thought it would be useful. A ten point usefulness scale was used because it is a familiar and intuitive scale, which is often used in informal verbal contexts. Participants had very little trouble assigning these scores, and many participants assigned even finer-grained scores, such as 8.5. Participants' verbal responses to these questions were recorded on a log sheet (Appendix 9). 


\subsubsection{Procedure}

On average, study sessions took 50 minutes (range 25-70 minutes). All sessions were conducted individually and remotely via Windows NetMeeting and telephone. The experimental procedure was as follows:

- At a pre-arranged time, a participant was contacted by telephone and verbally guided through the process of establishing a NetMeeting connection and gaining remote control over the study computer.

- The participant opened the Experiment Wizard, read the Introduction and Consent Forms and submitted an email address as indication of informed consent.

- The participant completed the Background Information Questionnaire.

- The participant opened the Search Task Assignment Form, was assigned the first search task and was provided with a link that opened up a browser with one of the versions of XSite. Participants were able to move between the two windows to review the assigned search task at any time during the search.

- The participant began to search and informed the researcher verbally when he or she found a useful document. For each useful document, the participant was asked to rate the document for usefulness on a verbal scale of one to ten, and explain why he or she thought it would be useful. The participant's feedback was recorded on the Usefulness Log. When the participant felt he or she had enough information, or felt that under normal searching circumstances he or she would stop searching, or had been searching for 15 minutes, he or she returned to the Search Task Assignment Form and clicked the "I’m Done” button. Participants were not required to stop after 15 minutes, but were simply reminded at this point that they could move on. 
- The participant completed the Post-Task Questionnaire.

- The participant returned to the Search Task Assignment Form and was assigned the second search task with the same version of X-Site, and repeated the searching steps. For the second and third assigned search tasks, the participant was assigned the other version of X-Site.

- Upon completion of the fourth search task, the searcher completed a brief Post-Study Questionnaire asking for general feedback on the search system.

- Participants were thanked for taking part in the experiment and were given the opportunity to ask any questions they had about the search system or study.

\subsubsection{Data Collection and Analysis}

Quantitative data was prepared for analysis by aggregating data stored in various instruments and summarizing it at the level of each of the $128(32 \times 4)$ search tasks. For measures that were recorded at a higher granularity (e.g. documents retrieved during a search task), the mean value per search task was used. Data was gathered into an Excel spreadsheet, and imported into SPSS 15.0 for analysis. Qualitative data was stored and analyzed separately.

Hypothesis testing was conducted by comparing the group means of the dependent variables under the system condition (Baseline vs. Experimental). As this experiment has one condition, two dependent groups and non-parametric measures, the Wilcoxon signed ranks test was used ${ }^{7}$.

Content analysis of the qualitative data collected focused on two questions: how participants assessed the usefulness of documents, and their impressions of searching with X-Site.

\footnotetext{
${ }^{7}$ The same significant differences were identified with both parametric and non-parametric tests for these measures. Non-parametric test results are reported.
} 
Although 32 participants took part and each completed four search sessions, in some cases participants did not respond to all questions, in one case the session timing was lost, and in about one quarter of search sessions no relevant documents were found. This high number of failed searches resulted from a range of factors, including the fact that participants were asked to “search as they normally would.” In many cases they quickly abandoned searches, indicating that at that point they would normally give up on the search system and ask a colleague or figure out the problem on their own.

\subsection{Results}

\subsubsection{Data Preparation}

Quantitative data were examined for normality. All measures failed the normality tests except for Time on Task. Histograms (Appendix 10) indicate that some measures are skewed, some have an exponential distribution, and others are bi-modal. Although transformation may have brought some of the measures into the normal range, it is unlikely that the bi-modal measures would have responded, therefore parametric tests were conducted. Outliers were identified using boxplots, and the analysis was conducted with extreme outliers removed. However, as the results did not change, results are reported including outliers, which are reflections of the natural variation in human searching behaviour rather than errors in data collection. Only instances of significant differences at the .05 significance level or less are discussed.

\subsubsection{Search Behaviour Summary}

Participants' search behaviour in the experiment was quite similar to what is known of search engine use in general. Overall, using the two systems, participants were successful in finding at least one relevant document in $78 \%$ of searches, and found 1.7 (range 0 to 5) relevant documents on average per search task. On average, participants spent just over eight minutes, viewed 3.1 
documents from the hitlist, and issued 2.7 queries per search task. Participants tended to use

longer queries (mean 4.1 terms) and more advanced search syntax than the typical web searcher.

\subsubsection{Hypothesis testing}

A Wilcoxon signed ranks test was conducted to compare the means of all measures assessed at the session level for the two systems and paired by participant.

Table 7-4: Results of Wilcoxon Signed Rank test for all dependent measures

\begin{tabular}{|c|c|c|c|c|c|c|}
\hline & & $\mathbf{N}$ & $\begin{array}{l}\text { Mean } \\
\text { Value }\end{array}$ & Std. dev. & Z statistic & Sig \\
\hline \multicolumn{7}{|l|}{ Effectiveness } \\
\hline \multirow[t]{2}{*}{ Task Completion } & Baseline & 64 & $2.92 *$ & 2.03 & \multirow{2}{*}{-.831} & \multirow{2}{*}{$n s$} \\
\hline & Experimental & 63 & $3.21 *$ & 2.29 & & \\
\hline \multirow{2}{*}{$\begin{array}{l}\text { Number of } \\
\text { Relevant } \\
\text { Documents ** }\end{array}$} & Baseline & 64 & 1.73 & 1.24 & \multirow[b]{2}{*}{-.358} & \multirow[b]{2}{*}{$n s$} \\
\hline & Experimental & 64 & 1.66 & 1.43 & & \\
\hline \multirow{2}{*}{$\begin{array}{l}\text { Rank of Relevant } \\
\text { Documents } * * *\end{array}$} & Baseline & 53 & 3.73 & 3.06 & \multirow{2}{*}{-.443} & \multirow{2}{*}{$n s$} \\
\hline & Experimental & 47 & 4.17 & 4.20 & & \\
\hline \multirow{2}{*}{$\begin{array}{l}\text { Usefulness of } \\
\text { Relevant } \\
\text { Documents**** }\end{array}$} & Baseline & 47 & 7.84 & 1.66 & \multirow[b]{2}{*}{-1.111} & \multirow[b]{2}{*}{$n s$} \\
\hline & Experimental & 41 & 7.56 & 1.89 & & \\
\hline \multicolumn{7}{|l|}{ Efficiency } \\
\hline \multirow[t]{2}{*}{ Time on Task } & Baseline & 63 & $7: 25$ & 3:03 & \multirow{2}{*}{-2.30} & \multirow{2}{*}{$\mathrm{p}<.05$} \\
\hline & Experimental & 63 & 8:58 & $4: 15$ & & \\
\hline \multirow{2}{*}{$\begin{array}{l}\text { Time to First } \\
\text { Relevant*** }\end{array}$} & Baseline & 53 & $1: 42$ & $1: 54$ & \multirow{2}{*}{-1.61} & \multirow{2}{*}{$n s$} \\
\hline & Experimental & 45 & $2: 44$ & 3:07 & & \\
\hline \multirow{2}{*}{$\begin{array}{l}\text { Number of } \\
\text { Documents } \\
\text { Viewed** }\end{array}$} & Baseline & 64 & 3.14 & 1.72 & \multirow[b]{2}{*}{-.749} & \multirow[b]{2}{*}{$n s$} \\
\hline & Experimental & 64 & 3.09 & 2.17 & & \\
\hline \multirow{2}{*}{$\begin{array}{l}\text { Rank of } \\
\text { Documents } \\
\text { Viewed } * * * * *\end{array}$} & Baseline & 62 & 4.09 & 3.32 & \multirow[b]{2}{*}{-.155} & \multirow[b]{2}{*}{$n s$} \\
\hline & Experimental & 59 & 3.97 & 3.00 & & \\
\hline \multirow{2}{*}{$\begin{array}{l}\text { Number of } \\
\text { Queries }\end{array}$} & Baseline & 64 & 2.56 & 2.05 & \multirow{2}{*}{-.822} & \multirow{2}{*}{$n s$} \\
\hline & Experimental & 64 & 2.73 & 1.92 & & \\
\hline \multicolumn{7}{|l|}{ Satisfaction } \\
\hline \multirow[t]{2}{*}{ Satisfaction } & Baseline & 64 & $3.00^{*}$ & 2.05 & \multirow{2}{*}{-1.321} & \multirow{2}{*}{$n s$} \\
\hline & Experimental & 64 & $3.42 *$ & 2.09 & & \\
\hline
\end{tabular}

* measurement scales are inverted (1=high; 7=low).

** includes all sessions (zero or more documents)

*** includes sessions with one or more relevant documents.

$* * * *$ includes sessions with one or more assessed relevant documents.

$* * * * *$ includes sessions with one or more viewed documents 
The Wilcoxon signed ranks test is suitable for use with non-parametric data of an ordinal or continuous type taken from two dependent samples. It is the non-parametric equivalent of the paired t-test. Data on all measures is reported in Table 7-4.

Results do not support the hypotheses. In fact, based on the metrics there is surprisingly little difference in outcomes between the two systems. The only statistically significant difference is in Time on Task, which indicates that it took longer to complete a search task using the Experimental system. For most of the measures, the standard deviation was higher for the Experimental system, suggesting that there was more variability in the values. A more detailed analysis of some of the measures is reported below.

\section{i. Number of Relevant Documents and Task Completion}

Participants identified a total of 215 relevant documents over all 128 search sessions, ranging from zero to five relevant documents per session. The frequency distribution of this measure for the two systems is shown in Figure 7-1.

Figure 7-1: Number of relevant documents by search task and system

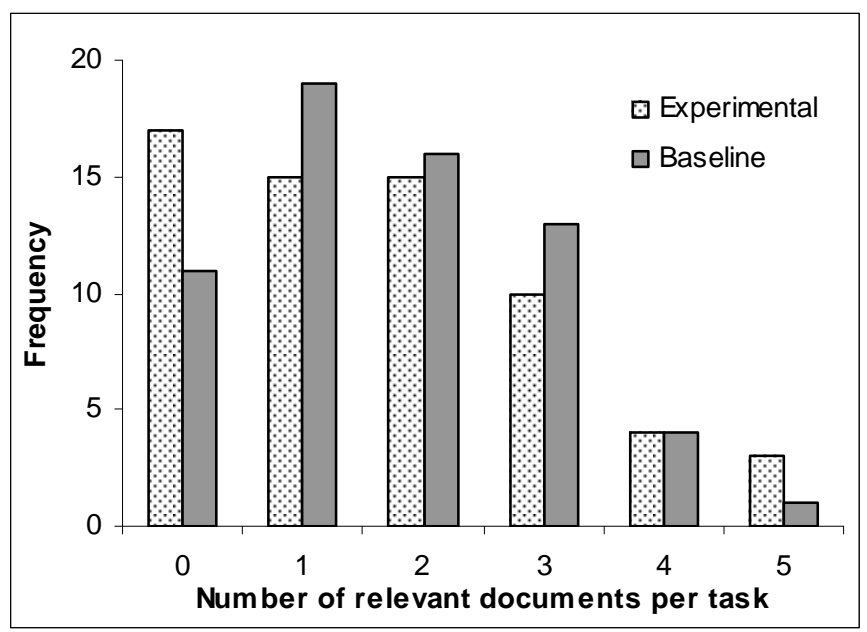

Results for the Baseline system show that most sessions resulted in one to three relevant documents; whereas results for the Experimental system shows relatively more searches which 
resulted in no relevant documents (zero) and many (five). A similar pattern appears in the distribution of Task Completion scores (Figure 7-2), in which results are higher on the two extremes of the scale (1 and 7) for the Experimental system.

Figure 7-2: Distribution of task completion scores by search task and system

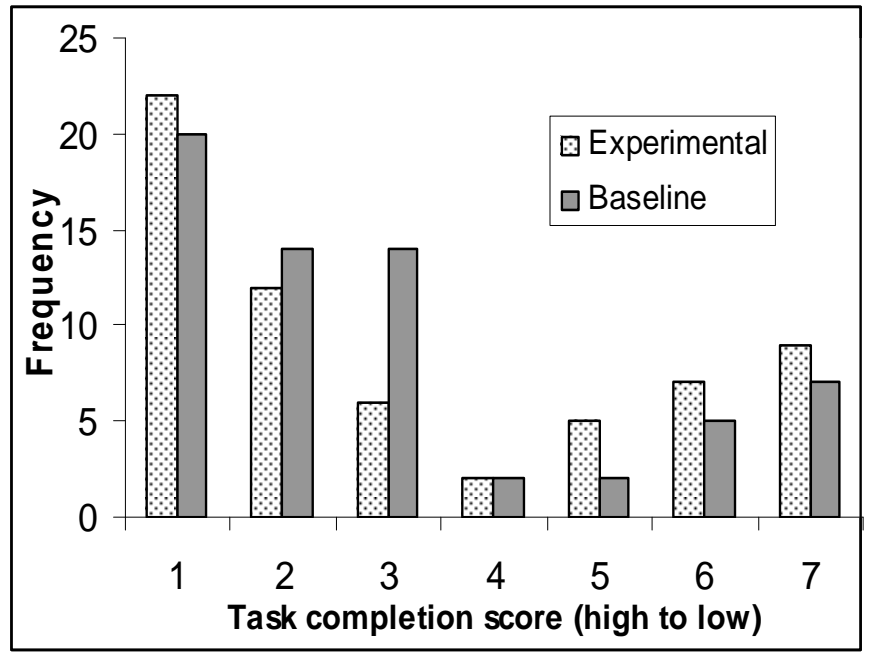

\section{ii. Rank and Usefulness of Relevant Documents}

The mean hitlist rank and usefulness scores of relevant documents were not significantly different for the two systems. However, the distribution of mean hitlist rank per session (Figure 7-3) shows a similar trend of a flatter and broader pattern for the Experimental system relative to the Baseline, with fewer documents selected from the first half of the hitlist page, and more from the second half of the page and beyond. The distribution of usefulness scores (Figure 7-4) indicates that fewer documents found with the Experimental system received the highest score (10); while more documents were given mid-range scores (3 to 6).

In summary, the performance of the Experimental system was less consistent than that of the Baseline system, as evidenced by the flatter distributions and higher standard deviation values for most of the measures. 
Figure 7-3: Distribution of mean rank of relevant documents per search task by system

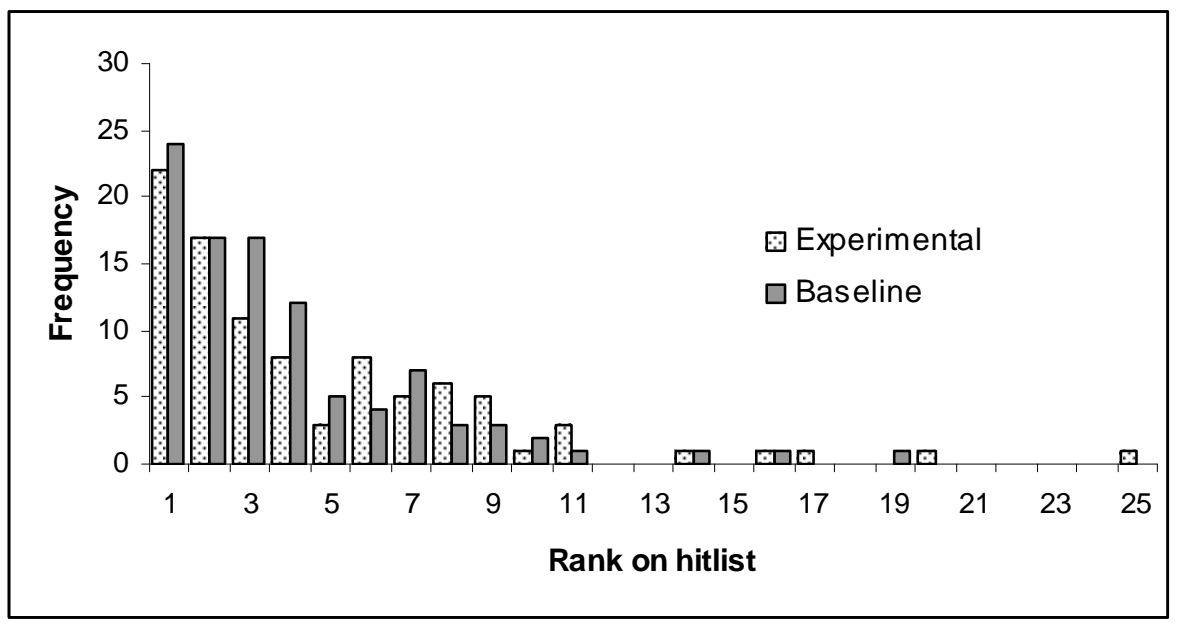

Figure 7-4: Distribution of usefulness scores of relevant documents by search task

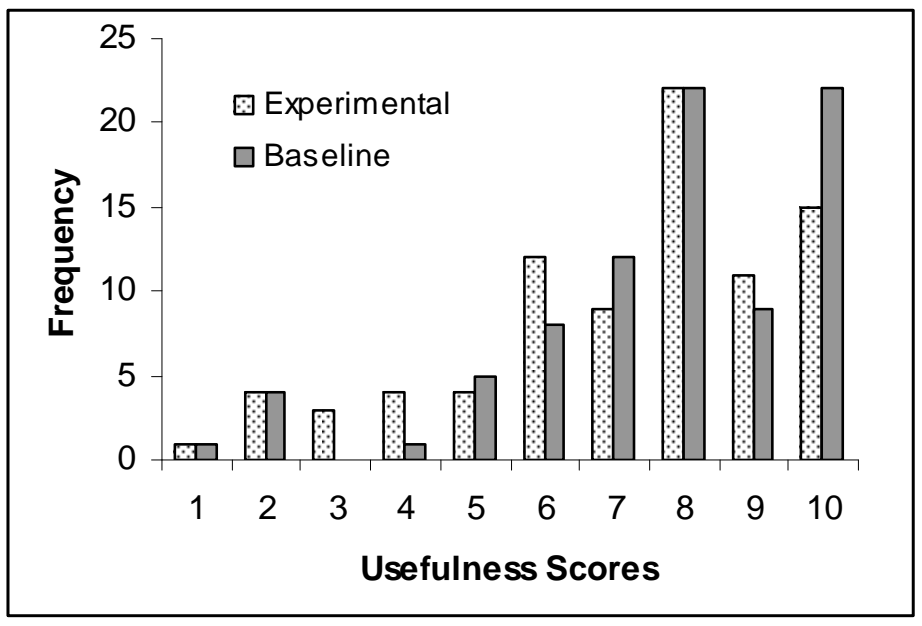

\section{iii. Variation by Search Task}

One possible explanation for this lack of consistency is variation in system performance across the search tasks. To assess the possible effect of search task, Mann Whitney tests were performed using a subset of measures from the two systems related to effectiveness and user effort for each of the eight search tasks. The measures tested were: Task Completion, Satisfaction, Usefulness, Rank of Relevant and Number of Queries. Significant differences between the two systems were found for only two of the eight search tasks. For Search task 2, there was a significant difference in the mean number of queries (Baseline $=4.2$, Experimental $=2.14, \mathrm{U}=4.50, \mathrm{p}<.05$ ). For search 
task 8, there were significant differences in the means for Task Completion (Baseline $=1.86$, Experimental=3.00, $\mathrm{U}=6.00, \mathrm{p}<.05$ ) and Satisfaction (Baseline $=2.00$, Experimental $=3.33, \mathrm{U}=$ 10.00, $\mathrm{p}<.05$ ). For Search task 2, the Experimental systems seems to have performed more effectively, as users needed significantly fewer queries to achieve similar levels of task completion and satisfaction, while for search task 8, the Experimental system performed somewhat less effectively, as Satisfaction and Task Completion scores were significantly lower than for the Baseline system (scale 1 to 7, 1=high, 7=low).

A closer look at these two search tasks provides some indication of the source of variation in the performance of the Experimental system. Search task 2 has an intended task profile of work task $=$ deployment and information task $=$ how-to. All participants using the Experimental system selected the "how-to" option and chose deployment, implementation or installation. Of the documents selected as relevant for search task 2 in both conditions, 65\% were tagged with genre classes that would be retrieved by the filter associated with the intended task profile for search task 2. In the Experimental condition, multiple participants selected the same small set of relevant documents, which were found less often in the Baseline condition, where there was less overlap in the pool of relevant documents. So, for this search task, users of the Experimental system were able to reach relevant documents with less effort (fewer queries) than with the Baseline system, and participants were able to consistently find a small number of highly relevant documents.

The intended task profile of search task 8 consists of work task = troubleshooting and information task $=$ problem solving. However, in this search task, participants in the Experimental condition selected a wide variety of work task options to describe this search task, and frequently changed their selection in the course of searching. Of the documents selected as relevant for search task eight in both conditions, only 50\% were tagged with genre classes associated with the intended task profile, according to the TGAM. This was the lowest level of accuracy of the TGAM among 
all search tasks in the experiment. For this search task, participants were less satisfied with the Experimental system, which seems to have filtered a number of highly relevant documents out of the hitlist due to inaccuracies in the TGAM and/or the task profiles selected by participants.

\subsubsection{User Perceptions and Feedback}

\section{i. Usefulness Criteria}

When participants found a relevant document, they were asked to assign a usefulness score on a scale of 1 to 10 , with 1 being the least useful, and to explain why they thought it would be useful. Participants mentioned a wide range of criteria for assessing usefulness, including generic criteria related to information quality and task-dependent criteria.

The most commonly mentioned a criterion was that the document was related to the topic in some way. Useful documents that received low scores often contained only background information to the topic, or a "clue”(P19) or "pointer”(P4,7) that would "lead me to what I need” (P32). Highly useful information was described as being "right on topic”(P18,19,30) and specific to the scenario (P10). Consultants looked for information at a "decent level”(P21), with the amount of detail required for the topic and the audience, which in some cases was the customer. Lower scoring documents were frequently described as being too "high level”(P5, 24, 28) or "very general”(P16).

The issue of appropriateness for the task was expressed by participants in terms of document genre and purpose. For example, on-topic marketing information was dismissed by participants as “junk”(P19) or “marketing stuff—I wouldn’t use it”(P27). One participant chose one document because the description read "best practices for real life scenarios"(P10) and another because it "suits the task of putting together a presentation”(P10). Ready-to-use, “canned materials”(P27) were preferred, as they could be given directly to the customer. 
Authority and currency criteria were frequently mentioned by participants. Currency was assessed based on the version of product or technology discussed, and the date of publication. Participants were often looking for “very current material”(P4) written within the past few months, but would also select less current materials, hoping to extract some useful information. Authority was assessed based on acquaintance or reputation of the author and the organizational source of the information. Participants often selected documents because they personally knew the author or the author was from their team, and therefore had actual experience in the field and could be trusted. Comments such as “It’s from W---- -- it must be good”(P27) were common.

Some document features that participants described as adding value included: structure, references and novelty. They preferred documents that they could easily extract needed information from, and thus preferred shorter, less dense documents with tables of contents, abstracts and clearly defined sections. Documents which “may have something”(P9), but required time for detailed reading were often assigned low usefulness scores. Participants often preferred documents which were "part of a series”(P3), had references, links or names of experts, which would allow them to find more information if needed. One participant noted that "it gives me names, so it’s wonderful”(P31). Finally, a small number of participants noted that novel information, “something I would not normally have found”(P19) was particularly useful. Participants appreciated documents from which they could learn something, for example: “[it’s] good to know there is a team doing this”(P1).

To summarize, marginally useful documents were typically related to the topic in some way, but were flawed with respect to level of detail, specificity, currency or task-appropriateness, or could not be assessed because the contents were difficult to make sense of quickly. The most useful documents were typically both on topic and in a form well-suited to the task, as well as being current, authoritative and easy to understand and use. Participants appreciated information that 
was novel and informative to them personally, and which provided pointers to related sources of information.

\section{ii. System Features and Design}

Participants completed a general feedback form after completing their four search tasks, which asked them to provide comments on the design of the two interfaces and to indicate things they did or did not like about the two systems. The range of general comments reflected the variation in performance of the Experimental system. As one participant noted, the Experimental system was "great when it worked and not so great when it didn't” (P 26). Some participants noted a positive impact of the filtering in the Experimental system: "I felt like I was able to get more information, less 'stuff'” (P 28). Others, despite a stated predisposition to like the Experimental system, found the opposite: "I prefer the experimental interface, although the simpler one seemed to return better results” (P 10).

Some common threads were evident in participants’ comments. Several participants indicated their willingness to put more effort into refining their searches in order to improve the outcome. One participant noted that he was "quite happy to tune the search" (P 6), and another noted that “it isn’t like those two extra pull-downs slowed me down much, so if it narrowed down the hits it was good” (P13). However, the difference in performance needs to be noticeable to justify the effort: "Frankly, if I know that adding something really will help I will do it, but in this case I preferred the basic interface” (P8). Other participants were less interested in using a more complex search interface, and gave feedback such as, "multiple search fields slows down the process" (P30) and expressed more bluntly, "get rid of the taxonomy” (P5).

Two main criticisms of the Experimental interface were the lack of transparency and user control over what the task profile selections were doing: "I want to know what I have searched” (P5) and "I’m leery of filters because sometimes they limit the search too much" (P26). One 
participant noted that he avoided selecting task profiles because he did not understand the effect. The second critique was that in some cases, selection of task profiles was too difficult. Some participants found that multiple task profiles could be inferred from a single search task, and suggested that the system should allow for multiple selections. With respect to information tasks, the main difficulty seems to have been in understanding the distinctions between the five options. One participant referred to the profile options as "brain teaser categories—so similar they intersect” (P17).

Participants also suggested specific features that would improve the system. The lack of support for Boolean syntax was raised repeatedly by participants, who claimed to make heavy use of this type of syntax in their normal interaction with search engines. In fact, many participants used advanced query syntax of one form or another during the experiment, assuming that it was supported by X-Site. A number of additional system enhancements were suggested:

- identification of the source of documents on the hitlist (intranet, public web, specific websites);

- a means of limiting the search results by document source, genre and date;

- identification of authors names on the hitlist;

- removal of duplicates in hitlist.

\subsubsection{Analysis of Contextual Components}

The X-Site implementation of task-genre relationships is dependent upon a number of contextual components: the genre classifier, the Task Genre Association Matrix (TGAM) and the task profiles selected by the searchers. Together, these components determine which documents will be filtered out for each search. Separate analyses were conducted to assess the performance and accuracy of each of these components and to provide further insight into the results of the experiment. 


\section{i. Task Profiles}

A comparison of the task profiles selected by study participants against the intended task profiles for each search task found that approximately $60 \%$ of participants' work task selections and $72 \%$ of their information task selections matched the intended values. Some variation in task profile selection is a natural reflection of participants' different perceptions and experience, but may also reflect lack of clarity in search task descriptions or ambiguity inherent in the task taxonomies. While it is difficult to assess the impact of this variation on study results, of the 17 sessions which retrieved no documents in the Experimental condition, 12 used task profiles that were different from the intended profiles. Also, as noted above, search task 8, which had the highest level of task profile variation, showed the worst performance of the Experimental system relative to the Baseline.

\section{ii. Genre Classifier and TGAM}

To assess the accuracy of the X-Site genre classifier in the experiment, documents selected by participants as relevant were manually classified based on the genre taxonomy, and the systemassigned classifications were compared to the manual. Of the relevant documents retrieved by the Baseline system and automatically classified, 58\% were assigned at least one of the categories assigned manually or were correctly left unclassified, 13\% were classified differently, and 29\% were incorrectly left unclassified. The last category of relevant documents would not have been available to searchers in the Experimental conditions regardless of the task profile selected, since all unclassified documents are removed by the genre filter.

Based on the manual genre classifications, $73 \%$ of all relevant documents were in accordance with the TGAM for the intended task profiles of search tasks for which they were retrieved. The remaining $27 \%$ includes documents that are topically but not situationally relevant, document genres that are not included in the taxonomy, and documents that the TGAM did not identify as associated with the task profile. However, based on the automatic genre classifications performed 
by X-Site in the Baseline condition, only $48 \%$ of relevant documents were in accordance with the TGAM. This suggests that the number of relevant documents filtered out of search results in the Experimental condition, through a combination of classification error and inaccuracies in the TGAM, may have been as high as $50 \%$.

\subsubsection{Discussion}

Three types of results have been reported: study metrics, user perceptions and feedback, and analyses of the performance of the contextual components. Across all tasks, the systems differed only on the basis of the time spent searching. This difference can be explained in part by the relative novelty and complexity of the Experimental search system. The lack of difference on other measures seems to be the result of inconsistent performance by the Experimental system, as compared to the Baseline, as most of the measures have a broader distribution. Furthermore, analysis of the two search tasks that showed some differences between the systems suggests that when all the components of the Experimental system were functioning well, the task-genre filters did have a positive effect and the system worked well; however, when there was a high level of error, performance suffered because good results were removed from the results list. This suggests that further work needs to be done to improve the performance of these components before reaching any clear conclusions about the contribution of task-genre relationships to workplace search. Findings from the study will be useful to guide those improvements.

Participants' feedback on what makes a document useful certainly adds weight to the claim that situational relevance is important, as they definitely preferred documents that could be applied directly to the problem at hand. A number of participants also indicated that they would be willing to put more effort into searching if it would help them obtain better results, which supports the approach of having searchers supply the task profiles. However, searchers using the Experimental system were somewhat frustrated by not understanding how the system worked and what the filters were doing, which may have hindered their effective use of the filters. On the 
other hand, it was surprising to see how quickly and simply some of the participants searched, and how in many cases they were satisfied with a single document that only contained a starting point or clue. This may be evidence of satisficing behaviour, or may be a learned behaviour based on the low return on investment with workplace search systems in the past. In either case, the implications of this behaviour for this study were that searchers were not very discriminating of system performance. For this reason, a more controlled follow-up study was designed to compare retrieval performance between the two systems.

\subsection{Retrieval Precision Experiment}

Data collected in the user study was re-purposed to create a small test collection used to conduct a small-scale batch retrieval experiment.

\subsubsection{Method}

In order to evaluate retrieval precision, a test collection was created consisting of a set of search tasks, and for each task: a set of queries and associated task profile and a pool of documents judged to be relevant for that task. Although the test collection constructed here is on a very small scale, it is unique in that the queries and assessments were made by the study population in a naturalistic search setting.

\section{i. Search tasks and queries}

The eight search tasks developed for the user study were used in this follow-up experiment. For each search task, a set of 10 queries were selected from the pool of queries submitted by participants in the user-study. There was a total of 343 (326 unique) queries submitted in the user-study, ranging from 24 to 50 per task. The 10 queries selected for each task were error-free and were chosen to represent a range of lengths (one to nine terms). Rather than using the task profiles selected by participants in the user study, which varied considerably for most of thesearch 
tasks, queries submitted to the Experimental system were accompanied by the intended task profiles used to design the search tasks.

\section{ii. Relevance Assessments}

All documents identified as relevant by participants in the user study were considered to be relevant for a given search task, and all other documents were considered to be non-relevant. The number of documents judged as relevant was quite low, ranging from seven (Task four) to 31 (Task six). A binary relevance measure was used, as the amount of data available was too limited to make use of graded relevance assessments.

\section{iii. Search System and Collection}

The same Baseline and Experimental systems used in the full study were used; however, the document collection was not identical. Since a number of months separated the two studies, a new crawl of the same information space was conducted and used as the document collection for the follow-up study. As a result, some of the documents judged as relevant were no longer available in the document collection.

\section{iv. Procedure}

For each task, the 10 queries were submitted to the two versions of the system. Queries were submitted as-is to the Baseline system and together with the intended task profiles to the Experimental system. The first 20 results for each query were collected and relevant documents were identified by comparing the URLs of retrieved documents to the URLs of documents identified as relevant. For each query, retrieval precision (number of relevant documents / total retrieved) was calculated at four points in the hitlist: at 5, 10, 15 and 20 results. Mean Average Precision (MAP) was calculated for each search task and system by averaging the precision across the 10 queries at each of the four points. 


\subsubsection{Results}

The MAP values obtained are generally very low, as a result of the small number of relevant documents in the pool for each search task and the change in the underlying document collection. The three search tasks (one, four, and eight) that had extremely low precision values (under .05 at all levels) were excluded from the analysis, as only one or two relevant documents were retrieved over all the queries.

Figure 7-5: MAP over five search tasks for the two systems

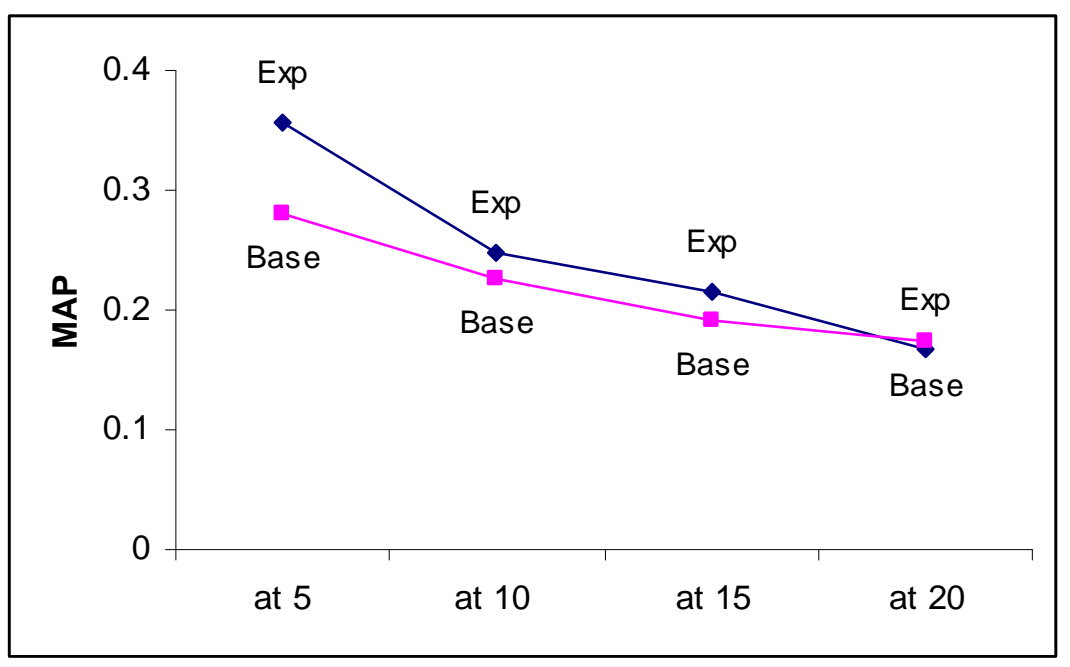

Figure 7-5 shows the average precision across the five search tasks included in the analysis, indicating a small difference in the MAP at 5 for the two systems, which tapers off and equalizes by 20. Graphs of MAP by search task (Figures 7-5 to 7-9) show that this trend of the Experimental system obtaining higher precision values at the top of the hitlist is quite pronounced in the results for several of the search tasks. Only search task six shows lower MAP values for the Experimental system at all levels. Significance tests were not performed due to the small number of queries, relevance assessments and search tasks, thus conclusive differences in performance between the two systems cannot be claimed. However, the trend suggests that there is some beneficial effect of the genre filter removing non-relevant results, thereby concentrating the relevant results in the set at the top of the hitlist. The effect is small; however, as noted above, 
performance issues with the current implementation of the X-Site system need to be remedied in order to perform a more robust evaluation of the task-genre effect.

\subsection{Summary}

This chapter presented an evaluation of task-genre relationships as implemented in the X-Site System. X-Site represents a unique and experimental implementation of this type of dynamic, contextual filtering of search results based on task-genre relationships. Two studies were conducted to assess X-Site: an experimental user study and a follow-up study of retrieval precision. Results of the user-study are inconclusive, due to the lack of significant differences, but both studies offer some indications that task-genre relationships have potential that is not realized in the current prototype. Feedback from participants on their search results reinforced the assumptions that informed the design of X-Site - that documents which are suited to the task at hand are preferred over documents which are merely topically relevant. Results point to a number of areas for improvement, including:

- the accuracy of the genre classifier;

- the flexibility for selecting task profiles should be increased by allowing multiple selections;

- system transparency, so searchers understand how the filters work;

- a ranking vs.filtering approach should be considered, to reduce the effect of filtering error. 
Figure 7-6: Task 2: Comparison of MAP for 2 systems

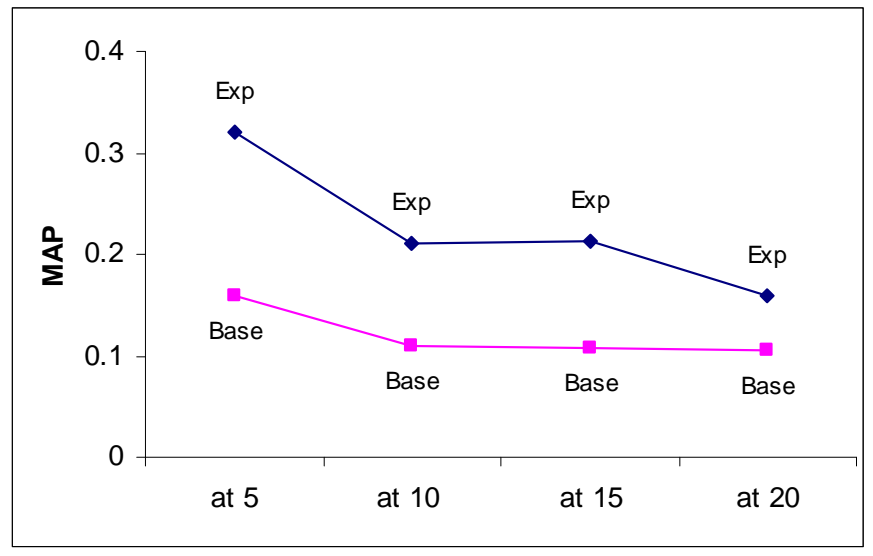

Figure 7-8: Task 5: Comparison of MAP for 2 systems

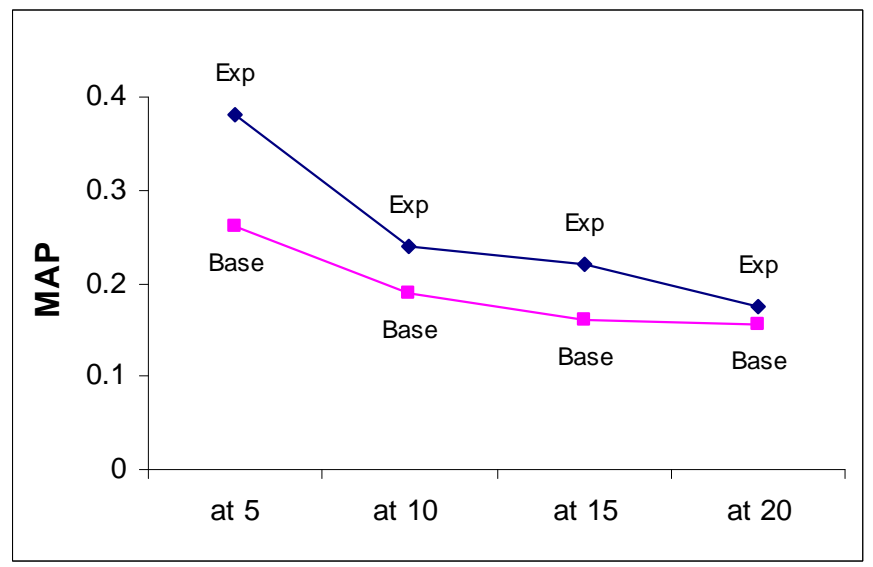

Figure 7-7: Task 3: Comparison of MAP for 2 systems

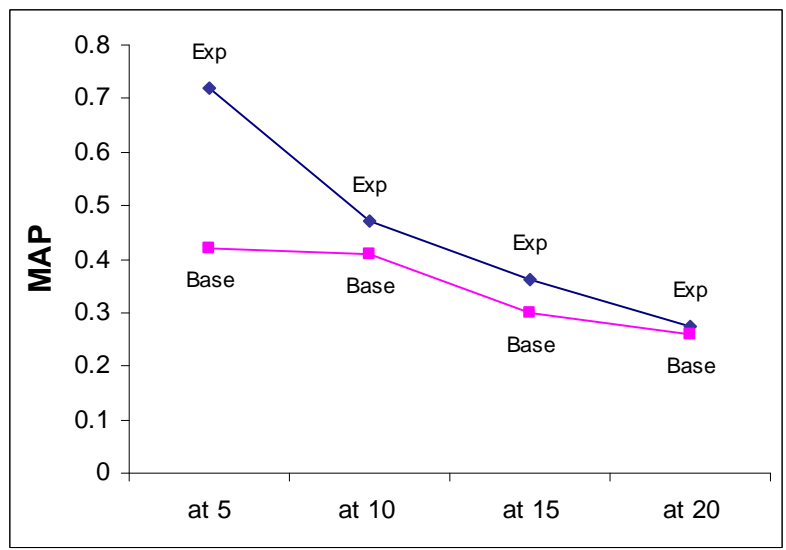

Figure 7-9: Task 6: Comparison of MAP for 2 systems

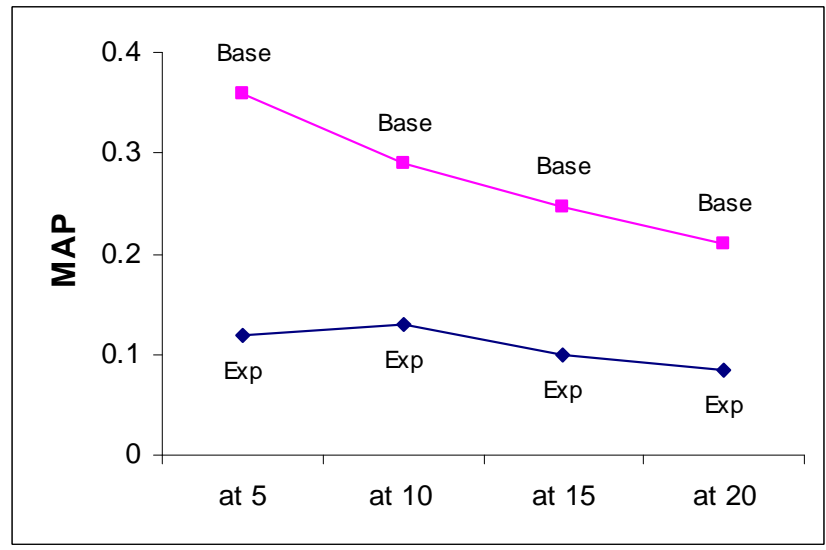

Figure 7-10: Task 7: Comparison of MAP for 2 systems

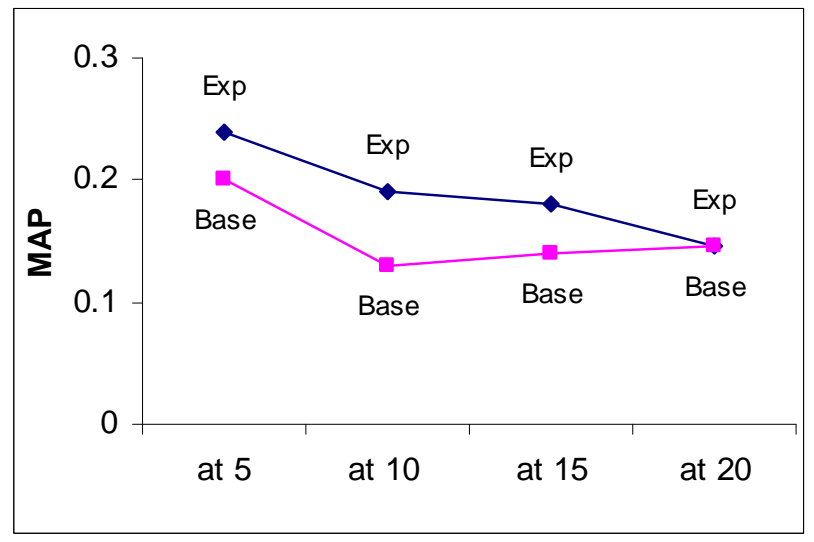




\section{Summary and Disc ussion}

\subsection{Overview}

This research has explored and evaluated an innovative contextual approach to workplace IR. Results confirm the highly situated nature of information behaviour in this domain and provide support for the idea that contextual factors establish selection criteria for information. Focusing on task-genre relationships, this work shows that such relationships can be modeled and implemented in an IR system. Evaluation of the relationship between task and genre implemented as a filtering component did not show significant differences in retrieval performance. However, the evaluation indicated the potential contribution of task-genre relationships in IR systems, provided that some implementation issues can be remedied. This chapter will provide a summary of the findings over the three phases of the study and a discussion of the implications organized by the main themes in the research.

\subsection{Summary of Findings}

This section will provide a summary of research findings for the three phases of the study.

\subsubsection{Phase 1 Findings}

RQ1: Which contextual factors are important in determining how software engineers search for information and select documents? 
A set of ten key factors were identified in the course of the work domain study. These fall into four contextual spheres, as follows:

- Person: work role, professional expertise, familiarity with subject

- Project: length, stage of completion, client importance/knowledge, system maturity

- Work tasks: type of software engineering task, type of consulting task

- Problem: type of information task

RQ2: What relationships exist between contextual factors of the searcher at work and information searching and selecting behaviour?

The relationships between these factors and aspects of information behaviour were summarized in a Model of Contextual Effects (Figure 4.1), which consists of four main components: contextual factors, information access constraints, information utility criteria, and information seeking strategies. The contextual factors have a direct effect on information access constraints and selection criteria, as well as indirect effects on information seeking strategies, as the latter are shaped by the necessity of coping with access constraints and the desire to find information that meets the selection criteria. Within each of the main components of the model, more specific context-dependent variables were identified, which play a role in the relationship between and among the components of the model. These are summarized in Table 8.1.

Table 8-1: Context-Dependent Variables of Information Behaviour

\begin{tabular}{l|l|l} 
Access Parameters & Relevance/Utility Criteria & Search Strategies \\
\hline awareness & topic & channels \\
permission & level of detail & sources \\
findability & purpose & genres \\
abundance & sanction & \\
cooperation & situatedness & \\
time & specificity & \\
\hline
\end{tabular}

These variables, together with the set of ten contextual variables summarized in the previous section, are interrelated in a complex network of dependency, covariance and interaction. 
However, a strong relationship was identified between tasks and the strategy of selecting particular genres; therefore, this relationship became the focus of the next phases of research.

\subsubsection{Phase 2 Findings}

RQ3: What are the main categories and characteristics of tasks and of genres in this domain?

Two types of tasks were identified: information tasks motivated by problem situations and work tasks related to software engineering and software services consulting. Based on participants’ descriptions of their workplace activities, taxonomies of common tasks were developed. The full taxonomies are available in Chapter Five in Table 5-4. The five information tasks: fact-finding, procedural (how-to), decision-making, problem solving and learning are generic tasks; however, the set is tailored to the needs of the study population. The work task taxonomy includes eleven tasks, most of which are specific to the domain of software engineering. Some work task characteristics were identified by interview participants, and tested further in the online survey. The characteristics that were found to most differentiate between the work tasks in the taxonomy are complexity, predictability, a low-level technical orientation, and a high-level orientation.

A taxonomy of the most prevalent and well recognized genres was also developed (Chapter Five, Table 5-6). The sixteen genres are a mixture of domain-specific (e.g. design patterns) and crossdomain (e.g. FAQs) genres.

RQ4: Does a significant relationship exist between task and genre in this domain?

Findings indicate a significant relationship between these variables. Qualitative evidence from the interviews conducted in the workplace study indicated that particular genres are more useful for particular types of tasks. Further evidence for this relationship was provided by the metadata study, which found a statistically significant relationship between task and genre metadata assigned to documents used by the study population. The validation survey also found that 
usefulness scores assigned by participants to sixteen genre types varied significantly across five information task types.

RQ5: Are there patterns of association among the categories of these variables that can be identified and exploited for IR?

Findings from the analysis of IC Repository data (Chapter 5, section 5.3) indicate that patterns in task-genre associations are based on a number of underlying factors:

- Different activities and roles performed by the study population that involve particular tasks and make use of particular genres (architecture and design patterns; program managements and schedules)

- a shared level of detail and focus of tasks and genres (conceptual tasks and overview genres; specific technical tasks and factual, detail-rich genres)

Relationships between specific task and genres categories were identified and used to construct the Task - Genre Association Matrix. Because task and genre are categorical variables these associations were expressed as positive, negative and neutral relationships between pairs of task and genre types rather than numerical strengths of correlation.

\subsubsection{Phase 3 Findings}

RQ6: Does the use of task-genre relationships to filter search results improve search outcomes?

A functional prototype search system that makes use of task-genre relationships to filter search results was designed and implemented. In formal testing of the system against a Baseline version of the same system with no contextual components, the only significant difference was that searches using the Experimental system took longer. Hypotheses that the Experimental system would prove to be more effective, efficient, and more satisfying to use were not supported. Based 
on this evaluation, it can be concluded that task-genre relationships, as implemented in X-Site, did not improve search outcomes.

However, there were indications that the Experimental system has greater potential than what was realized in this study. Experimental search outcomes were less consistent than for the Baseline system: in some cases, it worked very well, and in others, it worked very badly. Follow up testing to compare retrieval precision indicated that for most search tasks, the Experimental system achieved higher precision at the top of the hitlist. This difference in precision is small, and was not observed in the user study, but it suggests that the genre filters do have some beneficial effect. Furthermore, analysis of the performance of the Experimental system indicates that the low accuracy of the genre classifier resulted in a significant portion of relevant documents being removed from the results, which was a serious handicap on the Experimental system. Therefore, further work needs to be done to improve the system before ruling out the potential positive effect of task-genre relationship in search.

\subsection{Discussion of Findings}

\subsubsection{Context and Information Behaviour}

The data collected in the initial phase of this research (Chapter 4) was a rich set of stories, examples and explanations of how and why information is sought out in the course of work practices. Informants proved to be deeply engaged in their professional work, highly conscious of the importance of information to their work, and openly frustrated by the difficulties they faced in accessing it. By focusing the interview questions on different contextual spheres, participants naturally related their behaviour to their personal, work and project contexts and identified a large number of contingencies that shape their information habits and practices. The interview data offered strong evidence that a contextual approach to IR is well-suited to this domain. 


\section{i. Contextual spheres and factors}

The four contextual spheres identified as important, person, project, task and problem, reflect the nature of work in this domain. These software engineers work alone or in small teams on welldefined and relatively short-term consultancy projects, which vary significantly from one to the next. Project parameters, such as clients, systems and tasks, as well as the roles and skill levels of the consultants are therefore very dynamic, and impact information behaviour across projects. The importance of tasks and problems is well-known as a feature of engineering (Leckie et al., 1996; Tenopir \& King, 2004); however, it is even more pronounced in this type of service work, due to the explicit representation of tasks and problems in the form of customer requirements.

Of the ten variables identified within these contextual spheres, most are well-known from previous IS\&R research. The importance of work roles, professional activities and expertise has been well-noted in the literature on the information behaviour of engineers and other professionals (Leckie et al., 1996). Expertise and familiarity are among the most commonly studied variables in IS\&R research in general (see for example, Hoelscher \& Strube, 2000), and within the engineering domain specifically (Hertzum \& Pejtersen, 2000; Holland \& Powell, 1995), and it is well-known that different levels of these variables are associated with use of different sources and information seeking habits. In this research, a clear distinction arose between the general skill level as an engineer, that is “domain expertise” (Marchinioni, 1995) and the level of knowledge with respect to a particular topic or situation, that is "topic expertise" (Allen \& Kim 2000). This distinction stood out as a function of the project-based work environment, in which both novice and seasoned employees frequently take on new and unfamiliar work.

The project variables, length and stage of project completion are temporal variables, which have been studied to a lesser extent (Ellis \& Haugan, 1997; Tushman, 1978). Both length and stage have been more widely studied as task, rather than project, variables (Savolainen, 2006; A. R. 
Taylor, Cool, Belkin, \& Amadio, 2007; Vakkari \& Hakala, 2001). This suggests that the project sphere can be considered as a meta-task, in which work tasks are nested, and for which similar attributes apply: complexity, level of detail, length, stage of completion, etc. Other project variables are more specific to this type of work, relating to the nature of the client, the system under consideration, and the software and hardware products involved. These variables serve to emphasize the dynamic nature of this type of work, which changes substantially from one situation to the next.

\section{ii. Model of Contextual Effects}

The fact that familiar contextual variables emerged from this study lends credence to these findings and serves as an indication that this search environment is representative rather than unique or atypical. However, the identification of these contextual variables is not the main contribution of this phase of the research. More interesting are the findings with respect to the effect of these variables on IS\&R behaviour, summarized in the Model of Contextual Effects on Searching and Selecting Behaviour. The model diagram (Figure 4-1, page 76) is reproduced below for reference (Figure 8-1). First, contextual variables influence accessibility, which emerges as a multi-dimensional construct including abundance, awareness, and findability of information in the workplace. This adds to earlier work that identified different aspects of accessibility in the engineering domain (Fidel \& Green, 2004), by identifying ways in which accessibility is shaped by contextual factors. Second, contextual variables influence the searcher's preferences for information characteristics. Of these, purpose, specificity and level of detail have been noted in various studies of selection critieria (Cool, Belkin, Kantor, \& Frieder, 1993; Schamber \& Barry, 1998), while situatedness and sanction are less common and likely more domain-specific. In particular, the relationship between context and level of detail is of interest, as the latter has been identified in various user-centred studies, but is not yet well-understood (Fidel \& Green, 2004). This relationship between workplace context and information characteristics 
supports other preliminary findings that engineers seek "task appropriate” information (Hertzum, 2002), that is "important to my work" (Anderson et al., 2001). Finally, the context-dependent information strategies identified here indicate that IS\&R behaviour is complex and strategic, rather than fundamentally driven by the principle of least effort, which has been cited as the major factor in IS behaviour of engineers (King et al., 1994). In this environment, knowing where, when and how to find the right kind of information for a given situation is a valuable professional skill, and one of the main contributions of a contextual search system would be to reduce dependence on this type of expert knowledge.

Figure 8-1: Model of Contextual Effects on Searching and Selecting Behaviour

Workplace Information Environment

Information Behaviour

These findings can extend existing models of information seeking to take context into greater account. The model of information seeking of professionals proposed by Leckie, Pettigrew and Sylvain (1996) includes components similar to those identified here. It consists of three interrelated components that drive the information seeking process: work roles, tasks and information needs, and three factors that influence information seeking: awareness, sources, and outcomes. However, in the Leckie et. al. model, the effect of roles and tasks is limited to shaping the 
information need, rather than influencing the information seeking process more generally. An extension of this model based on the Model of Contextual Effects presented here would recognize that contextual factors influence all aspects of the interaction, in some cases directly and in others indirectly, and that this influence changes from one situation to the next.

Another model designed to represent organization search behaviour, the Comprehensive Model of Information Seeking (CMIS) (J. D. Johnson et al., 1995) also has a structure similar to that of the Model of Contextual Effects presented here. CMIS posits that the amount of information seeking activity is a function of Antecedent variables (the imperatives that motivate a person to seek information), mediated by Information Carrier Factors, relating to characteristics of the information content and the utility (relevance, importance, topicality) of information. However, testing of this model using structured equation modeling did not show a strong relationship between antecedents and information carriers . The Model of Contextual Effects points to deficiencies in the CMIS model which may have contributed to the lack of expected correlations. First, modeling the search motivation using only personal and cognitive variables of the searcher would seem to be inadequate, rather like studying a fish out of water. Second, a definition of utility that does not take into account the suitability of information to the broader search situation is too limited. By attempting to de-contextualize search behaviour, the CMIS fails to account for much of the meaningful variation, as the authors concede in their conclusion to the paper.

\section{iii. Application of the model}

The major contribution of the Model of Contextual Effects, is that it identifies a number of avenues to promote more effective IS\&R in the workplace. The first is to find ways to mitigate the access constraints that become more pronounced under certain conditions. In particular, support tools could be developed for novice professionals, who are not yet familiar with the information and social environment in the company, for situations with tight deadlines, and for searches relating to newer products and services. The second is to find ways to exploit the 
dependencies between specific information characteristics and contextual variables. The challenge with this approach is that this is a complex, many-to-many relationship, and tools to automatically identify these types of information characteristics and contextual variables do not yet exist. The third approach is to exploit the indirect relationship between context and search strategies. The approach taken in this research focused on genre preferences as a strategy, but channel and source preferences could also be considered.

\subsubsection{Task and Genre \\ i. Tasks}

Two nested layers of task types were found to influence information behaviour in this domain: work tasks and information tasks. Recent work on the classification of tasks in information science suggests that three types of tasks are important: work tasks, information seeking tasks and information search tasks (Byström \& Hansen, 2005); however, the distinction between the two information task types did not emerge as important in this study. This may be the result of not having conducted separate studies of seeking and searching behaviour, which is a common research practice. However, based on observing information behaviour in this domain, it is debatable whether or not information seekers would be able to distinguish effectively between these types of tasks, as technologically mediated means of information seeking, through search systems, web browsing, and use of communication technologies, are no longer discrete types of activities.

The principle of division for the five information task types considered in this research is the goal, or intended information use. While goal-based approaches to classifying information seeking behaviour have been explored to a limited extent in past research (Hert \& Marchionini, 1997; Limberg, 1999), the findings of this research indicate that there is considerable untapped potential to this approach, particularly in environments which contain a wide range of different types of information objects. The small set of information tasks identified in this research: learning, fact 
finding, decision making, problem solving and procedural are sufficiently general to serve as a starting point to compiling a more extensive, cross-domain set of information tasks for further study. The value of this set of information tasks as opposed to the more commonly examined search task variables, such as browsing, navigating, information gathering, known item searching, transactional searching, etc.(see for example Broder, 2002; Kellar, Watters, \& Shepherd, 2007; Kelly et al., 2002), is that the emphasis is on the use or application of information. This approach is more applicable to full-text immersive information environments in which retrieval and use are no longer distinct activities.

Identification of work task types was based on the study population's perception of distinct types of activities, rather than on a theoretical principle of division. This approach to classifying work tasks at the level of broad professional activities is becoming more common in information behaviour studies (Lykke Nielsen, 2001; Serola, 2006). It has the advantage of being based on actual work practices and therefore carries meaning for the target population. The characteristics that best distinguish between work tasks in this domain are complexity and level of detail, of which the former has been studied extensively (Byström, 2002; Campbell, 1988; Tushman, 1978) and the latter is relatively novel and worthy of further attention (Fidel \& Green, 2004). Interestingly, most of the work tasks in the taxonomy have a similar profile: participants described them as relatively complex, low-level tasks that involve little interaction with the customer, focus on products and technology and require significant knowledge to perform. A smaller number of tasks, exemplified by project management, are more customer-focused, higher level, more predictable, and require less knowledge. There were no differences in the requirement for experience, which was high across all the tasks. This is not surprising, given the importance of hands-on learning in engineering practice (Hertzum, 2000; Paepcke, 1996). As one interview participant noted: “you cannot excel in something that you don’t have hands-on experience [in]” (P07). Although this analysis of work tasks was based on a relatively small sample and a limited 
set of task characteristics, these findings with respect to broader characteristics offer a means of generalizing results and comparing task-based IS\&R results across domains.

\section{ii. Genres}

In the course of conducting the genre analysis and developing the genre taxonomy, a number of issues arose with respect to genre classification in real-world information environments. As is generally recognized, genres are identified through some combination of form, content and purpose or function (Orlikowski \& Yates, 1994). However, it was evident from this research, that the importance of each of these features varies from one genre category to the next. Few genres in the taxonomy are strongly defined on all three facets; for example, presentations are defined primarily through form, and best practices are defined primarily through purpose. As with Web genres (Crowston \& Kwasnik, 2004), the organizational genres studied in this work were found to be related to one another in various ways. There are hierarchical relationships, in which a specific genre type, such as a lesson plan, is an instance of a broader class, such as course materials. Whole-part relationships are also rife, particularly for longer and more complex genres such as software manuals, which contain sub-sections that function as best practices, cookbooks, white papers, FAQs, etc. Finally, there are some genre categories that overlap substantially with others in terms of form, function and content, and which are difficult to distinguish solely on the basis of a feature analysis. The general challenge resulting from this complexity is that classification cannot be reduced to a static set of rules, as genre use is an evolving social practice. The complexity of genre identification in this domain supports other current research findings confirming the need for empirical, bottom-up methods applied in natural information environments (Santini, 2007).

\section{iii. Task - Genre Associations}

Task analysis and a range of other task-based methods are used commonly to study the functions and processes of the workplace in order to improve work practices and design systems (Hackos \& 
Redish, 1998). Organizational genres are studied as a means of understanding organizational structures and communication patterns and to inform the design of information systems (Honkaranta, 2003; Orlikowski \& Yates, 1994; Spinuzzi, 2003). However, the obvious connection between tasks and genres has not been the focus of attention to date. This research provides clear evidence of the relationship, suggesting that combining task-based and genre-based approaches to the study of work is likely to be beneficial.

The broad patterns of association between tasks and genres are reflective of the functional and pragmatic basis for their relationship, which is based, essentially, on the nature of activities conducted at work. Taylor (R. S. Taylor, 1991) came closest to identifying this connection, as he focused on information use rather than the more commonly studied seeking behaviour. However, Taylor focused his attention on the relationship between problem types and information characteristics, rather than at the more general level of tasks and genres. Considering the relationship between tasks and genres in the framework of the General Model of IS\&R (Ingwersen \& Järvelin, 2005), described in Chapter 2, helps to clarify its potential value (Figure 8-1).

On the right of the diagram in Figure 8-1 is the organizational and domain context out of which tasks arise and are imposed on the cognitive actors, prompting them to search for information. The genre types emerge out of the same context to add meaning and context to the information objects, which are created by cognitive actors (authors) and added to the information system. Similar intents and purposes motivate both the actors who are engaged in task-performing actions, and by the actors who are authors, and are engaged in communicative actions through the use of genres. Task - genre associations act as an implicit link between information objects and cognitive actors performing tasks, based on the common context out of which they emerge. This implicit relationship can be modeled as an explicit set of associations and embedded in an information system, which will serve to reinforce the ties between all the components of the 
IS\&R interaction. It follows, that use of task - genre associations is likely to be most effective when the contexts of document creation and of document use are overlapping, as is the case in this research setting. Similar situations certainly exist within other professional groups and communities of practice. When this is not the case, it is possible that bridges could be constructed to map tasks and genres across a contextual divide; however, further work will be needed to explore the value of task - genre associations in other types of settings.

Figure 8-2: The task-genre relationship mapped onto the General Model of IS\&R (Ingwersen \& Järvelin, 2005)

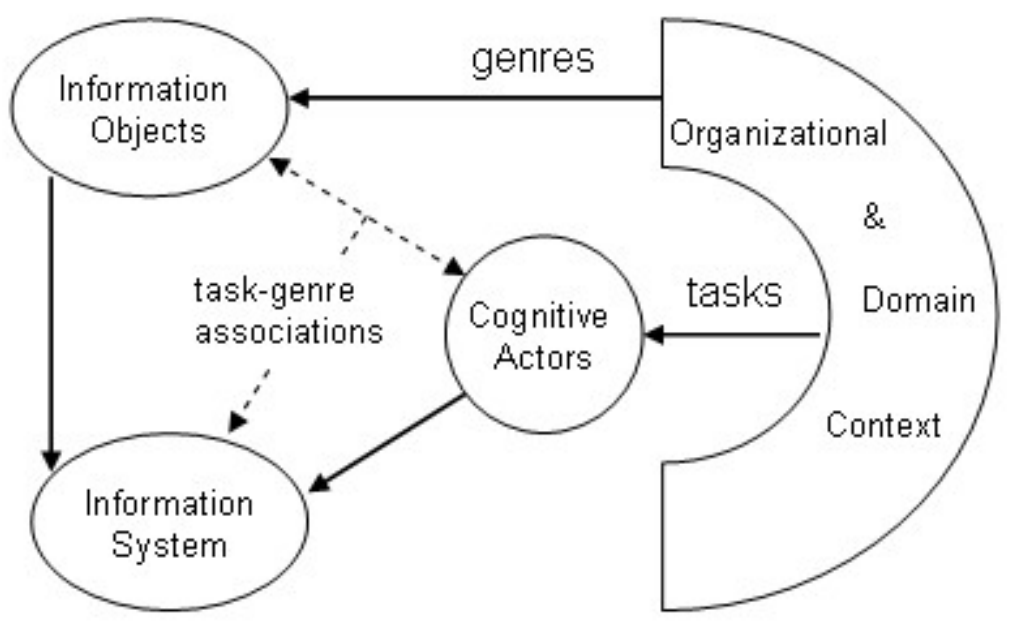

Additional characteristics of the task - genre relationship can be identified by examining patterns within the Task-Genre Association Matrix. One observation is that some tasks are positively associated with relatively few genres, and others are associated with many. The more complex tasks such as troubleshooting and performance tuning tend to be associated with a larger number of genres, which supports the finding of Byström and Järvelin (1995) that people turn to a wider variety of sources for more complex tasks. Another observation is that some genres, such as cookbooks, vary substantially in usefulness from task to task, while others are either generally useful across many tasks, or are not strongly associated with any tasks in this domain. Further, some genres are primarily associated with information tasks and others primarily with work tasks. Although the genres that vary in usefulness from task to task are most useful for applications such 
as X-Site, which seek to filter out results ill-suited to particular situations, the other types of genres may also be useful in different applications of contextual search. These observations are novel, and offer a potentially useful way of isolating small sets of genres that can make the strongest contribution to information retrieval.

A finer-grained analysis of the relationship reveals some differences between information task and work task associations with genre. The relationship between information tasks and genres is based primarily on a shared communicative purpose: an instruction manual, which is designed to teach something, is useful for someone with the information task of learning. This connection became very clear in the validation study, which found that for each information task, there was one genre, aligned in purpose, which received the highest usefulness score. There are also other types of relationships, based more on the form and content of genres, but purpose is the strongest link, which reinforces the functional approach to organizational genres introduced by Orlikowski and Yates (Orlikowski \& Yates, 1994). The relationship between work tasks and genres is more complex and indicative of the social construction of genres within communities (Spinuzzi, 2003). Some genre types seem to have developed out of a particular work-task context, either as inputs to or outputs from the work task process, and thus are suited specifically to support the task. Design patterns are associated with architecture tasks in this way. Other genres, such as cookbooks, are associated with a broader range of tasks (installation, configuration and administration) as these tasks tend to have a strong procedural component that is effectively supported by the step-by-step approach used in cookbooks. Still others are aligned based on purpose. This variation is worthy of further study, as it may be of use in considering different ways to model and make use of task - genre associations, both for applications of IR and for understanding organizational information behaviour more generally.

Results suggest that search system designers seeking to make use of task-genre relationships may wish to begin with the relationship between genres and information tasks, as defined in this 
research. This relationship seems to be stronger and more direct than the relationship with work task. Also, because the information tasks types are more generic, these relationships may be easier to apply in other domains.

\subsubsection{Implementation and Testing \\ i. Evaluation Results}

There are a number of possible reasons why the Experimental system did not perform significantly better or worse than the baseline. Given the greater variation in outcome measures in the Experimental system, it may simply have worked well enough in some cases to cancel out the effect of cases in which it worked badly. Another possibility is that the filtering mechanism of the Experimental system did increase retrieval precision, so that even when highly relevant documents were removed, the hitlist contained enough other relevant documents to compensate. So, by selecting several moderately relevant documents, rather than finding one highly relevant document, searchers were able to compensate for the effect of the filtering error. It is important to note, that regardless of the task profile chosen, the Experimental system filters out all unclassified documents in the results, which means that much of the random, information-poor content that is a major source of frustration with the corporate intranet is removed. The participant who noted, that the Experimental system retrieved, “more information, less stuff”(P28) may have been responding to the positive effect of this general content filtering.

Finally, the lack of significant differences may be a function of the method of evaluation. These searchers proved to be easily satisfied and quite experienced and adept at compensating for deficiencies in search systems. They often knew what they were looking for, and simply used the search results as stepping stone to link through to known sites, or to provide a clue or starting point that they would follow up using other channels. Given that they tended to be satisfied with only one or two relevant documents, their assessments were sensitive to a low level of precision, which both systems were able to achieve for most of the searches. 


\section{ii. System Design}

There are at least two possible approaches to improving utilization of task-genre relationships in

the X-Site system. On the one hand, the contextual components designed to collect task profiles, to classify the documents by genre and to identify task-genre associations all performed with some margin of error. Therefore, improvements to the design of these components should result in a more effective genre filter and better overall performance of the Experimental system. Further user testing and refinement of the taxonomies used in task profile selection, including the option of choosing more than one task for a given search, would improve the usability of this component. The TGAM, which is based on a combination of various secondary sources of evidence, could be improved by tuning the existing associations based on data collected from actual search behaviour, as is the practice with social and personalization systems (Brusilovsky, 2008). Finally, the genre classifier can be improved by incorporating additional linguistic and structural features of documents, based on the rapidly growing body of research in this area (See for example, Santini, 2007). However, even once these improvements have been made, some rate of error will always remain, as tasks, genres and their associations are dynamic, subjective, and fuzzy.

Given this complexity, the filter itself may be the more serious problem with this system, because it is intolerant of error. The filter approach is best suited to broad searches with a large number of relevant documents, typical of Internet searches. However, the queries formulated by this population tended to be longer and more specific, and therefore more effective (Belkin et al., 2003). Such detailed queries often retrieved only a small number of relevant documents, in which case the filter did more harm than good. A more flexible design approach would be to use evidence of task - genre associations to adjust the document weights so that more useful documents, based on genre, would be ranked more highly in the hitlist, but documents with very high topical relevance weights would also be at the top of the list, regardless of their genre. This 
is the approach we have taken in the next version of the X-Site system (Yeung, Freund, \& Clarke, 2007).

Another design issue that arose in the evaluation study is the level of system transparency and user control in the current version of X-Site. One of the central ideas behind the implementation of task - genre relationships in X-Site, was based on Belkin’s (1980) ASK concept, that the user should not be required to specify what genres they were seeking, but only to specify their task context. However, this resulted in frustration on the part of some study participants, because they did not know how the task profile functioned as a filter, and they had no control over the process. This is somewhat ironic, as most search engines function as black boxes; however by asking for extra input in the form of a task profile, the Experimental system seems to have triggered the searchers desire to understand what was going on. However, it may have been more pronounced in this study, as the population consists of software engineers who are much more aware of how these types of systems work than the average user.

The implication is that either the system should become more opaque, by finding some means to infer the task context based on implicit measures of user behaviour, or it should become more transparent. Results from earlier user studies of search systems indicate that providing greater transparency and control can be beneficial (Koenemann \& Belkin, 1996), and given the high level of knowledge and expertise of this population, this approach would likely be well-suited to this domain. Greater transparency and control could be achieved in this system quite easily, by allowing searchers to see which genres are filtered or weighted for a given task profile, and giving them the option to manually remove or add genres to the set. Having this option would allow searchers more control over clearly defined searches, with the option to use default settings for more exploratory searches. Furthermore, it could accommodate novice and senior engineers with different levels of search and domain expertise. 
While further work clearly needs to be done to refine and evaluate the task - genre approach, it is possible, based on this research, to identify conditions under which task-genre associations are most likely to make a significant contribution to search systems.

- Environments in which documents are created and used in a shared context, or are created expressly for use by a known target community;

- Environments in which one or more well-defined groups or communities of practices are active information users;

- Environments in which information seeking is prompted by the need to perform tasks (purposeful activities) and in which a variety of types of tasks are regularly performed;

- Environments in which document creation is not strictly controlled, i.e. genres are able to emerge and develop naturally;

- Environments in which the integrity of document forms and structures is preserved.

These points lead to the conclusion that this type of application is well-suited to certain types of organizational environments, which accommodate and support work groups and do not impose strict top-down controls over the information environment. Web-based communities that share information and perform common types of tasks may also prove to be well-suited, and this is currently an active area of research (Rosso, 2008; Santini, 2006; Shepherd et al., 2004).

\subsubsection{Software Engineers and Workplace Search}

A by-product of this research was a considerable amount of data on the workplace information practices of this population. This section will draw together and discuss some of these threads with an aim to highlight new perspectives raised in this research. 


\section{i. Characterizing the study population}

As a professional group, these software engineering consultants are both task-focused and service-oriented. Their main subject area interests are technology and business processes. Their work is structured through well-defined projects, and varies considerably between complex problem-solving, conceptual planning, routine technical tasks, management, teaching and mentoring activities. Because of the strong service orientation, their work is similar to other customer-facing professionals such as nurses, lawyers or doctors, as noted by Leckie et. al. (Leckie et al., 1996), more than it is to scientists or research and development engineers (Ellis \& Haugan, 1997). However, there are some important differences. This is private sector, rather than public sector work, and time and money constraints push in the direction of finding sufficient and efficient solutions to problems, rather than finding the highest quality solutions.

\section{ii. Features of the information environment}

There are a number of issues that set this organizational information environment apart from more traditional information creation and use environments. The first issue is the relatively short life-cycle of information in this environment. Within the information technology sector, products, technologies and services change very frequently, and information is quickly outdated.

Participants in the study estimated that the shelf-life of genuinely useful information in this environment was about six months to one year, which is much shorter than traditional information environments, and likely also shorter than other professional disciplines such as medicine and law. This raises some important considerations, both for information retrieval and for knowledge management work within these types of organizations, as the amount of accumulated outdated information is rapidly outpacing that which is useful. The short information life-cycle is related to another issue, which is that almost all the information objects used in this environment are in digital form. This movement away from print and paper information sources makes it much easier to create, modify, access and use information. While many former studies of engineers 
found information accessibility and availability to be key selection criteria, most of these focused on print information carriers, such as journal articles and technical reports (King et al., 1994; Orr, 1970; Pinelli et al., 1993). As predicted by Fidel and Green (2004), accessibility seems to be less of a critical issue for engineers working in a digital information environment. In this research, physical availability was more of a pre-condition than criteria for selection. Only in rare cases when consultants were working off-site and cut off from the network were they aware of information that was relevant but unavailable. However, it was much more frequent for them to be aware of relevant information that they could not find. For engineers in this digital information environment, perhaps findability is the new accessibility.

Another outcome of the move to digital information, is that information objects are more easily created and shared within the community. Much of the information used by the study population was created by other members of the group. Furthermore, within corporate environments, there are explicit mandates that information objects be created, packaged and stored for re-use (Huang, 1998). These internally-created information objects, called intellectual capital or reusable assets, exist alongside external and public information in the information environment, but are generally considered to be more valuable and useful by members of the community.

\section{iii. Situational Relevance and Information Value}

The usefulness, utility or situational relevance of information is very important in this domain. Study participants explanations of why they thought a given document was useful revealed a clear hierarchy, starting at the lowest point with documents that only contain clues or pointers, then moving up to documents that are on topic, and then to the top level at which documents are on topic and well-suited to the situation and the task at hand. In this domain, situational relevance is closely related to the concept of value-added processing (R. S. Taylor, 1986). Information is primarily viewed as an input to the process of completing tasks and projects; therefore, the value of information is directly related to the amount of processing needed to apply it to the task at 
hand. Information that is hard to extract from a document, or which needs to be interpreted, modified, verified, etc. is less useful. In fact, the most useful information object is one that can be re-used without modification, serving as a project input or deliverable. This re-using and repurposing approach to information was identified by Paepcke (Paepcke, 1996), who found that the creation of "information compounds," creative re-combinations of existing information was a major aspect of workplace information practices.

Because the applicability of information is key, information that is created in the same or in a context similar to the context of use, tends to be the most valuable. This relates to a general value hierarchy of information, which is experience-based. Interview participants commonly mentioned that their preferred method for gathering information was to work directly with the software; “you've got to go and do it a couple of times and break it until you're sure that the solution is right” (P08). However, they seldom had time for this approach. The next best thing, in order of preference, was to talk with someone who had direct experience, to read something based on direct experience, and as a last resort, to read something based on theoretical knowledge.

This observation supports Hertzum's (2002) argument that software engineers prefer to talk to colleagues, not because it is easy, but because it provides them with useful information. In fact, it became quite clear in the course of this research, that asking questions of a colleague is neither necessarily easy nor free. In this environment, everyone has looming project deadlines and more work than they can do, so each time someone asks a colleague or an expert a question, it costs the organization money. Interview participants described how they carefully cultivate contact lists, which they make use of sparingly so that they don't “abuse their privileges” and will be able to turn to these experts in times of real need. Furthermore, determining who to ask, getting access to that person, deciding whether to use the phone, email, or instant messaging, and formulating a "good" rather than a "stupid" question are complicated matters that are not tackled without good reason. 
Despite the importance of situationally relevant, experience-based information, these features are often the victims of a trade-off in this environment. Given the inability of existing search systems to filter information by the many selection criteria that are important to them, searchers are faced with the decision of how to allocate their resources. Should they spend time searching to find highly useful information objects, or search quickly, and invest time processing topically relevant but less useful information objects? The first option is more risky, since they may invest search time without finding anything more useful. The result is that these searchers often sacrifice utility and compensate by applying their professional expertise. While this may make sense at the level of the individual searcher, it is inefficient for the organization as a whole, as it results in repetition and wasted resources (Feldman \& Sherman, 2003).

\subsection{Summary}

This chapter has summarized and discussed the findings of this research in four sections: context and information behaviour, task-genre relationships, implementation and testing, and software engineers and workplace search. What has emerged is a detailed picture of the influence of context on information behaviour in this domain. Some findings reinforce what is already known about the information behaviour of professionals in the workplace, in particular the importance of roles, tasks, and expertise, but some findings are novel and worthy of further attention.

With respect to tasks and genres, results provide strong evidence that a relationship between task and genre exists, as well as identifying a number of dimensions and characteristics of that relationship. However, findings show that the implementation of task-genre relationships in the X-Site search engine did not improve search outcomes. Tasks and genres are not objective, easily defined or measures features of workplace environments, and more work needs to be done to reach a robust characterization and implementation of their relationships. 
However, the experience did provide valuable insights, both into the performance of the search system, which will lead to improvements in future versions, and into the search behaviour of the population. Developing tools for this type of knowledgeable, technologically sophisticated and motivated searcher is not a simple matter, given their desire to understand and control what the search engine is doing, together with their unwillingness to engage in lengthy interactions with the system or the information objects retrieved. What these searchers are most interested in and knowledgeable about, is their work, which is something that information scientists, system designers and librarians have yet to come to grips with. By focusing on context, practice and use in this research, I believe that we have taken one step closer to understanding the searcher's perspective. 


\section{Conclusion}

\subsection{Overview}

This chapter will summarize the contributions of this work, discuss some limitations, and propose directions for future research.

\subsection{Contributions}

This research focused on workplace search within a community for whom searching is not about entertainment or trivia, but rather is, "the heartbeat of what we do" (P13). It contributes to a better understanding of the problems and issues of workplace search and offers a theoretical framework as well as some practical steps towards extending IR systems to better address those problems.

\subsubsection{Model}

The model of contextual effects in IS\&R presented in Chapter Four makes a number of contributions to research in this field. It offers a novel perspective on IS\&R by focusing on contextual effects as drivers of information behaviour. Based on real-world data, it identifies a set of contextual variables that have strong effects on searching and selecting behaviour in the workplace, and describes the nature of their impact. The modeling of context and contextual effects for use in IR is an area of increasing interest, so there is significant value in this empirically determined, albeit domain-specific, model. 
The model is valuable for its integrated approach to information seeking and information searching activities. It shows how information seeking strategies are employed purposefully as a means of overcoming information access constraints and obtaining situationally relevant information. The model suggests various ways in which context can be exploited in IR systems: by incorporating relationships between context and information characteristics into matching algorithms, by providing context-dependent support for information seeking strategies, by compensating for contextually determined access constraints, and by automating information strategies based on variations in context. By identifying and characterizing the effect of specific contextual factors, this model fleshes out some of the elements of the framework developed by Ingwersen and Järvelin (2005), and provides directions for further study.

\subsubsection{Situated Relevance}

This research contributes to our understanding of the role of situational relevance in IS\&R. In this environment, searchers make strategic information seeking decisions in order to obtain information that is suited to the situation. The less effort required to make use of an information object and apply it to the task at hand, the greater its situational relevance and value. Genre emerges as a strategic means of predicting the situational relevance of documents within the workplace. A practical and unique contribution of this work, is the identification of a set of nontopical information characteristics that software engineers use as situated relevance criteria (level of detail, specificity, situatedness, sanction and purpose). These information characteristics are among those that next generation workplace IR systems need to be able to detect and exploit in order to move beyond current levels of retrieval precision.

\subsubsection{Methods}

A number of innovative research methods were employed over the course of this research. These were used in response to the challenges of conducting research in the workplace and of evaluating a contextual IR system in context. The IC Repository metadata analysis (Chapter 5) is both a 
creative use of "found" data of the sort that is likely to be available in many organizational environments, and a novel application of a statistical technique to explore categorical data. The evaluation study (Chapter 7) employed a remote testing setup using NetMeeting, which allowed participants located throughout North America to participate in the experiment without mounting the system or the study instruments on the Internet. Finally, the test collection (Chapter 7) that was created with the data from the user study is an interesting hybrid approach to IR system evaluation, with potential for further refinement and application. The use of these methods and their presentation in this dissertation will be of use as a guide to researchers dealing with similar types of data and methodological challenges.

\subsubsection{Tasks and Genres}

This is the first major study of the relationship between tasks and genres in a workplace information environment. The topic is of potential interest in a range of fields and this work is likely to prompt further research in this area. A number of specific contributions to future research were made:

- A significant relationship between task and genre was demonstrated;

- Work roles and level of detail were identified as factors that shape the task-genre relationship;

- A user-specified task profile, consisting of two levels of task types, a work task and an information task, was developed as a simple framework for representing the task context of a search. The profile can applied in any search environment by customizing the task taxonomies; 
- The bottom-up approach to genre classification used in this research serves as a practical model and proof of concept for the construction of genre taxonomies for use in workplace IR systems;

- The matrix of task-genre associations developed in this research offers a simple framework for representing and exploiting the relationship between tasks and genres.

\subsubsection{Contextual Search}

This research has demonstrated that there is value in viewing IS\&R from a pragmatic perspective, as a purposeful and situated workplace activity. The focus on a population of searchers with a clear need for improved search tools capable of supporting professional work activities stands in the face of the perception that high-recall, general purpose Internet search tools have solved the search problem. X-Site is one of the few implementations of contextual search based on empirical evidence of information behaviour within a particular domain. While there is certainly room to improve the system, it demonstrates that evidence of contextual effects on IS\&R can be incorporated and exploited in retrieval systems.

\subsection{Limitations}

This research was conducted within a single organization among a large, but cohesive group of employees. This limited scope was necessary to focus on IS\&R in context, but it does limit the claims to external validity that can be made. Findings with respect to the information issues faced within this organization and the information behaviour and workplace practices of the study population suggest that there are strong parallels with other large organizations and with other groups of engineers and professionals; however, these parallels cannot be assumed without further validation of findings in other environments. 
One of the goals of this research was to bridge the gap between information seeking and information retrieval research by applying the results of an information behaviour study to the design of an IR system. There were two main challenges associated with this goal. The first is that it meant moving from a qualitative to a quantitative paradigm. The result is that considerable interpretation was involved in producing the data used to implement task-genre relationships. Although the existence of task-genre relationships is not in question, the data on specific relationships is context specific and subject to interpretation, thus further empirical testing and tuning of these relationships would increase their validity. The second challenge is that a considerable amount of ground was covered rather quickly in moving from the initial exploratory study to the final system evaluation. Although not possible in the framework of this work, additional time spent on the implementation and testing of the contextual components of X-Site would have been valuable, as the system proved to lack robustness in evaluation.

Another limitation of this work is that the model of IS\&R that emerged is relatively static. Contextual effects are modeled at the level of an individual search session, but not at the level of the interactive process of searching that occurs within each search session. This is not due to a lack of recognition of the human-computer and human-information interaction that takes place during searching, but rather represents a choice to emphasize the relatively static effects of workplace context, rather than the more dynamic, moment-by-moment effects of the search process. A more complete model of contextual effects would need to be extended to include the interactive aspect of IS\&R.

\subsection{Future Work}

\subsubsection{System Enhancements}

The first steps required to move forward with this research are to implement improvements to the X-Site system. Work has already been done to move from a filtering to a ranking model, based on 
a theoretical framework that supports the incorporation of genre weights into the retrieval algorithm (S. Robertson, Zaragota, \& Taylor, 2004; Yeung \& Clarke, 2007; Yeung, Clarke, \& Büttcher, 2007). A new version of the system, using genre weighting and incorporating some of the system improvements suggested in the evaluation study is already available (Yeung, Freund \& Clark, 2007), but has not yet undergone formal evaluation. Two other major areas of improvement still need to be addressed. The first is to improve the genre classifier by extending the feature set beyond a "bag of words" to incorporate additional linguistic and structural features of documents. The second major improvement is to improve the accuracy and validity of the TGAM by tuning it to reflect actual system use. This step requires deployment of X-Site within the organization in order to collect evidence of the genres selected by searchers with specific task profiles.

\subsubsection{Generalization of System}

One of the pressing questions raised by this research, is to what extent the models and techniques presented here can be applied in other contexts. One interesting avenue for future research is the extension of the current system for use by other professionals within the same organization. By identifying the tasks and genres relevant for other groups working with many of the same documents, including the development, marketing, and technical support teams, the system may be capable of providing two levels of contextual ranking, by work role, and by search situation. Other environments in which it would be valuable to test this approach to contextual search are other professional work environments and among virtual communities using shared Internet resources. Digital genres are a prevalent feature of the Internet as well as organizational environments, so the latter case would serve as an interesting test of the breadth of applicability of task-genre relationships in search. 


\subsubsection{Theory Building}

This work took an empirical, bottom-up approach to the identification and implementation of task-genre relationships. However, in the course of the research, a number of general characteristics and patterns were identified, which may prove useful in developing a theoretical model of task-genre relationships. Future work will include more focused studies of the factors that influence task-genre relationships and the characteristics that distinguish between tasks and between genres with the goal of extending our theoretical understanding of this phenomenon.

\subsection{Summary}

The challenges of finding information in the workplace and the limitations of keyword-based matching in IR systems were the starting-points for this research. In seeking a path from aboutness to usefulness, I explored the information environment and identified the role that contextual factors play in shaping information seeking and selecting behaviour within it. The clearest path through the forest proved to be the relationship between tasks and genres, a pragmatic and situated relationship that is grounded in the work activities and information practices of this community. The major contribution of this work is the identification and exploration of that relationship, which represents one approach to extending the traditional system-based and user-centred models of IR towards broader, more contextual models, capable of providing information relevant to the situation at hand. 


\section{References}

Algon, J. (1999). The effect of task on the information-related behaviors of individuals in a workgroup environment (Doctoral dissertation, Rutgers - The State University of New Jersey New Brunswick): Dissertation Abstracts International.

Allan, J., Aslam, J., Belkin, N. J., Buckley, C., Callan, J., Croft, B., et al. (2003). Challenges in information retrieval and language modeling: Report of a workshop held at the Center for Intelligent Information retrieval, University of Massachusetts, Amherst, September 2002. SIGIR Forum, 37(1), 31-47.

Allen, B. (1996). Information tasks: toward a user-centered approach to information systems. San Diego: Academic Press.

Allen, B., \& Kim, K.-S. (2000). Person and context in information seeking: interactions between cognitive and task variables. The New Review of Information Behaviour Research, 1, 116.

Allen, D., \& Wilson, T. D. (2003). Information overload: context and causes. The New Review of Information Behaviour Research, 4, 31-44.

Allen, T. J. (1977). Managing the flow of technology. Cambridge, Mass.: MIT Press.

Anderson, C. J., Glassman, M., McAfee, R. B., \& Pinelli, T. (2001). An investigation of factors afffecting how engineers and scientists seek information. Journal of Engineering and Technology Management, 18(2), 131-155.

Askehave, I., \& Nielsen, A. E. (2005). What are the characteristics of digital genres? - Genre theory from a multi-modal perspective. In Proceedings of the 38th Hawaii International Conference on System Sciences (Vol. 4): IEEE Computer Society.

Askell-Williams, H., \& Lawson, M. J. (2004). A correspondence analysis of child-care students' and medical students' knowledge about teaching and learning. International Education Journal, 5(2), 176-204.

Baeza-Yates, R., \& Ribeiro-Neto, B. (1999). Modern information retrieval. New York: AddisonWesley.

Bailey, P., Agrawal, D., \& Kumar, A. (2007). TREC 2007 enterprise track at CSIRO. In Proceedings of the Sixteenth Text REtrieval Conference (TREC 2007). Gaithersburg, MD: NIST.

Bales, S., \& Wang, P. (2005). Consolidating user relevance criteria: A meta-ethnography of empirical studies. In Proceedings of the 68th Annual Meeting of the American Society for Information Science and Technology. Silver Spring, MD: ASIS\&T.

Barry, C. L. (1994). User-defined relevance criteria: An exploratory study. Journal of the American Society for Information Science, 45, 149-159.

Bazerman, C., Little, J., \& Chavkin, T. (2003). The production of information for genred activity spaces. Written Communication, 20(4), 455-477. 
Belkin, N. J. (1980). Anomalous states of knowledge as a basis for information retrieval. Canadian Journal of Information Science, 5, 133-143.

Belkin, N. J. (1990). The cognitive viewpoint in information science. Journal of Information Science, 16(1), 11-15.

Belkin, N. J., Cool, C., Kelly, D., Kim, G., Kim, J.-Y., Lee, H.-J., et al. (2003). Query length in interactive information retrieval. In Proceedings of the 26th Annual International ACM SIGIR Conference on Research and Development in Information Retrieval (pp. 205-212). New York, NY: ACM.

Belkin, N. J., Muresan, G., \& Zhang, X.-M. (2004, July ). Investigating the effect of the use of user's context on IR performance. Paper presented at the Workshop on Information Retrieval in Context (IRiX) at SIGIR 2004, Sheffield, England.

Belkin, N. J., Oddy, R. N., \& Brooks, H. M. (1982a). ASK for information retrieval: Part I: background and theory. Journal of Documentation, 38(2), 61-71.

Belkin, N. J., Oddy, R. N., \& Brooks, H. M. (1982b). ASK for information retrieval: Part II. Results of a design study. Journal of Documentation, 38(3), 145-164.

Belkin, N. J., Seeger, T., \& Wersig, G. (1983). Distributed expert problem treatment as a model for information analysis and design. Journal of Information Science, 5(5), 153-167.

Bell, D., \& Ruthven, I. (2004). Searcher's assessments of task complexity for web searching. In S. McDonald \& J. Tait (Eds.), Advances in Information Retrieval, 26th European Conference on IR Research, ECIR 2004, Sunderland, UK, April 5-7, 2004, Proceedings (pp. 57-71): Springer.

Beyer, H., \& Holzblatt, K. (1998). Contextual design : a customer-centered approach to systems designs. San Diego, CA: Academic Press.

Bodker, S. (1991). Through the interface: A human activity approach to user interface design. Hillsdale, NJ: Lawrence Erlbaum Ass.

Borlund, P. (2003a). The concept of relevance in IR. Journal of the American Society for Information Science, 54(10), 913-925.

Borlund, P. (2003b). The IIR evaluation model: a framework for evaluation of interactive information retrieval systems. Journal, 8(3). Retrieved from http://informationr.net/ir/83/paper152.html

Bretan, I., Dewe, J., Hallberg, A., Wolkert, N., \& Karlgren, J. (1998). Web-specific genre visualization. In H. A. Maurer \& R. G. Olson (Eds.), Proceedings of WebNet 98 - World Conference on the WWW and Internet \& Intranet, Orlando, Florida, USA, November 712, 1998: AACE.

Broder, A. (2002). A taxonomy of Web search. SIGIR Forum, 36(2), 3-10.

Brusilovsky, P. (2008). Social information access: the other side of the social web. In SOFSEM 2008: Theory and Practice of Computer Science (Vol. 4910, pp. 5-22). Berlin: Springer. 
Bryce, A. (1997). Information needs: a person-in-situation approach. Paper presented at the Proceedings of an international conference on Information seeking in context.

Byström, K. (2002). Information and information sources in tasks of varying complexity. Journal of the American Society for Information Science, 53(7), 581-591.

Byström, K., \& Hansen, P. (2005). Conceptual framework for tasks in information studies. Journal of the American Society for Information Science and Technology, 56(10), 10501061.

Byström, K., \& Järvelin, K. (1995). Task complexity affects information seeking and use. Information Processing \& Management, 31(2), 191-213.

Campbell, D. J. (1988). Task complexity; a review and analysis. The Academy of Management Review, 13(1), 40-52.

Case, D. O. (2002). Looking for information: A survey of research on information seeking, needs, and behavior. San Diego: Academic Press.

Case, D. O. (2006). Information seeking. In B. Cronin (Ed.), Annual Review of Information Science and Technology (Vol. 40). Medford, NJ: Information Today.

Chang, S. L., \& Yee, Y. (2001). Conceptualizing context and its relationship to the information behaviour in dissertation research process. The New Review of Information Behaviour Research, 2, 43-78.

Choo, C. W., Detlor, B., \& Turnbull, D. (2000a). Information seeking on the Web - an integrated model of browsing and searching. First Monday, 5(2).

Choo, C. W., Detlor, B., \& Turnbull, D. (2000b). Web work: information seeking and knowledge work on the World Wide Web. Amsterdam: Kluwer Academic.

Choo, C. W., Detlor, B., \& Turnbull, D. (2000c). Working the web: an empirical model of web use. In Proceedings of the 33rd Annual Hawaii International Conference on Systems Science (HICSS-33), 4-7 January, 2000, Maui, Hawaii.: IEEE Computer Society.

Cleverdon, C. W. (1960). ASLIB-Cranfield Research Project: Report on the first stage of an investigation into the comparative efficiency of indexing systems. Cranfield: The College of Aeronautics.

Cleverdon, C. W., Mills, J., \& Keen, E. M. (1966). Factors determining the performance of indexing systems (Vol. 2). Cranfield: ASLIB-Cranfield Project.

Cool, C. (1997). Situation assessment in information retrieval interaction (Doctoral dissertation, Rutgers - The State University of New Jersey - New Brunswick): Dissertation Abstracts International 58/07, AAT 9800243.

Cool, C. (2001). The concept of situation in information science. Annual Review of Information Science and Technology, 35, 5-42. 
Cool, C., Belkin, N. J., Kantor, P. B., \& Frieder, O. (1993). Characteristics of texts affecting relevance judgments. In M. Williams (Ed.), Proceedings of the 14th National Online Meeting (pp. 77-84). Medford, NJ: Learned Information Inc.

Cool, C., \& Spink, A. (2002). Issues of context in information retrieval (IR): an introduction to the special issue. Information Processing \& Management, 38(5), 605-611.

Cooper, W. S. (1971). A definition of relevance for information retrieval. Information Storage and Retrieval, 7(1), 19-37.

Cosijn, E. (2006). Relevance judgements within the context of work tasks. In I. Ruthven, P. Borlund, P. Ingwersen, N. J. Belkin, A. Tombros \& P. Vakkari (Eds.), Proceedings of the 1st International Symposium on Information Interaction in Context, (IIiX 2006), Copenhagen, Denmark, 18-20 October (pp. 33-49). New York, NY: ACM.

Cosijn, E., \& Ingwersen, P. (2000). Dimensions of relevance. Information Processing \& Management, 36, 533-550.

Craswell, N., de Vries, A., \& Soboroff, I. (2005). Overview of the TREC 2005 enterprise track. In E. M. Voorhees \& L. P. Buckland (Eds.), Proceedings of the fourteenth Text Retrieval Conference (TREC 2005). Gaithersburg, MD: NIST.

Creswell, J. W. (2003). Research Design: qualitative, quantitative and mixed methods approaches (2nd ed.). Thousand Oaks, CA.

Crowston, K., \& Kwasnik, B. (2004). A framework for creating a faceted classification of genres: addressing issues of multidimensionality. In Proceedings of the 37th Hawaii International Conference on System Sciences (HICSS-37), 5-8 January, Big Island, Hawaii (Vol. 4): IEEE Computer Society.

Crowston, K., \& Williams, M. (2000). Reproduced and emergent genres of communication on the World Wide Web. The Information Society, 16(3), 201-215.

Davenport, E. (2002). Organizational knowledge and communities of practice. Annual Review of Information Science and Technology, 36, 171-227.

Davenport, T. (1997). Information ecology: mastering the information and knowledge environment. New York: Oxford University Press.

de Mey, M. (1980). The relevance of the cognitive paradigm for information science. In O. Harbo (Ed.), Theory and Application of Informaiton Research (pp. 48-61). London: Mansell.

Dervin, B. (1997). Given a context by any other name: methodological tools for taming the unruly beast. In P. Vakkari, R. Savolainen \& B. Dervin (Eds.), Information Seeking in Context (pp. 13-38). London: Taylor Graham.

Dervin, B. (1999). On studying information seeking methodologically: the implications of connecting metatheory to method. Information Processing \& Management, 35, 727-750.

Dervin, B., \& Dewdney, P. (1986). Neutral questioning: a new approach to the reference interview. $R Q, 25,506-513$. 
Dervin, B., \& Nilan, M. (1986). Information needs and uses. Annual Review of Information Science and Technology, 21, 3-33.

Dewdney, N., VanEss-Dykema, C., \& MacMillan, R. (2001). The form is the substance: classification of genres in text, Proceedings of the ACL Workshop on Human Language Technology and Knowledge Management Available from http://www.elsnet.org/km2001/dewdney.pdf

Dillon, A. (2004). Designing usable electronic text (2nd ed.). London: CRC Press.

Drucker, P. F. (1959). Landmarks of tomorrow: a report on the new "post-modern" world. New Brunswick, USA: Transaction Publishers.

Duan, H., Zhou, Q., Lu, Z., Jin, O., Bao, S., Cao, Y., et al. (2007). Research on enterprise track of TREC 2007 at SJTU APEX Lab. In Proceedings of the Sixteenth Text REtrieval Conference (TREC 2007). Gaithersburg, MD: NIST.

Edmunds, A., \& Morris, A. (2000). The problem of information overload in business organizations: a review of the literature. International Journal of Information Management, 20(1), 17-28.

Ellis, D. (1992). The physical and cognitive paradigms in information retrieval research. Journal of Documentation, 48(1), 45-64.

Ellis, D., \& Haugan, M. (1997). Modelling the information seeking patterns of engineers and research scientists in an industrial environment. Journal of Documentation, 53(4), 384403.

Fagin, R., Kumar, R., McCurley, K. S., Novak, J., Sivakumar, D., Tomlin, J. A., et al. (2003). Searching the workplace web. In Proceedings of the 2003 International World Web Conference (WWW '03) (pp. 366-375): ACM Press.

Feldman, S. (2004). The high cost of not finding information. KM World, 13.

Feldman, S., \& Sherman, C. (2003). The high cost of not finding information (No. 29127): IDC.

Fidel, R., \& Green, M. (2004). The many faces of accessibility: engineers' perception of information sources. Information Processing \& Management, 40, 563-581.

Fidel, R., \& Pejtersen, A. M. (2004). From information behaviour research to the design of information systems: the Cognitive Work Analysis framework. Journal, 10(1). Retrieved from http://informationr.net/ir/10-1/paper210.html

Fidel, R., Pejtersen, A. M., Cleal, B., \& Bruce, H. (2004). A multidimensional approach to the study of human-information interaction: a case study of collaborative information retrieval. Journal of the American Society for Information Science and Technology, 55(11), 939-953.

Firth, D. R. (2003). Genre analysis in information systems research. Journal of Information Technology Theory and Application, 5(3), 63-77. 
Forward, A., \& Lethbridge, T. C. (2002). The relevance of software documentation, tools and technologies: a survey. In Proceedings of the 2002 ACM Symposium on Document Engineering, McLean, Virginia, USA (pp. 26-33). New York, NY: ACM.

Glover, E. J., Lawrence, S., Gordon, M. D., Birmingham, W. P., \& Giles, C. L. (2001). Web search -- your way. Communications of the ACM, 44(12), 97-102.

Greenacre, M. J. (1984). Theory and applications of correspondence analysis. London: Academic Press.

Greenacre, M. J. (2007). Correspondence analysis versus spectral mapping/weighted log-ratio analysis. Paper presented at the JOCLAD 2007 International Conference of Portuguese Classification and Data Analysis Group.

Greene, J. C., \& Caracelli, V. J. (Eds.). (1997). Advances in mixed-methods evaluation: the challenges and benefits of integrating diverse paradigms. San Francisco: Jossey-Bass.

Hackos, J. T., \& Redish, J. C. (1998). User and task analysis for interface design. New York: John Wiley \& Sons.

Hansen, P. (2005). Work task-oriented studies of IS\&R processes. In K. Fisher, S. Erdelez \& L. McKechnie (Eds.), Theories of Information Behaviour (pp. 392-396). Medford, NJ: ASIST.

Hawking, D. (2004). Challenges in enterprise search. In K.-D. Schewe \& H. Williams (Eds.), Proceedings of the Fifteenth Australasian Database Conference (ADC 2004), Dunedin, NZ (Vol. 27, pp. 15-24): Australian Computer Society.

Hawking, D. (2005). Context in enterprise search and delivery. Paper presented at the ACM SIGIR 2005 Workshop on Information Retrieval in Context (IRiX).

He, D., \& Demner-Fushman, D. (2003). HARD experiment at Maryland: from need negotiation to automated HARD process. In Proceedings of the Twelfth Text REtrieval Conference. Gaithersburg, MD: NIST.

Hert, C. A., \& Marchionini, G. (1997). Seeking statistical information in federal websites: users, tasks, strategies and design recommendations. Final Report to the United States Bureau of Labor Statistics.

Hertzum, M. (2000). People as carriers of experience and sources of commitment: information seeking in a software design project. New Review of Information Behaviour Research, 1, 135-149.

Hertzum, M. (2002). The importance of trust in software engineers' assessment and choice of information sources. Information and Organization, 12(1), 1-18.

Hertzum, M., Anderson, H. H. K., Anderson, V., \& Hansen, C. B. (2002). Trust in information sources: seeking information from people, documents, and virtual agents. Interacting with Computers, 14, 575-599. 
Hertzum, M., \& Pejtersen, A. M. (2000). The information-seeking practices of engineers: searching for documents as well as for people. Information Processing \& Management, 36(5), 761-778.

Hjorland, B. (2002). Domain analysis in information science: eleven approaches. Journal of Documentation, 58(4), 422-462.

Hjorland, B., \& Albrechtsen, H. (1995). Toward a new horizon in information science: domain analysis. Journal of the American Society for Information Science, 46(6), 400-425.

Hoelscher, C., \& Strube, G. (2000). Web search behavior of Internet experts and newbies. Computer Networks, 33, 337-346.

Holland, M. P., \& Powell, C. K. (1995). A longitudinal survey of the information seeking and use habits of some engineers. College and Research Libraries, 14(4), 196-204.

Honkaranta, A. (2003). Developing document and content management in enterprises using a "genre lens". In O. Camp, J. Filipe, S. Hammoudi \& M. Piattini (Eds.), Proceedings of the 5th International Conference on Enterprise Information Systems, Angers, France, April 23-26, 2003 (Vol. 3, pp. 334-340): Escola Superior de Tecnologia de Setubal

Hsieh-Yee, I. (2001). Research on Web search behavior. Library and Information Science Research, 23, 167-185.

Huang, K. T. (1998). Capitalizing on intellectual assets. IBM Systems Journal, 37(4).

Ingwersen, P. (1982). Search procedures in the library analysed from the cognitive point of view. Journal of Documentation, 38, 165-191.

Ingwersen, P. (1984). Psychological aspects of information retrieval. Social Science Information Studies, 4(2/3), 83-95.

Ingwersen, P. (1992). Information retrieval interaction. London, UK: Taylor Graham.

Ingwersen, P. (1996). Cognitive perspectives of information retrieval. Journal of Documentation, 52(1), 3-50.

Ingwersen, P. (2005). Selected variables for IR interaction in context: Introduction to IRiX SIGIR 2005 Workshop. Paper presented at the ACM SIGIR 2005 Workshop on Information Retrieval in Context (IRiX).

Ingwersen, P., \& Järvelin, K. (2005). The turn: Integration of information seeking and retrieval in context. Berlin: Springer.

Ingwersen, P., Järvelin, K., \& Belkin, N. J. (2005). Preface In B. Larsen (Ed.), Proceedings of the ACM SIGIR 2005 Workshop on Information Retrieval in Context (IRiX). Salvador, Brazil.

Järvelin, K. (1986). On information, information technology and the development of society: an information science perspective. In P. Ingwersen, L. Kajberg \& M. Pejtersen (Eds.), Information technology and information use: towards a unified view of information and information technology (pp. 35-55). London: Taylor Graham. 
Järvelin, K., \& Ingwersen, P. (2004). Information seeking research needs extension towards tasks and technology. Journal, 10(1). Retrieved from http://InformationR.net/ir/101/paper212.html

Järvelin, K., \& Wilson, T. D. (2003). On conceptual models for information seeking and retrieval research. Journal, 9(1). Retrieved from http://informationr.net/ir/9-1/paper163.html

Joachims, T. (1998). Text categorization with support vector machines: Learning with many relevant features. In C. Nedellac \& C. Rouveirol (Eds.), Proceedings of the European Conference on Machine Learning (ECML) (pp. 137-142). Berlin: Springer.

Joachims, T. (1999). Making large-scale SVM learning practical. In B. Schölkopf, C. Burges \& A. Smola (Eds.), Advances in Kernel Methods - Support Vector Learning: MIT-Press.

Johnson, J. D. (2003). On contexts in information seeking. Journal of the American Society for Information Science, 39(5), 735-760.

Johnson, J. D., Donohue, W. A., Atkin, C., K., \& Johnson, S. (1995). A comprehensive model of infomation seeking. Science Communication, 16(3), 274-303.

Johnson, R. B., \& Onwuegbuzie, A. J. (2004). Mixed methods research: a research paradigm whose time has come. Educational Researcher, 33(7), 14-26.

Joho, H., \& Jose, J. M. (2006). Slicing and dicing the information space using local contexts. In I. Ruthven, P. Borlund, P. Ingwersen, N. J. Belkin, A. Tombros \& P. Vakkari (Eds.), Proceedings of the 1st International Symposium on Information Interaction in Context, (IIiX 2006), Copenhagen, Denmark, 18-20 October (pp. 111-126). New York, NT: ACM.

Jose, J., \& van Rijsbergen, C. J. (2005). Workshop on information retrieval in context: Report. Glasgow: University of Glasgow.

Kari, J., \& Savolainen, R. (2003). Towards a contextual model of information seeking on the Web. The New Review of Information Behaviour Research, 4, 155-175.

Karlgren, J. (1999). Non-topical factors in information access. In P. DeBra \& J. J. Leggett (Eds.), Proceedings of WebNet 99 - World Conference on the WWW and Internet, Honolulu, Hawaii, USA, October 24-30 (Vol. 1, pp. 27-31). Charlottesville, VA: Association for the Advancement of Computing in Eduction (AACE).

Kekäläinen, J., \& Järvelin, K. (2002). Evaluating information retrieval systems under the challenges of interaction and multidimensional dynamic relevance. In H. Bruce, R. Fidel, P. Ingwersen \& P. Vakkari (Eds.), Proceedings of the 4th CoLIS Conference (pp. 253270). Greenwood Village, CP.: Libraries Unlimited. .

Kellar, M., Watters, C., \& Shepherd, M. (2007). A field study characterizing Web-based information-seeking tasks. Journal of the American Society for Information Science \& Technology, 58(7), 999-1018.

Kelly, D. (2006). Measuring online information seeking context, part 1: background and method. Journal of the American Society for Information Science and Technology, 57(13), 17291739. 
Kelly, D., Murdock, V., Yuan, X., Croft, W. B., \& Belkin, N. J. (2002). Features of documents relevant to task and fact-oriented questions. In Proceedings of the Eleventh International Conference on Information and Knowledge Management (pp. 645-647). New York, NY: ACM.

Kim, S., \& Soergel, D. (2005). Selecting and measuring task characteristics as independent variables. In Proceedings of the 68th Annual ASIS\&T Meeting, Charlotte, NC (Vol. 42). Medford, NJ: Information Today.

King, D. W., Casto, J., \& Jones, H. (1994). Communication by engineers: a literature review of engineers' information needs, seeking processes, and use. Washington, D.C.: Council on Library Resources.

Kirsh, D. (2000). A few thoughts on cognitive overload. Intellectica, 30, 19-51.

Koenemann, J., \& Belkin, N. J. (1996). A case for interaction: A study of interactive information retrieval behavior and effectiveness. In R. Bilger, S. Guest \& M. J. Tauber (Eds.), CHI '96: Proceedings of the SIGCHI conference on Human factors in computing systems (pp. 205-212). New York, NY: ACM.

Kuhlthau, C. C. (1993). A principle of uncertainty for information seeking. Journal of Documentation, 49(4), 339-355.

Kushmerick, N., \& Finn, A. (2006). Learning to classify documents according to genre. Journal of the American Society for Information Science, 57(11), 1506-1518.

Kwasitsu, L. (2003). Information-seeking behaviour of design, process, and manufacturing engineers. Library and Informaiton Science Research, 25(4), 459-476.

Leckie, G., Pettigrew, K. E., \& Sylvain, C. (1996). Modeling the information-seeking of professionals: a general-model derived from research on engineers, health-care professionals, and lawyers. Library Quarterly, 66(2), 161-193.

Lee, Y.-B., \& Myaeng, S. H. (2004). Automatic identification of text genres and their roles in subject-based categorization. In Proceedings of the 37th Hawaii International Conference on System Sciences (HICSS-37), 5-8 January, Big Island, Hawaii (Vol. 4): IEEE Computer Society

Li, Y. (2004). Task type and a faceted classification of tasks. Paper presented at the American Society of Information Science and Technology, Providence, RI.

Limberg, L. (1999). Experiencing information seeking and learning: a study of the interaction between two phenomena. Journal, 5(1). Retrieved from http://informationr.net/ir/51/paper68.html

Lin, J., \& Demner-Fushman, D. (2006). The role of knowledge in conceptual retrieval: a study in the domain of clinical medicine. In S. Dumais, E. Efthimiadis, D. Hawking \& K. Järvelin (Eds.), Proceedings of the 29th Annual International ACM SIGIR conference on Research and Development in Information Retrieval, University of Washington, Seattle, WA (pp. 99-106). New York, NY: ACM. 
Lohr, S. (2007, December 20). Is Information Overload a \$650 Billion Drag on the Economy? The New York Times. Retrieved February 20, 2008, from http://bits.blogs.nytimes.com/2007/12/20/is-information-overload-a-650-billion-drag-onthe-economy/

Lykke Nielsen, M. (2001). A framework for work task based thesaurus design. Journal of Documentation, 57(6), 774-797.

MacMullin, S. E., \& Taylor, R. S. (1984). Problem dimensions and information traits. The Information Society, 3(1), 91-111.

Meyer zu Eissen, S., \& Stein, B. (2004). Genre classification of web pages: User study and feasibility analysis. In S. Biundo, T. Fruhwirth \& G. Palm (Eds.), KI 2004: Advances in Artificial Intelligence (pp. 256-269.). Berlin: Springer.

Mizzaro, S. (1998). How many relevances in information retrieval? Interacting with Computers, 10(3), 305-322.

Muresan, G., Smith, C. L., Cole, M., Liu, L., \& Belkin, N. J. (2006). Detecting document genre for personalization of information retrieval. In Proceedings of the 39th Hawaii International International Conference on Systems Science (HICSS-39 2006), 4-7 January 2006, Kauai, HI: IEEE Computer Society.

Nordlie, R. (1999). User revealment - A comparison of initial queries and ensuing question development in online searching and in human reference interactions. In F. Gey (Ed.), Proceedings of the 22nd Annual International ACM SIGIR Conference on Research and Development in Information Retrieval, August 15-19, Berkeley, CA (pp. 11-18). New York, NY: ACM.

O'Brien, H., \& Toms, E. G. (2007). Evaluating engagement in interactive search. Paper presented at the Web Information-Seeking and Interaction Workshop, SIGIR Conference, Amsterdam, The Netherlands.

Orlikowski, W. J., \& Yates, J. (1994). Genre repertoire: the structuring of communicative practices in organizations. Administrative Science Quarterly, 39, 541-574.

Orr, R. H. (1970). The scientist as an information processor: a conceptual model illustrated with data on variables related to library utilization. In C. E. Nelson \& D. K. Pollock (Eds.), Communication Among Scientists and Engineers (pp. 143-189). Lexington, MA: Lexington Books.

Paepcke, A. (1996). Information needs in technical work settings and their implications for the design of computer tools. Computer Supported Cooperative Work, 5, 63-92.

Park, S. Y. (1993). The nature of relevance in information retrieval. Library Quarterly, 63(3), 318-351.

Park, T. K. (1994). Toward a theory of user-based relevance: a call for a new paradigm of inquiry. Journal of the American Society for Information Science, 45, 135-141. 
Pejtersen, A. M. (1989). A library system for information retrieval based on a cognitive task analysis and supported by an icon-based interface. In N. J. Belkin \& C. J. van Rijsbergen (Eds.), Proceedings of the 12th International Conference on Research and Development in Information Retrieval, Cambridge, Massachusetts, USA, June 25-28, 1989 (pp. 40-47). New York, NY: ACM.

Petrelli, D., Lanfranchi, V., Moore, P., Ciravegna, F., \& Cadnas, C. (2006). Oh my, where is the end of the context?: dealing with information in a highly complex environment. In I. Ruthven, P. Borlund, P. Ingwersen, N. J. Belkin, A. Tombros \& P. Vakkari (Eds.), Proceedings of the 1st International Symposium on Information Interaction in Context, Copenhagen, DK, October 18-20 (pp. 64-72). New York, NY: ACM.

Pinelli, T. E., Bishop, A. P., Barclay, R. O., \& Kennedy, J. M. (1993). The information-seeking behavior of engineers. Encyclopedia of Library and Information Science, 52(15), 167201.

Pirolli, P. (2007). Information foraging theory: Adaptive interaction with information. Oxford: Oxford Univerity Press.

Ramirez, G., \& de Vries, A. (2006). Relevant contextual features in XML retrieval. In I. Ruthven, P. Borlund, P. Ingwersen, N. J. Belkin, A. Tombros \& P. Vakkari (Eds.), Proceedings of the 1st International Symposium on Information Interaction in Context, Copenhagen, DK, 18-20 October (pp. 95-110). New York, NY: ACM.

Reid, J. (2000). A task-oriented non-interactive evaluation methodology for information retreival systems. Information Retrieval, 2, 115-129.

Robertson, S., Zaragota, H., \& Taylor, M. (2004). Simple BM25 extension to multiple weighted fields. Paper presented at the Proceedings of the thirteenth ACM international conference on Information and knowledge management.

Robertson, S. E., \& Hancock-Banlieu, M. M. (1992). On the evaluation of IR systems. Information Processing and Management, 28(4), 457-466.

Rosso, M. (2005a). Using genre to improve web search (Doctoral dissertation, The University of North Carolina at Chapel Hill): Dissertation Abstracts International 66/03, AAT 3170537.

Rosso, M. (2005b). What type of page is this? Genre as Web descriptor. In M. Marlino, T. Sumner \& F. M. Shipman (Eds.), Proceedings of the ACM/IEEE Joint Conference on Digital Libraries, JCDL 2005, Denver, CO, June 7-11. New York, NY: ACM.

Rosso, M. (2008). User-based identification of Web genres. Journal of the American Society for Information Science \& Technology, 59(7), 1053-1072

Roussinov, D. G., \& Chen, H. (2001). Information navigation on the web by clustering and summarizing query results. Information Processing \& Management, 37, 789-816.

Roussinov, D. G., Crowston, K., Nilan, M., Kwasnik, B., Cai, J., \& Liu, X. (2001). Genre based navigation on the Web. Paper presented at the Hawai'i International Conference on Systems Sciences, Maui, Hawai'i. 
Salton, G. (1989). Automatic text processing: the transformation, analysis, and retrieval of information by computer. Reading, MA: Addison-Wesley.

Salton, G. (1992). The state of retrieval system evaluation. Information Processing \& Management, 28(4), 441-449.

Salton, G. (1996). A new horizon for information science (letter to the editor). Journal of the American Society for Information Science, 47(4), 333.

Santini, M. (2006, August). Common criteria for genre classification: annotation and granularity. Paper presented at the Workshop on Text-based Information Retrieval (TIR06), In Conjunction with ECAI 2006, Riva del Garda, Italy.

Santini, M. (2007, August). Automatic genre identification: towards a flexible classification scheme. Paper presented at the BCS IRSG Symposium: Future Directions in Information Access, Glasgow, Scotland.

Saracevic, T. (1975). Relevance: review of and a framework for the thinking on the notion in information science. Journal of the American Society for Information Science, 26(6), 321-343.

Saracevic, T. (1996). Relevance reconsidered. In P. Ingwersen \& N. O. Pors (Eds.), Information science: Integration in perspective; Proceedings of CoLIS, the 2nd international conference on conceptions of library and information science, Copenhagen, October 1316 (pp. 201-218). Copenhagen: Royal School of Librarianship.

Savage-Knepshield, P. A., \& Belkin, N. J. (1999). Interaction in information retrieval: trends over time. Journal of the American Society for Information Science, 12(50), 1067-1082.

Savolainen, R. (2006). Time as a context of information seeking. Library and Informaiton Science Research, 28, 110-127.

Schamber, L. (1991). User's criteria for evaluation in multimedia information seeking and use situations (Doctoral dissertation, Syracuse University): Dissertation Abstracts International, 52/12, AAT 9214390.

Schamber, L., \& Barry, C. L. (1998). User's criteria for relevance evaluation: a cross-situational comparison. Information Processing \& Management, 34(2/3), 219-236.

Schamber, L., Eisenberg, M. B., \& Nilan, M. S. (1990). A re-examination of relevance: toward a dynamic, situational definition. Information Processing \& Management, 26(6), 755-776.

Serola, S. (2006). City planners' information seeking behavior: Information channels used and information types needed in varying types of perceived work tasks. In I. Ruthven, P. Borlund, P. Ingwersen, N. J. Belkin, A. Tombros \& P. Vakkari (Eds.), Proceedings of the 1st International Symposium on Information Interaction in Context, Copenhagen, Denmark, 18-20 October (pp. 73-79). New York, NY: ACM.

Shen, H., Chen, G., Chen, H., Liu, Y., \& Cheng, X. (2007). Research on enterprise track of TREC 2007. In Proceedings of the Sixteenth Text REtrieval Conference (TREC 2007)

Gaithersburg, MD: NIST. 
Shepherd, M., \& Watters, C. (1999). The functionality attribute of cybergenres. In Proceedings of the 32nd Hawaii International Conference on System Sciences (HICSS '99), January 5-8, 1999, Maui, Hawai: IEEE Computer Society.

Shepherd, M., Watters, C., \& Kennedy, A. (2004). Cybergenre: automatic identification of home pages on the Web. Journal of Web Engineering, 3(3-4), 236-251.

Simon, H. A. (1971). Designing organizations for an information-rich world. In M. Greenberger (Ed.), Computers, communication, and the public interest (pp. 38-52). Batimore, MD: Johns Hopkins Press.

Singer, J., \& Lethbridge, T. C. (1996). Methods for studying maintenance activities. Paper presented at the International Workshop on Empirical Studies of Software Maintenance, Monterey, CA.

Sparck-Jones, K. (2003). Document retrieval: Shallow data, deep theories; historical reflections, potential directions. In F. Sebastiani (Ed.), Advances in information retrieval:

Proceedings of the 25th European Conference on Information Retrieval (ECIR), Pisa, Italy (pp. 1-11). Berlin: Springer.

Sparck-Jones, K. (2006). What's the value of TREC - is there a gap to jump or a chasm to bridge? SIGIR Forum, 40(1), 10-20.

Spink, A., Jansen, B. J., \& Saracevic, T. (2001). Searching the web: the public and their queries. Journal of the American Society for Information Science, 52, 226-234.

Spink, A., Jansen, B. J., Wolfram, D., \& Saracevic, T. (2002). From sex to e-commerce: Web search changes. Computer, 35(3), 107-109.

Spinuzzi, C. (2003). Tracing genres through organizations: a sociocultural approach to information design. Cambridge, MA: MIT Press.

Suchman, L. (1987). Plans and situated actions: the problem of human-machine communication. Cambridge: Cambridge University Press.

Talja, S., Keso, H., \& Pietilainen, T. (1999). The production of 'context' in information seeking research: a metatheoretical view. Information Processing \& Management, 35, 751-763.

Taylor, A. R., Cool, C., Belkin, N. J., \& Amadio, W. J. (2007). Relationships between categories of relevance criteria and stage in task completion. Information Processing \& Management, 43, 1071-1084.

Taylor, R. S. (1968). Question negotiation and information seeking in libraries. College and Research Libraries, 29, 178-194.

Taylor, R. S. (1986). Value added processes in information systems. Westport, CT: Greenwood.

Taylor, R. S. (1991). Information use environments. Progress in Communication Sciences, 10, 217-255.

Tenopir, C., \& King, D. (2004). Communication patterns of engineers. Hoboken, NJ: John Wiley. 
Tiamiyu, M. A. (1992). The relationships between source use and work complexity, decision maker discretion and activity duration in Nigerian government ministries. International Journal of Information Management, 12(2), 130-141.

Tombros, A., Ruthven, I., \& Jose, J. M. (2005). How users assess web pages for information seeking. Journal of the American Society for Information Science and Technology, 56(4), 325-436.

Toms, E. G. (2001). Recognizing digital genre. Bulletin of the American Society for information Science and Technology, 27(2), 20-22.

Toms, E. G., Freund, L., Bartlett, J. C., \& Szigeti, S. (2004, July). Identifying the significant contextual factors of search. Paper presented at the ACM SIGIR Workshop on Information Retrieval in Context, Sheffield, UK.

Toms, E. G., Freund, L., \& Li, C. (2003). WiIRE - the Web interactive information retrieval experimentation system prototype. Information Processing \& Management, 40(4), 655675

Toms, E. G., O'Brien, H., Kopak, R., \& Freund, L. (2005). Searching for relevance in the relevance of search. In F. Crestani \& I. Ruthven (Eds.), Information context: nature, impact, and role: 5th International Conference on Conceptions of Library and Information Science, CoLIS 2005, Glasgow, UK, June 4-8. Berlin: Springer.

Tushman, M. L. (1978). Technical communication in R\&D laboratories: the impact of project work characteristics. Academy of Management Journal, 21, 624-645.

Tyrväinen, P., \& Päivärinta, T. (1999). On rethinking organizational document genres for electronic document management. In Proceedings of the 32nd Hawaii International Conference on System Sciences (HICSS-32), January 5-8, Maui, Hawaii: IEEE Computer Society

Vakkari, P. (2000). Cognition and changes of search terms and tactics during task performance: A longitudinal study. In Proceedings of the RIAO 2000 Conference, 12-14 April, Paris, France (pp. 894-907). Paris: C.I.D.

Vakkari, P. (2001). A theory of the task-based information retrieval process: a summary and generalization of a longitudinal study. Journal of Documentation, 57(1), 44-60.

Vakkari, P. (2003). Task-based information searching. Annual Review of Information Science and Technology, 37, 413-463.

Vakkari, P., \& Hakala, N. (2001). Changes in relevance criteria and problem stages in task performance. Journal of Documentation, 56, 540-562.

Vakkari, P., Savolainen, R., \& Dervin, B. (Eds.). (1997). Information Seeking in Context. London: Taylor Graham.

van Rijsbergen, C. J. (1979). Information retrieval (2nd ed.). London: Butterworths. 
Voorhees, E. M., \& Harman, D. (Eds.). (2005). TREC : experiment and evaluation in information retrieval. Cambridge, MA: MIT Press.

Waddington, P. (1996). Dying for information: an investigation of information overload in the UK and world-wide. London: Reuters Business Information.

Wang, P. (1994). A cognitive model of document selection of real users of IR systems (Doctoral dissertation, University of Maryland College Park): Dissertation Abstracts International, 56/01, AAT 9514595.

Wang, P., Hawk, W. B., \& Tenopir, C. (2000). Users' interaction with World Wide Web resources: an exploratory study using a holistic approach. Information Processing \& Management, 36, 229-251.

Wilson, P. (1973). Situational relevance. Information Processing \& Management, 9, 457-471.

Wilson, T. D. (1984). Cognitive aproach to information-seeking behaviour and information use. Social Science Information Studies, 4(2/3), 197-204.

Wilson, T. D. (1997). Information behaviour: an interdisciplinary perspective. Information Processing and Management, 33(4), 551-572. .

Wilson, T. D. (1999). Exploring models of information behaviour: the 'uncertainty' project. Information Processing and Managemen, 35, 839-849.

Wilson, T. D. (2000). Human information behaviour. Informing Science, 3(2).

Xie, H. (2005). Understanding human-work domain interaction: implications for the design of a corporate digital library. Journal of the American Society for Information Science and Technology, 57(1), 128-143.

Yates, J., \& Orlikowski, W. J. (1992). Genres of organizational communication: a structurational approach to studying communication and media. Academy of Management Review, 17(2), 299-326.

Yates, J., Orlikowski, W. J., \& Okamura, K. (1999). Explicit and implicit structuring of genres in electronic communication: reinforcement and change of social interaction. Organization Science, 10, 83-103.

Yeung, P. C. K., \& Clarke, C. L. A. (2007). A Baysian approach for learning document type relevance. In G. Amati, C. Carpineto \& G. Romano (Eds.), Advances in Information Retrieval, Proceedings of the 29th European Conference on IR Research, ECIR 2007, Rome, Italy, April 2-5 (pp. 753-756). Berlin: Springer

Yeung, P. C. K., Clarke, C. L. A., \& Büttcher, S. (2007). Improving retrieval accuracy by weighting document types with clickthrough data. In Proceedings of the 30th Annual International ACM SIGIR Conference on Research and Development in Information Retrieval, Amsterdam, The Netherlands, July 23-27 (pp. 759-760). New York, NY: ACM. 
Yeung, P. C. K., Freund, L., \& Clarke, C. L. A. (2007). X-Site: a workplace search tool for software engineers. In Proceedings of the 30th Annual International ACM SIGIR Conference on Research and Development in Information Retrieval, Amsterdam, The Netherlands, July 23-27 (pp. 900). New York, NY: ACM.

Yoshioka, T., Herman, G., Yates, J., \& Orlikowski, W. J. (2001). Genre taxonomy: a knowledge repository of communicative actions. ACM Transactions on Information Systems, 19(4), 431-456. 


\section{Appendix 1: Workplace Study Consent Form}

Workplace domain study: Information and Consent Form

Thank you for volunteering to participate in this study, which I am conducting as part of my dissertation research as a doctoral student in the Faculty of Information Studies at the University of Toronto under the supervision of Professors Elaine Toms and Brian Cantwell Smith. This project is supported by ------ and Julie Waterhouse is our Research Associate.

The overall goal of this project is to help software consultants access information more quickly and effectively. Like many employees, software consultants are dependent upon information, but current search systems often fail to provide effective access. This research will focus on developing an approach to information access through a search system which incorporates elements of the work and task environment of software consultants to help target the information search. My goal in this first phase of the project is to better understand the work environment of software consultants. For this purpose, I will interview up to 20 software consultants to identify how you currently find and use information in your work, in order to identify relationships between the tasks you do and the information you need.

Participation in this study is voluntary. It will involve an interview of approximately 1.5 to 2 hours. With your permission, I will also send you a follow-up survey by e-mail asking you to comment on a list of commonly used web-based information resources. You may decline to answer any of the interview questions if you so wish. Further, you may decide to withdraw from this study at any time. The interview will be audio recorded and later transcribed for analysis. The results of this study will be reported without any reference to you specifically. All information that you provide will be treated confidentially and your identity will not be revealed in reporting the study results. No names will be attached to the data files and the files will be deleted after data analysis.

Contact Information:

Luanne Freund

Faculty of Information Studies

Supervisor: Elaine Toms

University of Toronto

Faculty of Information Studies

Freund@fis.utoronto.ca

University of Toronto

Phone: 647-227-9870

toms@fis.utoronto.ca

Signed Consent: I agree to participate in this study conducted by Luanne Freund of the Faculty of Information Studies at the University of Toronto. I have had the opportunity to receive any further details I wanted about the study and I understand that I may withdraw at any time. I wish to take part in the follow-up survey on web-based information resources, and understand that a survey form will be sent to me by e-mail. YES__ NO_ Participant Name (please print):

Participant Signature:

Date: 


\section{Appendix 2: Workplace Study Interview Script}

I'd like to start by telling you a little bit about this project. Our goal is to design an IR device for ---- software consultants, and what I'm interested in at this point is to better understand your work, the role of information in your work, and to try to make the connection between the nature of the work and how information is accessed and used.

IWhen I speak about information, I'm mostly talking about the range of documentation that is available to you - redbooks, product manuals, technical articles, forums etc. but I'm also interested in how you access information through other people. Our project is focusing less on system produced information such as logs and traces.

The way I've structured the interview is to try to work down through the layers of your work - so I have some general questions, and some geared towards specific engagements and some towards specific tasks within engagements, etc.

Do you have any questions before we begin?

\section{Software Engineering}

To start, I'd like to get a general sense of how you use information as a software consultant.

1) How important is it to the job you do?

2) How much time do you spend looking for and using information?

3) Describe your main strategies for finding information.

4) Name your major sources of information - website, databases etc.

5) What are the best specific sources you use - what makes them useful?

6) What are the major problems you encounter when looking for information?

\section{Individual person}

1. What educational and training did you get to do this work?

2. How long have you worked as a Software Consultant?

3. How would you describe your level of expertise within the group of consultants beginner intermediate senior or master?

4. Do you have any areas of specialty as a W----- consultant?

5. Do you have any personal quirks or strategies when it comes to using information? - for example - do you prefer to talk to people or have it written down; do you use Google or prefer internal sources; do you download files and print them, or read online - things like that...

\section{Engagements}

Now, I'd like to talk about information use at the level of individual engagements. To start, I'd like you to tell me what you think of as the main characteristics of an engagement,. For instance, if you had to describe an engagement to another consultant, what characteristics or variables would you mention?

Try to describethreepast engagements you worked on -

1) Describe the engagement, and your role

2) How much time did you spend looking for and using information sources (a little, a lot...)? 
3) Can you describe the questions or problems or issues that motivated you to look for information?

4) Can you describe the main information sources you used?

5) Was there anything about the engagement that made it more difficult or easier to find information?

\section{Work Tasks}

Now, I'd like to look at information use from the point of view of different tasks that are done within engagements.

1. First, I have a list of tasks that we came up with based on some earlier interviews, and I want to get your opinion if you think the list is complete, or if there are some important tasks that are missing. Does it describe the range of work that W----- consultants do?-- Would you add any other important tasks?

Design
Programming
Migration
Security
Testing
Performance Tuning
Installation
Configuration

2. Now, for each one - describe the kinds of information you would be likely to use and where you might find it. Based on your experience, describe how important each task is in the work of software consultants.

\section{Information Tasks}

Now I want to discuss some different types of information searches that you might do in your work.. I'll describe some scenarios, and I want you to tell me for each:

- how common this type of information problem is

- what kind of information you would be likely to use to solve the problem

- where you would go to find the information

1. You've been asked to start a new engagement where you'll have to deal with something you know very little about. So, to prepare, you want to learn about some topic in a general way. 2. You need to do something - design something, install something, or configure, etc. and you need to find out how to do it in terms of the procedures and process.

3. You are in a situation where you have to make a decision about how to proceed with something, and you may have a few options available, so you need information or guidance to support your decision.

4. The customer is asking for some specific information - product specifications, settings, etc. and you need to find this information.

5. You are looking for a tool or application to download - say for testing purposes.

6. You need to find a solution to a problem or system malfunction. 


\section{Problems}

Now we'll try to look at examples of some more specific problems. I want you to look at each of these questions one by one. They are all real questions -mostly about W------application server. I don't want you to try to answer them, but just try to tell me a few things about each one.

- Do you think it could be easy or hard to find an answer - why?

- What type of information task do you think it is - from the list above.

- How might you go about finding information for each?

1. Will IBM HTTP Server 1.3.12.6 work with WebSphere Application Server 3.0.2.4?
2. Should you cache remote references for stateless session beans?
3. Why do I get the message javax.naming.NameNotFoundException: Name jdbc not found in context
"local:". in activity.log on adminstrative and application server startup?
4. Any way to do automatic POST with javascript disabled
Customer has a situation where they must do an automatic HTTP POST.
This can easily be accomplished with javascript, but users of their website
are not required to have javascript enabled.
Does anyone have an alternative?
5. TransactionRollbackException is thrown from the websphere container, but not the nested original
exception in the back stack trace, why?
6. What is the purpose of this tag is and what value it provides? Just to print stuff out? Store messages
into a property file?
<wsNLSEcho key="ISUCCESS" bundle="installapps" message="Installing app successful" />
7. Any integration of Symantec Norton Anti-Virus?
Has anyone done any work with Symantec to integrate it into a WAS application? ...perhaps a content
management application? For instance, if someone submits a file (e.g. a Word doc) to be posted on a
web site, the site owner wants to automatically run an anti-virus program against it before is made
available to other users of the site.
8. Why does an application server not start after it is added to network deployment manager?

\section{Document Characteristics}

Now, I will show you three documents. I would like you to look at each one - you don't need to read it - I'll ask you to tell me about each one:

- What do you think is the general purpose of the document - why did the author write it, and for whom?

- What are the interesting or useful features of the document?

- What might you use such a document for?

\section{Responses to Specific Websites}

That is the end of the interview part, but I would like to ask you to do one more thing for me. I have made up a list of links to some of the key information sites for W-----, and I'd like to send that to you by e-mail, and ask you to fill in some comments for each of the sites - just how often you use the site, and what you impression of the site is - how useful you find it. It shouldn't take very long, and you can do it whenever it's convenient, and return it to me by e-mail.

Now I would like to thank you very much for your help with this project, and for sharing your experience with me. 


\section{Appendix 3: Cross Tabulation of Tasks and Genres}

\begin{tabular}{|c|c|c|c|c|c|c|c|c|}
\hline \multicolumn{2}{|l|}{ GENRES } & Administrate & Architecture & $\begin{array}{l}\text { Capacity } \\
\text { Planning }\end{array}$ & $\begin{array}{c}\text { Competitive } \\
\text { Evaluation }\end{array}$ & Configuration & Debugging & Deployment \\
\hline \multirow{2}{*}{$\begin{array}{l}\text { architecture } \\
\text { (total=545) }\end{array}$} & Count & 39 & 77 & 4 & 1 & 1 & 9 & 3 \\
\hline & Expected Count & 31.2 & 24.7 & 1.9 & 8.1 & 9 & 14.4 & 5.7 \\
\hline \multirow{2}{*}{$\begin{array}{l}\text { collection } \\
\text { (total=218) }\end{array}$} & Count & 8 & 7 & 0 & 3 & 1 & 2 & 0 \\
\hline & Expected Count & 12.5 & 9.9 & 0.8 & 3.2 & 3.6 & 5.8 & 2.3 \\
\hline \multirow{2}{*}{$\begin{array}{l}\text { cookbook } \\
\text { (total=713) }\end{array}$} & Count & 114 & 8 & 0 & 2 & 28 & 13 & 12 \\
\hline & Expected Count & 40.9 & 32.4 & 2.5 & 10.6 & 11.8 & 18.8 & 7.5 \\
\hline \multirow{2}{*}{$\begin{array}{l}\text { demo } \\
\text { (total=339) }\end{array}$} & Count & 14 & 13 & 0 & 1 & 4 & 31 & 5 \\
\hline & Expected Count & 19.4 & 15.4 & 1.2 & 5 & 5.6 & 9 & 3.6 \\
\hline \multirow{2}{*}{$\begin{array}{l}\text { discussion } \\
\text { (total=101) }\end{array}$} & Count & 1 & 1 & 6 & 20 & 0 & 4 & 0 \\
\hline & Expected Count & 5.8 & 4.6 & 0.3 & 1.5 & 1.7 & 2.7 & 1.1 \\
\hline \multirow{2}{*}{$\begin{array}{l}\text { engagement } \\
\text { summary } \\
\text { (total=126) } \\
\end{array}$} & Count & 9 & 0 & 0 & 18 & 0 & 3 & 0 \\
\hline & Expected Count & 7.2 & 5.7 & 0.4 & 1.9 & 2.1 & 3.3 & 1.3 \\
\hline \multirow{2}{*}{$\begin{array}{l}\text { lecture/lab } \\
\text { (total=228) }\end{array}$} & Count & 1 & 0 & 0 & 0 & 0 & 1 & 0 \\
\hline & Expected Count & 13.1 & 10.4 & 0.8 & 3.4 & 3.8 & 6 & 2.4 \\
\hline \multirow{2}{*}{$\begin{array}{l}\text { legal material } \\
\text { (total=19) }\end{array}$} & Count & 2 & 0 & 0 & 0 & 0 & 1 & 0 \\
\hline & Expected Count & 1.1 & 0.9 & 0.1 & 0.3 & 0.3 & 0.5 & 0.2 \\
\hline \multirow{2}{*}{$\begin{array}{l}\text { presentation } \\
(\text { total=1100) }\end{array}$} & Count & 19 & 68 & 0 & 20 & 9 & 14 & 4 \\
\hline & Expected Count & 63.1 & 49.9 & 3.8 & 16.3 & 18.2 & 29.1 & 11.6 \\
\hline \multirow{2}{*}{$\begin{array}{l}\text { product } \\
\text { feedback } \\
\text { (total=95) } \\
\end{array}$} & Count & 12 & 1 & 0 & 0 & 1 & 15 & 2 \\
\hline & Expected Count & 5.4 & 4.3 & 0.3 & 1.4 & 1.6 & 2.5 & 1 \\
\hline \multirow{2}{*}{$\begin{array}{l}\text { reading } \\
\text { material } \\
(\text { total=1629) }\end{array}$} & Count & 56 & 77 & 7 & 20 & 34 & 32 & 23 \\
\hline & Expected Count & 93.4 & 74 & 5.6 & 24.2 & 27 & 43 & 17.2 \\
\hline \multirow{2}{*}{$\begin{array}{l}\text { sales kit } \\
(\text { total }=52)\end{array}$} & Count & 0 & 1 & 0 & 0 & 0 & 0 & 0 \\
\hline & Expected Count & 3 & 2.4 & 0.2 & 0.8 & 0.9 & 1.4 & 0.5 \\
\hline \multirow{2}{*}{$\begin{array}{l}\text { schedule } \\
(\text { total=20) }\end{array}$} & Count & 1 & 0 & 0 & 0 & 0 & 0 & 0 \\
\hline & Expected Count & 1.1 & 0.9 & 0.1 & 0.3 & 0.3 & 0.5 & 0.2 \\
\hline \multirow{2}{*}{$\begin{array}{c}\text { source code } \\
\text { (total=497) }\end{array}$} & Count & 44 & 7 & 1 & 1 & 17 & 22 & 11 \\
\hline & Expected Count & 28.5 & 22.6 & 1.7 & 7.4 & 8.2 & 13.1 & 5.2 \\
\hline \multirow{2}{*}{$\begin{array}{l}\text { tools } \\
\text { (total=19) }\end{array}$} & Count & 3 & 0 & 0 & 0 & 0 & 0 & 0 \\
\hline & Expected Count & 1.1 & 0.9 & 0.1 & 0.3 & 0.3 & 0.5 & 0.2 \\
\hline \multirow{2}{*}{$\begin{array}{l}\text { website/ } \\
\text { repository } \\
\text { (total=82) } \\
\end{array}$} & Count & 9 & 2 & 2 & 0 & 0 & 6 & 0 \\
\hline & Expected Count & 4.7 & 3.7 & 0.3 & 1.2 & 1.4 & 2.2 & 0.9 \\
\hline \multirow{2}{*}{$\begin{array}{l}\text { white paper } \\
\text { (total=9) }\end{array}$} & Count & 0 & 1 & 0 & 0 & 1 & 0 & 1 \\
\hline & Expected Count & 0.5 & 0.4 & 0 & 0.1 & 0.1 & 0.2 & 0.1 \\
\hline \multirow[t]{2}{*}{ TOTAL } & Count & 332 & 263 & 20 & 86 & 96 & 153 & 61 \\
\hline & $\%$ of Total & $5.70 \%$ & $4.50 \%$ & $0.30 \%$ & $1.50 \%$ & $1.70 \%$ & $2.60 \%$ & $1.10 \%$ \\
\hline
\end{tabular}




\begin{tabular}{|c|c|c|c|c|c|c|c|c|c|}
\hline \multicolumn{2}{|l|}{ GENRES } & $\begin{array}{l}\text { Proof of } \\
\text { Concept }\end{array}$ & $\begin{array}{c}\text { Product } \\
\text { Presentation }\end{array}$ & $\begin{array}{l}\text { Project } \\
\text { Manag't }\end{array}$ & $\begin{array}{l}\text { Product } \\
\text { Review }\end{array}$ & Security & Test & Compare & Contacts \\
\hline \multirow[t]{2}{*}{ Architecture } & Count & 11 & 0 & 7 & 3 & 12 & 0 & 11 & 0 \\
\hline & Expected Count & 4.6 & 2 & 4.8 & 4 & 5.8 & 5.4 & 4.2 & 0.4 \\
\hline \multirow[t]{2}{*}{ collection } & Count & 1 & 0 & 0 & 11 & 2 & 1 & 3 & 2 \\
\hline & Expected Count & 1.8 & 0.8 & 1.9 & 1.6 & 2.3 & 2.1 & 1.7 & 0.2 \\
\hline \multirow[t]{2}{*}{ cookbook } & Count & 9 & 0 & 11 & 6 & 14 & 3 & 4 & 0 \\
\hline & Expected Count & 6 & 2.6 & 6.3 & 5.3 & 7.6 & 7 & 5.5 & 0.5 \\
\hline \multirow[t]{2}{*}{ demo } & Count & 1 & 0 & 0 & 0 & 3 & 15 & 0 & 0 \\
\hline & Expected Count & 2.9 & 1.2 & 3 & 2.5 & 3.6 & 3.3 & 2.6 & 0.2 \\
\hline \multirow[t]{2}{*}{ discussion } & Count & 0 & 0 & 0 & 0 & 0 & 0 & 2 & 0 \\
\hline & Expected Count & 0.9 & 0.4 & 0.9 & 0.7 & 1.1 & 1 & 0.8 & 0.1 \\
\hline \multirow[t]{2}{*}{$\begin{array}{l}\text { engagement } \\
\text { summary }\end{array}$} & Count & 11 & 0 & 17 & 2 & 0 & 0 & 0 & 0 \\
\hline & Expected Count & 1.1 & 0.5 & 1.1 & 0.9 & 1.3 & 1.2 & 1 & 0.1 \\
\hline \multirow[t]{2}{*}{ lecture/lab } & Count & 1 & 0 & 0 & 0 & 0 & 0 & 0 & 0 \\
\hline & Expected Count & 1.9 & 0.8 & 2 & 1.7 & 2.4 & 2.2 & 1.8 & 0.2 \\
\hline \multirow[t]{2}{*}{ legal material } & Count & 0 & 0 & 0 & 0 & 0 & 0 & 0 & 0 \\
\hline & Expected Count & 0.2 & 0.1 & 0.2 & 0.1 & 0.2 & 0.2 & 0.1 & 0 \\
\hline \multirow[t]{2}{*}{ presentation } & Count & 1 & 18 & 3 & 2 & 8 & 12 & 4 & 1 \\
\hline & Expected Count & 9.3 & 4 & 9.7 & 8.2 & 11.8 & 10.8 & 8.5 & 0.8 \\
\hline \multirow[t]{2}{*}{$\begin{array}{l}\text { product } \\
\text { feedback }\end{array}$} & Count & 0 & 1 & 0 & 1 & 0 & 2 & 2 & 0 \\
\hline & Expected Count & 0.8 & 0.3 & 0.8 & 0.7 & 1 & 0.9 & 0.7 & 0.1 \\
\hline \multirow[t]{2}{*}{$\begin{array}{l}\text { reading } \\
\text { material }\end{array}$} & Count & 8 & 2 & 3 & 11 & 11 & 15 & 13 & 0 \\
\hline & Expected Count & 13.8 & 5.9 & 14.3 & 12.1 & 17.4 & 16 & 12.7 & 1.1 \\
\hline \multirow[t]{2}{*}{ sales kit } & Count & 0 & 0 & 2 & 0 & 0 & 0 & 1 & 0 \\
\hline & Expected Count & 0.4 & 0.2 & 0.5 & 0.4 & 0.6 & 0.5 & 0.4 & 0 \\
\hline \multirow[t]{2}{*}{ schedule } & Count & 0 & 0 & 5 & 0 & 0 & 0 & 3 & 0 \\
\hline & Expected Count & 0.2 & 0.1 & 0.2 & 0.1 & 0.2 & 0.2 & 0.2 & 0 \\
\hline \multirow[t]{2}{*}{ source code } & Count & 6 & 0 & 0 & 6 & 12 & 9 & 2 & 0 \\
\hline & Expected Count & 4.2 & 1.8 & 4.4 & 3.7 & 5.3 & 4.9 & 3.9 & 0.3 \\
\hline \multirow[t]{2}{*}{ tools } & Count & 0 & 0 & 3 & 0 & 0 & 0 & 0 & 0 \\
\hline & Expected Count & 0.2 & 0.1 & 0.2 & 0.1 & 0.2 & 0.2 & 0.1 & 0 \\
\hline \multirow[t]{2}{*}{$\begin{array}{l}\text { website/ } \\
\text { repository }\end{array}$} & Count & 0 & 0 & 0 & 1 & 0 & 0 & 0 & 1 \\
\hline & Expected Count & 0.7 & 0.3 & 0.7 & 0.6 & 0.9 & 0.8 & 0.6 & 0.1 \\
\hline \multirow[t]{2}{*}{ white paper } & Count & 0 & 0 & 0 & 0 & 0 & 0 & 0 & 0 \\
\hline & Expected Count & 0.1 & 0 & 0.1 & 0.1 & 0.1 & 0.1 & 0.1 & 0 \\
\hline \multirow[t]{2}{*}{ TOTAL } & Count & 49 & 21 & 51 & 43 & 62 & 57 & 45 & 4 \\
\hline & $\%$ of Total & $0.80 \%$ & $0.40 \%$ & $0.90 \%$ & $0.70 \%$ & $1.10 \%$ & $1.00 \%$ & $0.80 \%$ & $0.10 \%$ \\
\hline
\end{tabular}




\begin{tabular}{|c|c|c|c|c|c|c|c|c|}
\hline \multicolumn{2}{|l|}{ GENRES } & Development & $\begin{array}{l}\text { Discovery } \\
\text { Session }\end{array}$ & Implementation & Installation & Integration & Migration & $\begin{array}{c}\text { Performance } \\
\text { Tuning }\end{array}$ \\
\hline \multirow[t]{2}{*}{ Architecture } & Count & 17 & 2 & 12 & 2 & 43 & 14 & 23 \\
\hline & Expected Count & 13.6 & 3.9 & 11.8 & 12.6 & 20.3 & 16.2 & 15.4 \\
\hline \multirow[t]{2}{*}{ collection } & Count & 6 & 1 & 2 & 1 & 3 & 7 & 6 \\
\hline & Expected Count & 5.5 & 1.5 & 4.7 & 5 & 8.1 & 6.5 & 6.2 \\
\hline \multirow[t]{2}{*}{ cookbook } & Count & 19 & 5 & 10 & 51 & 38 & 30 & 18 \\
\hline & Expected Count & 17.8 & 5 & 15.4 & 16.5 & 26.6 & 21.2 & 20.2 \\
\hline \multirow[t]{2}{*}{ demo } & Count & 14 & 0 & 3 & 20 & 15 & 4 & 21 \\
\hline & Expected Count & 8.5 & 2.4 & 7.3 & 7.8 & 12.6 & 10.1 & 9.6 \\
\hline \multirow[t]{2}{*}{ discussion } & Count & 2 & 0 & 0 & 0 & 1 & 1 & 8 \\
\hline & Expected Count & 2.5 & 0.7 & 2.2 & 2.3 & 3.8 & 3 & 2.9 \\
\hline \multirow[t]{2}{*}{$\begin{array}{l}\text { engagement } \\
\text { summary }\end{array}$} & Count & 0 & 11 & 1 & 0 & 2 & 10 & 5 \\
\hline & Expected Count & 3.2 & 0.9 & 2.7 & 2.9 & 4.7 & 3.7 & 3.6 \\
\hline \multirow[t]{2}{*}{ lecture/lab } & Count & 4 & 0 & 2 & 0 & 0 & 0 & 1 \\
\hline & Expected Count & 5.7 & 1.6 & 4.9 & 5.3 & 8.5 & 6.8 & 6.5 \\
\hline \multirow[t]{2}{*}{$\begin{array}{l}\text { legal } \\
\text { material }\end{array}$} & Count & 0 & 1 & 0 & 0 & 0 & 1 & 0 \\
\hline & Expected Count & 0.5 & 0.1 & 0.4 & 0.4 & 0.7 & 0.6 & 0.5 \\
\hline \multirow[t]{2}{*}{ presentation } & Count & 20 & 5 & 2 & 4 & 8 & 31 & 23 \\
\hline & Expected Count & 27.5 & 7.8 & 23.7 & 25.4 & 41 & 32.7 & 31.1 \\
\hline \multirow[t]{2}{*}{$\begin{array}{l}\text { product } \\
\text { feedback }\end{array}$} & Count & 1 & 2 & 0 & 2 & 2 & 5 & 2 \\
\hline & Expected Count & 2.4 & 0.7 & 2.1 & 2.2 & 3.5 & 2.8 & 2.7 \\
\hline \multirow[t]{2}{*}{$\begin{array}{l}\text { reading } \\
\text { material }\end{array}$} & Count & 35 & 10 & 77 & 41 & 50 & 47 & 44 \\
\hline & Expected Count & 40.8 & 11.5 & 35.2 & 37.7 & 60.8 & 48.4 & 46.1 \\
\hline \multirow[t]{2}{*}{ sales kit } & Count & 0 & 1 & 0 & 0 & 0 & 4 & 0 \\
\hline & Expected Count & 1.3 & 0.4 & 1.1 & 1.2 & 1.9 & 1.5 & 1.5 \\
\hline \multirow[t]{2}{*}{ schedule } & Count & 0 & 0 & 0 & 0 & 0 & 1 & 0 \\
\hline & Expected Count & 0.5 & 0.1 & 0.4 & 0.5 & 0.7 & 0.6 & 0.6 \\
\hline \multirow[t]{2}{*}{ source code } & Count & 26 & 0 & 16 & 13 & 48 & 15 & 8 \\
\hline & Expected Count & 12.4 & 3.5 & 10.7 & 11.5 & 18.5 & 14.8 & 14.1 \\
\hline \multirow[t]{2}{*}{ tools } & Count & 0 & 3 & 0 & 0 & 3 & 2 & 0 \\
\hline & Expected Count & 0.5 & 0.1 & 0.4 & 0.4 & 0.7 & 0.6 & 0.5 \\
\hline \multirow[t]{2}{*}{$\begin{array}{l}\text { website/ } \\
\text { repository }\end{array}$} & Count & 1 & 0 & 0 & 0 & 3 & 0 & 5 \\
\hline & Expected Count & 2.1 & 0.6 & 1.8 & 1.9 & 3.1 & 2.4 & 2.3 \\
\hline \multirow[t]{2}{*}{ white paper } & Count & 0 & 0 & 0 & 0 & 0 & 0 & 0 \\
\hline & Expected Count & 0.2 & 0.1 & 0.2 & 0.2 & 0.3 & 0.3 & 0.3 \\
\hline \multirow[t]{2}{*}{ TOTAL } & Count & 145 & 41 & 125 & 134 & 216 & 172 & 164 \\
\hline & $\%$ of Total & $2.50 \%$ & $0.70 \%$ & $2.20 \%$ & $2.30 \%$ & $3.70 \%$ & $3.00 \%$ & $2.80 \%$ \\
\hline
\end{tabular}




\begin{tabular}{|c|c|c|c|c|c|c|c|c|}
\hline \multicolumn{2}{|l|}{ GENRES } & Demonstrate & Document & Educate & Example & Guide & Index & Market \\
\hline \multirow[t]{2}{*}{ Architecture } & Count & 49 & 58 & 107 & 16 & 0 & 0 & 1 \\
\hline & Expected Count & 45.5 & 39.5 & 179.1 & 13.1 & 0.8 & 1.1 & 8.8 \\
\hline \multirow[t]{2}{*}{ collection } & Count & 15 & 26 & 81 & 2 & 1 & 11 & 1 \\
\hline & Expected Count & 18.2 & 15.8 & 71.6 & 5.2 & 0.3 & 0.5 & 3.5 \\
\hline \multirow[t]{2}{*}{ cookbook } & Count & 90 & 54 & 126 & 22 & 0 & 0 & 6 \\
\hline & Expected Count & 59.6 & 51.7 & 234.3 & 17.1 & 1.1 & 1.5 & 11.4 \\
\hline \multirow[t]{2}{*}{ demo } & Count & 99 & 4 & 48 & 12 & 0 & 0 & 1 \\
\hline & Expected Count & 28.3 & 24.6 & 111.4 & 8.1 & 0.5 & 0.7 & 5.4 \\
\hline \multirow[t]{2}{*}{ discussion } & Count & 12 & 17 & 21 & 0 & 0 & 0 & 0 \\
\hline & Expected Count & 8.4 & 7.3 & 33.2 & 2.4 & 0.2 & 0.2 & 1.6 \\
\hline \multirow[t]{2}{*}{$\begin{array}{l}\text { engagement } \\
\text { summary }\end{array}$} & Count & 6 & 8 & 11 & 3 & 0 & 0 & 5 \\
\hline & Expected Count & 10.5 & 9.1 & 41.4 & 3 & 0.2 & 0.3 & 2 \\
\hline \multirow[t]{2}{*}{ lecture/lab } & Count & 17 & 1 & 183 & 13 & 0 & 0 & 3 \\
\hline & Expected Count & 19.1 & 16.5 & 74.9 & 5.5 & 0.4 & 0.5 & 3.7 \\
\hline \multirow[t]{2}{*}{ legal material } & Count & 2 & 4 & 4 & 0 & 0 & 0 & 2 \\
\hline & Expected Count & 1.6 & 1.4 & 6.2 & 0.5 & 0 & 0 & 0.3 \\
\hline \multirow[t]{2}{*}{ presentation } & Count & 54 & 52 & 603 & 3 & 0 & 0 & 26 \\
\hline & Expected Count & 91.9 & 79.8 & 361.4 & 26.4 & 1.7 & 2.3 & 17.7 \\
\hline \multirow[t]{2}{*}{$\begin{array}{l}\text { product } \\
\text { feedback }\end{array}$} & Count & 2 & 16 & 18 & 0 & 0 & 0 & 1 \\
\hline & Expected Count & 7.9 & 6.9 & 31.2 & 2.3 & 0.1 & 0.2 & 1.5 \\
\hline \multirow[t]{2}{*}{$\begin{array}{l}\text { reading } \\
\text { material }\end{array}$} & Count & 74 & 144 & 592 & 39 & 6 & 0 & 14 \\
\hline & Expected Count & 136.1 & 118.1 & 535.2 & 39.1 & 2.5 & 3.4 & 26.2 \\
\hline \multirow[t]{2}{*}{ sales kit } & Count & 1 & 4 & 4 & 0 & 0 & 0 & 32 \\
\hline & Expected Count & 4.3 & 3.8 & 17.1 & 1.2 & 0.1 & 0.1 & 0.8 \\
\hline \multirow[t]{2}{*}{ schedule } & Count & 0 & 2 & 4 & 0 & 0 & 0 & 0 \\
\hline & Expected Count & 1.7 & 1.5 & 6.6 & 0.5 & 0 & 0 & 0.3 \\
\hline \multirow[t]{2}{*}{ source code } & Count & 56 & 24 & 61 & 29 & 2 & 0 & 1 \\
\hline & Expected Count & 41.5 & 36 & 163.3 & 11.9 & 0.8 & 1 & 8 \\
\hline \multirow[t]{2}{*}{ tools } & Count & 2 & 0 & 3 & 0 & 0 & 0 & 0 \\
\hline & Expected Count & 1.6 & 1.4 & 6.2 & 0.5 & 0 & 0 & 0.3 \\
\hline \multirow[t]{2}{*}{$\begin{array}{l}\text { website/ } \\
\text { repository }\end{array}$} & Count & 5 & 5 & 35 & 0 & 0 & 1 & 0 \\
\hline & Expected Count & 6.9 & 5.9 & 26.9 & 2 & 0.1 & 0.2 & 1.3 \\
\hline \multirow[t]{2}{*}{ white paper } & Count & 0 & 1 & 2 & 0 & 0 & 0 & 0 \\
\hline & Expected Count & 0.8 & 0.7 & 3 & 0.2 & 0 & 0 & 0.1 \\
\hline \multirow[t]{2}{*}{ TOTAL } & Count & 484 & 420 & 1903 & 139 & 9 & 12 & 93 \\
\hline & $\%$ of Total & $8.40 \%$ & $7.30 \%$ & $32.90 \%$ & $2.40 \%$ & $0.20 \%$ & $0.20 \%$ & $1.60 \%$ \\
\hline
\end{tabular}




\begin{tabular}{|c|c|c|c|c|c|c|c|c|}
\hline \multicolumn{2}{|l|}{ GENRES } & Method & Reference & Roadmap & Standards & Support & $\begin{array}{c}\text { Technical } \\
\text { Info }\end{array}$ & Tool \\
\hline \multirow[t]{2}{*}{ Architecture } & Count & 3 & 10 & 1 & 4 & 5 & 0 & 0 \\
\hline & Expected Count & 3.6 & 6.6 & 0.5 & 1.2 & 6.7 & 10.8 & 7.5 \\
\hline \multirow[t]{2}{*}{ collection } & Count & 0 & 8 & 1 & 0 & 2 & 3 & 0 \\
\hline & Expected Count & 1.4 & 2.6 & 0.2 & 0.5 & 2.7 & 4.3 & 3 \\
\hline \multirow[t]{2}{*}{ cookbook } & Count & 1 & 7 & 1 & 0 & 8 & 2 & 1 \\
\hline & Expected Count & 4.7 & 8.6 & 0.6 & 1.6 & 8.7 & 14.2 & 9.8 \\
\hline \multirow[t]{2}{*}{ demo } & Count & 3 & 0 & 0 & 0 & 3 & 1 & 4 \\
\hline & Expected Count & 2.2 & 4.1 & 0.3 & 0.8 & 4.2 & 6.7 & 4.7 \\
\hline \multirow[t]{2}{*}{ discussion } & Count & 0 & 1 & 0 & 0 & 3 & 0 & 1 \\
\hline & Expected Count & 0.7 & 1.2 & 0.1 & 0.2 & 1.2 & 2 & 1.4 \\
\hline \multirow[t]{2}{*}{$\begin{array}{l}\text { engagement } \\
\text { summary }\end{array}$} & Count & 2 & 1 & 0 & 1 & 0 & 0 & 0 \\
\hline & Expected Count & 0.8 & 1.5 & 0.1 & 0.3 & 1.5 & 2.5 & 1.7 \\
\hline \multirow[t]{2}{*}{ lecture/lab } & Count & 0 & 0 & 0 & 0 & 1 & 0 & 0 \\
\hline & Expected Count & 1.5 & 2.8 & 0.2 & 0.5 & 2.8 & 4.5 & 3.1 \\
\hline \multirow[t]{2}{*}{ legal material } & Count & 0 & 2 & 0 & 0 & 0 & 0 & 0 \\
\hline & Expected Count & 0.1 & 0.2 & 0 & 0 & 0.2 & 0.4 & 0.3 \\
\hline \multirow[t]{2}{*}{ presentation } & Count & 11 & 6 & 0 & 1 & 5 & 58 & 5 \\
\hline & Expected Count & 7.2 & 13.3 & 0.9 & 2.5 & 13.5 & 21.8 & 15.2 \\
\hline \multirow[t]{2}{*}{ product feedback } & Count & 0 & 2 & 0 & 0 & 3 & 2 & 0 \\
\hline & Expected Count & 0.6 & 1.1 & 0.1 & 0.2 & 1.2 & 1.9 & 1.3 \\
\hline \multirow[t]{2}{*}{ reading material } & Count & 18 & 19 & 2 & 7 & 37 & 46 & 15 \\
\hline & Expected Count & 10.7 & 19.7 & 1.4 & 3.7 & 20 & 32.3 & 22.5 \\
\hline \multirow[t]{2}{*}{ sales kit } & Count & 0 & 1 & 0 & 0 & 0 & 1 & 0 \\
\hline & Expected Count & 0.3 & 0.6 & 0 & 0.1 & 0.6 & 1 & 0.7 \\
\hline \multirow[t]{2}{*}{ schedule } & Count & 0 & 4 & 0 & 0 & 0 & 0 & 0 \\
\hline & Expected Count & 0.1 & 0.2 & 0 & 0 & 0.2 & 0.4 & 0.3 \\
\hline \multirow[t]{2}{*}{ source code } & Count & 0 & 6 & 0 & 0 & 1 & 0 & 53 \\
\hline & Expected Count & 3.3 & 6 & 0.4 & 1.1 & 6.1 & 9.9 & 6.9 \\
\hline \multirow[t]{2}{*}{ tools } & Count & 0 & 0 & 0 & 0 & 0 & 0 & 0 \\
\hline & Expected Count & 0.1 & 0.2 & 0 & 0 & 0.2 & 0.4 & 0.3 \\
\hline \multirow[t]{2}{*}{$\begin{array}{l}\text { website/ } \\
\text { repository }\end{array}$} & Count & 0 & 3 & 0 & 0 & 3 & 0 & 0 \\
\hline & Expected Count & 0.5 & 1 & 0.1 & 0.2 & 1 & 1.6 & 1.1 \\
\hline \multirow[t]{2}{*}{ white paper } & Count & 0 & 0 & 0 & 0 & 0 & 2 & 1 \\
\hline & Expected Count & 0.1 & 0.1 & 0 & 0 & 0.1 & 0.2 & 0.1 \\
\hline \multirow[t]{2}{*}{ TOTAL } & Count & 38 & 70 & 5 & 13 & 71 & 115 & 80 \\
\hline & $\%$ of Total & $0.70 \%$ & $1.20 \%$ & $0.10 \%$ & $0.20 \%$ & $1.20 \%$ & $2.00 \%$ & $1.40 \%$ \\
\hline
\end{tabular}




\section{Appendix 4: Validation Survey E-Mail Advertisement}

Team,

You can help to improve cross-site search capabilities for Software Services by participating in a concise 30-minute web-based survey.

Fifteen of our colleagues have already provided input to Phase 1 of a study by the ----- last year. The research team used the results of those detailed interviews, and now has a model to validate in Phase 2. The data collected from the web-based survey of software consultants will be used to provide this validation. The model considers several dimensions of "why you are searching," to improve the relevance of the search results.

The survey will take you about 30 minutes, and can be completed online at https://--SSurvey/index.jsp anytime between Friday February 25 and March 7, 2005. A short briefing on the project will be provided by conference call on Tuesday March 1 at 1:00 EST (slides attached). This call will be recorded and archived for later viewing.

Luanne Freund is the lead for this research project. She can be reached for questions and comments at -------. More information and some of the results from Phase 1 are available for your information, at http://www.fis.utoronto.ca/phd/freund/sssearch.htm

Thanks for joining in! 


\section{Appendix 5: Validation Survey Instruments}

\section{Survey on Work Activities and Information Use: Software Services Group}

\section{Sponsored by}

The overall goal of this project is to help Software Services consultants access information more quickly and effectively by designing a search system that can provide the right information for the job . We are studying the relationships between different work activities and different types of information, so that we can model this and use it to improve search results. Your help with this questionnaire will allow us to validate our model with your work experience, and will contribute directly to the design of the new system.

The questionnaire to follow has 4 parts, and should take about 30 minutes to complete. We ask for some brief background information on participants, and then ask a series of questions relating to: the information sources you typically use, the nature of your work activities, and the different types of information you use in various situations.

We ask that you base your responses on your own experience and judgement - there are no "right" answers.

\section{Please note that:}

This research project is sponsored by --- which has approved the use of work time to complete this survey;

- Your email address will be stored separately from the rest of the data collected, and will not be passed on or used to contact you;

- your identity will not be revealed in the reporting of the study results;

- IBM project coordinators and team managers will not have access to individual participant results;

- Your participation is voluntary - you may withdraw at any time, and you may decline to answer any question.

- If you have any questions about this study, please send your request to lfreund@ca.ibm.com.

- Thank-you for your interest and participation!

\section{Top of Form}

\section{If you are ready to participate in this study, enter:}

\section{Email address:}




\section{Section 1: Background Information}

1. Did you provide input to Phase I of this project, either through an interview or a focus group?

$$
\text { No } \mathbb{C} \text { Yes } \mathbb{C}
$$

2. Have you seen a presentation of the results from Phase I of this project ? No $\mathbb{C}$ Yes $\square$

3. Job Title

$\begin{array}{lll}\text { 4. Job Band } & -\quad \text { (optional) } & \text { 5. Years of experience in current work }\end{array}$

6. Software Brand Websphere
7. Education

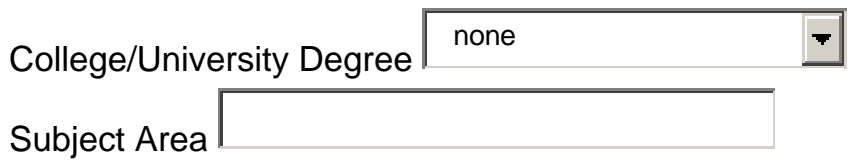
8. Age $\longdiv { 2 1 - 2 5 \quad \square }$
7. Gender: female $\mathbf{C}$ male $\mathbf{C}$

\section{Section 2: Searching for Information}

In the course of working, how often do you use the following sources to find information? (Please select a number from one to 5).

never

very frequently

\begin{tabular}{|c|c|c|c|c|c|}
\hline & 1 & 2 & 3 & 4 & 5 \\
\hline internet search engines & $\mathbf{C}$ & E & $\mathbf{E}$ & $\mathbf{E}$ & $\mathbf{C}$ \\
\hline product documentation & $\mathbf{C}$ & C & $\mathbf{C}$ & $\mathbf{C}$ & $\mathbf{C}$ \\
\hline Redbooks & $\mathbf{C}$ & $\mathbb{E}$ & $\mathbf{C}$ & $\mathbf{C}$ & C \\
\hline bookmarks / favorites & $\mathbf{E}$ & $\mathbb{E}$ & $\mathbf{E}$ & $\mathbf{C}$ & $\mathbf{E}$ \\
\hline newsgroups & C & E & $\mathbf{C}$ & C & C \\
\hline teamrooms & $\mathbf{C}$ & $\mathbf{C}$ & $\mathbf{C}$ & $\mathbf{C}$ & $\mathbf{C}$ \\
\hline Intellectual Capital Repositories & $\mathbf{C}$ & C & $\mathbf{C}$ & $\mathbf{C}$ & $\mathbf{C}$ \\
\hline other internal databases & $\mathbf{C}$ & $\mathbb{E}$ & $\mathbf{C}$ & $\mathbf{C}$ & $\mathbf{C}$ \\
\hline ---.com site search engine & $\mathbf{C}$ & $\mathbb{E}$ & $\mathbf{C}$ & $\mathbf{C}$ & $\mathbf{C}$ \\
\hline --- support website & $\mathbf{E}$ & $\mathbf{E}$ & $\mathbf{C}$ & $\mathbf{C}$ & $\mathbf{C}$ \\
\hline --- intranet (w3) search engine & $\mathbf{C}$ & E & $\mathbf{C}$ & $\mathbf{C}$ & C \\
\hline
\end{tabular}




\begin{tabular}{|l|c|c|c|c|c|}
\hline $\begin{array}{l}\text { Developers Domain } \\
\begin{array}{l}\text { personal websites (incl. your } \\
\text { own) }\end{array}\end{array}$ & $\mathbf{C}$ & $\mathbf{C}$ & $\mathbf{C}$ & $\mathbf{C}$ & $\mathbf{C}$ \\
\hline \hline \begin{tabular}{l} 
PMR/APAR databases \\
\hline your own document collection
\end{tabular} & $\mathbf{C}$ & $\mathbf{C}$ & $\mathbf{C}$ & $\mathbf{C}$ & $\mathbf{C}$ \\
\hline \hline other: & $\mathbf{C}$ & $\mathbf{C}$ & $\mathbf{C}$ & $\mathbf{C}$ & $\mathbf{C}$ \\
\hline \hline other: & $\mathbf{C}$ & $\mathbf{C}$ & $\mathbf{C}$ & $\mathbf{C}$ & $\mathbf{C}$ \\
\hline \hline
\end{tabular}

\section{Section 3: Describing Software Services Work Activities}

There are 11 software services activities listed below with brief definitions and some statements relating to each activity. For each one, indicate the degree to which you agree or disagree with the statements, by selecting a number from 1 to 5 . Base your answers on your own judgement and experience, within your specialization.

(Note: this table was repeated for each of the 11 work tasks.)

1. Architecture - determining the components of a computer system and the way they interact with one another

disagree strongly

agree strongly

\begin{tabular}{|l|c|c|c|c|c|}
\hline & 1 & 2 & 3 & 4 & 5 \\
\hline is a high level activity & C & C & C & C & C \\
\hline is a technology-centric activity & C & C & C & C & C \\
\hline is a product-centric activity & C & C & C & C & C \\
\hline $\begin{array}{l}\text { involves a lot of interaction with } \\
\text { customers }\end{array}$ & C & C & C & C & C \\
\hline $\begin{array}{l}\text { requires on the job experience to do } \\
\text { well }\end{array}$ & C & C & C & C & C \\
\hline $\begin{array}{l}\text { requires in-depth knowledge to do } \\
\text { well }\end{array}$ & C & C & C & C & C \\
\hline is a complex activity & C & C & C & C & C \\
\hline can employ standard methods & C & C & C & C & C \\
\hline Comments: & & & & \\
\hline
\end{tabular}




\section{Section 4: Use Scenarios and Information Types}

In this section we ask you to identify how useful different types of information are for different intended uses. For each scenario, based on your experience, rate each type of information on a scale of 1-5 to indicate likely usefulness.

(Note: this table was repeated for each of the five information tasks.)

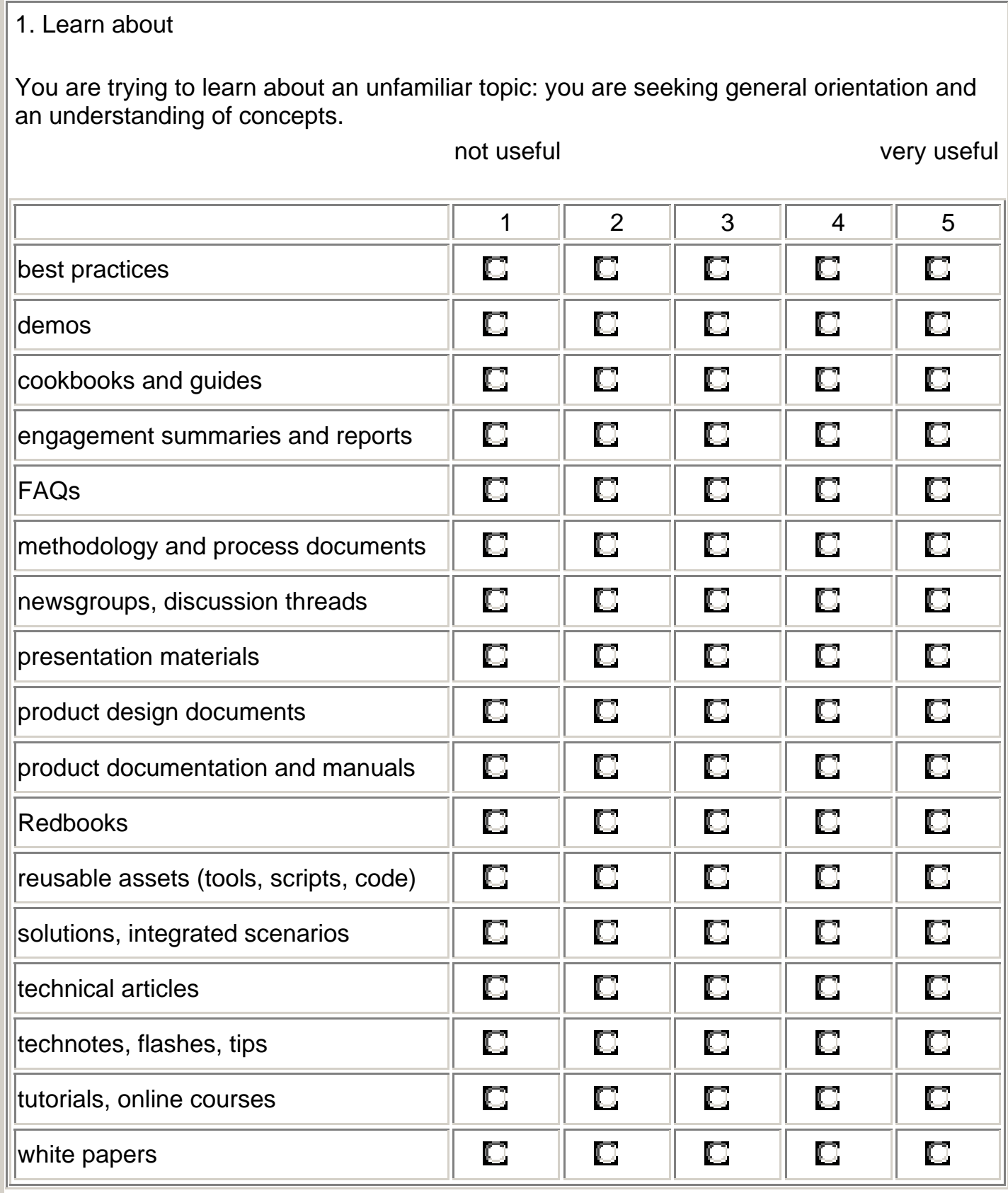




\section{Final Page}

That's all Folks!

Thank you for participating in this study. We will be posting updates and reports on the project on the project webpage.

As a small token of thanks, and in support of our local Toronto hockey team, we are giving away a Toronto Maple Leafs hockey jersey to one lucky participant.

If you would like to be in on the draw, please enter your email address here:

Email Address

For more information or questions, please feel free to contact one of us:

Luanne Freund

Doctoral Candidate

Faculty of Information Studies

University of Toronto

lfreund@ca.ibm.com

Elaine Toms

Associate Professor

Faculty of Management

Dalhousie University, Halifax

etoms@dal.ca

Julie Waterhouse 


\section{Appendix 6: Correlations among Work Task Characteristics*}

\begin{tabular}{|c|c|c|c|c|c|c|c|c|c|}
\hline & & 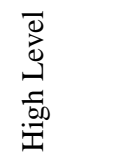 & 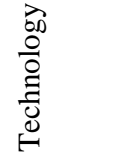 & 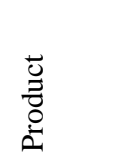 & 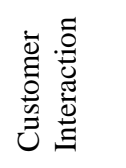 & 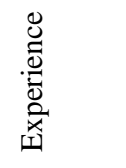 & 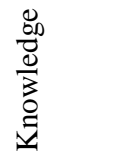 & & 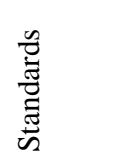 \\
\hline \multirow[t]{3}{*}{ High Level } & $\begin{array}{l}\text { Correlation } \\
\text { Coefficient }\end{array}$ & 1.000 & $-.292(* *)$ & $-.364(* *)$ & $.318(* *)$ & -.019 & -.136 & -.028 & $.194(* *)$ \\
\hline & Sig. (2-tailed) & . & .000 & .000 & .000 & .779 & .086 & .686 & .004 \\
\hline & $\mathrm{N}$ & 217 & 216 & 215 & 216 & 215 & 161 & 217 & 217 \\
\hline \multirow[t]{3}{*}{ Technology } & $\begin{array}{l}\text { Correlation } \\
\text { Coefficient }\end{array}$ & $-.292(* *)$ & 1.000 & $.645(* *)$ & $-.145(*)$ & .133 & $.210(* *)$ & $.142\left(^{*}\right)$ & .133 \\
\hline & Sig. (2-tailed) & .000 & . & .000 & .033 & .051 & .008 & .036 & .051 \\
\hline & $\mathrm{N}$ & 216 & 216 & 214 & 215 & 214 & 160 & 216 & 216 \\
\hline \multirow[t]{3}{*}{ Product } & $\begin{array}{l}\text { Correlation } \\
\text { Coefficient }\end{array}$ & $-.364(* *)$ & $.645(* *)$ & 1.000 & $-.136\left(^{*}\right)$ & -.009 & $.173(*)$ & .059 & .011 \\
\hline & Sig. (2-tailed) & .000 & .000 & . & .047 & .900 & .030 & .388 & .873 \\
\hline & $\mathrm{N}$ & 215 & 214 & 215 & 215 & 213 & 159 & 215 & 215 \\
\hline \multirow[t]{3}{*}{$\begin{array}{l}\text { Customer } \\
\text { Interaction }\end{array}$} & $\begin{array}{l}\text { Correlation } \\
\text { Coefficient }\end{array}$ & $.318(* *)$ & $-.145\left(^{*}\right)$ & $-.136(*)$ & 1.000 & $.160\left(^{*}\right)$ & .059 & .071 & $.205(* *)$ \\
\hline & Sig. (2-tailed) & .000 & .033 & .047 & $\cdot$ & .019 & .460 & .301 & .002 \\
\hline & $\mathrm{N}$ & 216 & 215 & 215 & 216 & 214 & 160 & 216 & 216 \\
\hline \multirow[t]{3}{*}{ Experience } & $\begin{array}{l}\text { Correlation } \\
\text { Coefficient }\end{array}$ & -.019 & .133 & -.009 & $.160\left(^{*}\right)$ & 1.000 & $.682(* *)$ & $.634(* *)$ & $.139\left(^{*}\right)$ \\
\hline & Sig. (2-tailed) & .779 & .051 & .900 & .019 & $\cdot$ & .000 & .000 & .041 \\
\hline & $\mathrm{N}$ & 215 & 214 & 213 & 214 & 215 & 159 & 215 & 215 \\
\hline \multirow[t]{3}{*}{ Knowledge } & $\begin{array}{l}\text { Correlation } \\
\text { Coefficient }\end{array}$ & -.136 & $.210(* *)$ & $.173(*)$ & .059 & $.682(* *)$ & 1.000 & $.756(* *)$ & .025 \\
\hline & Sig. (2-tailed) & .086 & .008 & .030 & .460 & .000 & & .000 & .755 \\
\hline & $\mathrm{N}$ & 161 & 160 & 159 & 160 & 159 & 161 & 161 & 161 \\
\hline \multirow[t]{3}{*}{ Complex } & $\begin{array}{l}\text { Correlation } \\
\text { Coefficient }\end{array}$ & -.028 & $.142(*)$ & .059 & .071 & $.634\left(^{* *}\right)$ & $.756\left({ }^{* *}\right)$ & 1.000 & .044 \\
\hline & Sig. (2-tailed) & .686 & .036 & .388 & .301 & .000 & .000 & $\cdot$ & .520 \\
\hline & $\mathrm{N}$ & 217 & 216 & 215 & 216 & 215 & 161 & 217 & 217 \\
\hline \multirow[t]{3}{*}{ Standards } & $\begin{array}{l}\text { Correlation } \\
\text { Coefficient }\end{array}$ & $.194(* *)$ & .133 & .011 & $.205(* *)$ & $.139\left(^{*}\right)$ & .025 & .044 & 1.000 \\
\hline & Sig. (2-tailed) & .004 & .051 & .873 & .002 & .041 & .755 & .520 & \\
\hline & $\mathrm{N}$ & 217 & 216 & 215 & 216 & 215 & 161 & 217 & 217 \\
\hline
\end{tabular}

*SPSS output, calculated using Spearman’s Rho. 


\section{Appendix 7: Evaluation Study E-Mail Advertisement}

Subject: Help Improve ---- Productivity - New Search Tool

Team,

A multi-phase research project out of --------- has developed a prototype search system specifically optimized for $\mathrm{W}$----- Services. The system provides a single point of access to content from W3, the WWW, and Notes team databases (IC Repository \& AIM Services \& Support Teamroom). The search engine incorporates a unique feature that utilizes relationships between different work activities and document types to provide targeted results.

As an ------ consultant, this system has the potential to improve your productivity. I encourage you all to participate in the evaluation of the system so that it can be improved to be of most value to you. The research team needs a large number of $\mathrm{W}$----- consultants to participate in a user study. The study will take one hour, and will be conducted remotely via a webconference connection on an individual basis. Participants will search for 4 assigned topics and evaluate the results. After the study, participants will be able to continue to use the search engine for their own purposes.

Contact the project team directly to schedule your session:

Luanne Freund

Julie Waterhouse

freund@fis.utoronto.ca

This project is sponsored by ----, and is being conducted as a research project through the -------If you have any questions or concerns about the study, feel free to contact Luanne directly, or contact the Research Staff Member who is supervising this project, Julie Waterhouse ----------(905-XXX-XXXX). 


\section{Appendix 8: Evaluation Study Instruments}

\section{Form 1 - introduction to experiment}

X-Site Search Evaluation - [intro : Form]

\section{Welcome to X-Site Search for Software Services Prototype System Evaluation}

\section{Sponsored by IBM Centre for Advanced Studies, ISSW and ISSL}

The goal of this project is to help Software Services consultants access information more quickly by designing a search system that can search across information resources and retrieve the "right information for the job".

In this study, you will carry out searches using two versions of a prototype search system so that we can evaluate the success of the new system. The system accesses materials from a range of IBM public and internal websites as well as select internal Notes databases.

\section{Continue}

\section{Form 2 - Informed consent}

圆 File Edit Insert Records window Help

Study Procedures and Consent Form

Procedure:

- during the session you will be asked to do four 10-15 minute searches based on assigned search scenarios;

- during each scenario, you will be asked to save or bookmark any useful or relevant pages that you find;

- at the end of each scenario, you will be asked to respond to a few questions regarding the search as a whole;

- throughout the session, your search steps and responses will be recorded automatically via the server log and the forms.

Please note that:

- this research project is sponsored by IBM, which has approved the use of work time to participate:

- your email address will be stored separately from the rest of the data collected, and will not be passed on or used to contact you;

- your identity will not be revealed in the reporting of the study results;

- IBM project coordinators and team managers will not have access to individual participant results;

- your participation is voluntary and you may withdraw at any time.

If you have any questions about this study, please send your request to lfreund@ca.ibm.com Thank-you for your interest and participation!

If you are ready to participate in this study, please submit your email address below:
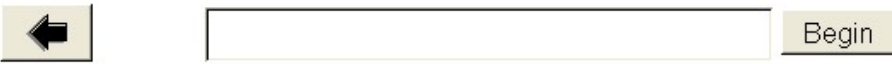


\section{Form 3 - Background information questionnaire}

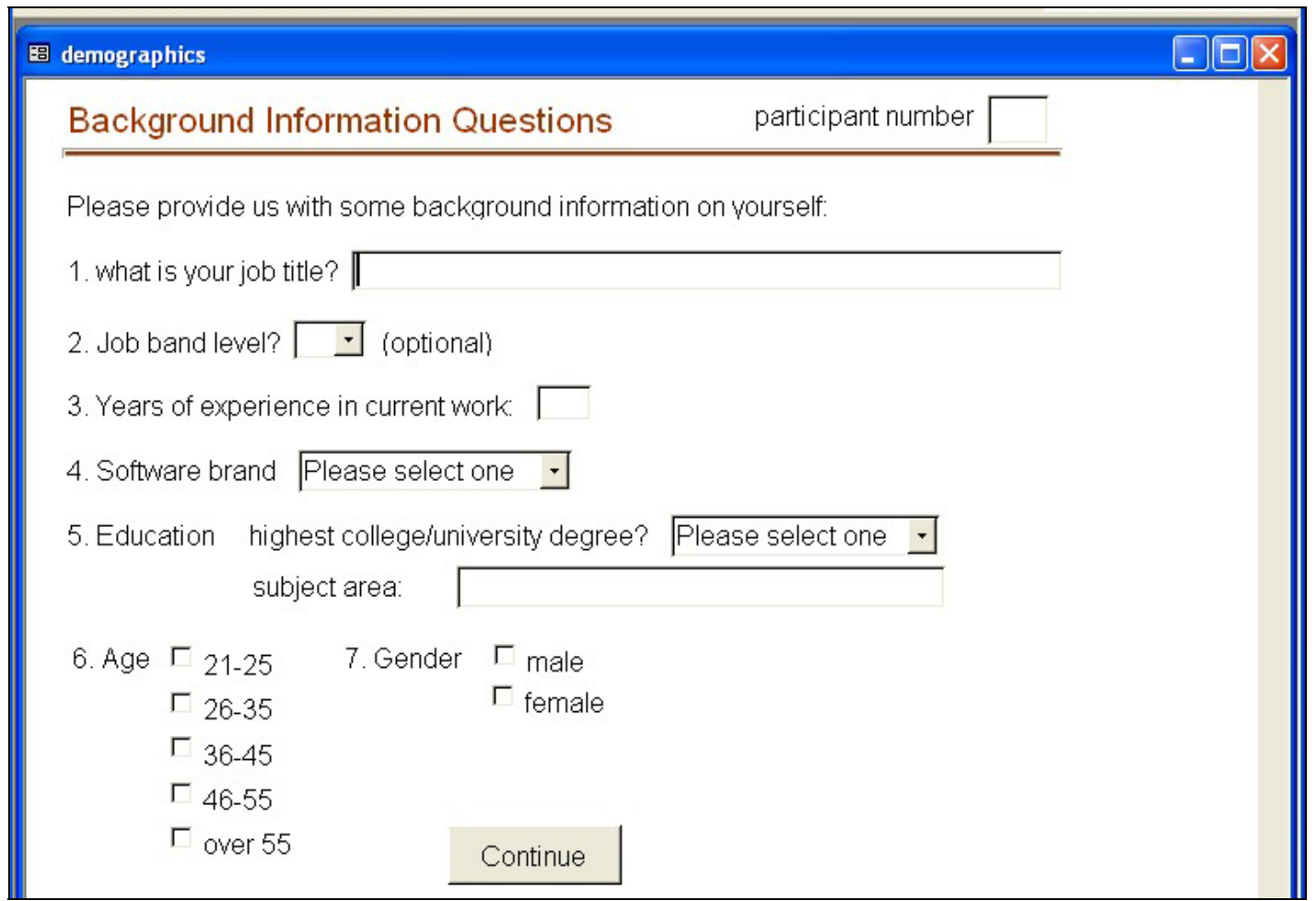

Form 4 - assignment of system and task

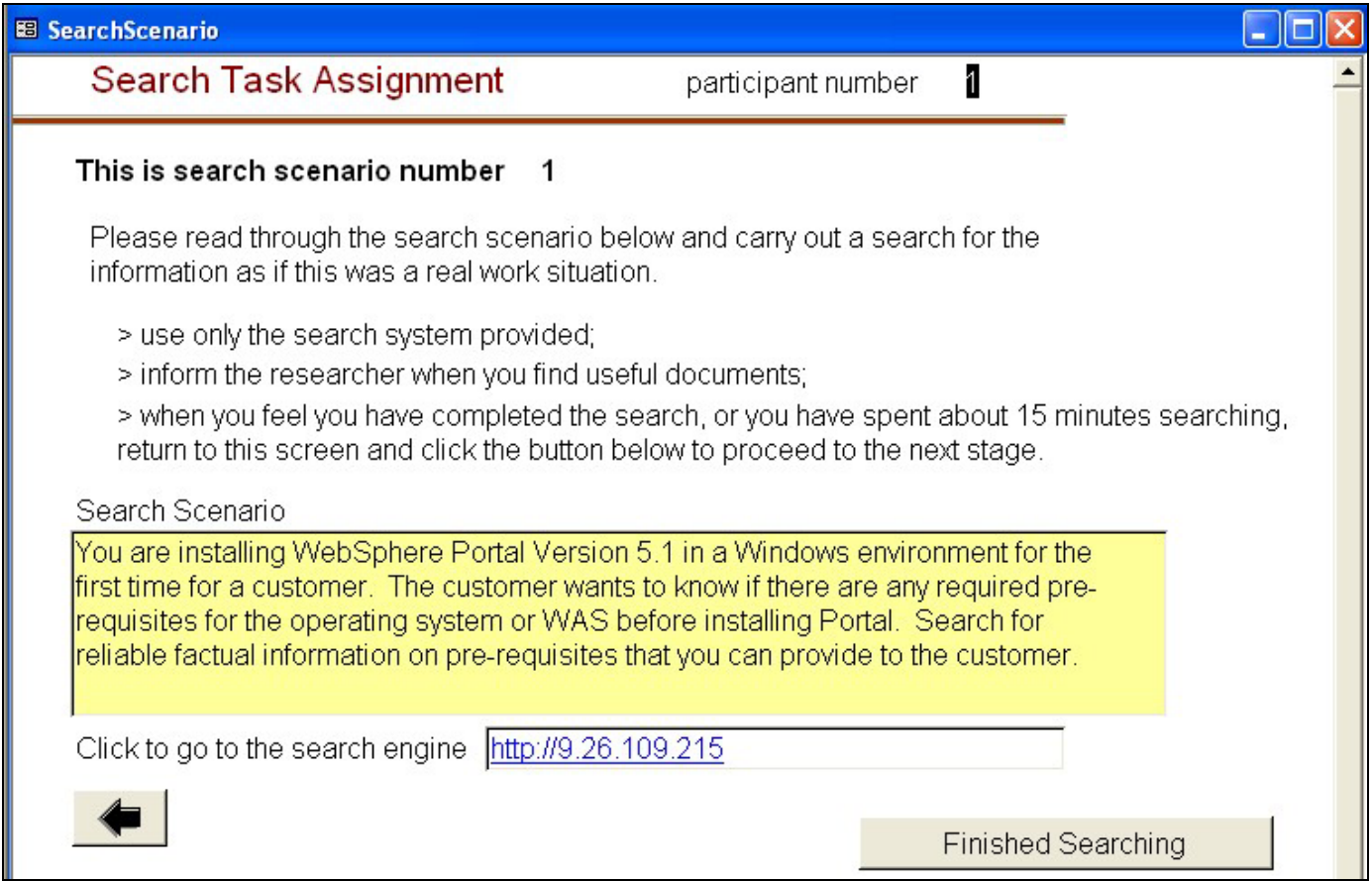




\section{Form 5 - post-task questionnaire}

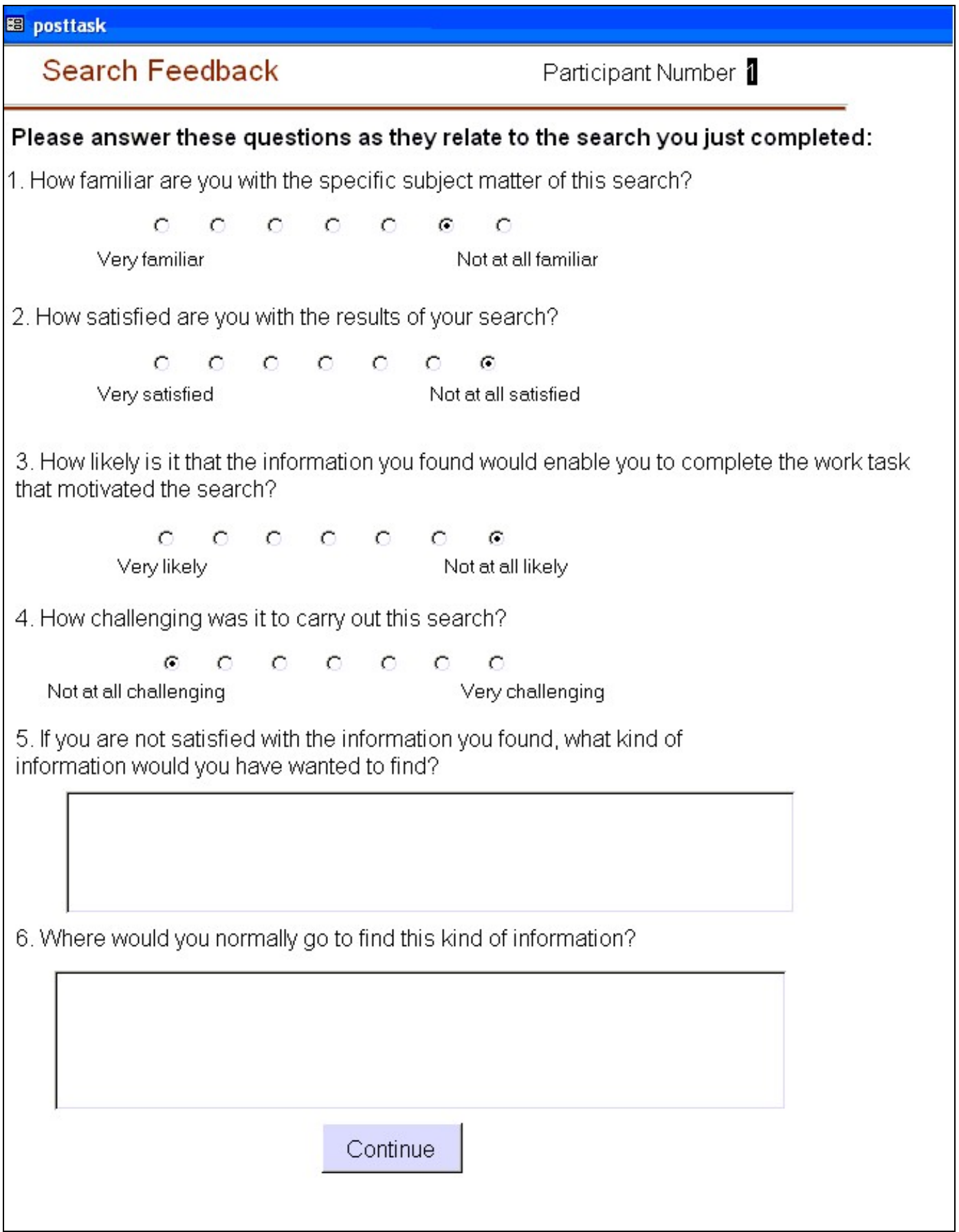




\section{Form 6 - post study feedback form}

\section{Eg feedback}

Final Feedback

Participant Number $\longdiv { 1 }$

Do you have any feedback on the search engine? Things you liked or didn't like? Things you'd like it to be able to do?

Do you have any feedback on the design of the two search interfaces you used?

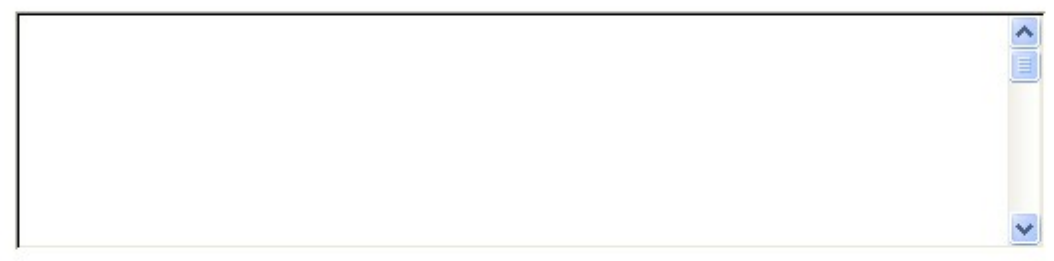

Finished 


\section{Appendix 9: Evaluation Study Log Sheet for Relevant Documents}

Evaluation Study - Relevant Documents Feedback: Participant \#

Page \#

1. Task Num:

Doc Title:

How useful do you think it will be, on a scale of 1-10? barely 122345678910 very

What is it about this document that makes you think it will be useful?

2. Task Num:

Doc Title:

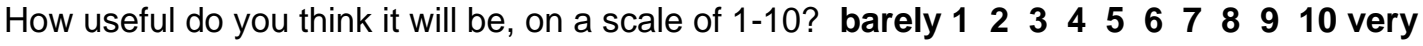
What is it about this document that makes you think it will be useful?

\section{Task Num:}

Doc Title:

How useful do you think it will be, on a scale of 1-10? barely 122345678910 very What is it about this document that makes you think it will be useful?

\footnotetext{
4. Task Num:

Doc Title:

How useful do you think it will be, on a scale of 1-10? barely 122345678910 very What is it about this document that makes you think it will be useful?
}

\section{Task Num:}

Doc Title:

How useful do you think it will be, on a scale of 1-10? barely 124345678910 very What is it about this document that makes you think it will be useful? 


\section{Appendix 10: Evaluation Study Histograms of Dependent Variables}

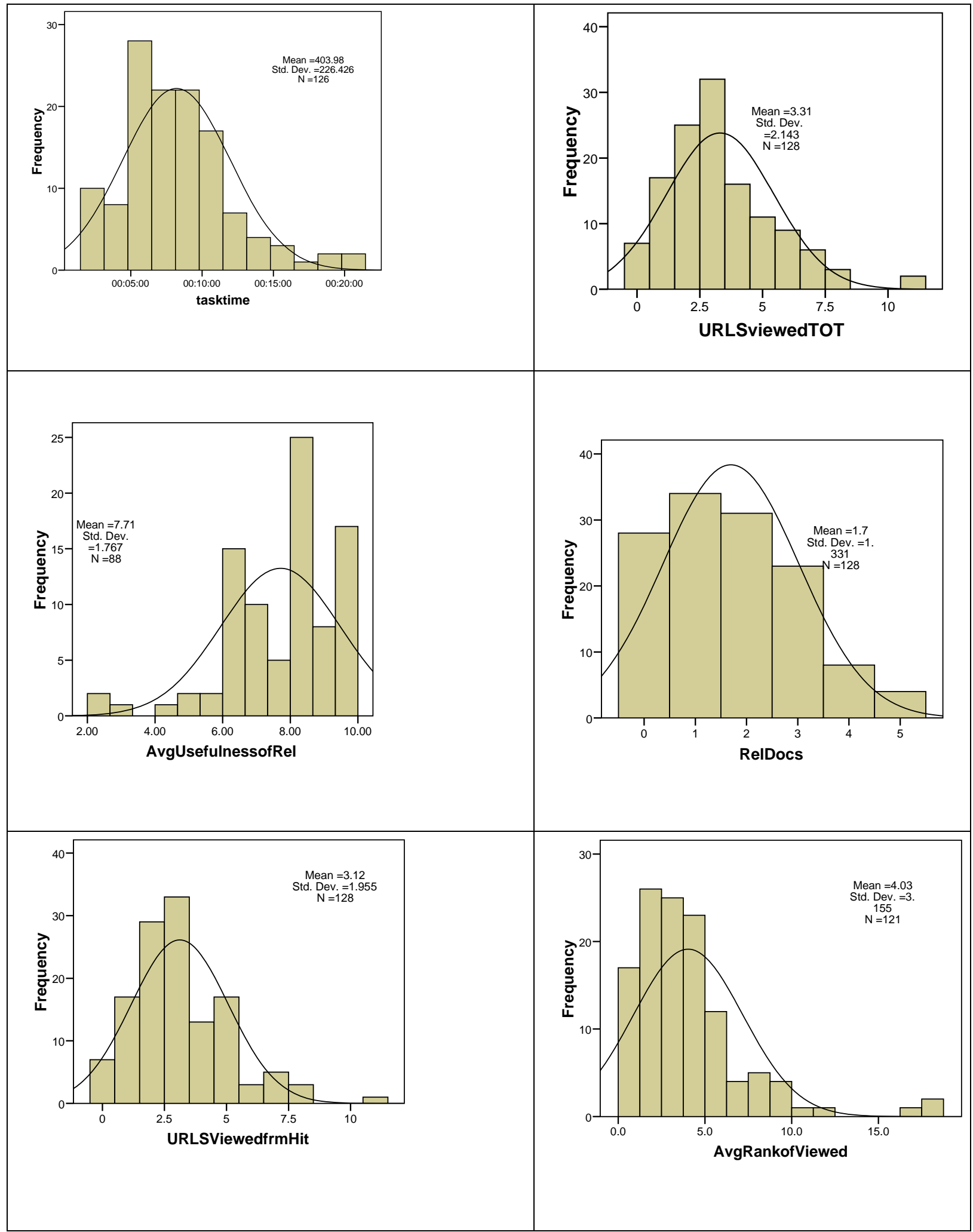




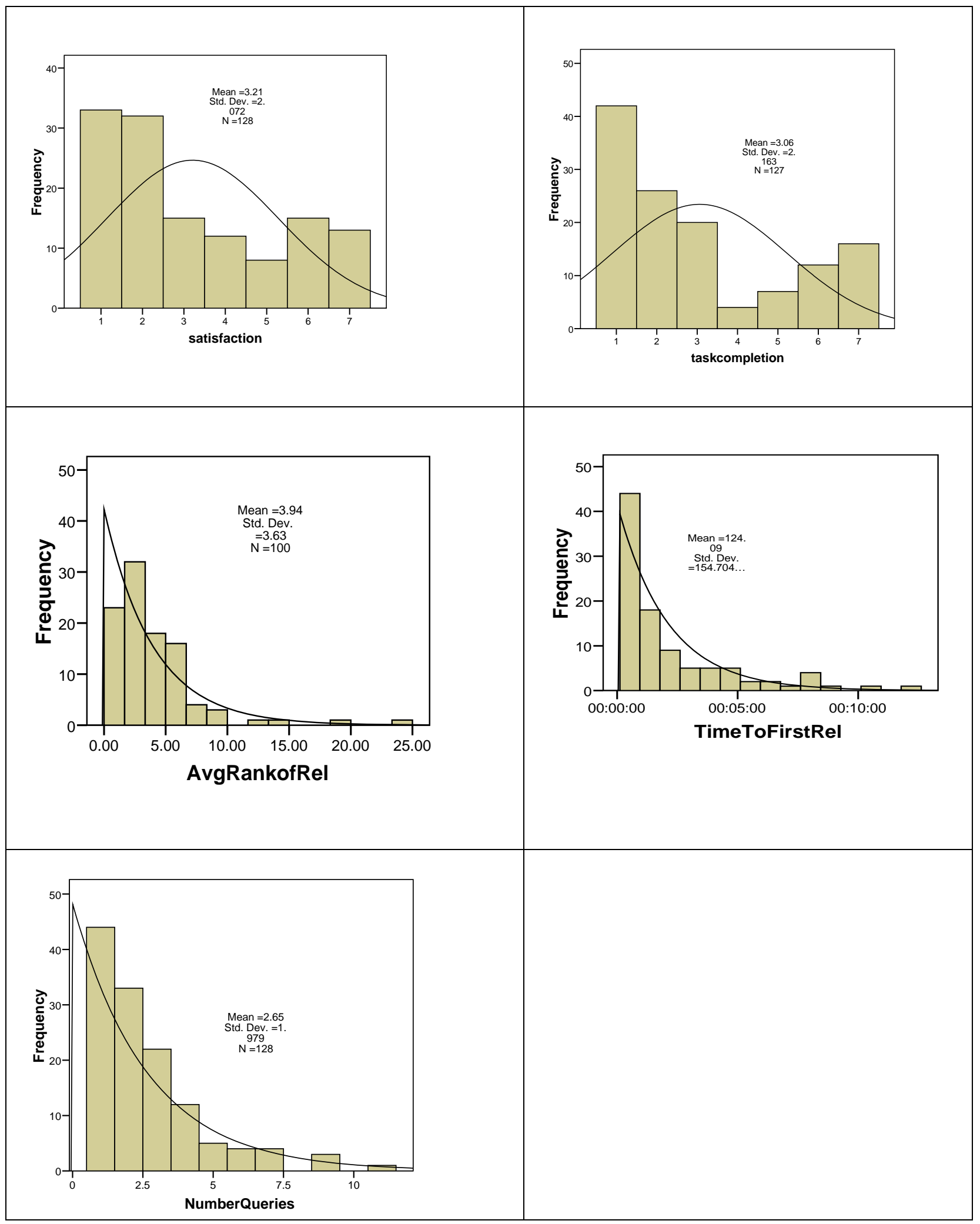

